\title{
THE PERIODIC LORENTZ GAS IN THE BOLTZMANN-GRAD LIMIT: ASYMPTOTIC ESTIMATES
}

\author{
JENS MARKLOF AND ANDREAS STRÖMBERGSSON
}

\begin{abstract}
The dynamics of a point particle in a periodic array of spherical scatterers converges, in the limit of small scatterer size, to a random flight process, whose paths are piecewise linear curves generated by a Markov process with memory two. The corresponding transport equation is distinctly different from the linear Boltzmann equation observed in the case of a random configuration of scatterers. In the present paper we provide asymptotic estimates for the transition probabilities of this Markov process. Our results in particular sharpen previous upper and lower bounds on the distribution of free path lengths obtained by Bourgain, Golse and Wennberg.
\end{abstract}

\section{Contents}

1. Introduction

1.1. The Boltzmann-Grad limit of the periodic Lorentz gas

1.2. Asymptotic estimates for $\xi$ small

1.3. Asymptotic estimates for $\xi$ large

2. Asymptotics of $\Phi_{\mathbf{0}}(\xi, \boldsymbol{w}, \boldsymbol{z})$ for $\xi$ small

2.1. Recollection of definitions

2.2. Proof of Theorem 1.1

2.3. A parametrization of $X_{1}\left(\boldsymbol{e}_{1}, \boldsymbol{y}\right)$ for $d=3$

2.4. Proof of Theorem 1.4

2.5. Explicit formulas for $\Phi(\xi, \boldsymbol{w}), \bar{\Phi}_{\mathbf{0}}(\xi), \Phi_{\mathbf{0}}(\xi)$ and $\Phi(\xi)$ for $d=3, \xi$ small

2.6. Numerical computations for $d=3$

3. Asymptotics for $\Phi(\xi, \boldsymbol{w})$ as $\xi \rightarrow \infty$

3.1. Preliminaries: Iwasawa decomposition and Siegel domains

3.2. A parametrization of lattices with $a_{1}$ large

3.3. An asymptotic formula for $\int_{\xi}^{\infty} \Phi(\eta) d \eta$

3.4. Asymptotics for $\Phi(\xi, \boldsymbol{w})$ : Simplifying the integral

3.5. The paraboloid approximation

3.6. Further simplification of the integral; proof of Theorem 1.11 except for $d=3$

3.7. A slight improvement of the error term for $d=3$

4. The paraboloid approximation

4.1. Definition and basic properties of the general $\Xi$-function

4.2. Some properties of the function $F_{d}(t)$

4.3. On the size and continuity of $\Xi(a, b ; \boldsymbol{h} ; v)$

4.4. Approximating packing probabilites with $\Xi(a, b ; \boldsymbol{h} ; v)$

5. Asymptotics for $\Phi_{\mathbf{0}}(\xi, \boldsymbol{w}, \boldsymbol{z})$ as $\xi \rightarrow \infty$

5.1. Initial reductions

5.2. The main term

5.3. Bounds on some integrals involving $\Xi$

5.4. Proof of Theorem 1.7

6. On the support of $\Phi_{\mathbf{0}}(\xi, \boldsymbol{w}, \boldsymbol{z})$

6.1. The functions $\sigma_{d}(r, \alpha)$ and $\xi_{0}(w, z, \varphi)$

6.2. Bound from below on the support of $\Phi_{\mathbf{0}}$

6.3. An exact formula for $\Phi_{\mathbf{0}}(\xi, \boldsymbol{w}, \boldsymbol{z})$ when $\xi(1-z)^{\frac{d-1}{2}}$ is large

6.4. Bound from above on the support of $\Phi_{0}$

6.5. Proof of Proposition 1.15

7. Asymptotics for $\frac{\partial}{\partial \xi} \Phi(\xi, \boldsymbol{w})$ derived from Theorem 1.7

Index of notations

References

Date: August 29, 2018.

J.M. is supported by a Royal Society Wolfson Research Merit Award. A.S. is a Royal Swedish Academy of Sciences Research Fellow supported by a grant from the Knut and Alice Wallenberg Foundation. 


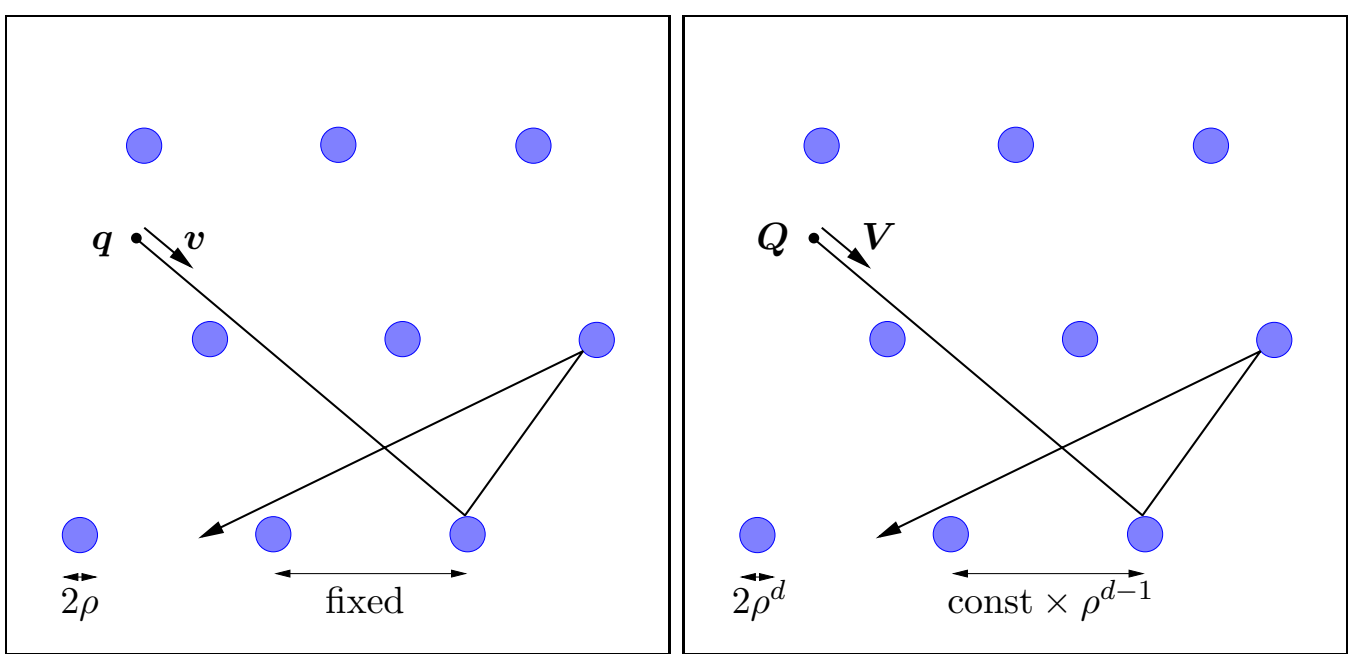

FiguRe 1. Left: The periodic Lorentz gas in "microscopic" coordinates - the lattice $\mathcal{L}$ remains fixed as the radius $\rho$ of the scatterer tends to zero. Right: The periodic Lorentz gas in "macroscopic" coordinates - both the lattice constant and the radius of each scatter tend to zero, in such a way that the mean free path length remains finite.

\section{INTRODUCTION}

The linear Boltzmann equation (also referred to as the Boltzmann-Lorentz equation or kinetic Lorentz equation) is one of the fundamental transport equations that describe the macroscopic dynamics of a dilute gas in matter. The equation was postulated by Lorentz in 1905 [18 by considering a gas of non-interacting point particles moving in an infinite, fixed array of hard sphere scatterers. Crucially, Lorentz assumed that in the limit of small scatterers (Boltzmann-Grad limit) consecutive collisions become independent of each other and are solely determined by the single-scatterer cross section. Lorentz' heuristic derivation of the linear Boltzmann equation was put on a rigorous footing in the case of a random scatterer configuration in the seminal papers by Gallavotti [15, Spohn [27, and Boldrighini, Bunimovich and Sinai [4]. On the other hand, our recent studies of periodic scatterer configurations [20, 21] show that in this case the Boltzmann-Grad limit is governed by a transport equation which is distinctly different from the linear Boltzmann equation. One of the main features is here that (contrary to Lorentz' assumption) consecutive collisions are no longer independent: The collision kernel of our transport equation does not only depend on the particle velocity before and after each collision, but also on the flight time until the next collision and the velocity thereafter. The collision kernel is thus significantly more complicated than in the linear Boltzmann equation, and explicit formulas are so far only known in dimension $d=2$ [22]; cf. also [7], 8] for different approaches. The objective of the present paper is to focus on dimension $d \geq 3$ and derive asymptotic estimates for the collision kernel for small and large inter-collision times. These estimates yield in particular precise asymptotics for the distribution of the free path length in the periodic Lorentz gas, and thus improve the upper and lower bounds obtained by Bourgain, Golse and Wennberg [6], [16].

1.1. The Boltzmann-Grad limit of the periodic Lorentz gas. To explain the setting of our results in more detail, let us fix a euclidean lattice $\mathcal{L} \subset \mathbb{R}^{d}$, and assume (without loss of generality) that its fundamental cell has volume one. We denote by $\mathcal{K}_{\rho} \subset \mathbb{R}^{d}$ the complement of the set $\mathcal{B}_{\rho}^{d}+\mathcal{L}$ (the "billiard domain"), and $\mathrm{T}^{1}\left(\mathcal{K}_{\rho}\right)=\mathcal{K}_{\rho} \times \mathrm{S}_{1}^{d-1}$ its unit tangent bundle (the "phase space"), with $\boldsymbol{q}(t) \in \mathcal{K}_{\rho}$ the position and $\boldsymbol{v}(t) \in \mathrm{S}_{1}^{d-1}$ the velocity of the particle at time $t$. Here $\mathcal{B}_{\rho}^{d}$ denotes the open ball of radius $\rho$, centered at the origin, and $\mathrm{S}_{1}^{d-1}$ the unit sphere. The dynamics of a particle in the Lorentz gas is defined as the motion with unit speed along 
straight lines, and specular reflection at the balls $\mathcal{B}_{\rho}^{d}+\mathcal{L}$. We may in fact also permit other scattering processes, such as the scattering map of a Muffin-tin Coulomb potential; cf. [21] for details. A dimensional argument shows that in the Boltzmann-Grad limit $\rho \rightarrow 0$ the free path length scales like $\rho^{-(d-1)}$, i.e., the inverse of the total scattering cross section of an individual scatterer. It is therefore natural to rescale space and time by introducing the macroscopic coordinates (see Figure 1)

$$
(\boldsymbol{Q}(t), \boldsymbol{V}(t))=\left(\rho^{d-1} \boldsymbol{q}\left(\rho^{-(d-1)} t\right), \boldsymbol{v}\left(\rho^{-(d-1)} t\right)\right) .
$$

The time evolution of a particle with initial data $(\boldsymbol{Q}, \boldsymbol{V})$ is then described by the billiard flow

$$
(\boldsymbol{Q}(t), \boldsymbol{V}(t))=F_{t, \rho}(\boldsymbol{Q}, \boldsymbol{V}) .
$$

Since the speed of our particle is a constant of motion we may assume without loss of generality that $\|\boldsymbol{V}\|=1$. For notational reasons it is convenient to extend the dynamics to the inside of each scatterer trivially, i.e., set $F_{t, \rho}=$ id whenever $\boldsymbol{Q}$ is inside the scatterer. That is, the relevant phase space is now the unit tangent bundle of $\mathbb{R}^{d}$, which will be denoted by $\mathrm{T}^{1}\left(\mathbb{R}^{d}\right)$.

Let us fix a probability measure $\Lambda$ on $\mathrm{T}^{1}\left(\mathbb{R}^{d}\right)$. For random initial data $\left(\boldsymbol{Q}_{0}, \boldsymbol{V}_{0}\right)$ with respect to $\Lambda$, we can then view the billiard flow $\left\{F_{t, \rho}: t>0\right\}$ as a stochastic process. The central result of [20], 21] is that, if $\Lambda$ is absolutely continuous with respect to Lebesque measure, the billiard flow converges in the Boltzmann-Grad limit to a random flight process $\{\Xi(t): t>0\}$, which is defined as the flow with unit speed along a random piecewise linear curve, whose path segments $\boldsymbol{S}_{1}, \boldsymbol{S}_{2}, \boldsymbol{S}_{2}, \ldots \in \mathbb{R}^{d}$ are generated by a Markov process with memory two. Specifically, if we set $\xi_{j}=\left\|\boldsymbol{S}_{j}\right\|$ and $\boldsymbol{V}_{j-1}=\frac{\boldsymbol{S}_{j}}{\left\|\boldsymbol{S}_{j}\right\|}$ for $j=1,2,3, \ldots$, then the distribution of the first $n$ path segments is given by the probability density

$$
\begin{aligned}
& \Lambda^{\prime}\left(\boldsymbol{Q}_{0}, \boldsymbol{V}_{0}\right) p\left(\boldsymbol{V}_{0}, \xi_{1}, \boldsymbol{V}_{1}\right) p_{\mathbf{0}}\left(\boldsymbol{V}_{0}, \boldsymbol{V}_{1}, \xi_{2}, \boldsymbol{V}_{2}\right) \cdots \\
& \quad \cdots p_{\mathbf{0}}\left(\boldsymbol{V}_{n-3}, \boldsymbol{V}_{n-2}, \xi_{n-1}, \boldsymbol{V}_{n-1}\right) \int_{\mathrm{S}_{1}^{d-1}} p_{\mathbf{0}}\left(\boldsymbol{V}_{n-2}, \boldsymbol{V}_{n-1}, \xi_{n}, \boldsymbol{V}_{n}\right) d \mathrm{vol}_{\mathrm{S}_{1}^{d-1}}\left(\boldsymbol{V}_{n}\right),
\end{aligned}
$$

see Theorem 1.3 and Section 4 in [21].

Before describing the transition probability densities $p$ and $p_{\mathbf{0}}$ in more detail, let us explain the relation of our limiting stochastic process $\Xi(t)$ with the macroscopic dynamics of a particle cloud discussed earlier. The time evolution of an initial particle density $f_{0} \in \mathrm{L}^{1}\left(\mathrm{~T}^{1}\left(\mathbb{R}^{d}\right)\right.$ ) in the Lorentz gas with fixed scatterer radius $\rho$ is given by $f_{t}=L_{\rho}^{t} f_{0}$, where $L_{\rho}^{t}$ is the Liouville operator defined by

$$
\left[L_{\rho}^{t} f_{0}\right](\boldsymbol{Q}, \boldsymbol{V}):=f_{0}\left(F_{-t, \rho}(\boldsymbol{Q}, \boldsymbol{V})\right) .
$$

The existence of the limiting stochastic process $\Xi(t)$ implies that for every $t>0$ there exists a linear operator $L^{t}: \mathrm{L}^{1}\left(\mathrm{~T}^{1}\left(\mathbb{R}^{d}\right)\right) \rightarrow \mathrm{L}^{1}\left(\mathrm{~T}^{1}\left(\mathbb{R}^{d}\right)\right)$, such that for every $f_{0} \in \mathrm{L}^{1}\left(\mathrm{~T}^{1}\left(\mathbb{R}^{d}\right)\right)$ and any set $\mathcal{A} \subset \mathrm{T}^{1}\left(\mathbb{R}^{d}\right)$ with boundary of Lebesgue measure zero,

$$
\lim _{\rho \rightarrow 0} \int_{\mathcal{A}}\left[L_{\rho}^{t} f_{0}\right](\boldsymbol{Q}, \boldsymbol{V}) d \boldsymbol{Q} d \mathrm{vol}_{\mathrm{S}_{1}^{d-1}}(\boldsymbol{V})=\int_{\mathcal{A}}\left[L^{t} f_{0}\right](\boldsymbol{Q}, \boldsymbol{V}) d \boldsymbol{Q} d \mathrm{vol}_{\mathrm{S}_{1}^{d-1}}(\boldsymbol{V}) .
$$

If $f_{0}$ is $\mathrm{C}^{1}$ then its image under the limit operator $L^{t}$ is given by

$$
\left[L^{t} f_{0}\right](\boldsymbol{Q}, \boldsymbol{V})=\int_{\mathbb{R}_{>0} \times \mathrm{S}_{1}^{d-1}} f\left(t, \boldsymbol{Q}, \boldsymbol{V}, \xi, \boldsymbol{V}_{+}\right) d \xi d \mathrm{vol}_{\mathrm{S}_{1}^{d-1}}\left(\boldsymbol{V}_{+}\right),
$$

where $f$ is the unique solution of the differential equation

$$
\begin{aligned}
{\left[\partial_{t}+\boldsymbol{V} \cdot \nabla_{\boldsymbol{Q}}-\partial_{\xi}\right] f(t, \boldsymbol{Q}, \boldsymbol{V}} & \left., \xi, \boldsymbol{V}_{+}\right) \\
& =\int_{\mathrm{S}_{1}^{d-1}} f\left(t, \boldsymbol{Q}, \boldsymbol{V}_{0}, 0, \boldsymbol{V}\right) p_{\mathbf{0}}\left(\boldsymbol{V}_{0}, \boldsymbol{V}, \xi, \boldsymbol{V}_{+}\right) d \operatorname{vol}_{\mathrm{S}_{1}^{d-1}}\left(\boldsymbol{V}_{0}\right)
\end{aligned}
$$

subject to the initial condition $f\left(0, \boldsymbol{Q}, \boldsymbol{V}, \xi, \boldsymbol{V}_{+}\right)=f_{0}(\boldsymbol{Q}, \boldsymbol{V}) p\left(\boldsymbol{V}, \xi, \boldsymbol{V}_{+}\right)$. Equation (1.7) corresponds to the Fokker-Planck-Kolmogorov equation of our limiting stochastic process $\Xi(t)$, 


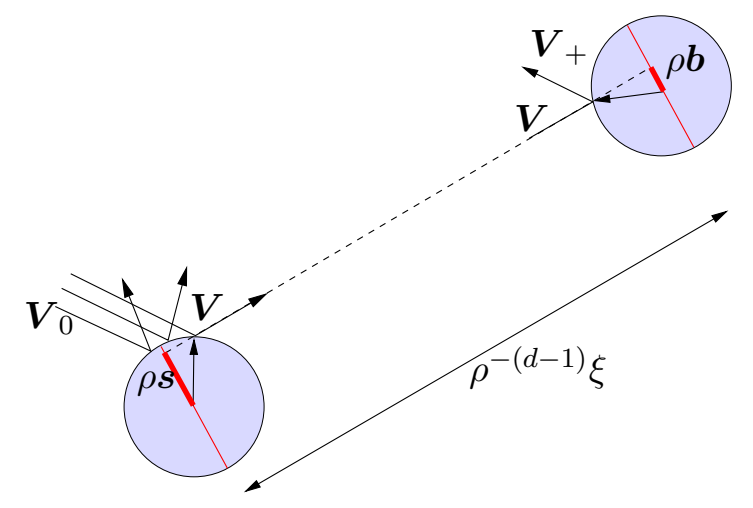

Figure 2. Two consecutive collisions in the Lorentz gas.

cf. Section 6.3 of [21], and may be viewed as a generalization of the linear Boltzmann equation, cf. [19, Section 3].

We can express the probability densities $p\left(\boldsymbol{V}, \xi, \boldsymbol{V}_{+}\right)$and $p_{\mathbf{0}}\left(\boldsymbol{V}_{0}, \boldsymbol{V}, \xi, \boldsymbol{V}_{+}\right)$as

$$
\begin{gathered}
p\left(\boldsymbol{V}, \xi, \boldsymbol{V}_{+}\right)=\sigma\left(\boldsymbol{V}, \boldsymbol{V}_{+}\right) \Phi\left(\xi, \boldsymbol{b}\left(\boldsymbol{V}, \boldsymbol{V}_{+}\right)\right), \\
p_{\mathbf{0}}\left(\boldsymbol{V}_{0}, \boldsymbol{V}, \xi, \boldsymbol{V}_{+}\right)=\sigma\left(\boldsymbol{V}, \boldsymbol{V}_{+}\right) \Phi_{\mathbf{0}}\left(\xi, \boldsymbol{b}\left(\boldsymbol{V}, \boldsymbol{V}_{+}\right),-\boldsymbol{s}\left(\boldsymbol{V}, \boldsymbol{V}_{0}\right)\right)
\end{gathered}
$$

where $\sigma\left(\boldsymbol{V}, \boldsymbol{V}_{+}\right)$is the differential cross section, $\Phi\left(\xi, \boldsymbol{b}\left(\boldsymbol{V}, \boldsymbol{V}_{+}\right)\right)$is the limiting probability density (as $\rho \rightarrow 0$ ) of hitting, from a generic point in $\mathrm{T}^{1}\left(\mathbb{R}^{d}\right)$, the first scatterer at time $\rho^{-(d-1)} \xi$ (in microscopic units) with impact parameter $\boldsymbol{b}\left(\boldsymbol{V}, \boldsymbol{V}_{+}\right)$, and $\Phi_{\mathbf{0}}\left(\xi, \boldsymbol{b}\left(\boldsymbol{V}, \boldsymbol{V}_{+}\right),-\boldsymbol{s}\left(\boldsymbol{V}, \boldsymbol{V}_{0}\right)\right)$ is the limiting probability density of hitting, from a given scatterer with exit parameter $\boldsymbol{s}\left(\boldsymbol{V}, \boldsymbol{V}_{0}\right)$, the next scatterer at time $\rho^{-(d-1)} \xi$ with impact parameter $\boldsymbol{b}\left(\boldsymbol{V}, \boldsymbol{V}_{+}\right)$(cf. Figure 2).

Remark 1.1. If the scattering map is given by specular reflection (as in the original Lorentz gas), we have explicitly $\sigma\left(\boldsymbol{V}, \boldsymbol{V}_{+}\right)=\frac{1}{4}\left\|\boldsymbol{V}-\boldsymbol{V}_{+}\right\|^{3-d}$ for the scattering cross section, and

$$
\boldsymbol{s}\left(\boldsymbol{V}, \boldsymbol{V}_{0}\right)=-\frac{\left(\boldsymbol{V}_{0} K(\boldsymbol{V})\right)_{\perp}}{\left\|\boldsymbol{V}_{0} K(\boldsymbol{V})-\boldsymbol{e}_{1}\right\|}, \quad \boldsymbol{b}\left(\boldsymbol{V}, \boldsymbol{V}_{+}\right)=\frac{\left(\boldsymbol{V}_{+} K(\boldsymbol{V})\right)_{\perp}}{\left\|\boldsymbol{V}_{+} K(\boldsymbol{V})-\boldsymbol{e}_{1}\right\|},
$$

for the exit and impact parameters. Here $\boldsymbol{x}_{\perp}$ denotes the orthogonal projection of $\boldsymbol{x} \in \mathbb{R}^{d}$ onto $\boldsymbol{e}_{1}^{\perp}=\{0\} \times \mathbb{R}^{d-1}$, and for each $\boldsymbol{V} \in \mathrm{S}_{1}^{d-1}$ we have fixed a rotation $K(\boldsymbol{V}) \in \mathrm{SO}(d)$ with $\boldsymbol{V} K(\boldsymbol{V})=\boldsymbol{e}_{1}$.

The study of the asymptotic properties of the probability densities $\Phi(\xi, \boldsymbol{w})$ and $\Phi_{\mathbf{0}}(\xi, \boldsymbol{w}, \boldsymbol{z})$ for $\xi \rightarrow \infty$ and $\xi \rightarrow 0$ are the core objectives of the present paper. Precise formulas for $\Phi$ and $\Phi_{\mathbf{0}}$ in terms of natural probability measures on the homogeneous space $\mathrm{SL}(d, \mathbb{Z}) \backslash \mathrm{SL}(d, \mathbb{R})$ are given in Section 2.1 below. At this point we list the following useful facts:

(A) If $d \geq 3$, the functions

$$
\Phi: \mathbb{R}_{>0} \times \mathcal{B}_{1}^{d-1} \rightarrow[0,1], \quad \Phi_{\mathbf{0}}: \mathbb{R}_{>0} \times \mathcal{B}_{1}^{d-1} \times \mathcal{B}_{1}^{d-1} \rightarrow[0,1]
$$

are continuous.

(B) $\Phi(\xi, \boldsymbol{w})$ depends only on $\xi$ and $\|\boldsymbol{w}\|$; we set

$$
\Phi(\xi, w):=\Phi(\xi, \boldsymbol{w})
$$

with $w=\|\boldsymbol{w}\| \in[0,1)$.

(C) $\Phi_{\mathbf{0}}(\xi, \boldsymbol{w}, \boldsymbol{z})$ depends only on $\xi,\|\boldsymbol{w}\|,\|\boldsymbol{z}\|, \varphi(\boldsymbol{w}, \boldsymbol{z})$ the angle between $\boldsymbol{w}, \boldsymbol{z}$; we set

$$
\Phi_{\mathbf{0}}(\xi, w, z, \varphi):=\Phi_{\mathbf{0}}(\xi, \boldsymbol{w}, \boldsymbol{z})
$$

with $w=\|\boldsymbol{w}\|, z=\|\boldsymbol{z}\| \in[0,1)$ and $\varphi=\varphi(\boldsymbol{w}, \boldsymbol{z}) \in[0, \pi]$. 
(D) $\Phi_{\mathbf{0}}(\xi, \boldsymbol{w}, \boldsymbol{z})=\Phi_{\mathbf{0}}(\xi, \boldsymbol{z}, \boldsymbol{w})$ and thus $\Phi_{\mathbf{0}}(\xi, w, z, \varphi)=\Phi_{\mathbf{0}}(\xi, z, w, \varphi)$.

(E) We have the formulas

$$
\Phi(\xi, \boldsymbol{w})=\int_{\xi}^{\infty} \int_{\mathcal{B}_{1}^{d-1}} \Phi_{\mathbf{0}}(\eta, \boldsymbol{w}, \boldsymbol{z}) d \boldsymbol{z} d \eta, \quad \lim _{\xi \rightarrow 0} \Phi(\xi, \boldsymbol{w})=1 .
$$

(A)-(D) follow from [20, Remark 4.5] and are proved in Sections 8.1 and 8.2 of that paper. (E) follows from [21, Remark 6.2] and [20, (4.16)].

Remark 1.2. In dimension $d=2$ we have the following explicit formula for the transition probability [22]:

$$
\Phi_{\mathbf{0}}(\xi, \boldsymbol{w}, \boldsymbol{z})=\frac{6}{\pi^{2}} \Upsilon\left(1+\frac{\xi^{-1}-\max (|\boldsymbol{w}|,|\boldsymbol{z}|)-1}{|\boldsymbol{w}+\boldsymbol{z}|}\right)
$$

with

$$
\Upsilon(x)= \begin{cases}0 & \text { if } x \leq 0 \\ x & \text { if } 0<x<1 \\ 1 & \text { if } 1 \leq x,\end{cases}
$$

The same formula has recently been found independently by Caglioti and Golse [8] and by Bykovskii and Ustinov [7], using different methods based on continued fractions.

Remark 1.3. All of the relations stated in (A)-(E) above are also valid for the Lorentz gas with random scatterer configuration. Here the fundamental function $\Phi_{\mathbf{0}}$ is given by the explicit formula

$$
\Phi_{\mathbf{0}}(\xi, \boldsymbol{w}, \boldsymbol{z})=\exp \left(-v_{d-1} \xi\right),
$$

where $v_{d-1}=\pi^{\frac{d-1}{2}} \Gamma\left(\frac{d+1}{2}\right)^{-1}$ denotes (throughout this paper) the volume of the unit ball in $\mathbb{R}^{d-1}$. Note that relation (E) implies with (1.17) that in the random setting $\Phi(\xi, \boldsymbol{w})=$ $\Phi_{\mathbf{0}}(\xi, \boldsymbol{w}, \boldsymbol{z})$.

1.2. Asymptotic estimates for $\xi$ small. Returning to the setting of the periodic Lorentz gas, we first state the asymptotic formulas for $\Phi$ and $\Phi_{0}$ as $\xi \rightarrow 0$. Our main result in this direction is the following.

Theorem 1.1. For $\xi>0$ and $\boldsymbol{w}, \boldsymbol{z} \in \mathcal{B}_{1}^{d-1}$,

$$
\frac{1-2^{d-1} v_{d-1} \xi}{\zeta(d)} \leq \Phi_{\mathbf{0}}(\xi, \boldsymbol{w}, \boldsymbol{z}) \leq \frac{1}{\zeta(d)} .
$$

That is, $\Phi_{\mathbf{0}}(\xi, \boldsymbol{w}, \boldsymbol{z})=\zeta(d)^{-1}+O(\xi)$, where the remainder term is everywhere non-positive, and the implied constant is independent of $\boldsymbol{w}$ and $\boldsymbol{z}$. Note that this estimate is consistent with formula (1.15) in dimension $d=2$, where we have the exact relation $\Phi_{\mathbf{0}}(\xi, \boldsymbol{w}, \boldsymbol{z})=\frac{6}{\pi^{2}}=\zeta(2)^{-1}$ for $\xi \leq \frac{1}{2}$.

Remark 1.4. In the case of a random scatterer configuration, (1.17) yields $\Phi_{\mathbf{0}}(\xi, \boldsymbol{w}, \boldsymbol{z})=1+$ $O(\xi)$. Comparing this with Theorem 1.1, we may conclude that the leading-order asymptotics of the transition probability density $\Phi_{\mathbf{0}}(\xi, \boldsymbol{w}, \boldsymbol{z})$ for $\xi \rightarrow 0$ is, in both the random and periodic set-up, independent of $\boldsymbol{w}, \boldsymbol{z}$, and given by the relative density of scatterers which are completely visible from a given scatterer: in the random setting, this is the case for all scatterers close to the given scatterer; in the periodic setting the same holds only for scatterers located on visible (or primitive) lattice points, whose relative density is given by $\zeta(d)^{-1}$.

Theorem 1.1 combined with (1.14) immediately implies:

Corollary 1.2. For all $\xi>0$ and $\boldsymbol{w} \in \mathcal{B}_{1}^{d-1}$,

$$
\Phi(\xi, \boldsymbol{w})=1-\frac{v_{d-1}}{\zeta(d)} \xi+O\left(\xi^{2}\right),
$$

where the remainder term is everywhere non-negative, and the implied constant is independent of $\boldsymbol{w}$. 

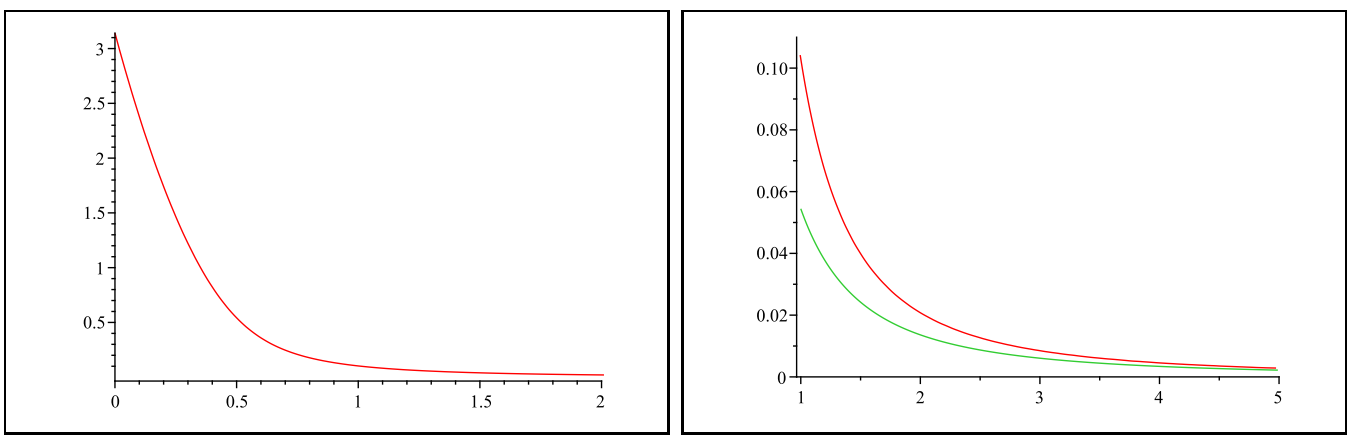

Figure 3. Graphs of $\Phi(\xi)$ for $d=3$ in the ranges $0<\xi \leq 2$ and $1 \leq \xi \leq 5$, obtained from numerical computations described in Section 2.6. In the second plot also the asymptotic $\xi \mapsto \frac{\pi}{48 \zeta(3)} \xi^{-2}$ from Theorem 1.14 is shown (the lower curve).

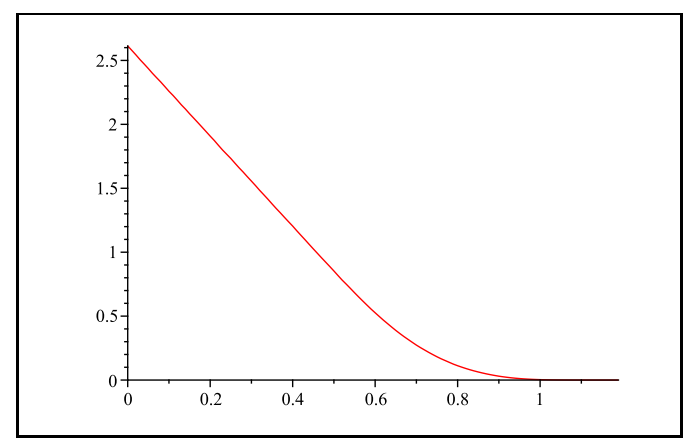

Figure 4. Graph of $\Phi_{0}(\xi)$ for $d=3$, obtained from numerical computations described in Section 2.6 .

From our asymptotics for $\Phi(\xi, \boldsymbol{w})$ and $\Phi_{\mathbf{0}}(\xi, \boldsymbol{w}, \boldsymbol{z})$ we can also derive asymptotics for the limiting distribution of the free path length. We have (cf. [20, Remark 4.6])

$$
\bar{\Phi}_{\mathbf{0}}(\xi)=\frac{1}{v_{d-1}} \int_{\mathcal{B}_{1}^{d-1}} \int_{\mathcal{B}_{1}^{d-1}} \Phi_{\mathbf{0}}(\xi, \boldsymbol{w}, \boldsymbol{z}) d \boldsymbol{w} d \boldsymbol{z}
$$

for the free path length between consecutive collisions,

$$
\Phi(\xi)=\int_{\mathcal{B}_{1}^{d-1}} \Phi(\xi, \boldsymbol{w}) d \boldsymbol{w}=v_{d-1} \int_{\xi}^{\infty} \bar{\Phi}_{\mathbf{0}}(\eta) d \eta
$$

for the free path length from a generic initial point inside the billiard domain, and

$$
\Phi_{\mathbf{0}}(\xi)=\int_{\mathcal{B}_{1}^{d-1}} \Phi_{\mathbf{0}}(\xi, \boldsymbol{w}, \mathbf{0}) d \boldsymbol{w}
$$

for the free path length of a particle starting at a lattice point (with the scatterer removed).

Only in dimension $d=2$ we have explicit formulas for the above limiting distributions, thanks to the work of Dahlqvist [12], Boca, Gologan and Zaharescu [2], and Boca and Zaharescu [3].

Using Theorem 1.1 and Corollary 1.2 in conjunction with (1.20), (1.21), (1.22), we obtain the following.

Corollary 1.3. For $\xi>0$,

$$
\bar{\Phi}_{\mathbf{0}}(\xi)=\frac{v_{d-1}}{\zeta(d)}+O(\xi) ; \quad \Phi_{\mathbf{0}}(\xi)=\frac{v_{d-1}}{\zeta(d)}+O(\xi) ; \quad \Phi(\xi)=v_{d-1}-\frac{v_{d-1}^{2}}{\zeta(d)} \xi+O\left(\xi^{2}\right) .
$$

Here the first two remainder terms are $\leq 0$ and the last remainder term is $\geq 0$, for all $\xi>0$. 
Remark 1.5. Formulas (1.20), (1.21), (1.22) are also valid in the random setting, and yield

$$
\bar{\Phi}_{\mathbf{0}}(\xi)=\Phi_{\mathbf{0}}(\xi)=\Phi(\xi)=v_{d-1} \exp \left(-v_{d-1} \xi\right)=v_{d-1}-v_{d-1}^{2} \xi+O\left(\xi^{2}\right) .
$$

These asymtotics are the same as in the periodic setting (1.23), with the relative density of visible lattice points $\zeta(d)^{-1}$ replaced by 1 .

In the case $d=3$ we are in fact able to compute $\Phi_{\mathbf{0}}(\xi, \boldsymbol{w}, \boldsymbol{z})$ explicitly for $\xi$ small. For $0 \leq t<1$ we set

$$
F(t):=\pi-\arccos (t)+t \sqrt{1-t^{2}}=\operatorname{Area}\left(\left\{\left(x_{1}, x_{2}\right) \in \mathcal{B}_{1}^{2}: x_{1}<t\right\}\right) .
$$

Also let $\xi_{1}: \mathcal{B}_{1}^{2} \times \mathcal{B}_{1}^{2} \rightarrow \mathbb{R}$ be the continuous function given by $\xi_{1}(\boldsymbol{w}, \boldsymbol{z})=(6 \mathrm{~V})^{-1}$, where $V$ is the largest possible volume of a tetrahedron which is contained in the closed cylinder $[0,1] \times \overline{\mathcal{B}_{1}^{2}}$ and which has one vertex at $(0,-\boldsymbol{z})$ and another at $(1, \boldsymbol{w})$.

Theorem 1.4. If $d=3$, then for all $\boldsymbol{w}, \boldsymbol{z} \in \mathcal{B}_{1}^{2}$ and all $0<\xi \leq \xi_{1}(\boldsymbol{w}, \boldsymbol{z})$,

$$
\Phi_{\mathbf{0}}(\xi, \boldsymbol{w}, \boldsymbol{z})=\zeta(3)^{-1}\left(1-\frac{6}{\pi^{2}} F\left(\frac{1}{2}\|\boldsymbol{w}-\boldsymbol{z}\|\right) \xi\right) .
$$

We will prove in Lemma 2.2 below that $\frac{1}{4}<\xi_{1}(\boldsymbol{w}, \boldsymbol{z}) \leq 1$ for all $\boldsymbol{w}, \boldsymbol{z} \in \mathcal{B}_{1}^{2}$, where both the lower and the upper bound are sharp. In particular the formula (1.26) is always true when $0<\xi \leq \frac{1}{4}$.

Combining Theorem 1.4 with (1.14) we will prove the following:

Corollary 1.5. If $d=3$, then for all $\boldsymbol{w} \in \mathcal{B}_{1}^{2}$ and all $0<\xi \leq \min \left(\frac{1}{2(1+\|\boldsymbol{w}\|)}, \frac{2}{3 \sqrt{3}}\right)$ :

$$
\Phi(\xi, \boldsymbol{w})=1-\frac{\pi}{\zeta(3)} \xi+\frac{6}{\pi^{2} \zeta(3)} G(\|\boldsymbol{w}\|) \xi^{2},
$$

where $G:[0,1] \rightarrow \mathbb{R}_{>0}$ is the function

$$
G(w)=\pi \int_{0}^{1-w} F\left(\frac{1}{2} r\right) r d r+\int_{1-w}^{1+w} F\left(\frac{1}{2} r\right) \arccos \left(\frac{w^{2}+r^{2}-1}{2 w r}\right) r d r .
$$

The function $G(w)$ is continuous and strictly increasing, and satisfies $G(0)=\frac{\pi(4 \pi+3 \sqrt{3})}{16}$ and $G(1)=\frac{5}{16} \pi^{2}+1$.

Furthermore, using Theorem 1.4 in conjunction with (1.20), (1.21), (1.22), we will prove:

Corollary 1.6. If $d=3$, then

$$
\begin{array}{ll}
\bar{\Phi}_{\mathbf{0}}(\xi)=\frac{\pi}{\zeta(3)}-\frac{3 \pi^{2}+16}{\pi^{2} \zeta(3)} \xi & \text { for all } 0<\xi \leq \frac{1}{4} \\
\Phi(\xi)=\pi-\frac{\pi^{2}}{\zeta(3)} \xi+\frac{3 \pi^{2}+16}{2 \pi \zeta(3)} \xi^{2} & \text { for all } 0<\xi \leq \frac{1}{4} \\
\Phi_{\mathbf{0}}(\xi)=\frac{\pi}{\zeta(3)}-\frac{3(4 \pi+3 \sqrt{3})}{4 \pi \zeta(3)} \xi & \text { for all } 0<\xi \leq \frac{2}{3 \sqrt{3}}(=0.3849 \ldots) .
\end{array}
$$

Figures 3 and 4 show graphs of $\Phi(\xi)$ and $\Phi_{0}(\xi)$ for $d=3$, obtained by numerical computations described in Section 2.6.

1.3. Asymptotic estimates for $\xi$ large. The case of $\xi \rightarrow \infty$ is much more complicated than $\xi$ small. Among other things, difficulties are caused by the fact that for large $\xi, \Phi_{\mathbf{0}}(\xi, w, z, \varphi)$ vanishes unless both $w$ and $z$ are near 1 .

The following theorem gives an asymptotic formula for $\Phi_{\mathbf{0}}(\xi, w, z, \varphi)$ as $\xi \rightarrow \infty$, for $\varphi$ small. The case of small $\varphi$ is in a natural sense the most important one. Indeed, it was seen in [29] that, for given large $\xi$, the function $\Phi_{\mathbf{0}}(\xi, w, z, \varphi)$ takes its largest values when $\varphi$ is small, and also that it has its largest support with respect to $w, z$ in this case. In particular, when integrating $\Phi_{\mathbf{0}}(\xi, \boldsymbol{w}, \boldsymbol{z})$ over $\boldsymbol{z} \in \mathcal{B}_{1}^{d-1}$, the main contribution comes from $\boldsymbol{z}$ with $\varphi(\boldsymbol{w}, \boldsymbol{z}) \ll \xi^{-\frac{1}{d}+\varepsilon}$. This follows directly from Theorem 1.9 and Proposition 1.10 below (cf. also [29, Cor. 1.9]). 
Theorem 1.7. There exists a continuous and uniformly bounded function $F_{\mathbf{0}, d}: \mathbb{R}_{>0} \times \mathbb{R}_{>0} \times$ $\mathbb{R}_{\geq 0} \rightarrow \mathbb{R}_{\geq 0}$ such that

$$
\Phi_{\mathbf{0}}(\xi, w, z, \varphi)=\xi^{-2+\frac{2}{d}} F_{\mathbf{0}, d}\left(\xi^{\frac{2}{d}}(1-z), \xi^{\frac{2}{d}}(1-w), \xi^{\frac{1}{d}} \varphi\right)+O(E),
$$

for all $\xi>0, w, z \in[0,1), \varphi \in\left[0, \frac{\pi}{2}\right)$, where the error term is

$$
E= \begin{cases}\xi^{-2} & \text { if } d=2, \\ \xi^{-2} \log \left(2+\min \left(\xi, \varphi^{-1}\right)\right) & \text { if } d=3, \\ \min \left(\xi^{-2}, \xi^{-3+\frac{2}{d-1}} \varphi^{2-d+\frac{2}{d-1}}\right) & \text { if } d \geq 4 .\end{cases}
$$

In dimension $d=2$ we have from [22, Eq. (36)] (note that $\varphi=0$ or $=\pi$ in this case)

$$
F_{\mathbf{0}, 2}\left(t_{1}, t_{2}, 0\right)=\frac{3}{\pi^{2}}\left(1-\max \left(t_{1}, t_{2}\right)\right)^{+} .
$$

For $d \geq 3$, we will express $F_{\mathbf{0}, d}$ as an integral of a function given by the probability that a random lattice in $\mathbb{R}^{d-1}$ is disjoint from a union of two cut paraboloids (cf. Section 4.1 and (5.66) below). This integral representation will imply the following properties:

- Symmetry: $F_{\mathbf{0}, d}\left(t_{1}, t_{2}, \alpha\right)=F_{\mathbf{0}, d}\left(t_{2}, t_{1}, \alpha\right)$ (cf. (4.9) and (5.66) $)$.

- Support: There is a continuous function $\sigma_{d}: \mathbb{R}_{>0} \times \mathbb{R}_{>0} \rightarrow \mathbb{R}_{>0}$, which we will define in terms of a lattice problem in dimension $d-1$ (cf. (6.3) below), such that

$$
F_{\mathbf{0}, d}\left(t_{2}, t_{1}, \alpha\right)>0 \Longleftrightarrow t_{1} t_{2}<\sigma_{d}\left(\frac{t_{2}}{t_{1}}, \frac{\alpha^{4}}{t_{1} t_{2}}\right) \text {. }
$$

This function $\sigma_{d}$ satisfies the symmetry relation $\sigma_{d}(r, \alpha)=\sigma_{d}\left(r^{-1}, \alpha\right)$, and we also have $\sigma_{d}(r, \alpha) \asymp r \min \left(1,(r \alpha)^{-\frac{1}{d}}\right)$ uniformly over $r \in(0,1], \alpha \geq 0$ (cf. (6.5) below). It follows that there exist constants $c^{\prime}>c>0$ which only depend on $d$ such that

$$
\begin{aligned}
& \max \left(t_{1}, t_{2}\right) \cdot \max \left(1, \alpha^{\frac{2}{d-1}}\right) \geq c^{\prime} \Longrightarrow F_{\mathbf{0}, d}\left(t_{2}, t_{1}, \alpha\right)=0 ; \\
& \max \left(t_{1}, t_{2}\right) \cdot \max \left(1, \alpha^{\frac{2}{d-1}}\right)<c \Longrightarrow F_{\mathbf{0}, d}\left(t_{2}, t_{1}, \alpha\right)>0 .
\end{aligned}
$$

In particular the support of $F_{\mathbf{0}, d}$ is contained in $\left(0, c^{\prime}\right] \times\left(0, c^{\prime}\right] \times \mathbb{R}_{\geq 0}$, and for any given $t_{1}, t_{2}, F_{\mathbf{0}, d}\left(t_{1}, t_{2}, \cdot\right)$ has compact support in the third variable.

- Bounds from above: $F_{\mathbf{0}, d}$ is uniformly bounded (as mentioned), and we have the following more precise bound (cf. (5.66) and Lemma 5.3 below):

$$
F_{\mathbf{0}, d}\left(t_{1}, t_{2}, \alpha\right) \ll \min \left(1, \alpha^{-d+\frac{2}{d-1}}\right) .
$$

- Bounds from below: For general $d$ it follows from Theorem 1.7 combined with [29, Prop. 7.8] that $F_{\mathbf{0}, d}$ is uniformly bounded from below for $t_{1}, t_{2}, \alpha$ near zero, viz. there is a small constant $c>0$ which only depends on $d$ such that

$$
\max \left(t_{1}, t_{2}, \alpha\right)<c \Longrightarrow F_{\mathbf{0}, d}\left(t_{2}, t_{1}, \alpha\right)>c .
$$

For $d=3$ we have a stronger result (cf. [29, Prop. 7.7]) which says that (1.34) is sharp in a natural sense; but we expect that (1.34) is not sharp for $d \geq 4$.

The lower bound implies that the main term dominates as $\xi \rightarrow \infty$ in (1.29) whenever $1-z<c \xi^{-\frac{2}{d}}, 1-w<c \xi^{-\frac{2}{d}}$ and $\varphi<c \xi^{-\frac{1}{d}}$. Note that this $z, w, \varphi$-regime contributes a positive portion to the integral (1.14). Beyond this regime, our motivation for Theorem 1.7 is that even though we cannot say exactly where the main term dominates, it dominates on a sufficiently large set so that we get a good asymptotics for $\Phi(\xi, w)$ via (1.14), see Theorem 1.11 below.

Next we give a closely related result on the asymptotic shape of the support of $\Phi_{\mathbf{0}}$ for $\xi$ large and $\varphi$ small. Note that by (1.32), the main term in (1.29) is non-zero if and only if

$$
\xi<(1-z)^{-\frac{d}{4}}(1-w)^{-\frac{d}{4}} \sigma_{d}\left(\frac{1-w}{1-z}, \frac{\varphi^{4}}{(1-z)(1-w)}\right)^{\frac{d}{4}} .
$$


However, clearly Theorem 1.7 never helps us deduce $\Phi_{\mathbf{0}}=0$, and furthermore since (as discussed above) it is difficult to state when the main term dominates the error term in Theorem 1.7, we cannot deduce $\Phi_{\mathbf{0}} \neq 0$ in any reasonable region either. By using some intermediate formulas from the proof of Theorem 1.7 (see Section 6 for details) we are, however, able to prove that (1.36) gives in fact a good approximation of the support of $\Phi_{0}$ for $\xi$ large:

Theorem 1.8. There is a continuous function $\xi_{0}:[0,1) \times[0,1) \times[0, \pi] \rightarrow \mathbb{R}_{>0}$ such that $\Phi_{\mathbf{0}}(\xi, w, z, \varphi)>0$ holds if and only if $\xi<\xi_{0}(w, z, \varphi)$. This function $\xi_{0}(w, z, \varphi)$ satisfies

$$
\begin{aligned}
\xi_{0}(w, z, \varphi)=(1-z)^{-\frac{d}{4}}(1-w)^{-\frac{d}{4}} \sigma_{d} & \left(\frac{1-w}{1-z}, \frac{\varphi^{4}}{(1-z)(1-w)}\right)^{\frac{d}{4}} \\
& \times\left\{1+O\left(\max (1-z, 1-w)+\varphi^{2}\right)\right\},
\end{aligned}
$$

uniformly over all $z, w \in[0,1), \varphi \in\left[0, \frac{\pi}{2}\right]$. (The implied constant depends only on d.)

To complement the picture of $\Phi_{\mathbf{0}}$ for $\xi$ large, we recall the uniform bounds on the size and support of $\Phi_{0}$ which the second author proved in [29].

Theorem 1.9. (29, Thm. 1.8]) Let $d \geq 3$. We then have, for all $\xi>0, \boldsymbol{w}, \boldsymbol{z} \in \mathcal{B}_{1}^{d-1}$, and writing $\varphi=\varphi(\boldsymbol{w}, \boldsymbol{z})$,

$$
\Phi_{\mathbf{0}}(\xi, \boldsymbol{w}, \boldsymbol{z}) \ll \begin{cases}\xi^{-2+\frac{2}{d}} \min \left\{1,\left(\xi \varphi^{d}\right)^{-1+\frac{2}{d(d-1)}}\right\} & \text { if } \varphi \leq \frac{\pi}{2} \\ \xi^{-2} \min \left\{1,\left(\xi(\pi-\varphi)^{d-2}\right)^{-1+\frac{2}{d-1}}\right\} & \text { if } \varphi \geq \frac{\pi}{2}\end{cases}
$$

Proposition 1.10. ([29, Prop. 1.9]) Let $d \geq 3$. We then have for all $z, w \in[0,1), \varphi \in[0, \pi]$, writing $t:=\max (1-z, 1-w)$,

$$
\xi_{0}(w, z, \varphi) \asymp \begin{cases}\min \left(t^{-\frac{d}{2}}, t^{-\frac{d-1}{2}} / \varphi\right) & \text { if } \varphi \leq \frac{\pi}{2} \\ \max \left(t^{-\frac{d-2}{2}}, t^{-\frac{d-1}{2}}(\pi-\varphi)\right) & \text { if } \varphi \geq \frac{\pi}{2}\end{cases}
$$

where the implied constants only depend on $d$. (If $\varphi=0$ then the right hand side of (1.39) should be interpreted as $t^{-\frac{d}{2}}$.)

Note that the case $\varphi \leq \frac{\pi}{2}$ of Theorem [1.9 can be recovered from Theorem 1.7 and (1.34); and the case $\varphi \leq \frac{\pi}{2}$ of Proposition 1.10 can be recovered from Theorem 1.8 combined with the fact that $\sigma_{d}(r, \alpha) \asymp r \min \left(1,(r \alpha)^{-\frac{1}{d}}\right)$ uniformly over $r \in(0,1], \alpha \geq 0$. In [29] it was also proved that the upper bound in Theorem 1.8 is sharp in a natural sense for $d=3$, and also for general $d \geq 3$ if either $\varphi \ll \xi^{-\frac{1}{d}}$ or $\pi-\varphi \ll \xi^{-\frac{1}{d-2}}$.

Next we give an asymptotic formula for $\Phi(\xi, w)$ for $\xi$ large.

Theorem 1.11. There exists a bounded continuous function $F_{d}: \mathbb{R}_{>0} \rightarrow \mathbb{R}_{\geq 0}$ such that

$$
\Phi(\xi, w)=\xi^{-2+\frac{2}{d}} F_{d}\left(\xi^{\frac{2}{d}}(1-w)\right)+O(1)\left\{\begin{array}{ll}
\xi^{-2} & \text { if } d=2 \\
\xi^{-2} \log \left(2+\min \left(\xi, \xi^{-\frac{2}{3}}(1-w)^{-1}\right)\right) & \text { if } d=3 \\
\xi^{-2} & \text { if } d \geq 4
\end{array}\right\},
$$

as $\xi \rightarrow \infty$, uniformly over all $0 \leq w<1$.

For $d=2$, we have explicitly [22]

$$
F_{2}(t)=\frac{3}{2 \pi^{2}}\left((1-t)_{+}\right)^{2} .
$$

For general $d \geq 3$, we will express $F_{d}(t)$ as an integral of a function given by the probability that a random lattice in $\mathbb{R}^{d-1}$ is disjoint from a cut paraboloid (cf. (3.75) below). This function has the following properties: The support of $F_{d}(t)$ is exactly the interval $0<t<\sqrt{\sigma_{d}(1,0)}$, where $\sigma_{d}(r, \alpha)$ is the same function as in (1.32). Furthermore $F_{d}(t)$ stays bounded from below 
as $t \rightarrow 0$; in fact the limit $F_{d}(0):=\lim _{t \rightarrow 0} F_{d}(t)>0$ exists; cf. Proposition 4.2 below. This number has a natural interpretation in relation with Theorem 1.11, The function $\Phi(\xi, w)$ may be extended to a continuous function on $\mathbb{R}_{>0} \times[0,1]$ (cf., e.g., [29, Lemma 2.3]). Since (1.40) holds uniformly with respect to $0 \leq w<1$ we conclude by letting $w \rightarrow 1$ :

$$
\Phi(\xi, 1)=\xi^{-2+\frac{2}{d}} F_{d}(0)+O(1)\left\{\begin{array}{ll}
\xi^{-2} & \text { if } d=2 \\
\xi^{-2} \log \xi & \text { if } d=3 \\
\xi^{-2} & \text { if } d \geq 4
\end{array}\right\}, \quad \text { as } \xi \rightarrow \infty .
$$

We prove Theorem 1.11 in Section 3, this proof also serves as a preparation for the proof of Theorem 1.7, which we give in Sections 4 5. On the other hand it is alternatively possible to obtain Theorem 1.11 (except we get a slightly worse error term when $d=3$ ) as a consequence of Theorem 1.7 and Theorem 1.9, via the formula (1.14). In fact this approach even gives an asymptotic formula for $\frac{\partial}{\partial \xi} \Phi(\xi, \boldsymbol{z})$ as $\xi \rightarrow \infty$; cf. Theorem 7.1 in Section 7 .

As a simple consequence of (1.14), Theorem 1.8 and Proposition 1.10, we obtain a precise understanding of the support of $\Phi(\xi, w)$ as $\xi \rightarrow \infty$. Recall that $F_{d}(t)>0$ if and only if $t<\sqrt{\sigma_{d}(1,0)}$.

Corollary 1.12. There is a continuous function $\xi_{0}:[0,1) \rightarrow \mathbb{R}_{>0}$ such that $\Phi(\xi, w)>0$ holds if and only if $\xi<\xi_{0}(w)$, and we have

$$
\xi_{0}(w)=\sigma_{d}(1,0)^{\frac{d}{4}}(1-w)^{-\frac{d}{2}}+O\left((1-w)^{1-\frac{d}{2}}\right)
$$

as $w \rightarrow 1^{-}$.

The above results directly yield asymptotics for the different limiting distributions of the free path length, (1.20), (1.21), (1.22). The first statement concerns the distribution function for the free path length of a trajectory with generic initial condition.

\section{Theorem 1.13.}

$$
\Phi(\xi)=\frac{\pi^{\frac{d-1}{2}}}{2^{d} d \Gamma\left(\frac{d+3}{2}\right) \zeta(d)} \xi^{-2}+O\left(\xi^{-2-\frac{2}{d}}\right) \quad \text { as } \xi \rightarrow \infty .
$$

This result sharpens the upper bound given by Bourgain, Golse and Wennberg [6] and the lower bound of Golse and Wennberg [16].

As to the distribution of the free path length between consecutive collisions, we have:

\section{Theorem 1.14.}

$$
\bar{\Phi}_{\mathbf{0}}(\xi)=\frac{2^{2-d}}{d(d+1) \zeta(d)} \xi^{-3}+O\left(\xi^{-3-\frac{2}{d}}\right)\left\{\begin{array}{ll}
1 & \text { if } d=2 \\
\log \xi & \text { if } d=3 \\
1 & \text { if } d \geq 4
\end{array}\right\} \quad \text { as } \xi \rightarrow \infty
$$

Finally, regarding the distribution of the free path length starting at a lattice point (with the scatterer removed), let us define $\delta_{d}^{*}(\mathfrak{Z})$ to be the maximal lattice packing density of any cylinder of the form $\mathfrak{Z}=(0, a) \times \mathcal{B}_{r}^{d-1}$ (cf. [23] or [17]); of course this number is independent of $a$ and $r$.

Proposition 1.15. Let $d \geq 2$ be given. Then $\Phi_{\mathbf{0}}(\xi)$ has compact support; $\Phi_{\mathbf{0}}(\xi)>0$ holds if and only if $0<\xi<\xi_{0}(0)=2^{d-1} v_{d-1}^{-1} \delta_{d}^{*}(\mathfrak{Z})$, where $\xi_{0}(w)$ is the function from Corollary 1.12.

Cf. [30] for a brief listing of what is known about $\delta_{d}^{*}(\mathfrak{Z})$ for general $d$, in particular note that $\delta_{d-1}^{*}(\mathcal{B}) \leq \delta_{d}^{*}(\mathfrak{Z}) \leq \delta_{d-1}(\mathcal{B})$, where $\delta_{d-1}(\mathcal{B})$ is the maximal packing density of balls in dimension $d-1$, and $\delta_{d-1}^{*}(\mathcal{B})$ is the corresponding lattice packing density. For dimension $d \leq 4$ we have explicitly:

- For $d=2$, we have $\delta_{2}^{*}(\mathfrak{Z})=1$, which implies $\xi_{0}(0)=1$; cf. [22, (30)].

- For $d=3$, we have $\delta_{3}^{*}(\mathfrak{Z})=\frac{\pi}{\sqrt{12}}$, which implies $\xi_{0}(0)=\frac{2}{\sqrt{3}}$.

- For $d=4$, we have $\delta_{4}^{*}(\mathfrak{Z})=\frac{\pi}{\sqrt{18}}\left(\right.$ cf. [31]), which implies $\xi_{0}(0)=\sqrt{2}$. 


\section{Asymptotics of $\Phi_{\mathbf{0}}(\xi, \boldsymbol{w}, \boldsymbol{z})$ FOR $\xi$ SMALL}

2.1. Recollection of definitions. We begin by recalling the formulas of the probability densities $\Phi(\xi, \boldsymbol{w})$ and $\Phi_{\mathbf{0}}(\xi, \boldsymbol{w}, \boldsymbol{z})$ derived in [20].

Throughout this paper we will write $G=\mathrm{SL}(d, \mathbb{R}), \Gamma=\mathrm{SL}(d, \mathbb{Z})$ and $X_{1}=\Gamma \backslash G$. We will view $X_{1}$ as the space of lattices in $\mathbb{R}^{d}$ of covolume one, by letting $M \in G$ correspond to the lattice $\mathbb{Z}^{d} M=\left\{\boldsymbol{v} M: \boldsymbol{v} \in \mathbb{Z}^{d}\right\}$. We define $\mu$ to be the measure on $X_{1}$ coming from Haar measure on $G$, normalized to be a probability measure. We will sometimes write $G^{(d)}, \Gamma^{(d)}$, $X_{1}^{(d)}$ and $\mu^{(d)}$ for $G, \Gamma, X_{1}$ and $\mu$, if we need to emphasize the dimension.

We denote by $\mathfrak{Z}\left(c_{1}, c_{2}, r\right)$ the cylinder

$$
\mathfrak{Z}\left(c_{1}, c_{2}, r\right)=\left(c_{1}, c_{2}\right) \times \mathcal{B}_{r}^{d-1}=\left\{\left(x_{1}, \ldots, x_{d}\right) \in \mathbb{R}^{d}: c_{1}<x_{1}<c_{2},\left\|\left(x_{2}, \ldots, x_{d}\right)\right\|<r\right\} .
$$

The function $\Phi: \mathbb{R}_{>0} \times \mathcal{B}_{1}^{d-1} \rightarrow[0,1]$ can be expressed as the probability that a random lattice in $X_{1}$ does not intersect the cylinder $\mathfrak{Z}(0, \xi, 1)+(0, \boldsymbol{w})$, i.e.,

$$
\Phi(\xi, \boldsymbol{w})=\mu\left(\left\{M \in X_{1}: \mathbb{Z}^{d} M \cap(\mathfrak{Z}(0, \xi, 1)+(0, \boldsymbol{w}))=\emptyset\right\}\right),
$$

cf. [20, (8.32)].

Next, for each $\boldsymbol{y} \in \mathbb{R}^{d} \backslash\{\mathbf{0}\}$ we set

$$
X_{1}(\boldsymbol{y})=\left\{M \in X_{1}: \boldsymbol{y} \in \mathbb{Z}^{d} M\right\} .
$$

This space carries a natural probability measure $\nu_{\boldsymbol{y}}$, the properties of which are discussed in [20, Sec. 7] and [29, Sec. 5]. The function $\Phi_{0}: \mathbb{R}_{>0} \times \mathcal{B}_{1}^{d-1} \times \mathcal{B}_{1}^{d-1} \rightarrow[0,1]$ is then given by

$$
\Phi_{\mathbf{0}}(\xi, \boldsymbol{w}, \boldsymbol{z})=\nu_{\boldsymbol{y}}\left(\left\{M \in X_{1}(\boldsymbol{y}): \mathbb{Z}^{d} M \cap(\mathfrak{Z}(0, \xi, 1)+(0, \boldsymbol{z}))=\emptyset\right\}\right),
$$

where $\boldsymbol{y}=(\xi, \boldsymbol{z}+\boldsymbol{w})$.

2.2. Proof of Theorem 1.1. We now give the proof of Theorem 1.1, viz. the fact that

$$
\zeta(d)^{-1}\left(1-2^{d-1} v_{d-1} \xi\right) \leq \Phi_{\mathbf{0}}(\xi, \boldsymbol{w}, \boldsymbol{z}) \leq \zeta(d)^{-1}
$$

for all $\xi>0, \boldsymbol{w}, \boldsymbol{z} \in \mathcal{B}_{1}^{d-1}$.

Using the $G$-invariance of $\nu_{\boldsymbol{y}}([20$, Lemma 7.2]) we may rewrite (2.4) as

$$
\Phi_{\mathbf{0}}(\xi, \boldsymbol{w}, \boldsymbol{z})=\nu_{\boldsymbol{y}}\left(\left\{M \in X_{1}(\boldsymbol{y}): \mathbb{Z}^{d} M \cap \mathfrak{Z}=\emptyset\right\}\right),
$$

with

$$
\mathfrak{Z}=\xi^{\frac{1}{d}}(\mathfrak{Z}(0,1,1)+(0, \boldsymbol{z})) ; \quad \boldsymbol{y}=\xi^{\frac{1}{d}}(1, \boldsymbol{z}+\boldsymbol{w}) .
$$

We will keep these choices of $\mathfrak{Z}$ and $\boldsymbol{y}$ from now on. Recall the splitting

$$
X_{1}(\boldsymbol{y})=\sqcup_{\boldsymbol{k} \in S} X_{1}(\boldsymbol{k}, \boldsymbol{y})
$$

where

$$
X_{1}(\boldsymbol{k}, \boldsymbol{y})=\left\{\Gamma M \in X_{1}: M \in G, \boldsymbol{k} M=\boldsymbol{y}\right\},
$$

and where we may take $S=\left\{k \boldsymbol{e}_{1}: k \in \mathbb{Z}_{>0}\right\}$, cf. [20, (7.11)-(7.12)]. Note that if $M \in \mathrm{SL}(d, \mathbb{R})$ is such that $k \boldsymbol{e}_{1} M=\boldsymbol{y}$ with $k \geq 2$ then $\boldsymbol{e}_{1} M=k^{-1} \boldsymbol{y} \in \mathfrak{Z}$; hence (2.5) may be rewritten as

$$
\Phi_{\mathbf{0}}(\xi, \boldsymbol{w}, \boldsymbol{z})=\nu_{\boldsymbol{y}}\left(\left\{M \in X_{1}\left(\boldsymbol{e}_{1}, \boldsymbol{y}\right): \mathbb{Z}^{d} M \cap \mathfrak{Z}=\emptyset\right\}\right) .
$$

Writing $\boldsymbol{y}=\left(y_{1}, \ldots, y_{d}\right)$ (thus $\left.y_{1}=\xi^{\frac{1}{d}}\right)$ we now set

$$
M^{\prime}=\left(\begin{array}{cccc}
y_{1} & y_{2} & \cdots & y_{d} \\
& y_{1}^{-\frac{1}{d-1}} & & \\
& & \ddots & \\
& & & y_{1}^{-\frac{1}{d-1}}
\end{array}\right) \in G
$$


so that $\boldsymbol{e}_{1} M^{\prime}=\boldsymbol{y}$. Then by [20, (7.15)] we have

$$
X_{1}\left(\boldsymbol{e}_{1}, \boldsymbol{y}\right)=((\Gamma(1) \cap H) \backslash H) M^{\prime}
$$

where

$$
H=\left\{g \in G: \boldsymbol{e}_{1} g=\boldsymbol{e}_{1}\right\}=\left\{\left(\begin{array}{cc}
1 & \mathbf{0} \\
{ }^{t} \boldsymbol{v} & A
\end{array}\right): \boldsymbol{v} \in \mathbb{R}^{d-1}, A \in G^{(d-1)}\right\},
$$

and the restriction of the measure $\nu_{\boldsymbol{y}}$ to $X_{1}\left(\boldsymbol{e}_{1}, \boldsymbol{y}\right)$ corresponds to the measure $\zeta(d)^{-1} d \boldsymbol{v} d \mu^{(d-1)}(A)$ on $(\Gamma(1) \cap H) \backslash H$ under (2.8). We also know that a fundamental domain for $(\Gamma(1) \cap H) \backslash H$ is given by $\left\{\left(\begin{array}{cc}1 & 0 \\ t_{\boldsymbol{v}} & A\end{array}\right): \boldsymbol{v} \in[0,1)^{d-1}, A \in \mathcal{F}_{d-1}\right\}$, where $\mathcal{F}_{d-1}$ is any fundamental domain for $\Gamma^{(d-1)} \backslash G^{(d-1)}$. Hence

$$
\Phi_{\mathbf{0}}(\xi, \boldsymbol{w}, \boldsymbol{z})=\zeta(d)^{-1} \int_{\mathcal{F}_{d-1}} \int_{[0,1)^{d-1}} I\left(\mathbb{Z}^{d}\left(\begin{array}{cc}
1 & \mathbf{0} \\
t_{\boldsymbol{v}} & A
\end{array}\right) M^{\prime} \cap \mathfrak{Z}=\emptyset\right) d \boldsymbol{v} d \mu^{(d-1)}(A),
$$

where $I(\cdot)$ is the indicator function. This relation immediately implies

$$
\Phi_{\mathbf{0}}(\xi, \boldsymbol{w}, \boldsymbol{z}) \leq \zeta(d)^{-1},
$$

thus proving the upper bound in (1.18). On the other hand, we claim that if $A$ is any matrix in $G^{(d-1)}$ with the property that $\|\boldsymbol{m} A\|>2 \xi^{\frac{1}{d-1}}$ for all $\boldsymbol{m} \in \mathbb{Z}^{d-1} \backslash\{\mathbf{0}\}$, then $\mathbb{Z}^{d}\left(\begin{array}{cc}1 & \mathbf{0} \\ \boldsymbol{t}_{\boldsymbol{v}} & A\end{array}\right) M^{\prime} \cap \mathfrak{Z}=\emptyset$ holds for all $\boldsymbol{v} \in \mathbb{R}^{d-1}$. Indeed, note that for any $\boldsymbol{m} \in \mathbb{Z}^{d-1}, j \in \mathbb{Z}$ we have

$$
(j, \boldsymbol{m})\left(\begin{array}{cc}
1 & \mathbf{0} \\
{ }^{\mathrm{t}} \boldsymbol{v} & A
\end{array}\right) M^{\prime}=\left(j+\boldsymbol{m}^{\mathrm{t}} \boldsymbol{v}, \boldsymbol{m} A\right) M^{\prime}=\left(j+\boldsymbol{m}^{\mathrm{t}} \boldsymbol{v}\right) \boldsymbol{y}+y_{1}^{-\frac{1}{d-1}}(0, \boldsymbol{m} A),
$$

and if this vector lies in $\mathfrak{Z}$ then its $\boldsymbol{e}_{1}$-component must lie in $\left(0, \xi^{\frac{1}{d}}\right)$, viz. $0<j+\boldsymbol{m}^{\mathrm{t}} \boldsymbol{v}<1$. Hence if we write $\alpha=j+\boldsymbol{m}^{\mathrm{t}} \boldsymbol{v}$ and let $p: \mathbb{R}^{d} \rightarrow \mathbb{R}^{d-1}$ be the projection $\left(x_{1}, \ldots, x_{d}\right) \mapsto\left(x_{2}, \ldots, x_{d}\right)$, it follows that

$$
\begin{aligned}
& \left\|p\left((j, \boldsymbol{m})\left(\begin{array}{rr}
1 & \mathbf{0} \\
\mathrm{t}_{\boldsymbol{v}} & A
\end{array}\right) M^{\prime}-\xi^{\frac{1}{d}}(0, \boldsymbol{z})\right)\right\|=\xi^{\frac{1}{d}}\left\|\alpha(\boldsymbol{z}+\boldsymbol{w})-\boldsymbol{z}+\xi^{-\frac{1}{d-1}} \boldsymbol{m} A\right\| \\
& \geq \xi^{\frac{1}{d}}\left(\xi^{-\frac{1}{d-1}}\|\boldsymbol{m} A\|-\alpha\|\boldsymbol{w}\|-(1-\alpha)\|\boldsymbol{z}\|\right)>\xi^{\frac{1}{d}}\left(\xi^{-\frac{1}{d-1}}\|\boldsymbol{m} A\|-1\right) .
\end{aligned}
$$

Now if $\boldsymbol{m} \in \mathbb{Z}^{d-1} \backslash\{\mathbf{0}\}$ and if $A$ has the stated property then the above distance is $>\xi^{\frac{1}{d}}$ and hence $(j, \boldsymbol{m})\left(\begin{array}{rr}1 & \mathbf{0} \\ \boldsymbol{t}_{\boldsymbol{v}} & A\end{array}\right) M^{\prime} \notin \mathfrak{Z}$. Furthermore if $\boldsymbol{m}=\mathbf{0}$ then $0<j+\boldsymbol{m}^{\mathrm{t}} \boldsymbol{v}<1$ is impossible and we again conclude $(j, \boldsymbol{m})\left(\begin{array}{cc}1 & 0 \\ \boldsymbol{t}_{\boldsymbol{v}} & A\end{array}\right) M^{\prime} \notin \mathfrak{Z}$. This proves the claim.

Using the claim just proved together with (2.10) we obtain

$$
\zeta(d)^{-1}-\Phi_{\mathbf{0}}(\xi, \boldsymbol{w}, \boldsymbol{z}) \leq \zeta(d)^{-1} \mu^{(d-1)}\left(\left\{A \in X_{1}^{(d-1)}: \mathbb{Z}^{d-1} A \cap \mathcal{B}_{2 \xi^{1 /(d-1)}}^{d-1} \neq\{\mathbf{0}\}\right\}\right),
$$

and by a well-known bound (cf. e.g. [29, Lemma 2.2] or [25, p. 167]) the right hand side is

$$
\leq \zeta(d)^{-1} \operatorname{vol}\left(\mathcal{B}_{2 \xi^{1 /(d-1)}}^{d-1}\right)=\zeta(d)^{-1} 2^{d-1} v_{d-1} \xi .
$$

This bound together with (2.11) complete the proof of Theorem 1.1.

2.3. A parametrization of $X_{1}\left(\boldsymbol{e}_{1}, \boldsymbol{y}\right)$ for $d=3$. We now turn to the case $d=3$ where we will prove the explicit formula for $\Phi_{\mathbf{0}}(\xi, \boldsymbol{w}, \boldsymbol{z})$ for $\xi$ small stated in Theorem 1.4. As a preparation we first give a parametrization of $X_{1}\left(\boldsymbol{e}_{1}, \boldsymbol{y}\right)$. Let $\boldsymbol{y}=\left(y_{1}, y_{2}, y_{3}\right) \in \mathbb{R}^{3} \backslash\{\mathbf{0}\}$ be given, and fix a matrix $M_{\boldsymbol{y}} \in G=G^{(3)}$ with $\boldsymbol{e}_{1} M_{\boldsymbol{y}}=\boldsymbol{y}$. Recall that

$$
X_{1}\left(\boldsymbol{e}_{1}, \boldsymbol{y}\right)=(\Gamma(1) \cap H) \backslash H M_{\boldsymbol{y}}
$$

(cf. [20, (7.15)]). Now an arbitrary matrix $M$ in $H M_{\boldsymbol{y}}$ has the form

$$
M=\left(\begin{array}{c}
\boldsymbol{y} \\
\boldsymbol{q} \\
\boldsymbol{p}
\end{array}\right)=\left(\begin{array}{lll}
y_{1} & y_{2} & y_{3} \\
q_{1} & q_{2} & q_{3} \\
p_{1} & p_{2} & p_{3}
\end{array}\right) \in H M_{\boldsymbol{y}}
$$


where $\boldsymbol{q}=\left(q_{1}, q_{2}, q_{3}\right)$ and $\boldsymbol{p}=\left(p_{1}, p_{2}, p_{3}\right)$ are two real vectors satisfying $\boldsymbol{q} \cdot(\boldsymbol{p} \times \boldsymbol{y})=1$, with " $\times$ " denoting vector product. Such a pair of vectors may be parametrized by $\langle\boldsymbol{p}, \boldsymbol{x}\rangle \in\left(\mathbb{R}^{3} \backslash \mathbb{R} \boldsymbol{y}\right) \times \mathbb{R}^{2}$, via the map

$$
\boldsymbol{q}=\boldsymbol{q}_{\boldsymbol{y}, \boldsymbol{p}}(\boldsymbol{x}):=x_{1} \boldsymbol{y}+x_{2} \boldsymbol{p}+\|\boldsymbol{p} \times \boldsymbol{y}\|^{-2} \boldsymbol{p} \times \boldsymbol{y} \quad\left(\text { where } \boldsymbol{x}=\left(x_{1}, x_{2}\right)\right) .
$$

Let us write $[\boldsymbol{p}, \boldsymbol{x}]_{\boldsymbol{y}}$ for the matrix $M$ obtained in this way. We have thus exhibited a (surjective) diffeomorphism

$$
\left(\mathbb{R}^{3} \backslash \mathbb{R} \boldsymbol{y}\right) \times \mathbb{R}^{2} \ni\langle\boldsymbol{p}, \boldsymbol{x}\rangle \mapsto[\boldsymbol{p}, \boldsymbol{x}]_{\boldsymbol{y}} \in H M_{\boldsymbol{y}}
$$

Note that the lattice corresponding to $[\boldsymbol{p}, \boldsymbol{x}]_{\boldsymbol{y}}$ is

$$
\mathbb{Z}^{3}[\boldsymbol{p}, \boldsymbol{x}]_{\boldsymbol{y}}=\mathbb{Z} \boldsymbol{y}+\mathbb{Z} \boldsymbol{p}+\mathbb{Z} \boldsymbol{q}_{\boldsymbol{y}, \boldsymbol{p}}(\boldsymbol{x}) .
$$

Recall that $\nu_{\boldsymbol{y}}$ is a probability measure on $X_{1}(\boldsymbol{y})$; by restriction this gives a measure on $X_{1}\left(\boldsymbol{e}_{1}, \boldsymbol{y}\right)$ (with $\left.\nu_{\boldsymbol{y}}\left(X_{1}\left(\boldsymbol{e}_{1}, \boldsymbol{y}\right)\right)=\zeta(d)^{-1}\right)$; we will write $\nu_{\boldsymbol{y}}$ also for the lift of this measure to $H M_{y}$.

Lemma 2.1. Given $\boldsymbol{y} \in \mathbb{R}^{3} \backslash\{\mathbf{0}\}$, the measure $\nu_{\boldsymbol{y}}$ on $H M_{\boldsymbol{y}}$ takes the following form in the $[\boldsymbol{p}, \boldsymbol{x}]_{\boldsymbol{y}}$-parametrization:

$$
d \nu_{\boldsymbol{y}}=\frac{6}{\pi^{2} \zeta(3)} d \boldsymbol{p} d \boldsymbol{x}
$$

Proof. Let $\mu_{H}$ be the Haar measure on $H$ normalized so that $\mu_{H}((\Gamma(1) \cap H) \backslash H)=1$; thus $d \mu_{H}=d \boldsymbol{v} d \mu^{(2)}(A)$ in the coordinates $\left(\begin{array}{cc}1 & \mathbf{0} \\ \boldsymbol{t}_{\boldsymbol{v}} & A\end{array}\right) \in H\left(\boldsymbol{v} \in \mathbb{R}^{2}, A \in G^{(2)}\right)$. By definition $\nu_{\boldsymbol{y}}$ on $H M_{\boldsymbol{y}}$ is the measure which corresponds to $\zeta(3)^{-1} \mu_{H}$ on $H$ under $h \mapsto h M_{\boldsymbol{y}}$ (cf. [20, Sec. 7.1]). Recall that both the set $H M_{\boldsymbol{y}}$ and the measure $\nu_{\boldsymbol{y}}$ on this set are independent of the choice of $M_{\boldsymbol{y}} \in G$ (subject to $\boldsymbol{e}_{1} M_{\boldsymbol{y}}=\boldsymbol{y}$ ). In particular if $\boldsymbol{y}$ is fixed and $R \in \mathrm{SO}(3)$ is a fixed rotation then we may choose $M_{\boldsymbol{y} R}=M_{\boldsymbol{y}} R$; using this together with $\boldsymbol{q}_{\boldsymbol{y} R, \boldsymbol{p} R}(\boldsymbol{x})=\boldsymbol{q}_{\boldsymbol{y}, \boldsymbol{p}}(\boldsymbol{x}) R$ and the fact that $\boldsymbol{p} \mapsto \boldsymbol{p} R$ preserves the Lebesgue measure $d \boldsymbol{p}$, one checks that if (2.17) holds for $\boldsymbol{y}$ then it also holds with $\boldsymbol{y}$ replaced by $\boldsymbol{y} R$.

Hence it suffices to prove (2.17) in the case $\boldsymbol{y}=y \boldsymbol{e}_{1}, y>0$, and we may then assume $M_{\boldsymbol{y}}$ to be the diagonal matrix $M_{\boldsymbol{y}}=\operatorname{diag}\left[y, y^{-\frac{1}{2}}, y^{-\frac{1}{2}}\right]$. In this case we compute that $h=$ $[\boldsymbol{p}, \boldsymbol{x}]_{\boldsymbol{y}} M_{\boldsymbol{y}}^{-1}=\left(\begin{array}{cc}1 & \mathbf{0} \\ { }^{\mathrm{t}} \boldsymbol{v} & A\end{array}\right) \in H$, with

$$
\boldsymbol{v}=\left(v_{1}, v_{2}\right)=y^{-1}\left(y x_{1}+x_{2} p_{1}, p_{1}\right)
$$

and, introducing variables $\nu>0$ and $\vartheta \in \mathbb{R} / 2 \pi \mathbb{Z}$ through $\left(p_{2}, p_{3}\right)=\nu^{-\frac{1}{2}}(\sin \vartheta, \cos \vartheta)$,

$$
A=y^{\frac{1}{2}}\left(\begin{array}{cc}
x_{2} p_{2}+\frac{p_{3}}{y\left(p_{2}^{2}+p_{3}^{2}\right)} & x_{2} p_{3}-\frac{p_{2}}{y\left(p_{2}^{2}+p_{3}^{2}\right)} \\
p_{2} & p_{3}
\end{array}\right)=\left(\begin{array}{cc}
1 & x_{2} \\
0 & 1
\end{array}\right)\left(\begin{array}{cc}
(\nu / y)^{\frac{1}{2}} & 0 \\
0 & (\nu / y)^{-\frac{1}{2}}
\end{array}\right)\left(\begin{array}{cc}
\cos \vartheta & -\sin \vartheta \\
\sin \vartheta & \cos \vartheta
\end{array}\right) .
$$

Note that the last matrix product is the Iwasawa decomposition of $G^{(2)}=\mathrm{SL}(2, \mathbb{R})$, in terms of which the (normalized) Haar measure takes the form

$$
d \mu^{(2)}(A)=\frac{3 y}{\pi^{2}} \frac{d x_{2} d \nu d \vartheta}{\nu^{2}}
$$

(cf. (3.4) below for the case of general $d$ ). Hence

$$
d \mu_{H}(h)=d \boldsymbol{v} d \mu^{(2)}(A)=\frac{3}{\pi^{2}} \frac{d p_{1} d x_{1} d x_{2} d \nu d \vartheta}{\nu^{2}}=\frac{6}{\pi^{2}} d p_{1} d p_{2} d p_{3} d x_{1} d x_{2}=\frac{6}{\pi^{2}} d \boldsymbol{p} d \boldsymbol{x} .
$$


2.4. Proof of Theorem 1.4. We keep $d=3$. Let $\xi>0$ and $\boldsymbol{w}, \boldsymbol{z} \in \mathcal{B}_{1}^{2}$ be given, and assume $\xi<\xi_{1}(\boldsymbol{w}, \boldsymbol{z})$. (The case $\xi=\xi_{1}(\boldsymbol{w}, \boldsymbol{z})$ then follows by continuity.) Recall that

$$
\Phi_{\mathbf{0}}(\xi, \boldsymbol{w}, \boldsymbol{z})=\nu_{\boldsymbol{y}}\left(\left\{M \in X_{1}\left(\boldsymbol{e}_{1}, \boldsymbol{y}\right): \mathbb{Z}^{3} M \cap \mathfrak{Z}=\emptyset\right\}\right)
$$

where $\mathfrak{Z}=\mathfrak{Z}(0, \xi, 1)+(0, \boldsymbol{z})$ and $\boldsymbol{y}=(\xi, \boldsymbol{z}+\boldsymbol{w})$. If $\boldsymbol{w} \neq \boldsymbol{z}$ then we let $\ell \subset \mathbb{R}^{2}$ be the line along the (unique) chord in $\mathcal{B}_{1}^{2}$ with has midpoint $\frac{1}{2}(\boldsymbol{w}-\boldsymbol{z})$, and let $V \subset \mathbb{R}^{3}$ be the affine plane

$$
V=\left\{\left(x_{1}, x_{2}, x_{3}\right): x_{1} \in \mathbb{R},\left(x_{2}, x_{3}\right) \in \boldsymbol{z}+\ell\right\} .
$$

Finally let $V^{+} \subset \mathbb{R}^{3}$ be that open halfspace which has boundary $V$ and which contains the axis of $\mathfrak{Z}$, viz. $\mathbb{R} \times\{\boldsymbol{z}\} \subset V^{+}$. If $\boldsymbol{w}=\boldsymbol{z}$ then we modify this definition by letting $\ell$ be an arbitrary diameter of $\mathcal{B}_{1}^{2}$ and $V^{+}$be any of the two open halfspaces determined by $V$.

Now consider the map

$$
J:\left(V^{+} \cap \mathfrak{Z} \backslash \mathbb{R} \boldsymbol{y}\right) \times[0,1)^{2} \ni\langle\boldsymbol{p}, \boldsymbol{x}\rangle \mapsto(\Gamma(1) \cap H)[\boldsymbol{p}, \boldsymbol{x}]_{\boldsymbol{y}} \in X_{1}\left(\boldsymbol{e}_{1}, \boldsymbol{y}\right) .
$$

Let us first prove that the image of $J$ equals, up to a set of $\nu_{\boldsymbol{y}}$-measure zero,

$$
\left\{M \in X_{1}\left(\boldsymbol{e}_{1}, \boldsymbol{y}\right): \mathbb{Z}^{3} M \cap \mathfrak{Z} \neq \emptyset\right\} .
$$

Indeed, every $M \in X_{1}\left(\boldsymbol{e}_{1}, \boldsymbol{y}\right)$ in the image of $J$ clearly satisfies $\mathbb{Z}^{3} M \cap \mathfrak{Z} \neq \emptyset$, since $\boldsymbol{p} \in$ $\mathbb{Z}^{3}[\boldsymbol{p}, \boldsymbol{x}]_{\boldsymbol{y}}$. On the other hand, if $M$ is any given element in $X_{1}\left(\boldsymbol{e}_{1}, \boldsymbol{y}\right)$ which satisfies $\mathbb{Z}^{3} M \cap \mathfrak{Z} \neq$ $\emptyset$ and also $\mathbb{Z}^{3} M \cap \mathfrak{Z} \cap V=\emptyset$ (this latter condition holds for $\nu_{\boldsymbol{y}}$-almost all $M \in X_{1}\left(\boldsymbol{e}_{1}, \boldsymbol{y}\right)$ ), then we will prove that $M=J(\langle\boldsymbol{p}, \boldsymbol{x}\rangle)$ for some $\langle\boldsymbol{p}, \boldsymbol{x}\rangle \in\left(V^{+} \cap \mathfrak{Z} \backslash \mathbb{R} \boldsymbol{y}\right) \times[0,1)^{2}$. To this end, among the finitely many points in $\mathbb{Z}^{3} M \cap \mathfrak{Z}$ we pick one which has minimal distance to the line $\mathbb{R} \boldsymbol{y}$, and call it $\boldsymbol{p}^{\prime}$. Note that $\boldsymbol{p}^{\prime} \notin V$, due to our assumption $\mathbb{Z}^{3} M \cap \mathfrak{Z} \cap V=\emptyset$. If $\boldsymbol{p}^{\prime} \in V^{+}$then set $\boldsymbol{p}:=\boldsymbol{p}^{\prime}$; otherwise set $\boldsymbol{p}:=\boldsymbol{y}-\boldsymbol{p}^{\prime}$. In both cases $\boldsymbol{p} \in V^{+} \cap \mathfrak{Z}$ must hold (this follows from the fact that among the two regions into which the line $\ell$ splits the unit disc $\mathcal{B}_{1}^{2}$, the smaller one is mapped into the larger one by reflection in the point $\frac{1}{2}(\boldsymbol{w}-\boldsymbol{z})$ ); also $\boldsymbol{p} \in \mathbb{Z}^{3} M$ and $\boldsymbol{p}$ has the same distance as $\boldsymbol{p}^{\prime}$ to the line $\mathbb{R} \boldsymbol{y}$. Note also that $\boldsymbol{p} \notin \mathbb{R} \boldsymbol{y}$, since $\boldsymbol{p} \in \mathfrak{Z}$ forces $0<\boldsymbol{p} \cdot \boldsymbol{e}_{1}<\xi$ while all points in $\mathbb{R} \boldsymbol{y} \cap \mathbb{Z}^{3} M=\mathbb{Z} \boldsymbol{y}$ have $\boldsymbol{e}_{1}$-coordinates in $\mathbb{Z} \xi$. Now $\mathbb{Z}^{3} M \cap(\mathbb{R} \boldsymbol{y}+\mathbb{R} \boldsymbol{p})=\mathbb{Z} \boldsymbol{y}+\mathbb{Z} \boldsymbol{p}$, for otherwise there would exist a point $\boldsymbol{r} \in \mathbb{Z}^{3} M \backslash\{\boldsymbol{0}, \boldsymbol{y}, \boldsymbol{p}\}$ lying in the triangle $\triangle \mathbf{0 y p}$ (the convex hull of $\mathbf{0}, \boldsymbol{y}, \boldsymbol{p})$; this point $\boldsymbol{r}$ would belong to $\mathfrak{Z}$ since $\mathfrak{Z}$ is convex, and $\boldsymbol{r}$ would also lie closer to $\mathbb{R} \boldsymbol{y}$ than $\boldsymbol{p}$ does, thus causing a contradiction. It follows from $\mathbb{Z}^{3} M \cap(\mathbb{R} \boldsymbol{y}+\mathbb{R} \boldsymbol{p})=\mathbb{Z} \boldsymbol{y}+\mathbb{Z} \boldsymbol{p}$ that there exists a point $\boldsymbol{q} \in \mathbb{Z}^{3} M$ satisfying $\mathbb{Z}^{3} M=\mathbb{Z} \boldsymbol{y}+\mathbb{Z} \boldsymbol{p}+\mathbb{Z} \boldsymbol{q}$ and $\boldsymbol{q} \cdot(\boldsymbol{p} \times \boldsymbol{y})=1$. Then $\boldsymbol{q}=\boldsymbol{q}_{\boldsymbol{y}, \boldsymbol{p}}(\boldsymbol{x})$ for some $\boldsymbol{x} \in \mathbb{R}^{2}$ (cf. (2.15)), and replacing $\boldsymbol{q}$ by $\boldsymbol{q}+n_{1} \boldsymbol{y}+n_{2} \boldsymbol{p}$ with appropriate $n_{1}, n_{2} \in \mathbb{Z}$ we may assume $\boldsymbol{x} \in[0,1)^{2}$. Now $\mathbb{Z}^{3} M=\mathbb{Z} \boldsymbol{y}+\mathbb{Z} \boldsymbol{p}+\mathbb{Z} \boldsymbol{q}_{\boldsymbol{y}, \boldsymbol{p}}(\boldsymbol{x})=\mathbb{Z}[\boldsymbol{p}, \boldsymbol{x}]_{\boldsymbol{y}}$ (cf. (2.16) $)$, and this implies $M=(\Gamma(1) \cap H)[\boldsymbol{p}, \boldsymbol{x}]_{\boldsymbol{y}}=J(\langle\boldsymbol{p}, \boldsymbol{x}\rangle)$, thus completing the proof of our claim.

Next we prove that $J$ is injective. Thus assume $J(\langle\boldsymbol{p}, \boldsymbol{x}\rangle)=J\left(\left\langle\boldsymbol{p}^{\prime}, \boldsymbol{x}^{\prime}\right\rangle\right)$ for some $\boldsymbol{p}, \boldsymbol{p}^{\prime} \in$ $V^{+} \cap \mathfrak{Z} \backslash \mathbb{R} \boldsymbol{y}, \boldsymbol{x}, \boldsymbol{x}^{\prime} \in[0,1)^{2}$. Then

$$
\mathbb{Z} \boldsymbol{y}+\mathbb{Z} \boldsymbol{p}+\mathbb{Z} \boldsymbol{q}_{\boldsymbol{y}, \boldsymbol{p}}(\boldsymbol{x})=\mathbb{Z} \boldsymbol{y}+\mathbb{Z} \boldsymbol{p}^{\prime}+\mathbb{Z} \boldsymbol{q}_{\boldsymbol{y}, \boldsymbol{p}^{\prime}}\left(\boldsymbol{x}^{\prime}\right)
$$

(cf. (2.16) ). We now claim that

$$
\mathfrak{Z} \cap\left(\mathbb{R} \boldsymbol{y}+\mathbb{R} \boldsymbol{p}+n \boldsymbol{q}_{\boldsymbol{y}, \boldsymbol{p}}(\boldsymbol{x})\right)=\emptyset \quad \text { for all } n \in \mathbb{Z} \backslash\{0\} .
$$

Indeed, assume that there exists a point $\boldsymbol{r} \in \mathfrak{Z} \cap\left(\mathbb{R} \boldsymbol{y}+\mathbb{R} \boldsymbol{p}+n \boldsymbol{q}_{\boldsymbol{y}, \boldsymbol{p}}(\boldsymbol{x})\right)$, for some non-zero integer $n$. Then the tetrahedron with vertices $\mathbf{0}, \boldsymbol{y}, \boldsymbol{p}, \boldsymbol{r}$ has volume $\frac{1}{6}|n| \geq \frac{1}{6}$ (cf. (2.15)); hence after a scaling and a translation we obtain a tetrahedron which is contained in the closed cylinder $\overline{\mathfrak{Z}(0,1,1)}=[0,1] \times \overline{\mathcal{B}_{1}^{2}}$, which has one vertex at $(0,-\boldsymbol{z})$ and another at $(1, \boldsymbol{w})$, and which has volume $\geq(6 \xi)^{-1}$. This is impossible, since $\xi<\xi_{1}(\boldsymbol{w}, \boldsymbol{z})$ (recall the definition of $\xi_{1}(\boldsymbol{w}, \boldsymbol{z})$ given just before the statement of Theorem 1.4); hence (2.22) is proved.

Now $\boldsymbol{p}^{\prime} \in \mathbb{Z} \boldsymbol{y}+\mathbb{Z} \boldsymbol{p}+\mathbb{Z} \boldsymbol{q}_{\boldsymbol{y}, \boldsymbol{p}}(\boldsymbol{x}), \boldsymbol{p}^{\prime} \in \mathfrak{Z}$ and (2.22) imply $\boldsymbol{p}^{\prime} \in \mathbb{Z} \boldsymbol{y}+\mathbb{Z} \boldsymbol{p}$. Similarly $\boldsymbol{p} \in \mathbb{Z} \boldsymbol{y}+\mathbb{Z} \boldsymbol{p}^{\prime}$. It follows that $\boldsymbol{p}^{\prime}=\varepsilon \boldsymbol{p}+m \boldsymbol{y}$ for some $\varepsilon= \pm 1, m \in \mathbb{Z}$. If $\varepsilon=-1$ then since both $\boldsymbol{e}_{1} \cdot \boldsymbol{p}$ and $\boldsymbol{e}_{1} \cdot \boldsymbol{p}^{\prime}$ lie in the interval $(0, \xi)$ we must have $m=1$, viz. $\boldsymbol{p}^{\prime}=\boldsymbol{y}-\boldsymbol{p}$. It follows that the midpoint of the line segment between $\boldsymbol{p}$ and $\boldsymbol{p}^{\prime}$ is $\frac{1}{2} \boldsymbol{y} \in V$, and this contradicts the assumption that 
both $\boldsymbol{p}$ and $\boldsymbol{p}^{\prime}$ lie in $V^{+}$. Hence we must have $\varepsilon=1$; and by again using $\boldsymbol{e}_{1} \cdot \boldsymbol{p}, \boldsymbol{e}_{1} \cdot \boldsymbol{p}^{\prime} \in(0, \xi)$ we get $m=0$, viz. $\boldsymbol{p}^{\prime}=\boldsymbol{p}$. Finally using (2.15), (2.21) and $\boldsymbol{x}, \boldsymbol{x}^{\prime} \in[0,1)^{2}$ we see that also $\boldsymbol{x}^{\prime}=\boldsymbol{x}$ must hold. This completes the proof that $J$ is injective.

It follows that $J$ is a diffeomorphism of $\left(V^{+} \cap \mathfrak{Z} \backslash \mathbb{R} \boldsymbol{y}\right) \times(0,1)^{2}$ onto an open subset of full $\left(\nu_{\boldsymbol{y}^{-}}\right)$measure in $\left\{M \in X_{1}\left(\boldsymbol{e}_{1}, \boldsymbol{y}\right): \mathbb{Z}^{3} M \cap \mathfrak{Z} \neq \emptyset\right\}$. Hence by (2.18) and Lemma 2.1,

$$
\Phi_{\mathbf{0}}(\xi, \boldsymbol{w}, \boldsymbol{z})=\nu_{\boldsymbol{y}}\left(X_{1}\left(\boldsymbol{e}_{1}, \boldsymbol{y}\right)\right)-\frac{6}{\pi^{2} \zeta(3)} \int_{\mathfrak{Z} \cap V^{+}} \int_{(0,1)^{2}} d \boldsymbol{p} d \boldsymbol{x}=\frac{1}{\zeta(3)}-\frac{6}{\pi^{2} \zeta(3)} F\left(\frac{1}{2}\|\boldsymbol{w}-\boldsymbol{z}\|\right) \xi .
$$

This completes the proof of Theorem 1.4.

Using methods that are beyond the scope of the present paper we are able to prove that the function $\xi_{1}(\boldsymbol{w}, \boldsymbol{z})$ gives the true range of validity of the formula (1.26), i.e. for any $\boldsymbol{w}, \boldsymbol{z} \in \mathcal{B}_{1}^{2}$, there exist triples $\left\langle\xi, \boldsymbol{w}^{\prime}, \boldsymbol{z}^{\prime}\right\rangle$ arbitrarily near $\left\langle\xi_{1}(\boldsymbol{w}, \boldsymbol{z}), \boldsymbol{w}, \boldsymbol{z}\right\rangle$ at which (1.26) fails.

We next give sharp lower and upper bounds on $\xi_{1}(\boldsymbol{w}, \boldsymbol{z})$. Recall that we defined (on p. 7) $\xi_{1}(\boldsymbol{w}, \boldsymbol{z})$ for $\boldsymbol{w}, \boldsymbol{z} \in \mathcal{B}_{1}^{2}$ as $\xi_{1}(\boldsymbol{w}, \boldsymbol{z})=(6 V)^{-1}$, where $V$ is the largest possible volume of a tetrahedron which is contained in the closed cylinder $\overline{\mathfrak{Z}(0,1,1)}=[0,1] \times \overline{\mathcal{B}_{1}^{2}}$ and which has one vertex at $(0,-\boldsymbol{z})$ and another at $(1, \boldsymbol{w})$. By applying the same formula to arbitrary $\boldsymbol{w}, \boldsymbol{z} \in \overline{\mathcal{B}_{1}^{2}}$ we obtain an extension of $\xi_{1}$ to a continuous function $\overline{\mathcal{B}_{1}^{2}} \times \overline{\mathcal{B}_{1}^{2}} \rightarrow \mathbb{R}$; we will write $\xi_{1}$ also for this extension.

Lemma 2.2. We have

$$
\frac{1}{4} \leq \xi_{1}(\boldsymbol{w}, \boldsymbol{z}) \leq 1 \quad \text { for all } \boldsymbol{w}, \boldsymbol{z} \in \overline{\mathcal{B}_{1}^{2}}
$$

Here $\xi_{1}(\boldsymbol{w}, \boldsymbol{z})=\frac{1}{4}$ holds if and only if $\|\boldsymbol{w}\|=\|\boldsymbol{z}\|=1$ and $\boldsymbol{w} \cdot \boldsymbol{z}=0$; and $\xi_{1}(\boldsymbol{w}, \boldsymbol{z})=1$ holds if and only if $\boldsymbol{w}=\boldsymbol{z}=\mathbf{0}$.

Note that this implies that $\frac{1}{4}<\xi_{1}(\boldsymbol{w}, \boldsymbol{z}) \leq 1$ for all $\boldsymbol{w}, \boldsymbol{z} \in \mathcal{B}_{1}^{2}$, both bounds being sharp.

Proof. Let $V_{0}$ be the maximal volume of a tetrahedron contained in $\overline{\mathfrak{Z}(0,1,1)}$. This volume is clearly attained; let us fix $T \subset \overline{\mathfrak{Z}(0,1,1)}$ to be a tetrahedron of volume $V_{0}$. By a simple variational argument, varying the vertices of $T$ one at a time, we see that $T$ may be continuously deformed, keeping its volume $V_{0}$ fixed, into a tetrahedron $T^{\prime}$ which has all its vertices lying on the two circles $\{0\} \times S_{1}^{1}$ and $\{1\} \times S_{1}^{1}$. Clearly each of these circles must contain at least one vertex. If each circle contains two vertices, say $\boldsymbol{a}, \boldsymbol{b} \in\{0\} \times \mathrm{S}_{1}^{1}$ and $\boldsymbol{c}, \boldsymbol{d} \in\{1\} \times \mathrm{S}_{1}^{1}$, then the same type of variational argument also shows that both vectors $\boldsymbol{c}-\boldsymbol{e}_{1}$ and $\boldsymbol{d}-\boldsymbol{e}_{1}$ must be orthogonal to $\boldsymbol{b}-\boldsymbol{a}$, and both $\boldsymbol{a}, \boldsymbol{b}$ must be orthogonal to $\boldsymbol{d}-\boldsymbol{c}$. In other words, the line segment $\boldsymbol{a} \boldsymbol{b}$ must be a diameter of $\{0\} \times \mathrm{S}_{1}^{1}$, and the line segment $\boldsymbol{c} \boldsymbol{d}$ must be that diameter of $\{1\} \times \mathrm{S}_{1}^{1}$ whose direction is orthogonal to $\boldsymbol{a} \boldsymbol{b}$. We compute that any such tetrahedron $T^{\prime}$ has volume $\frac{2}{3}$. On the other hand if one circle contains three of the vertices, say $\boldsymbol{a}, \boldsymbol{b}, \boldsymbol{c}$, then $\triangle \boldsymbol{a b c}$ must be an equilateral triangle, and $\operatorname{vol}\left(T^{\prime}\right)=\frac{\sqrt{3}}{4}<\frac{2}{3}$, a contradiction. It follows that $V_{0}=\frac{2}{3}$. Note also that for a tetrahedron $T^{\prime}$ with $\boldsymbol{a}, \boldsymbol{b} \in\{0\} \times \mathrm{S}_{1}^{1}$ and $\boldsymbol{c}, \boldsymbol{d} \in\{1\} \times \mathrm{S}_{1}^{1}$ and volume $V_{0}=\frac{2}{3}$ (i.e. with $\boldsymbol{a} \boldsymbol{b}$ and $\boldsymbol{c} \boldsymbol{d}$ being diameters whose directions are orthogonal), any perturbation of $\boldsymbol{a}$ inside $\overline{\mathfrak{Z}(0,1,1)}$ with $\boldsymbol{b}, \boldsymbol{c}, \boldsymbol{d}$ fixed makes vol $\left(T^{\prime}\right)$ strictly smaller (since the plane through $\boldsymbol{a}$ orthogonal to $(\boldsymbol{b}-\boldsymbol{d}) \times(\boldsymbol{c}-\boldsymbol{d})$ contains only $\boldsymbol{a}$ in its intersection with $\overline{\mathfrak{Z}(0,1,1)})$. This implies that also the original tetrahedron $T$ necessarily had two vertices on $\{0\} \times \mathrm{S}_{1}^{1}$ and two vertices on $\{1\} \times \mathrm{S}_{1}^{1}$, i.e. we have proved that a tetrahedron with vertices $\boldsymbol{a}, \boldsymbol{b}, \boldsymbol{c}, \boldsymbol{d} \in \overline{\mathfrak{Z}(0,1,1)}$ attains the maximal volume $V_{0}=\frac{2}{3}$ if and only if, up to a renaming of the vertices, $\boldsymbol{a} \boldsymbol{b}$ is a diameter of $\{0\} \times \mathrm{S}_{1}^{1}$ and $\boldsymbol{c} \boldsymbol{d}$ is a diameter of $\{0\} \times \mathrm{S}_{1}^{1}$ whose direction is orthogonal to $\boldsymbol{a} \boldsymbol{b}$.

This result immediately implies that $\xi_{1}(\boldsymbol{w}, \boldsymbol{z}) \geq \frac{1}{4}$ for all $\boldsymbol{w}, \boldsymbol{z} \in \overline{\mathcal{B}_{1}^{2}}$, with equality if and only if $\|\boldsymbol{w}\|=\|\boldsymbol{z}\|=1$ and $\boldsymbol{w} \cdot \boldsymbol{z}=0$.

Next, given any $\boldsymbol{w}, \boldsymbol{z} \in \overline{\mathcal{B}_{1}^{2}}$, let us consider the tetrahedron $T$ which has vertices $(0,-\boldsymbol{z})$, $(1, \boldsymbol{w}),(1, \boldsymbol{a}),(1, \boldsymbol{b})$, with $\boldsymbol{a}, \boldsymbol{b} \in \overline{\mathcal{B}_{1}^{1}}$ chosen so as to maximize the area of the triangle $\triangle \boldsymbol{a b} \boldsymbol{w}$. 
A simple variational argument, varying $\boldsymbol{a}$ and $\boldsymbol{b}$ one at a time, shows that these $\boldsymbol{a}, \boldsymbol{b}$ must satisfy $\boldsymbol{a}, \boldsymbol{b} \in \mathrm{S}_{1}^{1}$ and $\boldsymbol{a} \cdot(\boldsymbol{b}-\boldsymbol{w})=\boldsymbol{b} \cdot(\boldsymbol{a}-\boldsymbol{w})=0$, and $\boldsymbol{a}$ and $\mathbf{0}$ must lie on the same side of the line $\boldsymbol{b} \boldsymbol{w}$, and $\boldsymbol{b}$ and $\mathbf{0}$ must lie on the same side of the line $\boldsymbol{a} \boldsymbol{w}$. If $\boldsymbol{w} \neq \mathbf{0}$ then this determines $\boldsymbol{a} \boldsymbol{b}$ to be the unique chord of $\mathrm{S}_{1}^{1}$ with midpoint $-\frac{f(\|\boldsymbol{w}\|)}{\|\boldsymbol{w}\|} \boldsymbol{w}$ where $f(w)=\frac{2}{\sqrt{w^{2}+8}+w}$, and we get

$$
\operatorname{vol}(T)=\sup _{\alpha \in[0,1]} \frac{1}{3}(\alpha+\|\boldsymbol{w}\|) \sqrt{1-\alpha^{2}}=\frac{1}{3}(f(\|\boldsymbol{w}\|)+\|\boldsymbol{w}\|) \sqrt{1-f(\|\boldsymbol{w}\|)^{2}} .
$$

On the other hand if $\boldsymbol{w}=\mathbf{0}$ then the maximal area of $\triangle \boldsymbol{a b} \boldsymbol{w}$ is attained if and only if $\boldsymbol{a}, \boldsymbol{b} \in \mathrm{S}_{1}^{1}$ are orthogonal, and then $\operatorname{vol}(T)=\frac{1}{6}$, i.e. (2.23) still holds. It is clear from the first expression in (2.23) that $\operatorname{vol}(T)$ is a strictly increasing function of $\|\boldsymbol{w}\| \in[0,1]$; in particular we have $\operatorname{vol}(T) \geq \frac{1}{6}$, with equality if and only if $\boldsymbol{w}=\mathbf{0}$. This implies that $\xi_{1}(\boldsymbol{w}, \boldsymbol{z}) \leq 1$, where equality is possible only if $\boldsymbol{w}=\mathbf{0}$. Similarly, by instead taking $T$ to have vertices $(0,-\boldsymbol{z}),(1, \boldsymbol{w}),(0, \boldsymbol{a})$, $(0, \boldsymbol{b})$, we see that $\xi_{1}(\boldsymbol{w}, \boldsymbol{z})=1$ can only hold if $\boldsymbol{z}=\mathbf{0}$.

To complete the proof of the lemma it now only remains to prove that $\xi_{1}(\mathbf{0}, \mathbf{0})=1$. Thus let $T$ be a tetrahedron with vertices $\boldsymbol{a}, \boldsymbol{b}, \boldsymbol{c}, \boldsymbol{d}$ and of maximal volume subject to $\boldsymbol{c}=(0, \mathbf{0})=\mathbf{0}$, $\boldsymbol{d}=(1, \mathbf{0})=\boldsymbol{e}_{1}$ and $\boldsymbol{a}, \boldsymbol{b} \in \overline{\mathfrak{Z}(0,1,1)}$. By the same type of variational argument as in the first half of this proof we may assume $\boldsymbol{a}, \boldsymbol{b} \in\left(\{0\} \times \mathrm{S}_{1}^{1}\right) \cup\left(\{1\} \times \mathrm{S}_{1}^{1}\right)$. If $\boldsymbol{a}, \boldsymbol{b}$ lie on the same circle then as in the discussion leading to (2.23) we get $\operatorname{vol}(T)=\frac{1}{6}$. On the other hand if $\boldsymbol{a}, \boldsymbol{b}$ lie on distinct circles, say $\boldsymbol{a} \in\{0\} \times \mathrm{S}_{1}^{1}$ and $\boldsymbol{b} \in\{1\} \times \mathrm{S}_{1}^{1}$, then similarly as in the first half of this proof we must have $\left(\boldsymbol{b}-\boldsymbol{e}_{1}\right) \cdot \boldsymbol{a}=0$, and this implies $\operatorname{vol}(T)=\frac{1}{6}$, again. Hence $\xi_{1}(\mathbf{0}, \mathbf{0})=1$.

2.5. Explicit formulas for $\Phi(\xi, \boldsymbol{w}), \bar{\Phi}_{\mathbf{0}}(\xi), \Phi_{\mathbf{0}}(\xi)$ and $\Phi(\xi)$ for $d=3, \xi$ small.

Proof of Corollary 1.5. Using (1.14) and Theorem 1.4 we have, whenever $0<\xi \leq \inf _{\boldsymbol{z} \in \mathcal{B}_{1}^{2}} \xi_{1}(\boldsymbol{w}, \boldsymbol{z})$,

$$
\Phi(\xi, \boldsymbol{w})=1-\int_{0}^{\xi} \int_{\mathcal{B}_{1}^{2}} \Phi_{\mathbf{0}}(\eta, \boldsymbol{w}, \boldsymbol{z}) d \boldsymbol{z} d \eta=1-\frac{\pi}{\zeta(3)} \xi+\frac{3}{\pi^{2} \zeta(3)}\left(\int_{\mathcal{B}_{1}^{2}} F\left(\frac{1}{2}\|\boldsymbol{w}-\boldsymbol{z}\|\right) d \boldsymbol{z}\right) \xi^{2} .
$$

Substituting $\boldsymbol{z}=\boldsymbol{w}+r(\cos \omega, \sin \omega)$ we get, writing $w=\|\boldsymbol{w}\| \in[0,1)$ :

$\int_{\mathcal{B}_{1}^{2}} F\left(\frac{1}{2}\|\boldsymbol{w}-\boldsymbol{z}\|\right) d \boldsymbol{z}=2 \pi \int_{0}^{1-w} F\left(\frac{1}{2} r\right) r d r+2 \int_{1-w}^{1+w} F\left(\frac{1}{2} r\right) \arccos \left(\frac{w^{2}+r^{2}-1}{2 w r}\right) r d r=2 G(w)$

(cf. (1.28) ). Hence (1.27) holds.

To see that $G(w)$ is a strictly increasing function of $w$ we may e.g. note that, for $0<w<1$,

$$
G^{\prime}(w)=\int_{1-w}^{1+w} \frac{\left(r^{2}-1-w^{2}\right) r}{w \sqrt{(1+w)^{2}-r^{2}} \sqrt{r^{2}-(1-w)^{2}}} F\left(\frac{1}{2} r\right) d r
$$

and here

$\int_{1-w}^{1+w} \frac{\left(r^{2}-1-w^{2}\right) r}{w \sqrt{(1+w)^{2}-r^{2}} \sqrt{r^{2}-(1-w)^{2}}} d r=\left[-\frac{1}{2 w} \sqrt{(1+w)^{2}-r^{2}} \sqrt{r^{2}-(1-w)^{2}}\right]_{r=1-w}^{r=1+w}=0$, with $\frac{\left(r^{2}-1-w^{2}\right) r}{w \sqrt{(1+w)^{2}-r^{2}} \sqrt{r^{2}-(1-w)^{2}}}$ being negative for $r \in\left(1-w, \sqrt{1-w^{2}}\right)$ and positive for $r \in$ $\left(\sqrt{1-w^{2}}, 1+w\right)$. Furthermore $F\left(\frac{1}{2} r\right)$ is strictly increasing for $0 \leq r \leq 2$. Hence it follows from (2.24) that $G^{\prime}(w)>0$ for all $0<w<1$, as desired.

The formulas $G(0)=\frac{\pi(4 \pi+3 \sqrt{3})}{16}$ and $G(1)=\frac{5}{16} \pi^{2}+1$ follow by straighforward computations directly from the definitions; for example for the computation of $G(1)$ one uses the fact that the following is a primitive function of $F\left(\frac{1}{2} r\right) r \arccos \left(\frac{r}{2}\right)$ :

$$
\begin{aligned}
\frac{1}{4}\left(3-2 r^{2}\right) \arccos \left(\frac{1}{2} r\right)^{2}+\left(\frac{1}{16} r\left(r^{2}+6\right) \sqrt{4-r^{2}}\right. & \left.+\frac{1}{2} \pi\left(r^{2}-2\right)\right) \arccos \left(\frac{1}{2} r\right) \\
& +\frac{1}{64}\left(16+12 r^{2}+r^{4}\right)-\frac{1}{4} \pi r \sqrt{4-r^{2}} .
\end{aligned}
$$


To complete the proof of Corollary 1.5 it now only remains to prove that

$$
\inf _{\boldsymbol{z} \in \mathcal{B}_{1}^{2}} \xi_{1}(\boldsymbol{w}, \boldsymbol{z})=\min \left(\frac{1}{2(1+\|\boldsymbol{w}\|)}, \frac{2}{3 \sqrt{3}}\right) .
$$

It is clear from the definition of $\xi_{1}(\boldsymbol{w}, \boldsymbol{z})$ that $\inf _{\boldsymbol{z} \in \mathcal{B}_{1}^{2}} \xi_{1}(\boldsymbol{w}, \boldsymbol{z})=(6 V)^{-1}$, where $V$ is the largest possible volume of a tetrahedron which is contained in the closed cylinder $[0,1] \times \overline{\mathcal{B}_{1}^{2}}$ and which has one vertex at $(1, \boldsymbol{w})$. By simple variational arguments of the same type as in the proof of Lemma 2.2 we see that this volume $V$ is attained either for a tetrahedron whose three other vertices lie on $\{0\} \times \mathrm{S}_{1}^{1}$ and form an equilateral triangle, or else for a tetrahedron with vertices $(1, \boldsymbol{w}), \boldsymbol{a}, \boldsymbol{b}, \boldsymbol{c}$ where $\boldsymbol{a} \in\{1\} \times \mathrm{S}_{1}^{1}$, the line segment between $(1, \boldsymbol{w})$ and $\boldsymbol{a}$ contains the point $(1, \mathbf{0})$, and $\boldsymbol{b} \boldsymbol{c}$ is that diameter of $\{0\} \times \mathrm{S}_{1}^{1}$ whose direction is orthogonal to $\boldsymbol{a}-(1, \boldsymbol{w})$. A tetrahedron of the first type has volume $\frac{\sqrt{3}}{4}$ and a tetrahedron of the second type has volume $\frac{1}{3}(1+\|\boldsymbol{w}\|)$; hence (2.25) follows.

Proof of Corollary 1.6. By (1.20), Theorem 1.4 and Lemma 2.2 we have, whenever $0<\xi \leq \frac{1}{4}$,

$$
\bar{\Phi}_{\mathbf{0}}(\xi)=\frac{1}{\pi} \int_{\mathcal{B}_{1}^{2}} \int_{\mathcal{B}_{1}^{2}} \Phi_{\mathbf{0}}(\xi, \boldsymbol{w}, \boldsymbol{z}) d \boldsymbol{w} d \boldsymbol{z}=\frac{\pi}{\zeta(3)}-\frac{6}{\pi^{3} \zeta(3)}\left(\int_{\mathcal{B}_{1}^{2}} \int_{\mathcal{B}_{1}^{2}} F\left(\frac{1}{2}\|\boldsymbol{w}-\boldsymbol{z}\|\right) d \boldsymbol{z} d \boldsymbol{w}\right) \xi
$$

Writing $\boldsymbol{z}=\boldsymbol{w}+r(\cos \omega, \sin \omega)$ and $w=\|\boldsymbol{w}\|$ as in the proof of Corollary 1.5 we have

$$
\begin{array}{r}
\int_{\mathcal{B}_{1}^{2}} \int_{\mathcal{B}_{1}^{2}} F\left(\frac{1}{2}\|\boldsymbol{w}-\boldsymbol{z}\|\right) d \boldsymbol{z} d \boldsymbol{w}=2 \pi \int_{0}^{1} \int_{0}^{1+w} F\left(\frac{1}{2} r\right)\left\{\begin{array}{ll}
2 \pi & \text { if } r<1-w \\
2 \arccos \left(\frac{w^{2}+r^{2}-1}{2 w r}\right) & \text { if } r \geq 1-w
\end{array}\right\} r d r w d w \\
=2 \pi \int_{0}^{2} F\left(\frac{1}{2} r\right) \int_{\max (0, r-1)}^{1}\left\{\begin{array}{ll}
2 \pi & \text { if } w<1-r \\
2 \arccos \left(\frac{w^{2}+r^{2}-1}{2 w r}\right) & \text { if } w \geq 1-r
\end{array}\right\} w d w r d r .
\end{array}
$$

Here the inner integral equals $2\left(\pi-F\left(\frac{1}{2} r\right)\right)$, and thus the above expression evaluates to

$$
4 \pi \int_{0}^{2} F\left(\frac{1}{2} r\right)\left(\pi-F\left(\frac{1}{2} r\right)\right) r d r=4 \pi\left(\frac{1}{8} \pi^{2}+\frac{2}{3}\right) .
$$

(The last step is by a straightforward computation, which is much simplified by re-using facts from the computation of $G(0)$ and $G(1)$ in the proof of Corollary 1.5.) Hence we obtain the formula for $\bar{\Phi}_{\mathbf{0}}(\xi)$ stated in Corollary [1.6. Furthermore, using $\Phi(\xi)=\pi\left(1-\int_{0}^{\xi} \bar{\Phi}_{\mathbf{0}}(\eta) d \eta\right.$ ) (cf. (1.21) and (1.14)), we also obtain the stated formula for $\Phi(\xi)$, for all $0<\xi \leq \frac{1}{4}$.

Finally, by (1.22) and Theorem 1.4 we have, whenever $0<\xi \leq \inf _{\boldsymbol{w} \in \mathcal{B}_{1}^{2}} \xi_{1}(\boldsymbol{w}, \mathbf{0})$,

$$
\Phi_{\mathbf{0}}(\xi)=\frac{\pi}{\zeta(3)}-\frac{12}{\pi \zeta(3)}\left(\int_{0}^{1} F\left(\frac{1}{2} r\right) r d r\right) \xi=\frac{\pi}{\zeta(3)}-\frac{3(4 \pi+3 \sqrt{3})}{4 \pi \zeta(3)} \xi .
$$

This gives the stated formula for $\Phi_{\mathbf{0}}(\xi)$, since $\inf _{\boldsymbol{w} \in \mathcal{B}_{1}^{2}} \xi_{1}(\boldsymbol{w}, \mathbf{0})=\inf _{\boldsymbol{z} \in \mathcal{B}_{1}^{2}} \xi_{1}(\mathbf{0}, \boldsymbol{z})=\frac{2}{3 \sqrt{3}}$ by (2.25).

2.6. Numerical computations for $d=3$. We now describe how the graphs of $\Phi(\xi)$ and $\Phi_{0}(\xi)$ in Figures 3 and 4 were obtained. For $d=3$ and any $0 \leq \alpha<\beta$ we have, by [20, (4.3) and (3.8)],

$\int_{\alpha}^{\beta} \Phi(\xi) d \xi=\int_{X_{1}^{(3)}} \int_{\mathbb{R}^{3} / \mathbb{Z}^{3}} I\left(\left\{\left(\mathbb{Z}^{3}+\boldsymbol{x}\right) M \cap \mathfrak{Z}(0, \alpha, 1)=\emptyset,\left(\mathbb{Z}^{3}+\boldsymbol{x}\right) M \cap \mathfrak{Z}(0, \beta, 1) \neq \emptyset\right\}\right) d \boldsymbol{x} d \mu(M)$.

The first curve in Figure 3 was obtained by using this formula to evaluate $\delta^{-1} \int_{n \delta}^{(n+1) \delta} \Phi(\xi) d \xi$ for $\delta=0.02, n=0,1, \ldots, 99$; taking this to be an approximation of $\Phi\left(\left(n+\frac{1}{2}\right) \delta\right)$, and drawing the piecewise linear curve connecting these points. We stress that $\Phi(\xi)$ as well as $\Phi_{\mathbf{0}}(\xi)$ are known to be continuous and decreasing functions, cf. (1.21), (1.22) and [29, Lemma 7.11]. The second curve in Figure 3 was obtained similarly, using $\delta=0.05, n=20, \ldots, 99$. 
In order to evaluate (2.26) numerically, the integral over $X_{1}$ was replaced by an average over the Hecke points corresponding to a large prime $p$, shifted by a fixed rotation; viz. $M=p^{-\frac{1}{3}} T k$ with fixed $k \in \mathrm{SO}(3)$ and $T$ running through the set

$$
S(p)=\left\{\left(\begin{array}{lll}
p & & \\
& 1 & \\
& & 1
\end{array}\right),\left(\begin{array}{lll}
1 & a & \\
& p & \\
& & 1
\end{array}\right),\left(\begin{array}{lll}
1 & & a \\
& 1 & b \\
& & p
\end{array}\right): a, b \in\{0,1,2, \ldots, p-1\}\right\} .
$$

Also the integral over $\mathbb{R}^{3} / \mathbb{Z}^{3}$ was replaced by an average over the $m^{3}$ points in $\boldsymbol{x}_{0}+m^{-1} \mathbb{Z}^{3} / \mathbb{Z}^{3}$, for some fixed $\boldsymbol{x}_{0} \in \mathbb{R}^{3} / \mathbb{Z}^{3}$ and $m \in \mathbb{Z}_{>0}$. For any fixed $\boldsymbol{x}_{0}, k$, this approximation is known to approach the correct value as $p, m \rightarrow \infty$; cf., e.g., [24], 9], [10. In our numerical experiments we noted that the rate of convergence seems to be improved by taking $\boldsymbol{x}_{0}$ irrational and also taking $k$ to be "sufficiently generic". For the curves in Figure 3 we used $p=1511, m=20$,

$$
k=\left(\begin{array}{ccc}
\cos (1 / 2) & \sin (1 / 2) & 0 \\
-\sin (1 / 2) & \cos (1 / 2) & 0 \\
0 & 0 & 1
\end{array}\right)\left(\begin{array}{ccc}
1 & 0 & 0 \\
0 & \cos 1 & \sin 1 \\
0 & -\sin 1 & \cos 1
\end{array}\right)\left(\begin{array}{ccc}
\cos (3 / 2) & \sin (3 / 2) & 0 \\
-\sin (3 / 2) & \cos (3 / 2) & 0 \\
0 & 0 & 1
\end{array}\right)
$$

and $\boldsymbol{x}_{0}=(\sqrt{2}, \sqrt{3}, \sqrt{5})$. We did not prove any error bounds for our approximation; however as an indication of the error we mention that for $n$ with $(n+1) \delta \leq \frac{1}{4}$, the value which we obtained for $\delta^{-1} \int_{n \delta}^{(n+1) \delta} \Phi(\xi) d \xi$ always differed by less than 0.003 from the known exact values of both $\delta^{-1} \int_{n \delta}^{(n+1) \delta} \Phi(\xi) d \xi$ and $\Phi\left(\left(n+\frac{1}{2}\right) \delta\right)$ (cf. Corollary 1.6). Also repeated runs with other choices of $p, m, k, \boldsymbol{x}_{0}$ indicate that our values for $\delta^{-1} \int_{n \delta}^{(n+1) \delta} \Phi(\xi) d \xi$ are correct to within an absolute error $<0.003$, for all $n$.

Similarly, for Figure 4 we used the formula ([20, (4.3) and (3.8)])

$$
\int_{\alpha}^{\beta} \Phi_{\mathbf{0}}(\xi) d \xi=\int_{X_{1}^{(3)}} I\left(\left\{\mathbb{Z}^{3} M \cap \mathfrak{Z}(0, \alpha, 1)=\emptyset, \mathbb{Z}^{3} M \cap \mathfrak{Z}(0, \beta, 1) \neq \emptyset\right\}\right) d \mu(M)
$$

to evaluate $\int_{n \delta}^{(n+1) \delta} \Phi_{0}(\xi) d \xi$ for $\delta=0.02, n=0,1, \ldots, 59$. For this case our experiments suggest that, for a given number of sample points $M=p^{-\frac{1}{3}} T k$, we get a significantly better approximation of the $X_{1}$-integral by running $T$ through a random subset of $S(p)$ with $p$ quite large, than by running $T$ through all of $S(p)$ for a $p$ of more modest size. The curve in Figure 4 was obtained by using $p=10^{9}+7, k$ as above, and letting $T$ run through $5.4 \cdot 10^{8}$ randomly choosen points from $S(p)$. Comparison against the known $\Phi_{0^{-}}$-values for $\xi \leq 0.38$. (cf. Corollary 1.6), as well as comparisons versus the results of using other random seeds and/or other choices of $k$ and $p$, indicate that our values for $\delta^{-1} \int_{n \delta}^{(n+1) \delta} \Phi_{\mathbf{0}}(\xi) d \xi$ are correct to within an absolute error $<0.005$.

Regarding the support of $\Phi_{0}(\xi)$, recall from Proposition 1.15 and the ensuing comments that $\Phi_{\mathbf{0}}(\xi)>0$ holds if and only if $\xi<\xi_{0}(0)=2 / \sqrt{3}=1.15470 \ldots$. Our numerics show that the function $\Phi_{\mathbf{0}}(\xi)$ approaches zero quite quickly as $\xi$ approaches $2 / \sqrt{3}$, and the largest value of $\sup \left\{\alpha>0: \mathbb{Z}^{3} M \cap \mathfrak{Z}(0, \alpha, 1)=\emptyset\right\}$ which we saw among our sample points $M$ was $\alpha \approx 1.132$.

\section{Asymptotics FOR $\Phi(\xi, \boldsymbol{w})$ AS $\xi \rightarrow \infty$}

In this section we prove Theorem 1.11 on the asymptotic size of $\Phi(\xi, w)$ as $\xi \rightarrow \infty$. Along the way we prove several lemmas which will also be useful later in our proof of Theorem 1.7 in Sections 4 5. Note that a second proof of Theorem 1.11 will be given in Section 7, where we deduce Theorem 1.11 as a consequence of Theorem 1.7, using the integration formula (1.14). 
3.1. Preliminaries: Iwasawa decomposition and Siegel domains. Recall that we write $G=\mathrm{SL}(d, \mathbb{R})$. Let $A$ be the subgroup of diagonal matrices with positive entries

$$
\mathrm{a}(a)=\left(\begin{array}{ccc}
a_{1} & & \\
& \ddots & \\
& & a_{d}
\end{array}\right) \in G, \quad a_{j}>0,
$$

and let $N$ be the subgroup of upper triangular matrices

$$
\mathrm{n}(u)=\left(\begin{array}{cccc}
1 & u_{12} & \cdots & u_{1 d} \\
& \ddots & \ddots & \vdots \\
& & \ddots & u_{d-1, d} \\
& & & 1
\end{array}\right) \in G .
$$

Every element $M \in G$ has a unique Iwasawa decomposition

$$
M=\mathrm{n}(u) \mathrm{a}(a) \mathrm{k},
$$

with $\mathrm{k} \in \mathrm{SO}(d)$. In these coordinates the Haar measure takes the form ([13, p. 172])

$$
d \mu(M)=\frac{2^{d-1} \pi^{d(d+1) / 4}}{\prod_{j=1}^{d} \Gamma\left(\frac{j}{2}\right) \prod_{j=2}^{d} \zeta(j)} \rho(a) d \mathrm{n}(u) d \mathrm{a}(a) d \mathrm{k}
$$

where $d \mathrm{n}, d \mathrm{a}, d \mathrm{k}$, are (left and right) Haar measures of $N, A, \mathrm{SO}(d)$, normalized by $d \mathrm{n}(u)=$ $\prod_{1 \leq j<k \leq d} d u_{j k}, d \mathrm{a}(a)=\prod_{j=1}^{d-1}\left(a_{j}^{-1} d a_{j}\right)$ and $\int_{\mathrm{SO}(d)} d \mathrm{k}=1$. For $\rho(a)$ one has

$$
\rho(a)=\prod_{1 \leq i<j \leq d} \frac{a_{j}}{a_{i}}=\prod_{j=1}^{d} a_{j}^{2 j-d-1} .
$$

We set $\mathcal{F}_{N}=\left\{u: u_{j k} \in\left(-\frac{1}{2}, \frac{1}{2}\right], 1 \leq j<k \leq d\right\}$; then $\left\{\mathrm{n}(u): u \in \mathcal{F}_{N}\right\}$ is a fundamental region for $(\Gamma \cap N) \backslash N$. We define the following Siegel set:

$$
\mathcal{S}_{d}:=\left\{\mathrm{n}(u) \mathrm{a}(a) \mathrm{k} \in G: u \in \mathcal{F}_{N}, 0<a_{j+1} \leq \frac{2}{\sqrt{3}} a_{j}(j=1, \ldots, d-1), \mathrm{k} \in \operatorname{SO}(d)\right\} .
$$

It is known that $\mathcal{S}_{d}$ contains a fundamental region for $X_{1}=\Gamma \backslash G$, and on the other hand $\mathcal{S}_{d}$ is contained in a finite union of fundamental regions for $X_{1}([5])$.

Given $M=\mathrm{n}(u) \mathrm{a}(a) \mathrm{k} \in G$, its row vectors are

$$
\boldsymbol{b}_{k}=\left(0, \ldots, 0, a_{k}, a_{k+1} u_{k, k+1}, \ldots, a_{d} u_{k, d}\right) \mathrm{k}, \quad k=1, \ldots, d .
$$

Thus $\boldsymbol{b}_{1}, \ldots, \boldsymbol{b}_{d}$ is a basis of the lattice $\mathbb{Z}^{d} M$. If $M \in \mathcal{S}_{d}$ then we see that, for all $k$,

$$
\left\|\boldsymbol{b}_{k}\right\| \leq \sum_{j=1}^{d} a_{j} \leq c_{1} a_{1}, \quad \text { with } c_{1}=c_{1}^{(d)}:=\sum_{j=0}^{d-1}(2 / \sqrt{3})^{j} .
$$

Throughout the paper we will let $c_{1}, c_{2}, \ldots$ denote certain constants which we fix once and for all and which only depend on $d$ (or in some cases are absolute); the $d$-dependence will mostly be suppressed but if necessary it will be made explicit by writing " $c_{j}^{(d)}$ ".

The bound (3.8) implies that if $M \in \mathcal{S}_{d}$ and if the lattice $\mathbb{Z}^{d} M$ has empty intersection with a large ball, then $a_{1}$ must be large:

Lemma 3.1. For any $M=\mathrm{n}(u) \mathrm{a}(a) \mathrm{k} \in \mathcal{S}_{d}$ such that the lattice $\mathbb{Z}^{d} M$ is disjoint from some ball of radius $R$ in $\mathbb{R}^{d}$, we have $a_{1} \gg R$.

Proof. Choose $h_{1}, \ldots, h_{d} \in \mathbb{R}$ so that $\boldsymbol{p}=h_{1} \boldsymbol{b}_{1}+\ldots+h_{d} \boldsymbol{b}_{d}$ is the center of the given ball. Let $n_{j}$ be the integer nearest to $h_{j}$. Then $n_{1} \boldsymbol{b}_{1}+\ldots+n_{d} \boldsymbol{b}_{d}$ is a lattice point of $\mathbb{Z}^{d} M$, and has distance $\leq \frac{1}{2}\left(\left\|\boldsymbol{b}_{1}\right\|+\ldots+\left\|\boldsymbol{b}_{d}\right\|\right) \ll a_{1}$ to $\boldsymbol{p}$. This distance must be $>R$; hence $a_{1} \gg R$. 
3.2. A parametrization of lattices with $a_{1}$ large. The set of lattices in $X_{1}$ which have a representative $M \in \mathcal{S}_{d}$ with $a_{1}$ larger than some large fixed number $A$, may in an approximate sense be parametrized by the set $(A, \infty) \times\left(\mathrm{S}_{1}^{d-1} / \pm\right) \times\left(\mathbb{R}^{d-1} / \mathbb{Z}^{d-1}\right) \times X_{1}^{(d-1)}$. In this section we prove a version of this fact, Lemma 3.4 below, which we will make use of several times.

Let us fix a function $f$ (smooth except possibly at one point, say) $\mathrm{S}_{1}^{d-1} \rightarrow \mathrm{SO}(d)$ such that $\boldsymbol{e}_{1} f(\boldsymbol{v})=\boldsymbol{v}$ for all $\boldsymbol{v} \in S^{d-1}$. Given $M=\mathrm{n}(u) \mathrm{a}(a) \mathrm{k} \in G$, the matrices $\mathrm{n}(u), \mathrm{a}(a)$ and $\mathrm{k}$ can be split uniquely as

$$
\mathrm{n}(u)=\left(\begin{array}{cc}
1 & \boldsymbol{u} \\
\mathbf{t} & \mathrm{n}(\underset{\sim}{u})
\end{array}\right) ; \quad \mathrm{a}(a)=\left(\begin{array}{cc}
a_{1} & \mathbf{0} \\
\mathrm{t}_{\mathbf{0}} & a_{1}^{-\frac{1}{d-1}} \mathrm{a}(\underset{\sim}{a)})
\end{array}\right) ; \quad \mathrm{k}=\left(\begin{array}{cc}
1 & \mathbf{0} \\
\mathbf{0} & \underset{\sim}{\mathrm{k}}
\end{array}\right) f(\boldsymbol{v})
$$

where $\boldsymbol{u} \in \mathbb{R}^{d-1}, \mathrm{n}(\underset{\sim}{u}) \in N^{(d-1)}, a_{1}>0, \mathrm{a}(\underset{\sim}{a}) \in A^{(d-1)}$ and $\underset{\sim}{\mathrm{k}} \in \mathrm{SO}(d-1), \boldsymbol{v} \in \mathrm{S}_{1}^{d-1}$. We set

$$
\underset{\sim}{\sim}=\mathrm{n}(\underset{\sim}{u}) \mathrm{a}(\underset{\sim}{a}) \underset{\sim}{\mathrm{k}} \in G^{(d-1)} \quad\left(\text { recall } G^{(d-1)}=\mathrm{SL}(d-1, \mathbb{R})\right) .
$$

In this way we get a bijection between $G$ and $\mathbb{R}_{>0} \times \mathrm{S}_{1}^{d-1} \times \mathbb{R}^{d-1} \times G^{(d-1)}$; we write $M=$ $\left[a_{1}, \boldsymbol{v}, \boldsymbol{u}, \underset{\sim}{M}\right]$ for the element in $G$ corresponding to the 4-tuple $\left\langle a_{1}, \boldsymbol{v}, \boldsymbol{u}, M \underset{\sim}{\sim}\right\rangle \in \mathbb{R}_{>0} \times \mathrm{S}_{1}^{d-1} \times \mathbb{R}^{d-1} \times$ $G^{(d-1)}$. In particular note that

$$
\begin{aligned}
\mathcal{S}_{d}=\left\{\left[a_{1}, \boldsymbol{v}, \boldsymbol{u}, \underset{\sim}{M}\right]\right. & \left.\in G: \underset{\sim}{\sim} \in \mathcal{S}_{d-1}, a_{1} \leq \frac{2}{\sqrt{3}} a_{1}^{\frac{d}{d-1}}, \boldsymbol{u} \in\left(-\frac{1}{2}, \frac{1}{2}\right]^{d-1}\right\} \\
& \subset\left\{\left[a_{1}, \boldsymbol{v}, \boldsymbol{u}, \underset{\sim}{M}\right] \in G: \underset{\sim}{M} \in \mathcal{S}_{d-1}, \boldsymbol{u} \in\left(-\frac{1}{2}, \frac{1}{2}\right]^{d-1}\right\} .
\end{aligned}
$$

One checks by a straightforward computation using (3.4) that the Haar measure $\mu$ takes the following form in the parametrization $M=\left[a_{1}, \boldsymbol{v}, \boldsymbol{u}, \underset{\sim}{M}\right]$ :

$$
d \mu(M)=\zeta(d)^{-1} d \mu^{(d-1)}(\underset{\sim}{M}) d \boldsymbol{u} d \boldsymbol{v} \frac{d a_{1}}{a_{1}^{d+1}},
$$

where $d \boldsymbol{v}$ is the $(d-1)$-dimensional volume measure on $\mathrm{S}_{1}^{d-1}$. Note that all of the above claims are valid also for $d=2$, with the natural interpretation that $\mathcal{S}_{1}=\operatorname{SL}(1, \mathbb{R})=\{1\}$ with $\mu^{(1)}(\{1\})=1$. We will also need to know the explicit expression of the lattice $\mathbb{Z}^{d} M$ in terms of $a_{1}, \boldsymbol{v}, \boldsymbol{u}, \underset{\sim}{M}$ : One computes that, for any $\boldsymbol{m} \in \mathbb{Z}^{d-1}$ and $n \in \mathbb{Z}$,

$$
(n, \boldsymbol{m}) M=n a_{1} \boldsymbol{v}+a_{1}^{-\frac{1}{d-1}}(0, n \boldsymbol{u a}(\underset{\sim}{a}) \underset{\sim}{\mathrm{k}}+\underset{\boldsymbol{m}}{\underset{\sim}{M}}) f(\boldsymbol{v}) .
$$

In particular we always have

$$
\mathbb{Z}^{d} M \subset \bigsqcup_{n \in \mathbb{Z}}\left(n a_{1} \boldsymbol{v}+\boldsymbol{v}^{\perp}\right)
$$

Let us fix a subset $\mathrm{S}_{ \pm}^{d-1} \subset \mathrm{S}_{1}^{d-1} \cap\left\{x_{1} \geq 0\right\}$ which contains exactly one of the vectors $\boldsymbol{v}$ and $-\boldsymbol{v}$ for every $\boldsymbol{v} \in S_{1}^{d-1}$. Let us also fix a (set theoretical, measurable) fundamental region $\mathcal{F}_{d-1} \subset \mathcal{S}_{d-1}$ for $\Gamma^{(d-1)} \backslash G^{(d-1)}$. Now for $A>1$ we set

$$
\mathcal{G}_{A}:=\left\{\left[a_{1}, \boldsymbol{v}, \boldsymbol{u}, \underset{\sim}{\sim}\right] \in G: a_{1}>A, \boldsymbol{v} \in \mathrm{S}_{ \pm}^{d-1}, \boldsymbol{u} \in\left(-\frac{1}{2}, \frac{1}{2}\right]^{d-1}, \underset{\sim}{M} \in \mathcal{F}_{d-1}\right\} .
$$

Lemma 3.2. If $M, M^{\prime} \in \mathcal{G}_{A}$ satisfy $M^{\prime}=\gamma M$ for some $\gamma \in \operatorname{SL}(d, \mathbb{Z})$, and if $a_{2}, a_{2}^{\prime}<$ $\left(c_{1}^{(d-1)}\right)^{-1} A$ in the Iwasawa decompositions $M=\mathrm{n}(u) \mathrm{a}(a) \mathrm{k}, M^{\prime}=\mathrm{n}\left(u^{\prime}\right) \mathrm{a}\left(a^{\prime}\right) \mathrm{k}^{\prime}$, then $M=M^{\prime}$.

Proof. Assume that $M=\mathrm{n}(u) \mathrm{a}(a) \mathrm{k}=\left[a_{1}, \boldsymbol{v}, \boldsymbol{u}, \underset{\sim}{M}\right]$ and $M^{\prime}=\mathrm{n}\left(u^{\prime}\right) \mathrm{a}\left(a^{\prime}\right) \mathrm{k}^{\prime}=\left[a_{1}^{\prime}, \boldsymbol{v}^{\prime}, \boldsymbol{u}^{\prime}, M_{\sim}^{\prime}\right]$ satisfy the assumptions of the lemma. Then $\mathbb{Z}^{d} \widetilde{M}^{\prime}=\mathbb{Z}^{d} \gamma M=\mathbb{Z}^{d} M$, and this lattice has a basis $\boldsymbol{b}_{1}, \ldots, \boldsymbol{b}_{d}$ (cf. (3.7)), and also a basis $\boldsymbol{b}_{1}^{\prime}, \ldots, \boldsymbol{b}_{d}^{\prime}$, the row vectors of $M^{\prime}$. Now for each $\boldsymbol{m}=\left(m_{1}, \ldots, m_{d}\right) \in \mathbb{Z}^{d}$ with $m_{1} \neq 0$ the lattice vector $\boldsymbol{m} M$ has length $\geq a_{1}$, since $(\boldsymbol{m} M) \cdot\left(\boldsymbol{e}_{1} \mathrm{k}\right)=m_{1} a_{1}$. On the other hand, by a similar argument as in (3.8), using $\underset{\sim}{M^{\prime}} \in$ $\mathcal{F}_{d-1} \subset \mathcal{S}_{d-1}$, we have $\left\|\boldsymbol{b}_{j}^{\prime}\right\| \leq c_{1}^{(d-1)} a_{2}^{\prime}<A<a_{1}$ for each $j \geq 2$; thus $\boldsymbol{b}_{j}^{\prime} \in \mathbb{Z} \boldsymbol{b}_{2}+\cdots+\widetilde{\mathbb{Z}} \boldsymbol{b}_{d}$. Similarly $\boldsymbol{b}_{j} \in \mathbb{Z} \boldsymbol{b}_{2}^{\prime}+\cdots+\mathbb{Z} \boldsymbol{b}_{d}^{\prime}$ for each $j \geq 2$. Hence $\mathbb{Z} \boldsymbol{b}_{2}^{\prime}+\cdots+\mathbb{Z} \boldsymbol{b}_{d}^{\prime}=\mathbb{Z} \boldsymbol{b}_{2}+\cdots+\mathbb{Z} \boldsymbol{b}_{d}$. Let $\Pi \subset \mathbb{R}^{d}$ be the hyperplane spanned by this set of vectors. Now $a_{1}^{-1}=\operatorname{vol}\left(\Pi /\left(\mathbb{Z} \boldsymbol{b}_{2}+\cdots+\mathbb{Z} \boldsymbol{b}_{d}\right)\right)$ 
((d-1)-dimensional volume), and similarly for $a_{1}^{\prime}$; hence $a_{1}=a_{1}^{\prime}$. Also $\boldsymbol{v}=\boldsymbol{e}_{1} f(\boldsymbol{v})=\boldsymbol{e}_{1} \mathrm{k} \in \Pi^{\perp}$ and similarly $\boldsymbol{v}^{\prime} \in \Pi^{\perp}$; hence since $\boldsymbol{v}, \boldsymbol{v}^{\prime} \in S_{ \pm}^{d-1}$ we conclude $\boldsymbol{v}=\boldsymbol{v}^{\prime}$. Next by (3.13), if $\iota$ denotes the embedding $\iota: \mathbb{R}^{d-1} \ni\left(x_{1}, \ldots, x_{d-1}\right) \mapsto\left(0, x_{1}, \ldots, x_{d-1}\right) \in \mathbb{R}^{d}$, then

$$
\iota\left(\mathbb{Z}^{d-1} \stackrel{M}{\sim}\right)=a_{1}^{\frac{1}{d-1}} \iota\left(\mathbb{Z}^{d-1}\right) M f(\boldsymbol{v})^{-1}=a_{1}^{\frac{1}{d-1}}\left(\mathbb{Z} \boldsymbol{b}_{2}+\cdots+\mathbb{Z} \boldsymbol{b}_{d}\right) f(\boldsymbol{v})^{-1},
$$

and similarly for $\mathbb{Z}^{d-1} M^{\prime}$; hence $\mathbb{Z}^{d-1} \underset{\sim}{\sim}=\mathbb{Z}^{d-1} M^{\prime}$, and since $\underset{\sim}{\sim}, M^{\prime} \in \mathcal{F}_{d-1}$ we conclude $\underset{\sim}{M}=M_{\sim}^{\prime}$. Hence also $\underset{\sim}{\sim}=\underline{\sim}^{\prime}, \underset{\sim}{a}=\underset{\sim}{a^{\prime}}$ and $\underset{\sim}{\mathrm{k}}={\underset{\sim}{\mathbf{k}^{\prime}}}^{\sim}$ (in an obvious notation, cf. (3.10)), and we now also obtain $\mathrm{a}(a)=\mathrm{a}\left(a^{\prime}\right)$ and $\mathrm{k}=\mathrm{k}^{\prime}$, so that $M^{\prime}=\gamma M$ implies $\mathrm{n}\left(u^{\prime}\right)=\gamma \mathrm{n}(u)$. But $\mathrm{n}\left(u^{\prime}\right)=\gamma \mathrm{n}(u)$ together with ${\underset{\sim}{u}}^{\prime}=\underset{\sim}{u}$ and $\boldsymbol{u}, \boldsymbol{u}^{\prime} \in\left(-\frac{1}{2}, \frac{1}{2}\right]^{d-1}$ imply $\gamma=I$, thus $M^{\prime}=M$.

Lemma 3.3. If $M=\mathrm{n}(u) \mathrm{a}(a) \mathrm{k} \in \mathcal{S}_{d}$ has $a_{1}>A$ and $a_{2}<\left(c_{1}^{(d-1)}\right)^{-1} A$, then there is some $\gamma \in \Gamma$ such that $\gamma M \in \mathcal{G}_{A} \cap \mathcal{S}_{d}$.

Proof. Take any $M=\mathrm{n}(u) \mathrm{a}(a) \mathrm{k}=\left[a_{1}, \boldsymbol{v}, \boldsymbol{u}, \underset{\sim}{M}\right] \in \mathcal{S}_{d}$ with $a_{1}>A$ and $a_{2}<\left(c_{1}^{(d-1)}\right)^{-1} A$. We write $\underset{\sim}{M}=\mathrm{n}(\underset{\sim}{u}) \mathrm{a}(\underset{\sim}{a}) \mathbf{\sim}$ as usual. If $\boldsymbol{v} \notin \mathrm{S}_{ \pm}^{d-1}$ then we replace $M$ with $\gamma D M$, where $D=\operatorname{diag}[-1,1, \ldots, 1,-1] \in \Gamma$ and $\gamma \in \Gamma \cap N$ is chosen so that $\gamma D \operatorname{n}(u) D \in \mathcal{F}_{N}$; this new $M$ lies in $\mathcal{S}_{d}$ and has the same a $(a)$ component as before but $\boldsymbol{v}$ negated. Hence from now on we may assume $\boldsymbol{v} \in S_{ \pm}^{d-1}$.

Take $\gamma_{1} \in \Gamma^{(d-1)}$ so that $\gamma_{1} \underset{\sim}{\sim} \in \mathcal{F}_{d-1}$; let the Iwasawa decomposition of this matrix be $\gamma_{1} \underset{\sim}{M}=\mathrm{n}\left({\underset{\sim}{u}}^{\prime}\right) \mathrm{a}\left({\underset{\sim}{\prime}}^{\prime}\right) \mathfrak{\sim}^{\prime}$, and let $\boldsymbol{w}$ be the unique vector in $\mathbb{Z}^{d-1}$ with $\boldsymbol{w} \mathrm{n}\left({\underset{\sim}{u}}^{\prime}\right) \in-\boldsymbol{u a}(\underset{\sim}{a}) \underset{\sim}{\mathrm{kk}^{\prime}}{ }^{-1} \mathrm{a}\left({\underset{\sim}{a}}^{\prime}\right)^{-1}+$ $\left(-\frac{1}{2}, \frac{1}{2}\right]^{d-1}$. Set $\gamma=\left(\begin{array}{cc}1 & \boldsymbol{w} \gamma_{1} \\ t_{0} & \gamma_{1}\end{array}\right) \in \Gamma$.

We now claim $\gamma M \in \mathcal{G}_{A} \cap \mathcal{S}_{d}$. To prove this, first note that $\gamma M$ has Iwasawa decomposition

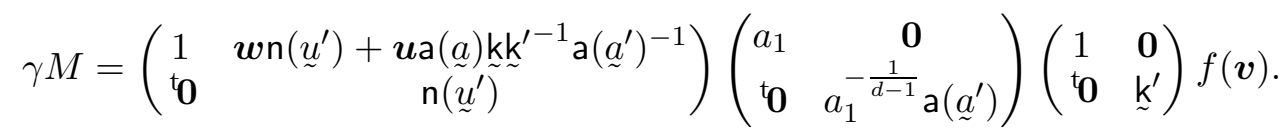

From this we see by inspection that $\gamma M \in \mathcal{G}_{A}$. Next, for each $\boldsymbol{m}=\left(m_{1}, \ldots, m_{d-1}\right) \in \mathbb{Z}^{d-1}$ with $m_{1} \neq 0$ we have $\left\|\boldsymbol{m} \gamma_{1} M\right\| \geq a_{1}^{\prime}$, since $\boldsymbol{m} \gamma_{1} \underset{\sim}{\sim} \cdot \boldsymbol{e}_{1}{\underset{\sim}{\prime}}^{\prime}=m_{1} a_{\sim}^{\prime}$. Hence any basis for the lattice $\mathbb{Z}^{d-1} \underset{\sim}{\sim}=\mathbb{Z}^{d-1} \gamma_{1} \underset{\sim}{\sim}$ must have at least one basis vector of length $\geq a_{1}^{\prime}$. But as in (3.8) we see that $\mathbb{Z}^{d-1} \underset{\sim}{\sim}$ has a basis where each basis vector has length $\leq c_{1}^{(\tilde{d}-1)}{\underset{\sim}{a}}_{1}$. Hence ${\underset{\sim}{a}}_{1}^{\prime} \leq c_{1}^{(d-1)}{\underset{\sim}{a}}_{1}=c_{1}^{(d-1)} a_{1}^{\frac{1}{d-1}} a_{2}<A a_{1}^{\frac{1}{d-1}}<a_{1}^{\frac{d}{d-1}}$. Using this fact together with $\mathrm{n}\left(\sim_{\sim}^{\prime}\right) \mathrm{a}\left(\sim_{\sim}^{\prime}\right) \underset{\sim}{\mathrm{k}}=$ $\gamma_{1} \underset{\sim}{M} \in \mathcal{F}_{d-1} \subset \mathcal{S}_{d-1}$ we see that $\gamma M \in \mathcal{S}_{d}$ (cf. (3.11) and (3.17)).

Let us define

$$
\mathcal{S}_{d}^{\prime}:=\left\{\left[a_{1}, \boldsymbol{v}, \boldsymbol{u}, \underset{\sim}{M}\right] \in \mathcal{S}_{d}: \boldsymbol{v} \in \mathrm{S}_{ \pm}^{d-1}\right\} .
$$

Then $\mathcal{S}_{d}^{\prime}$ contains a fundamental region for $\Gamma \backslash G$ (viz. $\Gamma \mathcal{S}_{d}^{\prime}=G$ ), by the argument in the beginning of the proof of Lemma 3.3. Furthermore we have $\mathcal{G}_{A} \cap \mathcal{S}_{d}=\mathcal{G}_{A} \cap \mathcal{S}_{d}^{\prime}$.

Lemma 3.4. There exists a (set-theoretical, measurable) fundamental region $\mathcal{F}_{d}$ for $\Gamma \backslash G$ which satisfies $\mathcal{F}_{d} \subset \mathcal{S}_{d}^{\prime}$ and

$$
\mathcal{G}_{A} \backslash \mathcal{C} \subset\left\{M \in \mathcal{F}_{d}: a_{1}>A\right\} \subset \mathcal{G}_{A} \cup \mathcal{C},
$$

where

$$
\begin{aligned}
\mathcal{C} & :=\left\{M \in \mathcal{S}_{d}^{\prime} \cup \mathcal{G}_{A}: a_{1}>A, a_{2} \geq\left(c_{1}^{(d-1)}\right)^{-1} A\right\} \\
& \subset\left\{\left[a_{1}, \boldsymbol{v}, \boldsymbol{u}, \underset{\sim}{M}\right] \in G: a_{1}>A, a_{2} \geq\left(c_{1}^{(d-1)}\right)^{-1} A, \boldsymbol{v} \in \mathrm{S}_{ \pm}^{d-1}, \boldsymbol{u} \in\left(-\frac{1}{2}, \frac{1}{2}\right]^{d-1}, \underset{\sim}{M} \in \mathcal{S}_{d-1}\right\} .
\end{aligned}
$$

Proof. Let $\mathcal{F}^{1}=\left\{M \in \mathcal{G}_{A}: a_{2}<\left(c_{1}^{(d-1)}\right)^{-1} A\right\}$; let $\mathcal{F}^{2}$ be an arbitrary measurable subset of $\mathcal{G}_{A} \cap \mathcal{S}_{d}^{\prime}$ which contains exactly one representative from each $\Gamma$-coset which intersects $\mathcal{G}_{A} \cap \mathcal{S}_{d}^{\prime}$ but does not intersect $\mathcal{F}^{1}$, and then let $\mathcal{F}^{3}$ be an arbitrary measurable subset of $\mathcal{S}_{d}^{\prime}$ which contains exactly one representative from each $\Gamma$-coset of $G$ which does not intersect $\mathcal{F}^{1} \cup \mathcal{F}^{2}$. $\left(\mathcal{F}^{3}\right.$ exists since $\Gamma \mathcal{S}_{d}^{\prime}=G$.) Finally set $\mathcal{F}_{d}=\mathcal{F}^{1} \cup \mathcal{F}^{2} \cup \mathcal{F}^{3}$. 
We have $\mathcal{F}^{1} \subset \mathcal{S}_{d}^{\prime}$ since $a_{2}<\left(c_{1}^{(d-1)}\right)^{-1} A \leq A<a_{1}$ implies $a_{2}<\frac{2}{\sqrt{3}} a_{1}$. Hence $\mathcal{F}_{d} \subset \mathcal{S}_{d}^{\prime}$. By Lemma 3.2 any two distinct elements in $\mathcal{F}^{1}$ are $\Gamma$-inequivalent; hence any two distinct elements in $\mathcal{F}_{d}$ are $\Gamma$-inequivalent. On the other hand we have $\Gamma \mathcal{F}_{d}=G$ by construction; hence $\mathcal{F}_{d}$ is a fundamental region for $\Gamma \backslash G$. Finally the first inclusion in (3.19) holds since $\mathcal{G}_{A} \backslash \mathcal{C} \subset \mathcal{F}^{1}$ by construction, and the second inclusion holds since $\mathcal{F}^{1} \cup \mathcal{F}^{2} \subset \mathcal{G}_{A}$ by construction and $\left\{M \in \mathcal{F}^{3}: a_{1}>A\right\} \subset \mathcal{C}$ by Lemma 3.3 .

3.3. An asymptotic formula for $\int_{\xi}^{\infty} \Phi(\eta) d \eta$. In this section we give a short proof of the following asymptotic formula.

Theorem 3.5. For any $d \geq 2$,

$$
\int_{\xi}^{\infty} \Phi(\eta) d \eta=\frac{\pi^{\frac{d-1}{2}}}{2^{d} d \Gamma\left(\frac{d+3}{2}\right) \zeta(d)} \xi^{-1}+O\left(\xi^{-1-\frac{2}{d}}\right) \quad \text { as } \xi \rightarrow \infty .
$$

Note that $\int_{\xi}^{\infty} \Phi(\eta) d \eta$ gives the limit probability that a particle in the Lorentz gas starting from a generic initial point inside the billiard domain travels length $\geq \xi$ (in macroscopic coordinates) before its first collision. In particular already Theorem 3.5 sharpens the upper bound given by Bourgain, Golse and Wennberg [6] and the lower bound of Golse and Wennberg [16]. Note that we will later prove Theorem 1.13 which gives an asymptotic formula for $\Phi(\xi)$ itself and which immediately implies Theorem 3.5. However the following short direct proof of Theorem 3.5 gives a first illustration of the usage of Lemma 3.4 in a simple case. Along the way we will prove some auxiliary results which we will need later anyway.

By definition (cf. [20, (3.8), (4.3)]),

$$
\int_{\xi}^{\infty} \Phi(\eta) d \eta=\int_{\Gamma \backslash G} \int_{[0,1)^{d} M} I\left(\left(\mathbb{Z}^{d} M+\boldsymbol{\xi}\right) \cap \mathfrak{Z}=\emptyset\right) d \boldsymbol{\xi} d \mu(M),
$$

where $\mathfrak{Z}=\mathfrak{Z}\left(0,1, \xi^{\frac{1}{d-1}}\right)$; however since the right hand side of (3.21) is invariant under $\mathfrak{Z} \mapsto \mathfrak{Z} T$ where $T$ is an arbitrary volume preserving affine linear map, we may just as well take

$$
\mathfrak{Z}=\xi^{\frac{1}{d}} \mathfrak{Z}^{\prime}, \quad \text { where } \mathfrak{Z}^{\prime}:=\mathfrak{Z}\left(-\frac{1}{2}, \frac{1}{2}, 1\right),
$$

in (3.21). Then $\mathfrak{Z}$ contains a ball of radius $\frac{1}{2} \xi^{\frac{1}{d}}$ and hence by Lemma 3.1 there is a constant $0<$ $c_{2}<1$ which only depends on $d$ such that $a_{1}>A:=c_{2} \xi^{\frac{1}{d}}$ holds for every $M=\mathrm{n}(u) \mathrm{a}(a) \mathrm{k} \in \mathcal{S}_{d}$ for which the inner integral in (3.21) is nonzero. From now on we keep $\xi$ so large that $A>1$. Fix $\mathcal{F}_{d} \subset \mathcal{S}_{d}^{\prime}$ to be a fundamental region for $\Gamma \backslash G$ as in Lemma 3.4, applied with our $A=c_{2} \xi^{\frac{1}{d}}$. Now Lemma 3.4 together with (3.12) and $a_{2}=a_{1}^{-\frac{1}{d-1}}{\underset{\sim}{a}}_{1}$ imply

$$
\begin{aligned}
\int_{\xi}^{\infty} \Phi(\eta) d \eta=\int_{\mathcal{G}_{A}} \int_{[0,1)^{d} M} I\left(\left(\mathbb{Z}^{d} M+\boldsymbol{\xi}\right) \cap \mathfrak{Z}=\emptyset\right) d \boldsymbol{\xi} d \mu(M) \\
+O\left(\int_{A}^{\infty} \mu^{(d-1)}\left(\left\{\underset{\sim}{M} \in \mathcal{S}_{d-1}:{\underset{\sim}{a}}_{1} \geq\left(c_{1}^{(d-1)}\right)^{-1} A a_{1}^{\frac{1}{d-1}}\right\}\right) \frac{d a_{1}}{a_{1}^{d+1}}\right) .
\end{aligned}
$$

If $d \geq 3$ then using (3.11) and (3.12) for $d-1$ we obtain

$$
\mu^{(d-1)}\left(\left\{\underset{\sim}{M} \in \mathcal{S}_{d-1}: a_{\sim}^{a}>T\right\}\right) \ll \int_{T}^{\infty}{\underset{\sim}{1}}_{1}^{-d} d{\underset{\sim}{a}}_{1} \ll T^{1-d}
$$

uniformly over all $T>0$. Hence the error term in (3.23) is $\ll A^{-2 d} \ll \xi^{-2}$. On the other hand if $d=2$ then the error term vanishes for all sufficiently large $\xi$.

The inner integral in the main term in (3.23) remains the same if $[0,1)^{d} M$ is replaced by any other fundamental region for $\mathbb{R}^{d} / \mathbb{Z}^{d} M$. Since $\mathbb{Z}^{d} M$ is spanned by the vectors $\boldsymbol{b}_{1}, \ldots, \boldsymbol{b}_{d}$ (cf. (3.7)), one choice of a fundamental region for $\mathbb{R}^{d} / \mathbb{Z}^{d} M$ is $\left(\prod_{j=1}^{d}\left[-\frac{a_{j}}{2}, \frac{a_{j}}{2}\right)\right) \mathrm{k}$. Hence, using 
(3.15) and (3.12), we obtain

$$
\begin{aligned}
\int_{\xi}^{\infty} \Phi(\eta) d \eta=\frac{1}{\zeta(d)} \int_{A}^{\infty} \int_{S_{1}^{\prime} d-1} \int_{\left.\left(-\frac{1}{2}, \frac{1}{2}\right]\right]^{d-1}} \int_{\mathcal{F}_{d-1}} \int_{\prod_{j=1}^{d}\left[-\frac{a_{j}}{2}, \frac{a_{j}}{2}\right)} I\left(\left(\mathbb{Z}^{d}\left[a_{1}, \boldsymbol{v}, \boldsymbol{u}, \underset{\sim}{M}\right]+\boldsymbol{w k}\right) \cap \mathfrak{Z}=\emptyset\right) \\
\times d \boldsymbol{w} d \mu(\underset{\sim}{\sim}) d \boldsymbol{u} d \boldsymbol{v} \frac{d a_{1}}{a_{1}^{d+1}}+O\left(\xi^{-2}\right),
\end{aligned}
$$

where $\mathrm{S}_{1}^{\prime d-1}:=\left\{\boldsymbol{v} \in \mathrm{S}_{1}^{d-1}: v_{1}>0\right\}$ and $\mathrm{k}=\left(\begin{array}{cc}1 & \mathbf{0} \\ \mathrm{t}_{0} & \mathrm{k}\end{array}\right) f(\boldsymbol{v})($ cf. (3.9) $)$ ).

Now for any $a_{1}, \boldsymbol{v}, \boldsymbol{u}, \underset{\sim}{M}$ and $\boldsymbol{w}=\left(w_{1}, \ldots, w_{d}\right)$ appearing in the above integral we have, by (3.14) and since $\boldsymbol{e}_{1} \mathrm{k}=\boldsymbol{v}$,

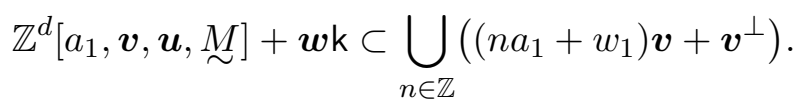

Lemma 3.6. Let $\boldsymbol{v} \in \mathrm{S}_{1}^{\prime d-1} \backslash\left\{\boldsymbol{e}_{1}\right\}$, so that the angle $\varpi$ between $\boldsymbol{v}$ and $\boldsymbol{e}_{1}$ satisfies $0<\varpi<\frac{\pi}{2}$. Set $\mathfrak{Z}^{\prime}=\mathfrak{Z}\left(-\frac{1}{2}, \frac{1}{2}, 1\right)$ as in (3.22). Then for any $t \in \mathbb{R}$, the intersection $\mathfrak{Z}^{\prime} \cap\left(t \boldsymbol{v}+\boldsymbol{v}^{\perp}\right)$ is nonempty if and only if $|t|<\frac{1}{2} \cos \varpi+\sin \varpi$, and in this case we have

$$
\begin{aligned}
\operatorname{vol}_{d-1}\left(\mathfrak{Z}^{\prime} \cap\left(t \boldsymbol{v}+\boldsymbol{v}^{\perp}\right)\right) \asymp \min \left(1, \frac{\frac{1}{2} \cos \varpi+\sin \varpi-|t|}{\sin \varpi}\right)^{\frac{d}{2}-1} \min \left(1, \frac{\frac{1}{2} \cos \varpi+\sin \varpi-|t|}{\sin 2 \varpi}\right) \\
(3.27) \\
\gg\left(\frac{1}{2} \cos \varpi+\sin \varpi-|t|\right)^{\frac{d}{2}} .
\end{aligned}
$$

Proof. After a rotation in the variables $x_{2}, \ldots, x_{d}$ we may assume $\boldsymbol{v}=\left(v_{1}, v_{2}, 0, \ldots, 0\right)$ with $v_{1}=\cos \varpi$ and $v_{2}=\sin \varpi$. By symmetry we may also assume $t \geq 0$. Now $t \boldsymbol{v}+\boldsymbol{v}^{\perp}=\left\{\boldsymbol{x} \in \mathbb{R}^{d}\right.$ : $\left.v_{1} x_{1}+v_{2} x_{2}=t\right\}$, and we see that if $\boldsymbol{x}=\left(x_{1}, \ldots, x_{d}\right)$ lies in the intersection $\mathfrak{Z}^{\prime} \cap\left(\boldsymbol{t} \boldsymbol{v}+\boldsymbol{v}^{\perp}\right)$ then so does $\left(x_{1}, x_{2}, 0, \ldots, 0\right)$. In particular $\mathfrak{Z}^{\prime} \cap\left(t \boldsymbol{v}+\boldsymbol{v}^{\perp}\right)$ is nonempty if and only if $t<\frac{1}{2} v_{1}+v_{2}$, thus proving the first assertion.

Now assume $0 \leq t<\frac{1}{2} v_{1}+v_{2}$. Let $p: \mathbb{R}^{d} \rightarrow \mathbb{R}^{d-1}$ be the projection $\left(x_{1}, \ldots, x_{d}\right) \mapsto$ $\left(x_{2}, \ldots, x_{d}\right)$, and note that

$$
p\left(\mathfrak{Z}^{\prime} \cap\left(t \boldsymbol{v}+\boldsymbol{v}^{\perp}\right)\right)=\mathcal{B}_{1}^{d-1} \cap\left((A, B) \times \mathbb{R}^{d-2}\right) \quad \text { with }\left\{\begin{array}{l}
A=\max \left(-1,\left(t-\frac{1}{2} v_{1}\right) / v_{2}\right) \\
B=\min \left(1,\left(t+\frac{1}{2} v_{1}\right) / v_{2}\right) .
\end{array}\right.
$$

Since $B>0$, it follows that

$$
\operatorname{vol}_{d-1}\left(p\left(\mathfrak{Z}^{\prime} \cap\left(t \boldsymbol{v}+\boldsymbol{v}^{\perp}\right)\right)\right) \asymp(1-A)^{\frac{d}{2}-1}(B-A) .
$$

But note that $p_{\mid t \boldsymbol{v}+\boldsymbol{v}^{\perp}}$ scales volume with a factor $v_{1}$; hence

$$
\operatorname{vol}_{d-1}\left(\mathfrak{Z}^{\prime} \cap\left(t \boldsymbol{v}+\boldsymbol{v}^{\perp}\right)\right) \asymp(1-A)^{\frac{d}{2}-1} \frac{B-A}{v_{1}} .
$$

Here $1-A=\min \left(2,\left(\frac{1}{2} v_{1}+v_{2}-t\right) / v_{2}\right)$ and

$$
\frac{B-A}{v_{1}}=\min \left(\frac{1}{v_{2}}, \frac{\frac{1}{2} v_{1}+v_{2}-t}{v_{1} v_{2}}, \frac{\frac{1}{2} v_{1}+v_{2}+t}{v_{1} v_{2}}, \frac{2}{v_{1}}\right) .
$$

Here the third entry is redundant in the minimum, since $\frac{1}{2} v_{1}+v_{2}+t \geq \frac{1}{2} v_{1}+v_{2}-t$. Also note that the minimum equals its first entry if and only if $\frac{1}{2} v_{1} \leq v_{2}-t$ and in this case we have $\frac{1}{2} v_{1} \leq v_{2}$ and thus $v_{2} \asymp 1$ (since $v_{1}=\cos \varpi, v_{2}=\sin \varpi, \varpi \in\left(0, \frac{\pi}{2}\right)$ ). Furthermore the minimum equals its fourth entry if and only if $v_{2} \leq \frac{1}{2} v_{1}-t$ and in this case we necessarily have $v_{1} \asymp 1$. Hence $\frac{B-A}{v_{1}} \asymp \min \left(1, \frac{\frac{1}{2} v_{1}+v_{2}-t}{v_{1} v_{2}}\right)$ and this concludes the proof of the first relation in (3.27). The second relation in (3.27) is obvious.

By Lemma 3.6, the cylinder $\mathfrak{Z}=\xi^{\frac{1}{d}} \mathfrak{Z}^{\prime}$ has nonempty intersection with the hyperplane $\left(n a_{1}+\right.$ $\left.w_{1}\right) \boldsymbol{v}+\boldsymbol{v}^{\perp}$ if and only if $\left|n a_{1}+w_{1}\right|<\xi^{\frac{1}{d}}\left(\frac{1}{2} \cos \varpi+\sin \varpi\right)$. It follows that if $\xi^{\frac{1}{d}}\left(\frac{1}{2} \cos \varpi+\sin \varpi\right) \leq$ $\left|w_{1}\right|<\frac{1}{2} a_{1}$ then none of the hyperplanes in (3.26) intersect $\mathfrak{Z}$, and thus $\left(\mathbb{Z}^{d}\left[\left(a_{1}, \boldsymbol{v}, \boldsymbol{u}, \mathbb{\sim}\right]+\boldsymbol{w} \mathrm{k}\right) \cap\right.$ 
$\mathfrak{Z}=\emptyset$, independently of $\boldsymbol{u}, \underset{\sim}{M}$ or $w_{2}, \ldots, w_{d}$. Hence, if we restrict the range of integration in (3.25) further by $\left|w_{1}\right| \geq \xi^{\frac{1}{d}}\left(\frac{\widetilde{1}}{2} \cos \varpi+\sin \varpi\right)$ then the resulting integral equals

$$
\begin{gathered}
\frac{1}{\zeta(d)} \int_{A}^{\infty} \int_{S_{1}^{\prime}{ }^{d-1}} \max \left(0,1-\xi^{\frac{1}{d}}(\cos \varpi+2 \sin \varpi) a_{1}^{-1}\right) d \boldsymbol{v} \frac{d a_{1}}{a_{1}^{d+1}} \\
=\frac{2 \pi^{\frac{d-1}{2}}}{\zeta(d) \Gamma\left(\frac{d-1}{2}\right)} \frac{\xi^{-1}}{d(d+1)} \int_{0}^{\pi / 2}(\cos \varpi+2 \sin \varpi)^{-d}(\sin \varpi)^{d-2} d \varpi .
\end{gathered}
$$

Here in the last step we used the fact that $\xi^{\frac{1}{d}}(\cos \varpi+2 \sin \varpi) \geq \xi^{\frac{1}{d}}>A$ for all $\varpi \in\left(0, \frac{\pi}{2}\right)$. Substituting $x=\cot \varpi$ the integral is seen to equal $2^{1-d}(d-1)^{-1}$. Hence we obtain the main term in Theorem 3.5. and to complete the proof of Theorem 3.5 we now only have to prove that, if we denote by $\mathcal{I}_{\text {rem }}$ the remaining integral, viz. the integral in (3.25) with range of integration further restricted by $\left|w_{1}\right|<\xi^{\frac{1}{d}}\left(\frac{1}{2} \cos \varpi+\sin \varpi\right)$, then

$$
\mathcal{I}_{\text {rem }}=O\left(\xi^{-1-\frac{2}{d}}\right), \quad \text { as } \xi \rightarrow \infty \text {. }
$$

To prove this, for any $a_{1}, \boldsymbol{v}, \boldsymbol{u}, \underset{\sim}{M}, \boldsymbol{w}$ appearing in the integral (3.25), we write $\boldsymbol{w}=\left(w_{1}, \boldsymbol{w}^{\prime}\right)$ with $\boldsymbol{w}^{\prime} \in \mathbb{R}^{d-1}$; then $\boldsymbol{w} \mathbf{k}=w_{1} \boldsymbol{v}+\left(0, \boldsymbol{w}^{\prime} \mathbf{k}\right) f(\boldsymbol{v})$, and hence using (3.13) we see that

$$
\mathbb{Z}^{d}\left[a_{1}, \boldsymbol{v}, \boldsymbol{u}, \underset{\sim}{M}\right]+\boldsymbol{w} \mathrm{k} \supset a_{1}^{-\frac{1}{d-1}} \iota\left(\mathbb{Z}^{d-1} \underset{\sim}{\sim}+a_{1}^{\frac{1}{d-1}} \boldsymbol{w}^{\prime} \mathbf{\sim}\right) f(\boldsymbol{v})+w_{1} \boldsymbol{v},
$$

where (here and throughout the rest of the paper) $\iota$ denotes the embedding

$$
\iota: \mathbb{R}^{d-1} \ni\left(x_{1}, \ldots, x_{d-1}\right) \mapsto\left(0, x_{1}, \ldots, x_{d-1}\right) \in \mathbb{R}^{d} .
$$

It follows that

$$
\begin{aligned}
\mathcal{I}_{\text {rem }} \ll & \int_{A}^{\infty} \int_{S_{1}^{\prime} d-1} \int_{\left|w_{1}\right|<\xi^{\frac{1}{d}}\left(\frac{1}{2} \cos \varpi+\sin \varpi\right)} \int_{\mathcal{F}_{d-1}} \int_{\boldsymbol{w}^{\prime} \in \prod_{j=2}^{d}\left[-\frac{a_{j}}{2}, \frac{a_{j}}{2}\right)} \\
(3.32) & I\left(\left(\mathbb{Z}^{d-1} \underset{\sim}{\mathcal{M}}+a_{1}^{\frac{1}{d-1}} \boldsymbol{w}^{\prime} k\right) \cap a_{1}^{\frac{1}{d-1}} \iota^{-1}\left(\left(\mathfrak{Z}-w_{1} \boldsymbol{v}\right) f(\boldsymbol{v})^{-1}\right)=\emptyset\right) d \boldsymbol{w}^{\prime} d \mu(\underset{\sim}{M}) d w_{1} d \boldsymbol{v} \frac{d a_{1}}{a_{1}^{d+1} .}
\end{aligned}
$$

Here note that $\iota^{-1}\left(\left(\mathfrak{Z}-w_{1} \boldsymbol{v}\right) f(\boldsymbol{v})^{-1}\right)$ is isometric with $\mathfrak{Z} \cap\left(w_{1} \boldsymbol{v}+\boldsymbol{v}^{\perp}\right)$, and for any $\boldsymbol{v} \in \mathrm{S}_{1}^{\prime d-1}$ with $0<\varpi<\frac{\pi}{2}$ and any $w_{1}$ with $\left|w_{1}\right|<\xi^{\frac{1}{d}}\left(\frac{1}{2} \cos \varpi+\sin \varpi\right)$, the set $\mathfrak{Z} \cap\left(w_{1} \boldsymbol{v}+\boldsymbol{v}^{\perp}\right)$ is convex and has $(d-1)$-dimensional volume $\gg \xi^{\frac{d-1}{d}}\left(\frac{1}{2} \cos \varpi+\sin \varpi-\xi^{-\frac{1}{d}}\left|w_{1}\right|\right)^{\frac{d}{2}}$, by Lemma 3.6. Hence for any $a_{1}>A$ we have

$$
\operatorname{vol}_{d-1}\left(a_{1}^{\frac{1}{d-1}} \iota^{-1}\left(\left(\mathfrak{Z}-w_{1} \boldsymbol{v}\right) f(\boldsymbol{v})^{-1}\right)\right) \gg \xi\left(\frac{1}{2} \cos \varpi+\sin \varpi-\xi^{-\frac{1}{d}}\left|w_{1}\right|\right)^{\frac{d}{2}} .
$$

Note also that $a_{1}^{\frac{1}{d-1}}\left(\prod_{j=2}^{d}\left[-\frac{a_{j}}{2}, \frac{a_{j}}{2}\right)\right) \underset{\sim}{\mathrm{k}}$ is a fundamental domain for $\mathbb{R}^{d-1} / \mathbb{Z}^{d-1} \underset{\sim}{\sim}$ for any given $a_{1}$ and $\underset{\sim}{\sim}(\mathrm{cf}$. (3.9) $)$.

Lemma 3.7. For any $d \geq 2$ and any convex subset $\subset \mathbb{R}^{d}$, we have

$$
\int_{\mathcal{F}_{d}} \int_{\mathbb{R}^{d} / \mathbb{Z}^{d} M} I\left(\left(\boldsymbol{x}+\mathbb{Z}^{d} M\right) \cap \mathfrak{C}=\emptyset\right) d \boldsymbol{x} d \mu(M) \ll \operatorname{vol}(\mathfrak{C})^{-1} .
$$

Proof. This is a straightforward modification of [29, Lemma 2.5]. In fact the bound holds for any measurable $\mathfrak{C} \subset \mathbb{R}^{d}$, as can be proved by using [1, Thm. 2.2], after first rewriting the left hand side of (3.33) as $\lim _{R \rightarrow \infty} \operatorname{vol}\left(\mathcal{B}_{R}^{d}\right)^{-1} \int_{\mathcal{B}_{R}^{d}} \int_{\mathcal{F}_{d}} I\left(\mathbb{Z}^{d} M \cap(\mathfrak{C}-\boldsymbol{x})=\emptyset\right) d \mu(M) d \boldsymbol{x}$.

If $d \geq 3$ then by Lemma 3.7 (applied with $d-1$ ) and the discussion preceding it, we obtain

$$
\begin{aligned}
\mathcal{I}_{\text {rem }} & \ll \int_{A}^{\infty} \int_{S_{1}^{\prime} d-1} \int_{\left|w_{1}\right|<\xi^{\frac{1}{d}}\left(\frac{1}{2} \cos \varpi+\sin \varpi\right)} a_{1}^{-1} \min \left\{1, \xi^{-1}\left(\frac{1}{2} \cos \varpi+\sin \varpi-\xi^{-\frac{1}{d}}\left|w_{1}\right|\right)^{-\frac{d}{2}}\right\} d w_{1} d \boldsymbol{v} \frac{d a_{1}}{a_{1}^{d+1}} \\
& \ll \xi^{-1} \int_{0}^{2} \min \left\{1, \xi^{-1} t^{-\frac{d}{2}}\right\} d t \ll \xi^{-1-\frac{2}{d}}
\end{aligned}
$$


i.e. we have proved (3.30). In the remaining case $d=2$ we have $\mathcal{F}_{d-1}=\{(1)\}=\operatorname{SL}(1, \mathbb{R})$ and hence the integrand in (3.32) vanishes whenever $\operatorname{vol}_{1}\left(a_{1} \iota^{-1}\left(\mathfrak{Z}-w_{1} \boldsymbol{v}\right) f(\boldsymbol{v})^{-1}\right)>1$; hence we obtain $\mathcal{I}_{\text {rem }} \ll \xi^{-2}$. This completes the proof of Theorem 3.5.

3.4. Asymptotics for $\Phi(\xi, \boldsymbol{w})$ : Simplifying the integral. We now start with the proof of the asymptotic formula for $\Phi(\xi, w)$, Theorem 1.11. Note that if $d=2$ then Theorem 1.11 (with $\left.F_{2}(t)=\frac{3}{2 \pi^{2}}\left((1-t)_{+}\right)^{2}\right)$ follows directly from the explicit formula in [22]. Hence we will from now on assume $d \geq 3$.

From (2.2) we have, using the right $G$-invariance of $\mu$,

$$
\Phi(\xi, w)=\int_{\Gamma \backslash G} I\left(\mathbb{Z}^{d} M \cap \mathfrak{Z}=\emptyset\right) d \mu(M),
$$

where

$$
\mathfrak{Z}:=\xi^{\frac{1}{d}}\left(\mathfrak{Z}(0,1,1)+w \boldsymbol{e}_{2}\right) .
$$

Note in particular that $\mathfrak{Z}$ does not denote the same cylinder as in the above proof of Theorem 3.5. The choice of $\mathfrak{Z}$ in (3.35) will be in force for the rest of Section 3 .

It follows from Proposition 1.10] and (1.14) (or more directly from [29, Cor. 1.4]) that there is a constant $c_{3}>0$ which only depends on $d$ such that $\Phi(\xi, w)=0$ whenever $1-w \geq c_{3} \xi^{-\frac{2}{d}}$. The function $F_{d}(t)$ appearing in the right hand side of (1.40) will be defined in (3.75) below, and it will be clear from this definition that $F_{d}(t)$ vanishes for large $t$ (cf. Lemma 3.16 or (4.14)); hence we may assume that $c_{3}$ is so large that $F_{d}(t)=0$ for all $t \geq c_{3}$. This means that (1.40) is automatic when $1-w \geq c_{3} \xi^{-\frac{2}{d}}$. Hence from now on we will assume $1-w<$ $c_{3} \xi^{-\frac{2}{d}}$. By Lemma 3.1 there is a constant $0<c_{4}<1$ which only depends on $d$ such that $a_{1}>A:=c_{4} \xi^{\frac{1}{d}}$ holds for all $M \in \mathcal{S}_{d}$ with $\mathbb{Z}^{d} M \cap \mathfrak{Z}=\emptyset$. We will assume from start that $\xi>\max \left(2,\left(10 c_{3}\right)^{d / 2}, c_{4}^{-d}\right)$; in particular we have $\frac{9}{10}<w<1$ and $A>1$.

Applying Lemma 3.4 in the same way as in (3.23) we get

$$
\begin{aligned}
& \Phi(\xi, w)=\frac{1}{\zeta(d)} \int_{S_{1}^{\prime} d-1} \int_{A}^{\infty} \int_{\left(-\frac{1}{2}, \frac{1}{2}\right]^{d-1}} \int_{\mathcal{F}_{d-1}} I\left(\mathbb{Z}^{d}\left[a_{1}, \boldsymbol{v}, \boldsymbol{u}, \underset{\sim}{M}\right] \cap \mathfrak{Z}=\emptyset\right) d \mu^{(d-1)}(\underset{\sim}{M}) d \boldsymbol{u} \frac{d a_{1}}{a_{1}^{d+1}} d \boldsymbol{v} \\
& +O\left(\xi^{-2}\right),
\end{aligned}
$$

where (again) $\mathrm{S}_{1}^{\prime d-1}:=\left\{\boldsymbol{v} \in \mathrm{S}_{1}^{d-1}: v_{1}>0\right\}$.

We parametrize a dense open subset of $\mathrm{S}_{1}^{\prime{ }^{d-1}}$ as follows (recall that we are assuming $d \geq 3$ ):

$$
\begin{aligned}
\boldsymbol{v} & =\left(v_{1}, \ldots, v_{d}\right) \\
& =\left(\cos \varpi, \sin \varpi \cos \omega,(\sin \varpi \sin \omega) \alpha_{1},(\sin \varpi \sin \omega) \alpha_{2}, \ldots,(\sin \varpi \sin \omega) \alpha_{d-2}\right) \in \mathrm{S}_{1}^{d-1},
\end{aligned}
$$

where $\varpi \in\left(0, \frac{\pi}{2}\right), \omega \in(0, \pi)$ and $\boldsymbol{\alpha}=\left(\alpha_{1}, \ldots, \alpha_{d-2}\right) \in \mathrm{S}_{1}^{d-3}$. Thus $\varpi$ is the angle between $\boldsymbol{v}$ and $\boldsymbol{e}_{1}$, and $\omega$ is the angle between $\boldsymbol{v}^{\prime}:=\left(v_{2}, \ldots, v_{d}\right)$ and $\boldsymbol{e}_{1}$ in $\mathbb{R}^{d-1}$. The $(d-1)$-dimensional volume measure on $\mathrm{S}_{1}^{d-1}$ takes the following form in our parametrization:

$$
d \boldsymbol{v}=(\sin \varpi)^{d-2}(\sin \omega)^{d-3} d \varpi d \omega d \boldsymbol{\alpha},
$$

where $d \boldsymbol{\alpha}$ is the $(d-3)$-dimensional volume measure on $\mathrm{S}_{1}^{d-3}$ (if $d=3: d \boldsymbol{\alpha}$ is the counting measure on $\left.\mathrm{S}_{1}^{0}=\{-1,1\}\right)$.

For any $\boldsymbol{v}, a_{1}, \boldsymbol{u}$ as above we have, using (3.13) with $n=0$ only:

$$
\int_{\mathcal{F}_{d-1}} I\left(\mathbb{Z}^{d}\left[a_{1}, \boldsymbol{v}, \boldsymbol{u}, \underset{\sim}{\sim}\right] \cap \mathfrak{Z}=\emptyset\right) d \mu(\underset{\sim}{M}) \leq \int_{\mathcal{F}_{d-1}} I\left(\mathbb{Z}^{d-1} \underset{\sim}{M} \cap a_{1}^{\frac{1}{d-1}} \mathfrak{Z} \boldsymbol{v}=\emptyset\right) d \mu(\underset{\sim}{M})
$$

where

$$
\mathfrak{Z}_{v}:=\iota^{-1}\left(\mathfrak{Z} f(\boldsymbol{v})^{-1}\right) \subset \mathbb{R}^{d-1}
$$


(recall the definition of $\iota$ in (3.31)). Note that $\mathfrak{Z} v$ is isometric with $\mathfrak{Z} \cap \boldsymbol{v}^{\perp}$. Now $\mathfrak{Z} \boldsymbol{v}$ contains a $(d-1)$-dimensional relatively open cone of volume $\gg \xi^{\frac{d-1}{d}} \omega^{2}(\sin \omega)^{d-2}$ with $\mathbf{0}$ in its base (cf. [29, Lemma 7.1]). Hence by [29, Cor. 1.4],

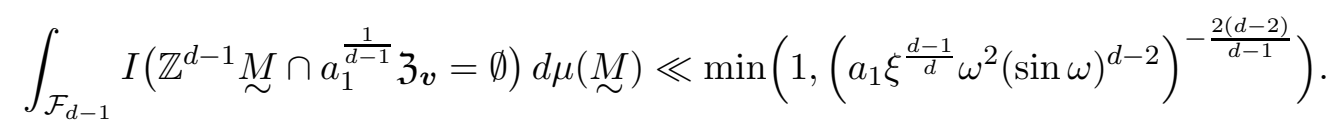

Let $0<c_{5}<1$ be an arbitrary constant. (We will later impose some conditions on $c_{5}$ being sufficiently small, but it will be clear that it is possible to fix $c_{5}$ as an absolute constant satisfying these conditions.) Now in the first line of (3.36) we may restrict the range of $\boldsymbol{v}$ to

$$
S^{\prime}:=\left\{\boldsymbol{v} \in \mathrm{S}_{1}^{\prime d-1}: 0<\omega<c_{5}\right\},
$$

at the cost of an error which, by (3.39) and (3.41), is

$$
\begin{aligned}
& \ll \int_{c_{5}}^{\pi} \int_{A}^{\infty} \min \left(1,\left(a_{1} \xi^{\frac{d-1}{d}} \omega^{2}(\sin \omega)^{d-2}\right)^{-\frac{2(d-2)}{d-1}}\right) \frac{d a_{1}}{a_{1}^{d+1}}(\sin \omega)^{d-3} d \omega \\
& \ll \xi^{-1} \int_{0}^{\pi-c_{5}} \min \left(1,\left(\xi \tau^{d-2}\right)^{-\frac{2(d-2)}{d-1}}\right) \tau^{d-3} d \tau \quad\{\text { we substituted } \tau=\pi-\omega\} \\
& \ll \xi^{-1} \int_{0}^{\xi^{-\frac{1}{d-2}}} \tau^{d-3} d \tau+\xi^{-1} \int_{\xi^{-\frac{1}{d-2}}}^{4} \xi^{-\frac{2(d-2)}{d-1}} \tau^{-1-\frac{(d-2)(d-3)}{d-1}} d \tau \ll E
\end{aligned}
$$

where we denote

$$
E:= \begin{cases}\xi^{-2} \log \xi & \text { if } d=3 \\ \xi^{-2} & \text { if } d \geq 4\end{cases}
$$

Our goal in Sec. 3.4 3.6 is to prove that (1.40) holds with the error term replaced by $O(E)$; then in Sec. 3.7 we will show how to improve the error term slightly for $d=3$ so as to complete the proof of Theorem 1.11.

Collecting our bounds so far we have, writing $M=\left[a_{1}, \boldsymbol{v}, \boldsymbol{u}, \underset{\sim}{M}\right]$,

$$
\Phi(\xi, w)=\frac{1}{\zeta(d)} \int_{S^{\prime}} \int_{A}^{\infty} \int_{\left.\left(-\frac{1}{2}, \frac{1}{2}\right]\right]^{d-1}} \int_{\mathcal{F}_{d-1}} I\left(\mathbb{Z}^{d} M \cap \mathfrak{Z}=\emptyset\right) d \mu(\underset{\sim}{M}) d \boldsymbol{u} \frac{d a_{1}}{a_{1}^{d+1}} d \boldsymbol{v}+O(E) .
$$

Lemma 3.8. For any $a_{1}>0$ and any $\boldsymbol{v}$ as in (3.37) with $\varpi \in\left(0, \frac{\pi}{2}\right)$ and $\omega \in\left[0, \frac{\pi}{2}\right]$, we have $\left(n a_{1} \boldsymbol{v}+\boldsymbol{v}^{\perp}\right) \cap \mathfrak{Z}=\emptyset$ for all $n \in \mathbb{Z} \backslash\{0\}$ if and only if $a_{1} \geq \xi^{\frac{1}{d}}\left(v_{1}+w v_{2}+\left\|\boldsymbol{v}^{\prime}\right\|\right)$.

(Recall that $\boldsymbol{v}^{\prime}:=\left(v_{2}, \ldots, v_{d}\right)$, thus $\left\|\boldsymbol{v}^{\prime}\right\|=\sin \varpi$.)

Proof. We have $\mathfrak{Z}=\xi^{\frac{1}{d}}\left(\mathfrak{Z}^{\prime}+\frac{1}{2} \boldsymbol{e}_{1}+w \boldsymbol{e}_{2}\right)$ where $\mathfrak{Z}^{\prime}=\mathfrak{Z}\left(-\frac{1}{2}, \frac{1}{2}, 1\right)$. Hence $\left(n a_{1} \boldsymbol{v}+\boldsymbol{v}^{\perp}\right) \cap \mathfrak{Z} \neq \emptyset$ if and only if $\mathfrak{Z}^{\prime}=\mathfrak{Z}\left(-\frac{1}{2}, \frac{1}{2}, 1\right)$ has nonempty intersection with

$$
\xi^{-\frac{1}{d}} n a_{1} \boldsymbol{v}-\frac{1}{2} \boldsymbol{e}_{1}-w \boldsymbol{e}_{2}+\boldsymbol{v}^{\perp}=\left(\xi^{-\frac{1}{d}} n a_{1}-\left(\frac{1}{2} v_{1}+w v_{2}\right)\right) \boldsymbol{v}+\boldsymbol{v}^{\perp} .
$$

By Lemma 3.6 this holds if and only if $\left|\xi^{-\frac{1}{d}} n a_{1}-\left(\frac{1}{2} v_{1}+w v_{2}\right)\right|<\frac{1}{2} v_{1}+\left\|\boldsymbol{v}^{\prime}\right\|$. If this inequality holds for some nonzero integer $n$ then it must hold for some positive integer $n$, since $\frac{1}{2} v_{1}+w v_{2}>$ 0 by our assumptions. But for $n>0$ we always have $\xi^{-\frac{1}{d}} n a_{1}-\left(\frac{1}{2} v_{1}+w v_{2}\right)>-\frac{1}{2} v_{1}-\left\|\boldsymbol{v}^{\prime}\right\|$. Hence we conclude that $\left(\boldsymbol{v}^{\perp}+n a_{1} \boldsymbol{v}\right) \cap \mathfrak{Z}=\emptyset$ holds for all $n \in \mathbb{Z} \backslash\{0\}$ if and only if $\xi^{-\frac{1}{d}} n a_{1}-$ $\left(\frac{1}{2} v_{1}+w v_{2}\right) \geq \frac{1}{2} v_{1}+\left\|\boldsymbol{v}^{\prime}\right\|$ for all $n \in \mathbb{Z}_{>0}$, viz. if and only if $a_{1} \geq \xi^{\frac{1}{d}}\left(v_{1}+w v_{2}+\left\|\boldsymbol{v}^{\prime}\right\|\right)$.

Lemma 3.9. For any $\boldsymbol{v}$ as in (3.37) with $\varpi \in\left(0, \frac{\pi}{2}\right)$ and $\omega \in\left[0, \frac{\pi}{2}\right]$, and any $a_{1}$ with $A=c_{4} \xi^{\frac{1}{d}}<a_{1}<\xi^{\frac{1}{d}}\left(v_{1}+w v_{2}+\left\|\boldsymbol{v}^{\prime}\right\|\right)$, we have (writing $M=\left[a_{1}, \boldsymbol{v}, \boldsymbol{u}, \underset{\sim}{\sim}\right]$ )

$$
\int_{\left(-\frac{1}{2}, \frac{1}{2}\right]^{d-1}} \int_{\mathcal{F}_{d-1}} I\left(\mathbb{Z}^{d} M \cap \mathfrak{Z}=\emptyset\right) d \mu(\underset{\sim}{M}) d \boldsymbol{u} \ll \xi^{-1}\left(v_{1}+w v_{2}+\left\|\boldsymbol{v}^{\prime}\right\|-\xi^{-\frac{1}{d}} a_{1}\right)^{-\frac{d}{2}}
$$


Proof. We use (3.13) with $n=1$ and the fact that for any $\underset{\sim}{M} \in G^{(d-1)},\left(-\frac{1}{2}, \frac{1}{2}\right]^{d-1} \mathrm{a}(\underset{\sim}{a}) \underset{\sim}{\mathrm{k}}$ is a fundamental domain for $\mathbb{R}^{d-1} / \mathbb{Z}^{d-1} \stackrel{M}{\sim}$. Writing

$$
\mathfrak{Z}_{a_{1}, \boldsymbol{v}}=\iota^{-1}\left(\left(\mathfrak{Z}-a_{1} \boldsymbol{v}\right) f(\boldsymbol{v})^{-1}\right)
$$

(a convex subset of $\mathbb{R}^{d-1}$ ) we then obtain that the left hand side of (3.46) is bounded from above by

$$
\int_{\mathcal{F}_{d-1}} \int_{\mathbb{R}^{d-1} / \mathbb{Z}^{d-1} \stackrel{M}{\sim}} I\left(\left(\boldsymbol{x}+\mathbb{Z}^{d-1} \stackrel{M}{\sim}\right) \cap a_{1}^{\frac{1}{d-1}} \mathfrak{Z}_{a_{1}, \boldsymbol{v}}=\emptyset\right) d \boldsymbol{x} d \mu(\underset{\sim}{\sim}) \ll a_{1}^{-1} \operatorname{vol}_{d-1}\left(\mathfrak{Z}_{a_{1}, \boldsymbol{v}}\right)^{-1} .
$$

Here the last inequality follows from Lemma 3.7. Now note that $\mathfrak{Z}_{a_{1}, \boldsymbol{v}}$ is isometric with $\left(a_{1} \boldsymbol{v}+\boldsymbol{v}^{\perp}\right) \cap \mathfrak{Z}$, and hence applying Lemma 3.6 (cf. the proof of Lemma 3.8) we get

$$
\begin{aligned}
\operatorname{vol}_{d-1}\left(\mathfrak{Z}_{a_{1}, \boldsymbol{v}}\right) \gg \xi^{\frac{d-1}{d}}\left(\frac{1}{2} v_{1}+\left\|\boldsymbol{v}^{\prime}\right\|\right. & \left.-\left|\xi^{-\frac{1}{d}} a_{1}-\left(\frac{1}{2} v_{1}+w v_{2}\right)\right|\right)^{\frac{d}{2}} \\
& \gg \xi^{\frac{d-1}{d}}\left(v_{1}+w v_{2}+\left\|\boldsymbol{v}^{\prime}\right\|-\xi^{-\frac{1}{d}} a_{1}\right)^{\frac{d}{2}} .
\end{aligned}
$$

Here the last relation is obvious if $\xi^{-\frac{1}{d}} a_{1} \geq \frac{1}{2} v_{1}+w v_{2}$, while if $\xi^{-\frac{1}{d}} a_{1}<\frac{1}{2} v_{1}+w v_{2}$ then it follows from

$$
\frac{1}{2} v_{1}+\left\|\boldsymbol{v}^{\prime}\right\|-\left|\xi^{-\frac{1}{d}} a_{1}-\left(\frac{1}{2} v_{1}+w v_{2}\right)\right|=\left\|\boldsymbol{v}^{\prime}\right\|-w v_{2}+\xi^{-\frac{1}{d}} a_{1}>\xi^{-\frac{1}{d}} a_{1} \gg 1 .
$$

We now obtain the stated bound.

Using (3.39), (3.41) and Lemma 3.9 it follows that the contribution from all $a_{1}$ with $a_{1}<$ $\xi^{\frac{1}{d}}\left(v_{1}+w v_{2}+\left\|\boldsymbol{v}^{\prime}\right\|\right)$ in (3.45) is, writing $t=v_{1}+w v_{2}+\left\|\boldsymbol{v}^{\prime}\right\|-\xi^{-\frac{1}{d}} a_{1}$ and using $c_{5}<1$,

$$
\begin{aligned}
& \ll \xi^{-1} \int_{0}^{1} \int_{0}^{4} \min \left\{1, \xi^{-1} t^{-\frac{d}{2}},\left(\xi \omega^{d}\right)^{-\frac{2(d-2)}{d-1}}\right\} d t \omega^{d-3} d \omega \\
& \quad \leq \xi^{-1} \int_{0}^{\xi^{-1 / d}}\left(\int_{0}^{\xi^{-2 / d}} d t+\int_{\xi^{-2 / d}}^{4} \xi^{-1} t^{-\frac{d}{2}} d t\right) \omega^{d-3} d \omega \\
& \quad+\xi^{-1} \int_{\xi^{-1 / d}}^{1}\left(\int_{0}^{\xi^{\frac{2(d-3)}{d(d-1)}} \omega^{\frac{4(d-2)}{d-1}}}\left(\xi \omega^{d}\right)^{-\frac{2(d-2)}{d-1}} d t+\int_{\xi^{\frac{2(d-3)}{d(d-1)}}}^{\infty} \omega^{\frac{4(d-2)}{d-1}} \xi^{-1} t^{-\frac{d}{2}} d t\right) \omega^{d-3} d \omega \ll E,
\end{aligned}
$$

where $E$ is as in (3.44). But for $a_{1}>\xi^{\frac{1}{d}}\left(v_{1}+w v_{2}+\left\|\boldsymbol{v}^{\prime}\right\|\right)$ we have $\mathbb{Z}^{d}\left[a_{1}, \boldsymbol{v}, \boldsymbol{u}, \underset{\sim}{M}\right] \cap \mathfrak{Z}=\emptyset$ if and only if $\mathbb{Z}^{d-1} \underset{\sim}{M} \cap a_{1}^{\frac{1}{d-1}} \mathfrak{Z}_{\boldsymbol{v}}=\emptyset$, by (3.13) , (3.14) and Lemma 3.8. Hence

$$
\Phi(\xi, w)=\frac{1}{\zeta(d)} \int_{S^{\prime}} \int_{\xi^{\frac{1}{d}}\left(v_{1}+w v_{2}+\left\|\boldsymbol{v}^{\prime}\right\|\right)}^{\infty} \int_{\mathcal{F}_{d-1}} I\left(\mathbb{Z}^{d-1} \underset{\sim}{\sim} \cap a_{1}^{\frac{1}{d-1}} \mathfrak{Z}_{\boldsymbol{v}}=\emptyset\right) d \mu(\underset{\sim}{M}) \frac{d a_{1}}{a_{1}^{d+1}} d \boldsymbol{v}+O(E) .
$$

Next we wish replace the lower integration limit for $a_{1}$ by $\xi^{\frac{1}{d}}\left(v_{1}+2 v_{2}\right)$. Note that for every $\boldsymbol{v} \in S^{\prime}$ we have

$$
\left|w v_{2}+\left\|\boldsymbol{v}^{\prime}\right\|-2 v_{2}\right| \leq 1-w+\left\|\boldsymbol{v}^{\prime}\right\|-v_{2}=1-w+(\sin \varpi)(1-\cos \omega)<1-w+\omega^{2} .
$$

Hence using (3.41) we see that the error when replacing " $\xi^{\frac{1}{d}}\left(v_{1}+w v_{2}+\left\|\boldsymbol{v}^{\prime}\right\|\right)$ " with " $\xi^{\frac{1}{d}}\left(v_{1}+\right.$ $\left.2 v_{2}\right)$ " in (3.49) is

$$
\ll \xi^{-1} \int_{0}^{1} \min \left(1,\left(\xi \omega^{d}\right)^{-\frac{2(d-2)}{d-1}}\right)\left(1-w+\omega^{2}\right)(\sin \omega)^{d-3} d \omega \ll(1-w) \xi^{-2+\frac{2}{d}}+E \ll E .
$$


(Here we used our assumption $1-w<c_{3} \xi^{-\frac{2}{d}}$.) We thus conclude:

$$
\Phi(\xi, w)=\frac{1}{\zeta(d)} \int_{S^{\prime}} \int_{\xi^{\frac{1}{d}}\left(v_{1}+2 v_{2}\right)}^{\infty} \int_{\mathcal{F}_{d-1}} I\left(\mathbb{Z}^{d-1} \stackrel{M}{\sim} \cap a_{1}^{\frac{1}{d-1}} \mathfrak{Z} \boldsymbol{v}=\emptyset\right) d \mu(\underset{\sim}{M}) \frac{d a_{1}}{a_{1}^{d+1}} d \boldsymbol{v}+O(E) .
$$

Next, for $\boldsymbol{v} \in \mathrm{S}_{1}^{\prime d-1}$ we define $L_{\boldsymbol{v}}: \mathbb{R}^{d-1} \rightarrow \mathbb{R}^{d-1}$ by

$$
L_{\boldsymbol{v}}(\boldsymbol{x})=p(\iota(\boldsymbol{x}) f(\boldsymbol{v})),
$$

where $p: \mathbb{R}^{d} \rightarrow \mathbb{R}^{d-1}$ is the projection $\left(x_{1}, \ldots, x_{d}\right) \mapsto\left(x_{2}, \ldots, x_{d}\right)$. Then $L_{\boldsymbol{v}}$ is a linear map with determinant $v_{1}>0$ (the same as the Jacobian of $p$ restricted to $\boldsymbol{v}^{\perp}$ ). Thus $v_{1}^{-\frac{1}{d-1}} L_{\boldsymbol{v}}$ is a map in $G^{(d-1)}$, and hence by the invariance of the Haar measure $\mu^{(d-1)}$ we have

$$
\int_{\mathcal{F}_{d-1}} I\left(\mathbb{Z}^{d-1} \stackrel{M}{\sim} \cap \mathfrak{C}=\emptyset\right) d \mu(\underset{\sim}{\sim})=\int_{\mathcal{F}_{d-1}} I\left(\mathbb{Z}^{d-1} \stackrel{M}{\sim} \cap v_{1}^{-\frac{1}{d-1}} L_{\boldsymbol{v}}(\mathfrak{C})=\emptyset\right) d \mu(\underset{\sim}{M})
$$

for any measurable subset $\mathfrak{C} \subset \mathbb{R}^{d-1}$. Note also that from the definition of the cylinder $\mathfrak{Z}$ it is clear that $p\left(\mathfrak{Z} \cap \boldsymbol{v}^{\perp}\right)$ is a subset of the cut ball $\xi^{\frac{1}{d}} \mathfrak{C}_{p(\boldsymbol{v})}(w)$, where

$$
\mathfrak{C}_{\boldsymbol{h}}(w)=\left\{\boldsymbol{x} \in \mathbb{R}^{d-1}:\left\|\boldsymbol{x}-w \boldsymbol{e}_{1}\right\|<1, \boldsymbol{h} \cdot \boldsymbol{x}<0\right\} .
$$

Hence

$$
L_{\boldsymbol{v}}\left(\mathfrak{Z}_{\boldsymbol{v}}\right) \subset \xi^{\frac{1}{d}} \mathfrak{C}_{p(\boldsymbol{v})}(w),
$$

and using this together with (3.53) we get

$$
\begin{aligned}
& \int_{\mathcal{F}_{d-1}} I\left(\mathbb{Z}^{d-1}\right.\left.\stackrel{M}{\sim} \cap a_{1}^{\frac{1}{d-1}} \mathfrak{Z}_{\boldsymbol{v}}=\emptyset\right) d \mu(\stackrel{M}{\sim}) \\
& \geq \mu\left(\left\{M \in X_{1}^{(d-1)}: \mathbb{Z}^{d-1} M \cap\left(\xi^{\frac{1}{d}} v_{1}^{-\frac{1}{d-1}} a_{1}^{\frac{1}{d-1}}\right) \mathfrak{C}_{p(\boldsymbol{v})}(w)=\emptyset\right\}\right) .
\end{aligned}
$$

Note that if $\boldsymbol{v}^{\perp} \cap \mathfrak{Z} \subset\left\{x_{1}<\frac{1}{2} \xi^{\frac{1}{d}}\right\}$ (say) then $p\left(\mathfrak{Z} \cap \boldsymbol{v}^{\perp}\right)$ is equal to $\xi^{\frac{1}{d}} \mathfrak{C}_{p(\boldsymbol{v})}(w)$; hence equality holds in (3.55) and hence equality also holds in (3.56). We wish to bound the contribution in (3.52) from those $\boldsymbol{v} \in S^{\prime}$ which do not satisfy this condition.

Lemma 3.10. If $\boldsymbol{v}$ satisfies $\varpi \in\left(0, \frac{\pi}{2}\right), \omega \in(0,1)$ and $\boldsymbol{v}^{\perp} \cap \mathfrak{Z} \not \subset\left\{x_{1}<\frac{1}{2} \xi^{\frac{1}{d}}\right\}$, then

$$
\frac{\pi}{2}-\varpi \ll 1-w+\omega^{2} .
$$

Proof. The assumptions imply $\boldsymbol{v}^{\perp} \cap\left(\mathfrak{Z}(0,1,1)+w \boldsymbol{e}_{2}\right) \not \subset\left\{x_{1}<\frac{1}{2}\right\}$ and thus there is some $\boldsymbol{x} \in \boldsymbol{v}^{\perp} \cap\left(\mathfrak{Z}(0,1,1)+w \boldsymbol{e}_{2}\right)$ with $x_{1} \geq \frac{1}{2}$; thus $\frac{1}{2} \leq x_{1}<1,\left\|p(\boldsymbol{x})-w \boldsymbol{e}_{1}\right\|<1$ and $\boldsymbol{v} \cdot \boldsymbol{x}=0$. Now

$$
\begin{aligned}
0< & \frac{1}{2} v_{1} \leq v_{1} x_{1}=-\left(v_{2} x_{2}+\ldots+v_{d} x_{d}\right)=-\left(v_{2}\left(x_{2}-w\right)+v_{3} x_{3}+\ldots+v_{d} x_{d}\right)-w v_{2} \\
& \leq\|p(\boldsymbol{v})\| \cdot\left\|p(\boldsymbol{x})-w \boldsymbol{e}_{1}\right\|-w v_{2} \leq\|p(\boldsymbol{v})\|-w v_{2}=(\sin \varpi)(1-w \cos \omega) \ll 1-w+\omega^{2} .
\end{aligned}
$$

This gives the desired bound, since $v_{1}=\cos \varpi \gg \frac{\pi}{2}-\varpi$.

It follows from Lemma 3.10 that the contribution in (3.52) from those $\boldsymbol{v} \in S^{\prime}$ which satisfy $\boldsymbol{v}^{\perp} \cap \mathfrak{Z} \not \subset\left\{x_{1}<\xi^{\frac{1}{d}}\right\}$ is

$$
\ll \int_{0}^{1} \int_{\substack{\frac{\pi}{2}-\varpi \in(0, \pi / 2) \\ \varpi \ll 1-w+\omega^{2}}}^{\infty} \int_{\xi^{\frac{1}{d}}\left(v_{1}+2 v_{2}\right)}^{\infty} \min \left(1,\left(a_{1} \xi^{\frac{d-1}{d}} \omega^{d}\right)^{-\frac{2(d-2)}{d-1}}\right) \frac{d a_{1}}{a_{1}^{d+1}} \varpi^{d-2} d \varpi \omega^{d-3} d \omega .
$$

This is bounded above by the expression in (3.51), and is thus $\ll E$. In view of this bound, the inequality (3.56) and the fact that equality holds in (3.56) whenever $\boldsymbol{v}^{\perp} \cap \mathfrak{Z} \subset\left\{x_{1}<\frac{1}{2} \xi^{\frac{1}{d}}\right\}$, 
we obtain:

$\Phi(\xi, w)=\frac{1}{\zeta(d)} \int_{S^{\prime}} \int_{\xi^{\frac{1}{d}}\left(v_{1}+2 v_{2}\right)}^{\infty} \mu\left(\left\{M \in X_{1}^{(d-1)}: \mathbb{Z}^{d-1} M \cap\left(\xi^{\frac{1}{d}} v_{1}^{-\frac{1}{d-1}} a_{1}^{\frac{1}{d-1}}\right) \mathfrak{C}_{p(\boldsymbol{v})}(w)=\emptyset\right\}\right) \frac{d a_{1}}{a_{1}^{d+1}} d \boldsymbol{v}$

$+O(E)$.

3.5. The paraboloid approximation. Next we will replace $\mathfrak{C}_{p(\boldsymbol{v})}(w)$ with a cut paraboloid. We first prove three easy lemmas on the approximation of a part of a unit ball with a paraboloid. (In the present section we will only need to take $u=0$ in the following, but the case of general $u$ is needed later in the treatment of $\Phi_{\mathbf{0}}(\xi, \boldsymbol{w}, \boldsymbol{z})$.)

For any $u, r$ with $|u|,|r|<1$, we let $P_{u, r} \subset \mathbb{R}^{d-1}$ be the paraboloid given by

$$
P_{u, r}:=\left\{x_{1}>A\left(x_{2}^{2}+x_{3}^{2}+\ldots+x_{d-1}^{2}\right)+B x_{2}+C\right\} ; \quad\left\{\begin{array}{l}
A=\frac{1+D}{2 \sqrt{1-u^{2}}} \\
B=-\frac{D u}{\sqrt{1-u^{2}}} \\
C=\frac{-2+(1+D) u^{2}}{2 \sqrt{1-u^{2}}}
\end{array}\right.
$$

where

$$
D=\left(\frac{u+r}{\sqrt{1-u^{2}}+\sqrt{1-r^{2}}}\right)^{2} .
$$

This definition is motivated by the following lemma, which is proved by a direct calculation.

Lemma 3.11. If $u \neq r$, then $A, B, C$ are the unique real numbers for which the parabola $x_{1}=A x_{2}^{2}+B x_{2}+C$ is tangent to the unit circle $x_{1}^{2}+x_{2}^{2}=1$ at the point $\left(-\sqrt{1-u^{2}}, u\right)$ and also intersects the circle at $\left(-\sqrt{1-r^{2}}, r\right)$.

The following lemma gives the two fundamental facts which we will use about the relation between $P_{u, r}$ and the unit ball $\mathcal{B}_{1}^{d-1}$.

\section{Lemma 3.12.}

(i) For any $0 \leq u<1$ we have $\mathcal{B}_{1}^{d-1} \subset P_{u,-u}$.

(ii) For any $0 \leq u<r<1$ we have $P_{u, r} \cap\left\{x_{1}<-\sqrt{1-r^{2}}\right\} \subset \mathcal{B}_{1}^{d-1}$.

Proof. Straightforward.

Let us write $\mathbb{R}_{+}^{d-1}$ for the upper halfspace

$$
\mathbb{R}_{+}^{d-1}:=\left\{\boldsymbol{h}=\left(h_{1}, \ldots, h_{d-1}\right) \in \mathbb{R}^{d-1}: h_{1}>0\right\} .
$$

Also, for any $\boldsymbol{h} \in \mathbb{R}^{d-1}$, we write

$$
\mathbb{R}_{\boldsymbol{h}-}^{d-1}:=\left\{\boldsymbol{x} \in \mathbb{R}^{d-1}: \boldsymbol{x} \cdot \boldsymbol{h}<0\right\} .
$$

Lemma 3.13. There is an absolute constant $c_{6}>0$ such that for any $\frac{9}{10} \leq w<1$ and $\boldsymbol{h} \in \mathbb{R}_{+}^{d-1}$, if $c_{6}\left(\sqrt{1-w}+\varphi\left(\boldsymbol{h}, \boldsymbol{e}_{1}\right)\right) \leq r \leq \frac{1}{2}$ then

$$
\left(w \boldsymbol{e}_{1}+P_{0, r}\right) \cap \mathbb{R}_{\boldsymbol{h}-}^{d-1} \subset \mathfrak{C}_{\boldsymbol{h}}(w) .
$$

Proof. We leave out the routine proof, since this lemma is a special case (obtained when $\boldsymbol{z}=\boldsymbol{w}=w \boldsymbol{e}_{1}$ ) of Lemma 4.10 which we will prove later.

With these three lemmas in place we may now approximate the integrand in (3.58) from above and below. With $c_{6}>0$ as in Lemma 3.13, set (for $\boldsymbol{v}$ as in (3.37))

$$
r=r(\boldsymbol{v})=c_{6}(\sqrt{1-w}+\omega)=c_{6}\left(\sqrt{1-w}+\varphi\left(p(\boldsymbol{v}), \boldsymbol{e}_{1}\right)\right) .
$$

From now on we assume that $\xi$ is so large that $c_{3} \xi^{-\frac{2}{d}}<\left(4 c_{6}\right)^{-2}$; thus $1-w<\left(4 c_{6}\right)^{-2}$. We also require that our constant $c_{5}$ in (3.42) should satisfy $c_{5}<\left(4 c_{6}\right)^{-1}$. Then for all $\boldsymbol{v} \in S^{\prime}$ we have $r=r(\boldsymbol{v})<\frac{1}{2}$, and hence by Lemma $3.12(\mathrm{i})$ and Lemma 3.13,

$$
\left(w \boldsymbol{e}_{1}+P_{0, r}\right) \cap \mathbb{R}_{p(\boldsymbol{v})-}^{d-1} \subset \mathfrak{C}_{p(\boldsymbol{v})}(w) \subset\left(w \boldsymbol{e}_{1}+P_{0,0}\right) \cap \mathbb{R}_{p(\boldsymbol{v})-}^{d-1} .
$$


We transform $\left(w \boldsymbol{e}_{1}+P_{0, r}\right) \cap \mathbb{R}_{p(\boldsymbol{v})-}^{d-1}$ to a "standard" cut paraboloid as follows. Set

$$
P^{d-1}:=\left\{\boldsymbol{x}=\left(x_{1}, \ldots, x_{d-1}\right) \in \mathbb{R}^{d-1}: x_{1}>x_{2}^{2}+\ldots+x_{d-1}^{2}-1\right\},
$$

and for $\boldsymbol{h} \in \mathbb{R}_{+}^{d-1}$,

$$
P_{h}^{d-1}:=P^{d-1} \cap \mathbb{R}_{h-}^{d-1} .
$$

Finally, for $\sigma \in \mathbb{R}$ and $v>0$, let $\Xi(\sigma, v)$ be the probability that a random lattice of covolume $v$ is disjoint from $P_{(1, \sigma, 0, \ldots, 0)}^{d-1}$, viz.

$$
\Xi(\sigma, v)=\mu^{(d-1)}\left(\left\{M \in X_{1}^{(d-1)}:\left(v^{\frac{1}{d-1}} \mathbb{Z}^{d-1} M\right) \cap P_{(1, \sigma, 0, \ldots, 0)}^{d-1}=\emptyset\right\}\right) .
$$

By an obvious rotational symmetry the last expression is invariant under replacement of $P_{(1, \sigma, 0, \ldots, 0)}^{d-1}$ by $P_{\boldsymbol{h}}^{d-1}$ for any $\boldsymbol{h} \in \mathbb{R}_{+}^{d-1}$ satisfying $\left\|\left(h_{2}, \ldots, h_{d-1}\right)\right\|=|\sigma| h_{1}$. Now one checks by a quick computation that if we let

$$
T=\operatorname{diag}[1-w, \underbrace{\sqrt{2 \delta(r)(1-w)}, \ldots, \sqrt{2 \delta(r)(1-w)}}_{d-2 \text { entries }}] \in \mathrm{GL}(d-1, \mathbb{R}),
$$

where

$$
\delta(r):=\frac{1}{2}\left(1+\sqrt{1-r^{2}}\right)
$$

then

$$
\left(w \boldsymbol{e}_{1}+P_{0, r}\right) \cap \mathbb{R}_{p(\boldsymbol{v})-}^{d-1}=P_{\boldsymbol{h}}^{d-1} T, \quad \text { with } \boldsymbol{h}=\left(\frac{\sqrt{1-w}}{\sqrt{2 \delta(r)}} v_{2}, v_{3}, \ldots, v_{d}\right) .
$$

This transformation formula applies also with 0 in place of $r$. Note that $T$ scales volume with a factor $2^{\frac{d}{2}-1} \delta(r)^{\frac{d}{2}-1}(1-w)^{\frac{d}{2}}$. Hence we obtain, for all $\boldsymbol{v} \in S^{\prime}$, writing $\boldsymbol{v}^{\prime \prime}=\left(v_{3}, \ldots, v_{d}\right)$,

$$
\begin{array}{r}
\Xi\left(\sqrt{\frac{2}{1-w}} \frac{\left\|\boldsymbol{v}^{\prime \prime}\right\|}{v_{2}}, \kappa \xi^{\frac{1}{d}} v_{1} a_{1}^{-1}\right) \leq \mu\left(\left\{M \in X_{1}^{(d-1)}: \mathbb{Z}^{d-1} M \cap\left(\xi^{\frac{1}{d}} v_{1}^{-\frac{1}{d-1}} a_{1}^{\frac{1}{d-1}}\right) \mathfrak{C}_{p(\boldsymbol{v})}(w)=\emptyset\right\}\right) \\
\leq \Xi\left(\sqrt{\frac{2 \delta(r)}{1-w}} \frac{\left\|\boldsymbol{v}^{\prime \prime}\right\|}{v_{2}}, \kappa \xi^{\frac{1}{d}} \delta(r)^{1-\frac{d}{2}} v_{1} a_{1}^{-1}\right),
\end{array}
$$

where

$$
\kappa=2^{1-\frac{d}{2}} \xi^{-1}(1-w)^{-\frac{d}{2}} .
$$

Using these bounds in (3.58) we conclude

$$
\Phi(\xi, w) \geq \frac{1}{\zeta(d)} \int_{S^{\prime}} \int_{\xi^{\frac{1}{d}}\left(v_{1}+2 v_{2}\right)}^{\infty} \Xi\left(\sqrt{\frac{2}{1-w}} \frac{\left\|\boldsymbol{v}^{\prime \prime}\right\|}{v_{2}}, \kappa \xi^{\frac{1}{d}} v_{1} a_{1}^{-1}\right) \frac{d a_{1}}{a_{1}^{d+1}} d \boldsymbol{v}-O(E)
$$

and

$$
\Phi(\xi, w) \leq \frac{1}{\zeta(d)} \int_{S^{\prime}} \int_{\xi^{\frac{1}{d}}\left(v_{1}+2 v_{2}\right)}^{\infty} \Xi\left(\sqrt{\frac{2 \delta(r)}{1-w}} \frac{\left\|\boldsymbol{v}^{\prime \prime}\right\|}{v_{2}}, \kappa \xi^{\frac{1}{d}} \delta(r)^{1-\frac{d}{2}} v_{1} a_{1}^{-1}\right) \frac{d a_{1}}{a_{1}^{d+1}} d \boldsymbol{v}+O(E)
$$

3.6. Further simplification of the integral; proof of Theorem 1.11 except for $d=3$. We will now simplify further the integral in (3.70). Recall that $r=r(\boldsymbol{v})=c_{6}(\sqrt{1-w}+\omega)$ in this integral. (Note that the integral in (3.69) can be viewed as a special case of the integral in (3.70), by replacing $c_{6}$ by 0 .) Substituting $a_{1}=\xi^{\frac{1}{d}} v_{1} \delta(r)^{1-\frac{d}{2}} y^{-1}$ in the inner integral, and then using the parametrization (3.37) and substituting further $\omega=\arctan \rho$ and $\varpi=\arcsin \left(v_{2} \sqrt{1+\rho^{2}}\right)$, we get

$$
\frac{\operatorname{vol}\left(\mathrm{S}_{1}^{d-3}\right) \xi^{-1}}{\zeta(d)} \int_{0}^{\tan c_{5}} \int_{0}^{\left(1+\rho^{2}\right)^{-\frac{1}{2}}}\left(\int_{0}^{\alpha \frac{v_{1}}{v_{1}+2 v_{2}}} \Xi\left(\sqrt{\frac{2 \delta(r)}{1-w}} \rho, \kappa y\right) y^{d-1} d y\right) \frac{v_{2}^{d-2}}{v_{1}^{d+1}} d v_{2} \alpha^{-d} \rho^{d-3} d \rho,
$$


where we use the notation

$$
v_{1}=\sqrt{1-v_{2}^{2}\left(1+\rho^{2}\right)}, \quad r=c_{6}(\sqrt{1-w}+\arctan \rho), \quad \alpha=\delta(r)^{1-\frac{d}{2}} .
$$

Note that $r$ and $\alpha$ only depend on the integration variable $\rho$, not on $v_{2}$ or $y$. Changing order between the two inner integrals gives

$\frac{\operatorname{vol}\left(\mathrm{S}_{1}^{d-3}\right) \xi^{-1}}{\zeta(d)} \int_{0}^{\tan c_{5}} \int_{0}^{\alpha}\left(\int_{0}^{\frac{1-y / \alpha}{\sqrt{4(y / \alpha)^{2}+\left(1+\rho^{2}\right)(1-y / \alpha)^{2}}}} \frac{v_{2}^{d-2}}{v_{1}^{d+1}} d v_{2}\right) \Xi\left(\sqrt{\frac{2 \delta(r)}{1-w}} \rho, \kappa y\right) y^{d-1} d y \alpha^{-d} \rho^{d-3} d \rho$.

Here note that for any $0 \leq x \leq\left(1+\rho^{2}\right)^{-\frac{1}{2}}$ we have

$$
\int_{0}^{x} v_{1}^{-d-1} v_{2}^{d-2} d v_{2}=\int_{0}^{x}\left(1-v_{2}^{2}\left(1+\rho^{2}\right)\right)^{-\frac{d+1}{2}} v_{2}^{d-2} d v_{2}=\frac{x^{d-1}}{(d-1)\left(1-\left(1+\rho^{2}\right) x^{2}\right)^{(d-1) / 2}},
$$

and using this with $x=\frac{1-y / \alpha}{\sqrt{4(y / \alpha)^{2}+\left(1+\rho^{2}\right)(1-y / \alpha)^{2}}}$ we conclude that the innermost integral in the above expression equals $2^{1-d}(d-1)^{-1}\left(\frac{\alpha-y}{y}\right)^{d-1}$. Hence the whole expression equals

$$
\frac{\operatorname{vol}\left(\mathrm{S}_{1}^{d-3}\right) 2^{1-d} \xi^{-1}}{(d-1) \zeta(d)} \int_{0}^{\tan c_{5}} \int_{0}^{\alpha} \Xi\left(\sqrt{\frac{2 \delta(r)}{1-w}} \rho, \kappa y\right)(\alpha-y)^{d-1} \alpha^{-d} \rho^{d-3} d y d \rho .
$$

Next we replace $\rho$ with the new variable $\sigma$ in the outer integral, via the substitution

$$
\sigma=\sqrt{\frac{2 \delta(r)}{1-w}} \rho=\sqrt{\frac{2 \delta\left(c_{6}(\sqrt{1-w}+\arctan \rho)\right)}{1-w}} \rho .
$$

Note that (using $1-w<\left(4 c_{6}\right)^{-2}$ )

$$
\frac{d \sigma}{d \rho}=\sqrt{\frac{2}{1-w}}\left(1+O\left(1-w+\rho^{2}\right)\right),
$$

uniformly over all $\rho \in\left(0, \tan \frac{1}{4 c_{6}}\right)$. Here the implied constant in the big- $O$ term is absolute (since $c_{6}$ is an absolute constant). Hence so long as $c_{5}$ is sufficiently small, our substitution is a strictly increasing (and smooth, thus with smooth inverse) map from $\rho \in\left(0, \tan c_{5}\right)$ to $\sigma \in\left(0, \sigma_{0}\right)$ where $\sigma_{0} \asymp(1-w)^{-\frac{1}{2}}$, and furthermore, for all such $\rho, \sigma$ we have

$$
\begin{aligned}
& \rho=\sqrt{\frac{1}{2}(1-w)} \sigma\left(1+O\left(1-w+\rho^{2}\right)\right)=\sqrt{\frac{1}{2}(1-w)} \sigma\left(1+O\left((1-w)\left(1+\sigma^{2}\right)\right)\right) ; \\
& \frac{d \rho}{d \sigma}=\sqrt{\frac{1}{2}(1-w)}\left(1+O\left(1-w+\rho^{2}\right)\right)=\sqrt{\frac{1}{2}(1-w)}\left(1+O\left((1-w)\left(1+\sigma^{2}\right)\right)\right) ; \\
& r=r(\sigma) \ll \sqrt{1-w}+\arctan \rho \ll \sqrt{1-w}(1+\sigma) \quad \text { and } \quad r<\frac{1}{2} .
\end{aligned}
$$

Hence our expression equals

$$
\begin{array}{r}
\frac{\operatorname{vol}\left(\mathrm{S}_{1}^{d-3}\right) 2^{2-\frac{3}{2} d}}{(d-1) \zeta(d)} \xi^{-1}(1-w)^{\frac{d}{2}-1} \int_{0}^{\sigma_{0}} \int_{0}^{\alpha} \Xi(\sigma, \kappa y) \\
\\
\times \sigma^{d-3}(1+O)^{d-1} d y \\
\end{array}
$$

Now to bound the contribution from the error term we will use the following lemma.

Lemma 3.14. For all $\sigma \geq 0$ and $v>0$ we have

$$
\Xi(\sigma, v) \ll \min \left\{1,\left((1+\sigma)^{-d} v\right)^{2-\frac{2}{d-1}}\right\} .
$$

Proof. This follows from [29, Cor. 1.4] coupled with the fact that $P_{(1, \sigma, 0, \ldots, 0)}^{d-1}$ contains a $(d-1)$ dimensional open cone of volume $\gg(1+\sigma)^{d}$ with $\mathbf{0}$ in its base. To give a slightly more detailed argument it is convenient to use a simple invariance relation which we will prove in Section 4.1; By (4.5) applied with $T=T_{\alpha, \beta}$ (cf. (4.7)), $\alpha=v^{-\frac{1}{d}}$ and $\beta=\frac{1}{2} \sigma v^{-\frac{1}{d}}$, we have

$$
\Xi(\sigma, v)=\mu\left(\left\{M \in X_{1}^{(d-1)}: \mathbb{Z}^{d-1} M \cap\left(P^{d-1}-\boldsymbol{y}\right) \cap \mathbb{R}_{\boldsymbol{e}_{1}-}^{d-1}=\emptyset\right\}\right),
$$


where

$$
\boldsymbol{y}=\left(\left(1+\frac{1}{4} \sigma^{2}\right) v^{-\frac{2}{d}}-1\right) \boldsymbol{e}_{1}+\frac{1}{2} \sigma v^{-\frac{1}{d}} \boldsymbol{e}_{2} \in P^{d-1} .
$$

Now $\left(P^{d-1}-\boldsymbol{y}\right) \cap \mathbb{R}_{\boldsymbol{e}_{1}-}^{d-1}$ contains the open cone with base $B=\left(-\frac{1}{2} \sigma v^{-\frac{1}{d}} \boldsymbol{e}_{2}+\mathcal{B}_{r}^{d-1}\right) \cap \boldsymbol{e}_{1}^{\perp}$ of radius $r=\left(1+\frac{1}{4} \sigma^{2}\right)^{\frac{1}{2}} v^{-\frac{1}{d}}$, and apex $-\boldsymbol{e}_{1}-\boldsymbol{y}$, and this cone has volume $\gg\left(1+\frac{1}{4} \sigma^{2}\right) v^{-\frac{2}{d}} r^{d-2} \gg$ $(1+\sigma)^{d} v^{-1}$. Hence the desired bound follows from [29, Cor. 1.4].

Using Lemma 3.14 we see that the contribution from the error term in (3.71) is (since $\alpha \ll 1$ over the whole range of integration)

$$
\ll \xi^{-1}(1-w)^{\frac{d}{2}} \int_{0}^{\sigma_{0}} \min \left\{1,\left(\sigma^{-d} \kappa\right)^{2-\frac{2}{d-1}}\right\} \sigma^{d-3}\left(1+\sigma^{2}\right) d \sigma .
$$

If $d \geq 4$ then this is $\ll \xi^{-2}$, even after replacing the upper integration limit $\sigma_{0}$ by $\infty$. (Here we again used our assumption $1-w<c_{3} \xi^{-\frac{2}{d}}$.) However if $d=3$ then we obtain, using the fact that $\sigma_{0} \ll(1-w)^{-\frac{1}{2}}$,

$$
\ll \xi^{-1}(1-w)^{\frac{3}{2}} \int_{0}^{\kappa^{1 / 3}}\left(1+\sigma^{2}\right) d \sigma+\xi^{-2} \int_{\kappa^{1 / 3}}^{\max \left(\kappa^{1 / 3}, \sigma_{0}\right)}\left(\sigma^{-3}+\sigma^{-1}\right) d \sigma \ll \xi^{-2} \log \xi .
$$

Hence in all cases the contribution from the error term in (3.71) is $\ll E$.

Let us also note that for any fixed $0 \leq \sigma \leq \sigma_{0}$,

$$
f(\beta)=\int_{0}^{\beta} \Xi(\sigma, \kappa y)(\beta-y)^{d-1} d y
$$

is an increasing function of $\beta \geq 1$, with derivative

$$
f^{\prime}(\beta)=(d-1) \int_{0}^{\beta} \Xi(\sigma, \kappa y)(\beta-y)^{d-2} d y .
$$

If $1 \leq \beta \ll 1$ then this derivative is $\ll \min \left\{1,\left(\sigma^{-d} \kappa\right)^{2-\frac{2}{d-1}}\right\}$, by Lemma 3.14. Since also $\alpha-1 \ll(1-w)\left(1+\sigma^{2}\right)$ we conclude that the difference caused by replacing $\alpha$ by 1 in (3.71) is bounded by exactly the same expression as in (3.73).

Hence from (3.70), we have proved that

$$
\Phi(\xi, w) \leq \frac{\operatorname{vol}\left(\mathrm{S}_{1}^{d-3}\right) 2^{2-\frac{3}{2} d}}{(d-1) \zeta(d)} \xi^{-1}(1-w)^{\frac{d}{2}-1} \int_{0}^{\infty} \int_{0}^{1} \Xi(\sigma, \kappa y)(1-y)^{d-1} \sigma^{d-3} d y d \sigma+O(E) .
$$

(We used the fact that the integrand is nonnegative to increase the $\sigma$-integration range from $\left(0, \sigma_{0}\right)$ to $(0, \infty)$.)

Finally note that the same computations allow us to compute the integral in (3.69) in exact terms; we thus obtain

$\Phi(\xi, w) \geq \frac{\operatorname{vol}\left(\mathrm{S}_{1}^{d-3}\right) 2^{2-\frac{3}{2} d}}{(d-1) \zeta(d)} \xi^{-1}(1-w)^{\frac{d}{2}-1} \int_{0}^{\sqrt{\frac{2}{1-w}} \tan c_{5}} \int_{0}^{1} \Xi(\sigma, \kappa y)(1-y)^{d-1} \sigma^{d-3} d y d \sigma-O(E)$.

By Lemma 3.14 we may here increase the upper range of $\sigma$ to $\infty$ at the cost of an error $\ll \xi^{-1}(1-w)^{\frac{d}{2}-1} \int_{\sqrt{\frac{2}{1-w}}}^{\infty} \tan c_{5} \min \left\{1, \sigma^{-d} \kappa\right\} \sigma^{d-3} d \sigma \ll \xi^{-2}(1-w)^{-1} \int_{\sqrt{\frac{2}{1-w}}}^{\infty} \tan c_{5} \sigma^{-3} d \sigma \ll \xi^{-2}$.

Hence, using $\operatorname{vol}\left(\mathrm{S}_{1}^{d-3}\right)=\frac{2 \pi^{d / 2-1}}{\Gamma(d / 2-1)}$ and $\kappa=2^{1-\frac{d}{2}} \xi^{-1}(1-w)^{-\frac{d}{2}}$, we finally obtain (1.40) with

$$
F_{d}(t)=\frac{2^{3\left(1-\frac{d}{2}\right)} \pi^{\frac{d}{2}-1}}{(d-1) \Gamma\left(\frac{d}{2}-1\right) \zeta(d)} t^{\frac{d}{2}-1} \int_{0}^{1} \int_{0}^{\infty} \Xi\left(\sigma, 2^{1-\frac{d}{2}} t^{-\frac{d}{2}} y\right) \sigma^{d-3}(1-y)^{d-1} d \sigma d y,
$$

except that we get the error term $O(E)$ (cf. (3.44)), which is slightly worse than the error term in (1.40) when $d=3$. Note that Lemma 3.14 implies that the function $F_{d}(t)$ is uniformly 
bounded over $t \in \mathbb{R}_{>0}$. Furthermore $F_{d}(t)$ is easily seen to be continuous; for instance this follows immediately from Lemma 3.16, (4.11) and Lemma 4.8 below.

Hence to complete the proof of Theorem 1.11 it now only remains to improve the error term slightly in the case $d=3$.

3.7. A slight improvement of the error term for $d=3$. The following lemma is valid for arbitrary $d \geq 3$.

Lemma 3.15. There is a constant $c_{7}>1$ which only depends on d such that if $\boldsymbol{v} \in \mathrm{S}_{1}^{\prime d-1} \backslash\left\{\boldsymbol{e}_{1}\right\}$ satisfies either $\left[\omega \leq \frac{\pi}{2}\right.$ and $\left.\omega \geq c_{7} \xi^{-1}(1-w)^{\frac{1-d}{2}}\right]$ or $\left[\omega \geq \frac{\pi}{2}\right.$ and $c_{7} \xi^{-\frac{1}{d-2}} \leq \pi-\omega \leq$ $\left.c_{7}^{-1} \xi(1-w)^{\frac{d-1}{2}}\right]$, then the left hand side of (3.41) vanishes for all $a_{1}>A=c_{4} \xi^{\frac{1}{d}}$.

Proof. For any $a_{1}>A, a_{1}^{\frac{1}{d-1}} \mathfrak{Z} v$ contains a cone of volume $\gg \xi \omega^{2}(\sin \omega)^{d-2}$ and edge ratio $\asymp \min \left(1, \frac{1-w}{\sin ^{2} \omega}\right)$ (cf. [29, Lem. 7.1]); hence by [29, Cor. 1.4], there is a constant $c>1$ such that the left hand side of (3.41) vanishes whenever $\min \left(1, \frac{1-w}{\sin ^{2} \omega}\right) \geq c\left(\xi \omega^{2}(\sin \omega)^{d-2}\right)^{-\frac{2}{d-1}}$. The lemma follows from this. (In the case $\omega \leq \frac{\pi}{2}$ one also uses $1-w<c_{3} \xi^{-\frac{2}{d}}$.)

Now if $\xi^{-1}(1-w)^{\frac{1-d}{2}}$ is sufficiently small so that $c_{7} \xi^{-1}(1-w)^{\frac{1-d}{2}} \leq c_{5}$ and $c_{7}^{-1} \xi(1-w)^{\frac{d-1}{2}}>$ $\frac{\pi}{2}$, then Lemma 3.15) says that (3.43) remains a valid bound on the error in question even if we restrict the integration range for $\tau$ to $\tau \in\left(0, c_{7} \xi^{-\frac{1}{d-2}}\right)$. This does not give any improvement if $d \geq 4$, but if $d=3$ it means that the error bound in (3.43) is improved from $\xi^{-2} \log \xi$ down to $\xi^{-2}$.

Keeping the assumption $c_{7} \xi^{-1}(1-w)^{\frac{1-d}{2}} \leq c_{5}$, Lemma 3.15 also says that we may restrict the range of $\omega$ in (3.48) to $\omega \in\left(0, c_{7} \xi^{-1}(1-w)^{\frac{1-d}{2}}\right)$. When $d=3$ this means that we improve the bound in (3.48) down to $\xi^{-2} \log \left(2+\xi^{-\frac{2}{3}}(1-w)^{-1}\right)$. In the same way also the bounds in (3.51) and (3.57) can be improved down to $\xi^{-2} \log \left(2+\xi^{-\frac{2}{3}}(1-w)^{-1}\right)$.

Finally to improve the error bounds in Section 3.6 we note the following.

Lemma 3.16. There is a constant $c_{8}>0$ which only depends on d such that $\Xi(\sigma, v)=0$ holds whenever $v \leq c_{8}(1+\sigma), \sigma \geq 0$.

Proof. This follows by noticing that the cone considered in the proof of Lemma 3.14 has edge ratio $\asymp(1+\sigma)^{-2}$, and volume $\gg(1+\sigma)^{d} v^{-1}$, and applying [29, Cor. 1.4].

Using Lemma 3.16 (and the fact that $\alpha \ll 1$ for all $\left.\sigma \in\left(0, \sigma_{0}\right)\right)$ we see that the inner integral in (3.71) vanishes unless $\sigma \ll \kappa$, and thus the bound (3.73) is valid also if we integrate over all $\sigma \ll \kappa$ instead of $\sigma \in\left(0, \sigma_{0}\right)$. Hence for $d=3$ we obtain the improved bound $\xi^{-2} \log \left(2+\kappa^{2 / 3}\right) \asymp \xi^{-2} \log \left(2+\xi^{-\frac{2}{3}}(1-w)^{-1}\right)$.

Using all these improved bounds in Sec. 3.4 3.6 we conclude that, for $d=3$, if $\xi(1-w)$ is sufficiently large so that $c_{7} \xi^{-1}(1-w)^{-1} \leq c_{5}$ and $c_{7}^{-1} \xi(1-w)>\frac{\pi}{2}$, then

$$
\Phi(\xi, w)=\xi^{-\frac{4}{3}} F_{3}\left(\xi^{\frac{2}{3}}(1-w)\right)+O\left(\xi^{-2} \log \left(2+\xi^{-\frac{2}{3}}(1-w)^{-1}\right)\right) .
$$

We have already proved in Sec. 3.6 the same formula with the error bound $\xi^{-2} \log \xi$, valid for all $w \in[0,1)$ and large $\xi$. (Recall that both $\Phi(\xi, w)$ and $\xi^{-\frac{4}{3}} F_{3}\left(\xi^{\frac{2}{3}}(1-w)\right.$ ) vanish when $1-w \geq c_{3} \xi^{-\frac{2}{3}}$.) Taking these facts together it follows that (1.40) holds. This concludes the proof of Theorem 1.11.

\section{The PARABOloid APPROXIMATION}

4.1. Definition and basic properties of the general $\Xi$-function. We now introduce a general lattice probability function $\Xi\left(\boldsymbol{y}, \boldsymbol{y}^{\prime} ; \boldsymbol{h} ; v\right)$ involving two cut paraboloids in $\mathbb{R}^{d-1}$. This is the function in terms of which we will later express our asymptotic formula for $\Phi_{\mathbf{0}}(\xi, w, z, \varphi)$ as $\xi \rightarrow \infty$, cf. Theorem 1.7 and (5.66). We keep $d \geq 3$ throughout this section. 
Recall

$$
P^{d-1}:=\left\{\boldsymbol{x}=\left(x_{1}, \ldots, x_{d-1}\right) \in \mathbb{R}^{d-1}: x_{1}>x_{2}^{2}+\ldots+x_{d-1}^{2}-1\right\},
$$

For $\boldsymbol{h} \in \mathbb{R}_{+}^{d-1}$ and $\boldsymbol{y} \in \mathbb{R}^{d-1}$ we let $P_{\boldsymbol{h}}^{d-1}(\boldsymbol{y})$ be the following cut translate of $P^{d-1}$ :

$$
P_{\boldsymbol{h}}^{d-1}(\boldsymbol{y}):=\mathbb{R}_{\boldsymbol{h}-}^{d-1} \cap\left(P^{d-1}-\boldsymbol{y}\right)=\left\{\boldsymbol{x} \in \mathbb{R}^{d-1}: \boldsymbol{x}+\boldsymbol{y} \in P^{d-1}, \boldsymbol{h} \cdot \boldsymbol{x}<0\right\} .
$$

Thus $P_{\boldsymbol{h}}^{d-1}=P_{\boldsymbol{h}}^{d-1}(\mathbf{0})$ (cf. (3.64) $)$. Now for $\boldsymbol{y}, \boldsymbol{y}^{\prime} \in \mathbb{R}^{d-1}, \boldsymbol{h} \in \mathbb{R}_{+}^{d-1}$ and $v>0$, we let $\Xi\left(\boldsymbol{y}, \boldsymbol{y}^{\prime} ; \boldsymbol{h} ; v\right)$ be the probability that a random lattice of covolume $v$ has empty intersection with both $P_{\boldsymbol{h}}^{d-1}(\boldsymbol{y})$ and $P_{\boldsymbol{h}}^{d-1}\left(\boldsymbol{y}^{\prime}\right)$, i.e.

$$
\Xi\left(\boldsymbol{y}, \boldsymbol{y}^{\prime} ; \boldsymbol{h} ; v\right):=\mu\left(\left\{M \in X_{1}^{(d-1)}:\left(v^{\frac{1}{d-1}} \mathbb{Z}^{d-1} M\right) \cap\left(P_{\boldsymbol{h}}^{d-1}(\boldsymbol{y}) \cup P_{\boldsymbol{h}}^{d-1}\left(\boldsymbol{y}^{\prime}\right)\right)=\emptyset\right\}\right) .
$$

In the special case $\boldsymbol{y}^{\prime}=\boldsymbol{y}$ we also write, for short:

$$
\Xi(\boldsymbol{y} ; \boldsymbol{h} ; v):=\Xi(\boldsymbol{y}, \boldsymbol{y} ; \boldsymbol{h} ; v) .
$$

We will in fact only consider $\Xi\left(\boldsymbol{y}, \boldsymbol{y}^{\prime} ; \boldsymbol{h} ; v\right)$ for $\boldsymbol{y}, \boldsymbol{y}^{\prime} \in \overline{P^{d-1}}$; actually we will even have $\boldsymbol{y}, \boldsymbol{y}^{\prime} \in$ $P^{d-1}$ throughout the paper except in Proposition 4.2 below.

The function $\Xi\left(\boldsymbol{y}, \boldsymbol{y}^{\prime} ; \boldsymbol{h} ; v\right)$ satisfies an invariance relation under the simultaneous transformation of the couple $\left\langle\boldsymbol{y}, \boldsymbol{y}^{\prime}\right\rangle$ by any affine linear map preserving $P^{d-1}$. Let us write $\operatorname{AGL}(d-1, \mathbb{R})$ for the group of non-singular affine linear transformations of $\mathbb{R}^{d-1}$. We represent the elements of $\operatorname{AGL}(d-1, \mathbb{R})$ by pairs $(M, \boldsymbol{\xi})$ with $M \in \operatorname{GL}(d-1, \mathbb{R})$ and $\boldsymbol{\xi} \in \mathbb{R}^{d-1}$, where the action of $(M, \boldsymbol{\xi})$ on $\mathbb{R}^{d-1}$ is given by $\boldsymbol{y} \mapsto \boldsymbol{y} M+\boldsymbol{\xi}$. Now note that for any $T=(M, \boldsymbol{\xi}) \in \operatorname{AGL}(d-1, \mathbb{R})$ which maps $P^{d-1}$ onto itself we have, directly from (4.1),

$$
P_{\boldsymbol{h}}^{d-1}(\boldsymbol{y}) M=P_{\boldsymbol{h}^{\mathrm{t}} M^{-1}}^{d-1}(\boldsymbol{y} T) .
$$

(Note here that $\boldsymbol{h}^{\mathrm{t}} M^{-1} \in \mathbb{R}_{+}^{d-1}$. Indeed, for all sufficiently large $t>0$ we have $t \boldsymbol{e}_{1} \in P^{d-1}-$ $\boldsymbol{\xi}=P^{d-1} M$ and thus $t \boldsymbol{e}_{1} M^{-1} \in P^{d-1}$. This implies $\boldsymbol{e}_{1} M^{-1}=s \boldsymbol{e}_{1}$ for some $s>0$, and hence $\boldsymbol{h}^{\mathrm{t}} M^{-1} \cdot \boldsymbol{e}_{1}=\boldsymbol{h} \cdot\left(\boldsymbol{e}_{1} M^{-1}\right)>0$.) From (4.4) and the fact that $\mu$ is preserved under $L \mapsto|\operatorname{det} M|^{-\frac{1}{d-1}} L M$, we get

$$
\Xi\left(\boldsymbol{y}, \boldsymbol{y}^{\prime} ; \boldsymbol{h} ; v\right)=\Xi\left(\boldsymbol{y} T, \boldsymbol{y}^{\prime} T ; \boldsymbol{h}^{\mathrm{t}} M^{-1} ; v|\operatorname{det} M|\right) .
$$

As a special case of this relation we have

$$
\Xi\left(\boldsymbol{y}, \boldsymbol{y}^{\prime} ; \boldsymbol{h} ; v\right)=\Xi\left(\boldsymbol{y} K, \boldsymbol{y}^{\prime} K ; \boldsymbol{h} K ; v\right) \quad \text { for any } K \in O(d-1) \text { with } \boldsymbol{e}_{1} K=\boldsymbol{e}_{1} \text {. }
$$

Next note that for any $\alpha, \beta \in \mathbb{R}, \alpha \neq 0$, the following affine linear map preserves $P^{d-1}$ :

$$
T_{\alpha, \beta}:=\left(M_{\alpha, \beta},\left(\alpha^{2}+\beta^{2}-1\right) \boldsymbol{e}_{1}+\beta \boldsymbol{e}_{2}\right), \quad M_{\alpha, \beta}=\left(\begin{array}{ccccc}
\alpha^{2} & & & & \\
2 \alpha \beta & \alpha & & & \\
& & \alpha & & \\
& & \ddots & \\
& & & \alpha
\end{array}\right) .
$$

The set of these maps $T_{\alpha, \beta}$ forms a group, with multiplication laws

$$
T_{\alpha, \beta} T_{\alpha^{\prime}, \beta^{\prime}}=T_{\alpha \alpha^{\prime}, \beta \alpha^{\prime}+\beta^{\prime}} ; \quad T_{\alpha, \beta}^{-1}=T_{\alpha^{-1},-\alpha^{-1} \beta} .
$$

Applying (4.5) repeatedly with $T=K$ as in (4.6) and $T=T_{\alpha, \beta}$ as in (4.7) we see that $\left\langle\boldsymbol{y}, \boldsymbol{y}^{\prime}\right\rangle \in$ $\left(P^{d-1}\right)^{2}$ may always be transformed to a pair of vectors with $\boldsymbol{y}=\mathbf{0}$ and $\boldsymbol{y}^{\prime} \in \operatorname{span}\left\{\boldsymbol{e}_{1}, \boldsymbol{e}_{2}\right\}$. It is now natural to define, for $a>0, b \in \mathbb{R}$,

$$
\Xi(a, b ; \boldsymbol{h} ; v):=\Xi\left(\mathbf{0}, \mathbf{0} T_{a, b} ; \boldsymbol{h} ; v\right)=\Xi\left(\mathbf{0},\left(a^{2}+b^{2}-1\right) \boldsymbol{e}_{1}+b \boldsymbol{e}_{2} ; \boldsymbol{h} ; v\right) .
$$

We note that this function satisfies the symmetry relation

$$
\Xi(a, b ; \boldsymbol{h} ; v)=\Xi\left(a^{-1}, a^{-1} b ;\left(a h_{1},-2 b h_{1}-h_{2}, h_{3}, \ldots, h_{d-1}\right) ; a^{-d} v\right) .
$$


Indeed, applying (4.5) with $T=T_{a, b}^{-1}$ we get

$$
\begin{aligned}
\Xi(a, b ; \boldsymbol{h} ; v) & =\Xi\left(\mathbf{0} T_{a, b}^{-1}, \mathbf{0} ; \boldsymbol{h}^{\mathrm{t}} M_{a, b} ; v\left|\operatorname{det} M_{a, b}^{-1}\right|\right) \\
& =\Xi\left(a^{-1},-a^{-1} b ;\left(a^{2} h_{1}, 2 a b h_{1}+a h_{2}, a h_{3}, \ldots, a h_{d-1}\right), a^{-d} v\right),
\end{aligned}
$$

and using now (4.6) with $K=\operatorname{diag}[1,-1,1, \ldots, 1]$ together with the fact that $\Xi\left(\boldsymbol{y}, \boldsymbol{y}^{\prime} ; \boldsymbol{h} ; v\right)$ only depends on the direction of $\boldsymbol{h}$ and not its length, we obtain (4.9).

Note that we are now using the same sign $\Xi$ for four different but related functions: $\Xi(\sigma, v)$, $\Xi\left(\boldsymbol{y}, \boldsymbol{y}^{\prime} ; \boldsymbol{h} ; v\right), \Xi(\boldsymbol{y} ; \boldsymbol{h} ; v)$ and $\Xi(a, b ; \boldsymbol{h} ; v)$ (cf. (3.65), (4.2), (4.3) and (4.8)). There should be no risk of confusing these, since the number or types (vector/scalar) of the arguments are different in the four cases. The relation between $\Xi(\sigma, v)$ and the other functions is of course:

$$
\Xi(\sigma, v)=\Xi(\mathbf{0} ;(1, \sigma, 0, \ldots, 0) ; v)=\Xi(1,0 ;(1, \sigma, 0, \ldots, 0) ; v) .
$$

The following lemma tells how to bring two points $\boldsymbol{y}, \boldsymbol{y}^{\prime} \in \operatorname{span}\left\{\boldsymbol{e}_{1}, \boldsymbol{e}_{2}\right\} \cap P^{d-1}$ to normal position:

Lemma 4.1. Assume that both $\boldsymbol{y}=y_{1} \boldsymbol{e}_{1}+y_{2} \boldsymbol{e}_{2}$ and $\boldsymbol{y}^{\prime}=y_{1}^{\prime} \boldsymbol{e}_{1}+y_{2}^{\prime} \boldsymbol{e}_{2}$ lie in $P^{d-1}$ (viz., $1+y_{1}-y_{2}^{2}>0$ and $\left.1+y_{1}^{\prime}-y_{2}^{\prime 2}>0\right)$. Set

$$
\alpha=\sqrt{1+y_{1}-y_{2}^{2}} ; \quad \beta=y_{2} ; \quad a=\sqrt{\frac{1+y_{1}^{\prime}-y_{2}^{\prime 2}}{1+y_{1}-y_{2}^{2}}} ; \quad b=\frac{y_{2}^{\prime}-y_{2}}{\sqrt{1+y_{1}-y_{2}^{2}}} .
$$

Then $\boldsymbol{y} T_{\alpha, \beta}^{-1}=\mathbf{0}$ and $\boldsymbol{y}^{\prime} T_{\alpha, \beta}^{-1}=\mathbf{0} T_{a, b}$.

Proof. This is verified by a direct computation.

4.2. Some properties of the function $F_{d}(t)$. In this section we prove the properties of the function $F_{d}(t)$ stated in the paragraph below Theorem 1.11, and we also derive Theorem 1.13 as a consequence of Theorem 1.11 and (1.21).

Recall that we have defined, in (3.75),

$$
F_{d}(t)=\frac{2^{3\left(1-\frac{d}{2}\right)} \pi^{\frac{d}{2}-1}}{(d-1) \Gamma\left(\frac{d}{2}-1\right) \zeta(d)} t^{\frac{d}{2}-1} \int_{0}^{1} \int_{0}^{\infty} \Xi\left(\sigma, 2^{1-\frac{d}{2}} t^{-\frac{d}{2}} y\right) \sigma^{d-3}(1-y)^{d-1} d \sigma d y .
$$

We introduce the function (for $a>0, b \in \mathbb{R}$ )

$$
\rho(a, b):=\inf \left\{v>0: \exists \boldsymbol{h} \in \mathbb{R}_{+}^{d-1}: \Xi(a, b ; \boldsymbol{h} ; v)>0\right\} .
$$

Then in particular we have (cf. (4.11))

$$
\rho(1,0)=\inf \left\{v>0: \exists \sigma \in \mathbb{R}_{\geq 0}: \Xi(\sigma ; v)>0\right\} .
$$

It follows easily from (3.65) and [29, Lemma 2.3] that $\Xi(\sigma, v)$ is a continuous function of $\sigma$ and $v$. Hence we conclude that, for $t>0$,

$$
F_{d}(t)>0 \Longleftrightarrow t<2^{\frac{2}{d}-1} \rho(1,0)^{-\frac{2}{d}}=\sqrt{\sigma_{d}(1,0)}
$$

(cf. (6.3) below for the last relation), just as stated in the introduction.

Next, it is easy to see that $F_{d}(t)$ stays bounded from below as $t \rightarrow 0$. Indeed, note that $\operatorname{vol}_{d-1}\left(v^{-\frac{1}{d-1}} P_{(1, \sigma, 0, \ldots, 0)}^{d-1}\right) \asymp(1+\sigma)^{d} v^{-1}$; hence $\Xi(\sigma, v) \gg 1$ holds whenever $(1+\sigma)^{d} v^{-1}$ is smaller than some positive constant which only depends on $d$ (cf. [29, Lemma 2.2]). In particular if $t$ is sufficiently small then the integrand in (3.75) is $\asymp \sigma^{d-3}(1-y)^{d-1}$ for all $\frac{1}{2} \leq y \leq 1, \sigma \ll t^{-\frac{1}{2}}$, and thus $F_{d}(t) \gg 1$.

However we even have that $\lim _{t \rightarrow 0} F_{d}(t)$ exists and is positive:

Proposition 4.2. For every $d \geq 3$ we have

$$
\lim _{t \rightarrow 0^{+}} F_{d}(t)=\frac{2^{3-d-\frac{2}{d}} \pi^{\frac{d}{2}-1} \Gamma(d) \Gamma\left(2-\frac{2}{d}\right)}{d(d-1) \Gamma\left(\frac{d}{2}-1\right) \Gamma\left(d+2-\frac{2}{d}\right) \zeta(d)} \int_{0}^{\infty} \Xi\left(\boldsymbol{e}_{2} ; \boldsymbol{e}_{1} ; v\right) v^{-2+\frac{2}{d}} d v .
$$


Proof. Using (4.5) with $T=T_{\alpha, \beta}, \alpha=\frac{2}{\sqrt{4+\sigma^{2}}}$ and $\beta=\frac{\sigma}{\sqrt{4+\sigma^{2}}}$ we may rewrite (3.75) as follows:

$$
F_{d}(t)=K t^{\frac{d}{2}-1} \int_{0}^{1} \int_{0}^{\infty} \Xi\left(\frac{\sigma}{\sqrt{4+\sigma^{2}}} e_{2} ; \boldsymbol{e}_{1} ; 2^{1-\frac{d}{2}} t^{-\frac{d}{2}}\left(1+\frac{1}{4} \sigma^{2}\right)^{-\frac{d}{2}} y\right) \sigma^{d-3}(1-y)^{d-1} d \sigma d y,
$$

where $K=\frac{2^{3\left(1-\frac{d}{2}\right)} \pi^{\frac{d}{2}-1}}{(d-1) \Gamma\left(\frac{d}{2}-1\right) \zeta(d)}$. We now have, uniformly over all $x \in[0,1]$ and $v>0$

$$
\begin{aligned}
& \left|\Xi\left(x \boldsymbol{e}_{2} ; \boldsymbol{e}_{1} ; v\right)-\Xi\left(\boldsymbol{e}_{2} ; \boldsymbol{e}_{1} ; v\right)\right| \\
& \leq v^{-1} \max \left\{\operatorname{vol}_{d-1}\left(P_{\boldsymbol{e}_{1}}^{d-1}\left(x \boldsymbol{e}_{2}\right) \backslash P_{\boldsymbol{e}_{1}}^{d-1}\left(\boldsymbol{e}_{2}\right)\right), \operatorname{vol}_{d_{-1}}\left(P_{\boldsymbol{e}_{1}}^{d-1}\left(\boldsymbol{e}_{2}\right) \backslash P_{\boldsymbol{e}_{1}}^{d-1}\left(x \boldsymbol{e}_{2}\right)\right)\right\} \ll v^{-1}(1-x)
\end{aligned}
$$

(cf. [29, Lemma 2.3]), and also

$$
\Xi\left(x \boldsymbol{e}_{2} ; \boldsymbol{e}_{1} ; v\right) \ll \min \left(1, v^{2-\frac{2}{d-1}}\right)
$$

(cf. [29, Cor. 1.4]). Let us keep $t<1$ for the rest of the proof. Using the above two bounds we see that the error caused by replacing $\frac{\sigma}{\sqrt{4+\sigma^{2}}} \boldsymbol{e}_{2}$ by $\boldsymbol{e}_{2}$ in (4.16) is:

$$
\begin{aligned}
\ll t^{\frac{d}{2}-1} \int_{0}^{1} \int_{0}^{\infty} \min \{1 & \left.\left(t^{-\frac{d}{2}}(1+\sigma)^{-d} y\right)^{2-\frac{2}{d-1}}, t^{\frac{d}{2}}(1+\sigma)^{d-2} y^{-1}\right\} \sigma^{d-3}(1-y)^{d-1} d \sigma d y . \\
& \ll t^{\frac{d}{2}-1} \int_{1}^{\infty} \int_{0}^{1} \min \left\{1,\left(t^{-\frac{d}{2}} \sigma^{-d}\right)^{2-\frac{2}{d-1}}, t^{\frac{d}{2}} \sigma^{d-2} y^{-1}\right\} \sigma^{d-3} d y d \sigma .
\end{aligned}
$$

To compute this we note that

$$
\int_{0}^{1} \min \left(A, B y^{-1}\right) d y= \begin{cases}B(1+\log (A / B)) & \text { if } 0<B \leq A \\ A & \text { if } 0<A \leq B .\end{cases}
$$

Furthermore note that for $1<\sigma<t^{-\frac{1}{2}}$ we have $1<\left(t^{-\frac{d}{2}} \sigma^{-d}\right)^{2-\frac{2}{d-1}}$ and $1>t^{\frac{d}{2}} \sigma^{d-2}$, while for $\sigma>t^{-\frac{1}{2}}$ we have $1>\left(t^{-\frac{d}{2}} \sigma^{-d}\right)^{2-\frac{2}{d-1}}$, and $\left(t^{-\frac{d}{2}} \sigma^{-d}\right)^{2-\frac{2}{d-1}}>t^{\frac{d}{2}} \sigma^{d-2}$ holds if and only if $\sigma<t^{c}$, where $c:=-\frac{(3 d-5) d}{2\left(3 d^{2}-7 d+2\right)}$ (note $\left.-\frac{3}{4} \leq c<-\frac{1}{2}\right)$. Writing also $c^{\prime}=\frac{3 d^{2}-7 d+2}{d-1}$ (note $c^{\prime} \geq 4$ ), we now obtain that (4.18) is

$$
\begin{aligned}
\ll t^{\frac{d}{2}-1}\left(\int_{1}^{t^{-1 / 2}} t^{\frac{d}{2}} \sigma^{2 d-5}\left(1+\log \left(t^{-\frac{d}{2}} \sigma^{2-d}\right)\right) d \sigma+\int_{t^{-1 / 2}}^{t^{c}} t^{\frac{d}{2}} \sigma^{2 d-5}\left(1+\log \left(\left(t^{c} \sigma^{-1}\right)^{c^{\prime}}\right)\right) d \sigma\right. & \\
\left.+\int_{t^{c}}^{\infty} t^{-d+\frac{d}{d-1}} \sigma^{-\frac{d^{2}-3}{d-1}} d \sigma\right) & \ll t^{\frac{d+1}{3 d-1}} .
\end{aligned}
$$

Hence we have proved

$$
F_{d}(t)=K t^{\frac{d}{2}-1} \int_{0}^{1} \int_{0}^{\infty} \Xi\left(\boldsymbol{e}_{2} ; \boldsymbol{e}_{1} ; 2^{1-\frac{d}{2}} t^{-\frac{d}{2}}\left(1+\frac{1}{4} \sigma^{2}\right)^{-\frac{d}{2}} y\right) \sigma^{d-3}(1-y)^{d-1} d \sigma d y+O\left(t^{\frac{d+1}{3 d-1}}\right) .
$$

Substituting $y=\left(1+\frac{1}{4} \sigma^{2}\right)^{\frac{d}{2}} x$ the double integral becomes:

$$
\int_{0}^{1} \Xi\left(\boldsymbol{e}_{2} ; \boldsymbol{e}_{1} ; 2^{1-\frac{d}{2}} t^{-\frac{d}{2}} x\right) \int_{0}^{2 \sqrt{x^{-2 / d}-1}}\left(1-\left(1+\frac{1}{4} \sigma^{2}\right)^{\frac{d}{2}} x\right)^{d-1}\left(1+\frac{1}{4} \sigma^{2}\right)^{\frac{d}{2}} \sigma^{d-3} d \sigma d x .
$$

Here the inner integral equals

$$
\begin{array}{r}
2^{d-3} \int_{1}^{x^{-\frac{2}{d}}}\left(1-\tau^{\frac{d}{2}} x\right)^{d-1} \tau^{\frac{d}{2}}(\tau-1)^{\frac{d}{2}-2} d \tau=2^{d-3} \int_{0}^{x^{-\frac{2}{d}}}\left(1-\tau^{\frac{d}{2}} x\right)^{d-1} \tau^{d-2} d \tau+O\left(x^{-2+\frac{4}{d}}\right) \\
=\frac{2^{d-2} \Gamma(d) \Gamma\left(2-\frac{2}{d}\right)}{d \Gamma\left(d+2-\frac{2}{d}\right)} x^{-2+\frac{2}{d}}\left(1+O\left(x^{\frac{2}{d}}\right)\right),
\end{array}
$$

uniformly over $0<x \leq 1$. Using this and the bound (4.17) we obtain (4.15) (and we furthermore see that the rate of convergence is $\left.O\left(t^{\frac{d+1}{3 d-1}}\right)\right)$. 
We next turn to the proof of Theorem 1.13 using Theorem 1.11 and (1.21). The key step in this derivation is the following formula.

\section{Proposition 4.3.}

$$
\int_{0}^{\infty} F_{d}(t) d t=\frac{2^{1-d}}{(d-1) d(d+1) \zeta(d)} .
$$

Proof of Theorem 1.13 using Proposition 4.3. We may evaluate the integral $\int_{\mathcal{B}_{1}^{d-1}} \Phi(\xi, \boldsymbol{w}) d \boldsymbol{w}$ by introducing polar coordinates $\boldsymbol{w}=(1-u) \boldsymbol{\omega}\left(0<u<1, \boldsymbol{\omega} \in \mathrm{S}_{1}^{d-2}\right)$ and using Theorem 1.11 together with the fact that $F_{d}(t)=0$ for all $t \geq \sqrt{\sigma_{d}(1,0)}$ and $\Phi(\xi, w)=0$ unless $1-w \ll \xi^{-\frac{2}{d}}$. This gives, for large $\xi$,

$$
\int_{\mathcal{B}_{1}^{d-1}} \Phi(\xi, \boldsymbol{w}) d \boldsymbol{w}=\operatorname{vol}\left(\mathrm{S}_{1}^{d-2}\right) \xi^{-2+\frac{2}{d}} \int_{0}^{1} F_{d}\left(u \xi^{\frac{2}{d}}\right)(1-u)^{d-2} d u+O\left(\xi^{-2-\frac{2}{d}}\right) .
$$

(Here for $d=3$ we used the fact that, for every fixed $c>0, \int_{0}^{c \xi^{-\frac{2}{3}}} \log \left(2+\xi^{-\frac{2}{3}} u^{-1}\right) d u \ll \xi^{-\frac{2}{3}}$.) Replacing the factor $(1-u)^{d-2}$ by 1 in the integral in the right hand side of (4.20) causes an error $\ll \xi^{-2-\frac{2}{d}}$, since $F_{d}(t)$ is uniformly bounded and of compact support. Hence we get, via Proposition 4.3,

$$
\int_{\mathcal{B}_{1}^{d-1}} \Phi(\xi, \boldsymbol{w}) d \boldsymbol{w}=\operatorname{vol}\left(\mathrm{S}_{1}^{d-2}\right) \frac{2^{1-d}}{(d-1) d(d+1) \zeta(d)} \xi^{-2}+O\left(\xi^{-2-\frac{2}{d}}\right) .
$$

This concludes the proof, in view of (1.21) and $\operatorname{vol}\left(\mathrm{S}_{1}^{d-2}\right)=2 \pi^{\frac{d-1}{2}} \Gamma\left(\frac{d-1}{2}\right)^{-1}$.

It now remains to prove Proposition 4.3, We need the following lemma.

\section{Lemma 4.4 .}

$$
\int_{P^{d-1}} \Xi\left(\boldsymbol{y} ; \boldsymbol{e}_{1} ; 1\right) d \boldsymbol{y}=1 .
$$

Proof. Let $X_{a}=X_{a}^{(d-1)} \cong \operatorname{ASL}(d-1, \mathbb{Z}) \backslash \operatorname{ASL}(d-1, \mathbb{R})$ be the space of affine lattices (i.e. translates of lattices) of covolume one in $\mathbb{R}^{d-1}$, endowed with its invariant probability measure $\mu_{a}$ and standard projection $\pi: X_{a} \rightarrow X_{1}^{(d-1)}$. Let $\widetilde{X}_{a} \subset X_{a}$ be the set of $L \in X_{a}$ for which there is a unique point $\boldsymbol{y}(L)$ which has minimal $\boldsymbol{e}_{1}$-coordinate among the points in $L \cap P^{d-1}$ (note that $\#\left(L \cap P^{d-1}\right)=\infty$ for every $\left.L \in X_{a}\right)$. Then $\widetilde{X}_{a}$ is open and of full measure in $X_{a}$, and $L \mapsto\langle\boldsymbol{y}(L), \pi(L)\rangle$ is a smooth injective map from $\widetilde{X}_{a}$ onto a certain subset $\Omega$ of $P^{d-1} \times X_{1}$, so that

$$
1=\mu_{a}\left(\widetilde{X}_{a}\right)=\int_{P^{d-1}} \int_{X_{1}} I\left(\left\langle\boldsymbol{y}, \mathbb{Z}^{d-1} M\right\rangle \in \Omega\right) d \mu(M) d \boldsymbol{y} .
$$

Now for given $\boldsymbol{y} \in P^{d-1}$ and $M \in X_{1}$ we have $\left\langle\boldsymbol{y}, \mathbb{Z}^{d-1} M\right\rangle \in \Omega$ if and only if $\mathbb{Z}^{d-1} M \cap$ $P_{\boldsymbol{e}_{1}}^{d-1}(\boldsymbol{y})=\emptyset$ and $\mathbb{Z}^{d-1} M \cap\left(P^{d-1}-\boldsymbol{y}\right) \cap\left\{x_{1}=0\right\}=\{\mathbf{0}\}$. The last condition holds for $\mu-$ almost all $M \in X_{1}$. Hence the inner integral in (4.22) equals $\Xi\left(\boldsymbol{y} ; \boldsymbol{e}_{1} ; 1\right)$, and this proves the lemma.

Proof of Proposition 4.3. Using (4.5) with $T=T_{v^{-\frac{1}{d}}, \frac{1}{2} \sigma v^{-\frac{1}{d}}}$, where $v=2^{1-\frac{d}{2}} t^{-\frac{d}{2}} y$ we get, for any $t, \sigma>0,0<y<1$,

$$
\Xi\left(\sigma, 2^{1-\frac{d}{2}} t^{-\frac{d}{2}} y\right)=\Xi\left(a \boldsymbol{e}_{1}+b \boldsymbol{e}_{2} ; \boldsymbol{e}_{1} ; 1\right)
$$

where (for any fixed $t>0$ )

$$
\left\{\begin{array}{l}
a=a(\sigma, y)=\left(\frac{1}{4} \sigma^{2}+1\right)\left(2^{1-\frac{2}{d}} t y^{-\frac{2}{d}}\right)-1 \\
b=b(\sigma, y)=2^{-\frac{1}{2}-\frac{1}{d}} \sigma t^{\frac{1}{2}} y^{-\frac{1}{d}}
\end{array}\right.
$$


One checks that the map $\langle\sigma, y\rangle \mapsto\langle a, b\rangle$ is a diffeomorphism from $(0, \infty) \times(0,1)$ onto $\{\langle a, b\rangle$ : $\left.b>0, a>b^{2}-1+2^{1-\frac{2}{d}} t\right\}$, with the inverse map

$$
\left\{\begin{array}{l}
\sigma=2 b\left(a+1-b^{2}\right)^{-\frac{1}{2}} \\
y=2^{\frac{d}{2}-1} t^{\frac{d}{2}}\left(a+1-b^{2}\right)^{-\frac{d}{2}}
\end{array}\right.
$$

and the Jacobian is

$$
\left|\frac{\partial(\sigma, y)}{\partial(a, b)}\right|=2^{\frac{d}{2}-1} d t^{\frac{d}{2}}\left(a+1-b^{2}\right)^{-\frac{d+3}{2}} .
$$

Hence (3.75) may be rewritten as follows:

$$
\begin{aligned}
F_{d}(t)=\frac{2^{-1} d \pi^{\frac{d}{2}-1}}{(d-1) \Gamma\left(\frac{d}{2}-1\right) \zeta(d)} t^{d-1} \int_{0}^{\infty} \int_{b^{2}-1+2^{1-\frac{2}{d} t}}^{\infty} \Xi\left(a \boldsymbol{e}_{1}+b \boldsymbol{e}_{2} ; \boldsymbol{e}_{1} ; 1\right) \\
\times\left(a+1-b^{2}\right)^{-d} b^{d-3}\left(1-2^{\frac{d}{2}-1} t^{\frac{d}{2}}\left(a+1-b^{2}\right)^{-\frac{d}{2}}\right)^{d-1} d a d b .
\end{aligned}
$$

Substituting this formula in $\int_{0}^{\infty} F_{d}(t) d t$ and then changing the order of integration and using

$$
\int_{0}^{2^{\frac{2}{d-1}\left(a+1-b^{2}\right)}} t^{d-1}\left(1-2^{\frac{d}{2}-1} t^{\frac{d}{2}}\left(a+1-b^{2}\right)^{-\frac{d}{2}}\right)^{d-1} d t=\frac{2^{3-d}\left(a+1-b^{2}\right)^{d}}{d^{2}(d+1)}
$$

we get

$$
\int_{0}^{\infty} F_{d}(t) d t=\frac{2^{2-d} \pi^{\frac{d}{2}-1}}{(d-1) d(d+1) \Gamma\left(\frac{d}{2}-1\right) \zeta(d)} \int_{0}^{\infty} \int_{b^{2}-1}^{\infty} \Xi\left(a \boldsymbol{e}_{1}+b \boldsymbol{e}_{2} ; \boldsymbol{e}_{1} ; 1\right) b^{d-3} d a d b
$$

Now use the fact that $\Xi\left(a \boldsymbol{e}_{1}+b \boldsymbol{e}_{2} ; \boldsymbol{e}_{1} ; 1\right)=\Xi\left(a \boldsymbol{e}_{1}+b \boldsymbol{\omega} ; \boldsymbol{e}_{1} ; 1\right)$ for any $\boldsymbol{\omega} \in\{0\} \times \mathrm{S}_{1}^{d-3} \subset \mathbb{R}^{d-1}$ and integrate over this sphere; this gives

$$
\int_{0}^{\infty} F_{d}(t) d t=\frac{2^{1-d}}{(d-1) d(d+1) \zeta(d)} \int_{P^{d-1}} \Xi\left(\boldsymbol{y} ; \boldsymbol{e}_{1} ; 1\right) d \boldsymbol{y},
$$

and now (4.19) is a consequence of Lemma 4.4.

\subsection{On the size and continuity of $\Xi(a, b ; \boldsymbol{h} ; v)$.}

Lemma 4.5. For all $a>0, b \in \mathbb{R}, \boldsymbol{h}=\left(h_{1}, \ldots, h_{d-1}\right) \in \mathbb{R}_{+}^{d-1}$ and $v>0$ we have

$$
\begin{aligned}
\Xi(a, b ; \boldsymbol{h} ; v) \ll \min \left\{1,\left(\left(1+\frac{\left\|\boldsymbol{h}^{\prime}\right\|}{h_{1}}\right)^{-d} v\right)^{2-\frac{2}{d-1}},\right. \\
\left.\quad\left(\left(a+\frac{\left\|\left(2 b h_{1}+h_{2}, h_{3}, \ldots, h_{d-1}\right)\right\|}{h_{1}}\right)^{-d} v\right)^{2-\frac{2}{d-1}}\right\},
\end{aligned}
$$

where $\boldsymbol{h}^{\prime}=\left(h_{2}, \ldots, h_{d-1}\right)$.

Proof. It is clear from the definitions and (4.6) that $\Xi(a, b ; \boldsymbol{h} ; v) \leq \Xi(\mathbf{0} ; \boldsymbol{h} ; v)=\Xi\left(\frac{\left\|\boldsymbol{h}^{\prime}\right\|}{h_{1}}, v\right)$; hence by Lemma 3.14 we have

$$
\Xi(a, b ; \boldsymbol{h} ; v) \ll \min \left\{1,\left(\left(1+\frac{\left\|\boldsymbol{h}^{\prime}\right\|}{h_{1}}\right)^{-d} v\right)^{2-\frac{2}{d-1}}\right\} .
$$

The lemma follows from this bound by also using (4.9).

Lemma 4.6. For any $x, y>0$ and $\alpha \geq \max \left(\frac{y}{x}, 1\right)$ we have

$$
P^{d-1}-\left(y e_{1}+e_{2}\right) \subset \alpha\left(P^{d-1}-\left(x e_{1}+e_{2}\right)\right) .
$$


Proof. The point $\boldsymbol{x}=\left(x_{1}, \ldots, x_{d-1}\right)$ belongs to the set in the left hand side of (4.30) if and only if $x_{1}>\left(x_{2}+1\right)^{2}+x_{3}^{2}+\ldots+x_{d-1}^{2}-(1+y)$, and it belongs to the set in the right hand side of (4.30) if and only if $\frac{x_{1}}{\alpha}>\left(\frac{x_{2}}{\alpha}+1\right)^{2}+\left(\frac{x_{3}}{\alpha}\right)^{2}+\ldots+\left(\frac{x_{d-1}}{\alpha}\right)^{2}-(1+x)$. Hence our task is to prove

$$
\alpha\left(\left(x_{2}+1\right)^{2}+x_{3}^{2}+\ldots+x_{d-1}^{2}-(1+y)\right) \geq\left(x_{2}+\alpha\right)^{2}+x_{3}^{2}+\ldots+x_{d-1}^{2}-\alpha^{2}(1+x),
$$

for all $x_{2}, \ldots, x_{d-1} \in \mathbb{R}$. This simplifies to

$$
(\alpha-1)\left(x_{2}^{2}+x_{3}^{2}+\ldots+x_{d-1}^{2}\right)+\alpha(\alpha x-y) \geq 0,
$$

which is clear when $\alpha \geq \max \left(\frac{y}{x}, 1\right)$.

Lemma 4.7. For any fixed $a, b, \boldsymbol{h}, \Xi(a, b ; \boldsymbol{h} ; v)$ is an increasing function of $v \in \mathbb{R}_{>0}$.

Proof. It suffices that $\Xi\left(\boldsymbol{y}, \boldsymbol{y}^{\prime} ; \boldsymbol{h} ; v\right)$ is an increasing function of $v$ for fixed $\boldsymbol{y}, \boldsymbol{y}^{\prime} \in P^{d-1}, \boldsymbol{h} \in$ $\mathbb{R}_{+}^{d-1}$. This follows directly from the definition (4.2), since

$$
v^{-\frac{1}{d-1}}\left(P_{\boldsymbol{h}}^{d-1}(\boldsymbol{y}) \cup P_{\boldsymbol{h}}^{d-1}\left(\boldsymbol{y}^{\prime}\right)\right) \subset v^{\prime-\frac{1}{d-1}}\left(P_{\boldsymbol{h}}^{d-1}(\boldsymbol{y}) \cup P_{\boldsymbol{h}}^{d-1}\left(\boldsymbol{y}^{\prime}\right)\right)
$$

whenever $v^{\prime} \leq v$.

Lemma 4.8. $\Xi(a, b ; \boldsymbol{h} ; v)$ is a continuous function on $\mathbb{R}_{>0} \times \mathbb{R} \times \mathbb{R}_{+}^{d-1} \times \mathbb{R}_{>0}$.

Proof. It suffices to prove that $\Xi\left(\boldsymbol{y}, \boldsymbol{y}^{\prime} ; \boldsymbol{h} ; v\right)$ is continuous. Fix $\left\langle\boldsymbol{y}, \boldsymbol{y}^{\prime}, \boldsymbol{h}, v\right\rangle \in P^{d-1} \times P^{d-1} \times$ $\mathbb{R}_{+}^{d-1} \times \mathbb{R}_{>0}$ and let $\left\langle\tilde{\boldsymbol{y}}, \tilde{\boldsymbol{y}}^{\prime}, \tilde{\boldsymbol{h}}, \tilde{v}\right\rangle$ run through a sequence of tuples in $P^{d-1} \times P^{d-1} \times \mathbb{R}_{+}^{d-1} \times \mathbb{R}_{>0}$ tending to $\left\langle\boldsymbol{y}, \boldsymbol{y}^{\prime}, \boldsymbol{h}, v\right\rangle$. Then

$$
\begin{aligned}
\limsup _{\left\langle\tilde{\boldsymbol{y}}, \tilde{\boldsymbol{y}}^{\prime}, \tilde{\boldsymbol{h}}, \tilde{v}\right\rangle}\left(v ^ { - \frac { 1 } { d - 1 } } ( P _ { \boldsymbol { h } } ^ { d - 1 } ( \boldsymbol { y } ) \cup P _ { \boldsymbol { h } } ^ { d - 1 } ( \boldsymbol { y } ^ { \prime } ) ) \triangle \tilde { v } ^ { - \frac { 1 } { d - 1 } } \left(P_{\tilde{\boldsymbol{h}}}^{d-1}(\tilde{\boldsymbol{y}}) \cup\right.\right. & \left.\left.P_{\tilde{\boldsymbol{h}}}^{d-1}\left(\tilde{\boldsymbol{y}}^{\prime}\right)\right)\right) \\
& \subset v^{-\frac{1}{d-1}} \partial\left(P_{\boldsymbol{h}}^{d-1}(\boldsymbol{y}) \cup P_{\boldsymbol{h}}^{d-1}\left(\boldsymbol{y}^{\prime}\right)\right),
\end{aligned}
$$

and thus ([14, Thm. 1.2.2])

$$
\limsup _{\left\langle\tilde{\boldsymbol{y}}, \tilde{\boldsymbol{y}}^{\prime}, \tilde{\boldsymbol{h}}, \tilde{v}\right\rangle} \operatorname{vol}_{d-1}\left(v^{-\frac{1}{d-1}}\left(P_{\boldsymbol{h}}^{d-1}(\boldsymbol{y}) \cup P_{\boldsymbol{h}}^{d-1}\left(\boldsymbol{y}^{\prime}\right)\right) \triangle \tilde{v}^{-\frac{1}{d-1}}\left(P_{\tilde{\boldsymbol{h}}}^{d-1}(\tilde{\boldsymbol{y}}) \cup P_{\tilde{\boldsymbol{h}}}^{d-1}\left(\tilde{\boldsymbol{y}}^{\prime}\right)\right)\right)=0 .
$$

Hence by [29, Lemma 2.3],

$$
\limsup _{\left\langle\tilde{\boldsymbol{y}}, \tilde{\boldsymbol{y}}^{\prime}, \tilde{\boldsymbol{h}}, \tilde{v}\right\rangle}\left|\Xi\left(\tilde{\boldsymbol{y}}, \tilde{\boldsymbol{y}}^{\prime} ; \tilde{\boldsymbol{h}} ; \tilde{v}\right)-\Xi\left(\boldsymbol{y}, \boldsymbol{y}^{\prime} ; \boldsymbol{h} ; v\right)\right|=0 .
$$

Lemma 4.9. There is a constant $c>0$ which only depends on $d$ such that, for all $0<a \leq 10$, $b \in \mathbb{R}, v>0, \boldsymbol{h}=\left(h_{1}, \ldots, h_{d-1}\right) \in \mathbb{R}_{+}^{d-1}$ and $\tau_{a}, \tau_{b} \in\left[\frac{1}{2}, \frac{3}{2}\right]$, we have

$$
\Xi(a, b ; \boldsymbol{h} ; v) \leq \Xi\left(\tau_{a} a, \tau_{b} b ;\left(\tau_{b}^{-1} h_{1}, h_{2}, \ldots, h_{d-1}\right) ;\left(1+c\left(\left|\tau_{a}-1\right|+\left|\tau_{b}-1\right|\right)\right) v\right) .
$$

Proof. Set $a^{\prime}=\tau_{a} a, b^{\prime}=\tau_{b} b$ and $\boldsymbol{k}=\left(\tau_{b}^{-1} h_{1}, h_{2}, \ldots, h_{d-1}\right)$. It suffices to prove the inequality for $b \neq 0$ since the case $b=0$ then follows by continuity (Lemma 4.8). By (4.5) with $T=T_{-2 / b^{\prime}, 1}$ we have, for any $v^{\prime}>0$,

$$
\Xi\left(a^{\prime}, b^{\prime} ; \boldsymbol{k} ; v^{\prime}\right)=\mu\left(\left\{M \in X_{1}: \mathbb{Z}^{d-1} M \cap w^{\prime}\left(P_{\ell}^{d-1}\left(\boldsymbol{y}^{\prime}\right) \cup P_{\ell}^{d-1}\left(\boldsymbol{z}^{\prime}\right)\right)=\emptyset\right\}\right)
$$

where $\boldsymbol{\ell}=\boldsymbol{k}^{\mathrm{t}} M_{-2 / b^{\prime}, 1}^{-1}, \boldsymbol{y}^{\prime}=\frac{4}{b^{\prime 2}} \boldsymbol{e}_{1}+\boldsymbol{e}_{2}, \boldsymbol{z}^{\prime}=\frac{4 a^{2}}{{b^{\prime 2}}^{2}} \boldsymbol{e}_{1}-\boldsymbol{e}_{2}$ and $w^{\prime}=2^{-\frac{d}{d-1}}\left|b^{\prime}\right|^{\frac{d}{d-1}} v^{-\frac{1}{d-1}}$. Similarly, using the fact that $\boldsymbol{h}^{\mathrm{t}} M_{-2 / b, 1}^{-1} \sim \boldsymbol{\ell}$,

$$
\Xi(a, b ; \boldsymbol{h} ; v)=\mu\left(\left\{M \in X_{1}: \mathbb{Z}^{d-1} M \cap w\left(P_{\ell}^{d-1}(\boldsymbol{y}) \cup P_{\ell}^{d-1}(\boldsymbol{z})\right)=\emptyset\right\}\right)
$$


where $\boldsymbol{y}=\frac{4}{b^{2}} \boldsymbol{e}_{1}+\boldsymbol{e}_{2}, \boldsymbol{z}=\frac{4 a^{2}}{b^{2}} \boldsymbol{e}_{1}-\boldsymbol{e}_{2}$ and $w=2^{-\frac{d}{d-1}}|b|^{\frac{d}{d-1}} v^{-\frac{1}{d-1}}$. Now take $\delta=\max \left(1, \tau_{b}^{-2}, \tau_{a}^{2} \tau_{b}^{-2}\right)$; then by Lemma 4.6 we have

$$
w^{\prime} P_{\ell}^{d-1}\left(\boldsymbol{y}^{\prime}\right) \subset \delta w^{\prime} P_{\ell}^{d-1}(\boldsymbol{y}) \quad \text { and } \quad w^{\prime} P_{\ell}^{d-1}\left(\boldsymbol{z}^{\prime}\right) \subset \delta w^{\prime} P_{\ell}^{d-1}(\boldsymbol{z}),
$$

and thus $\Xi(a, b ; \boldsymbol{h} ; v) \leq \Xi\left(a^{\prime}, b^{\prime} ; \boldsymbol{k} ; v^{\prime}\right)$ holds so long as $w \geq \delta w^{\prime}$, viz. $v \leq \delta^{1-d} \tau_{b}^{-d} v^{\prime}$, which is certainly true for $v^{\prime}=\left(1+c\left(\left|\tau_{a}-1\right|+\left|\tau_{b}-1\right|\right)\right) v$ with $c$ sufficiently large.

4.4. Approximating packing probabilites with $\Xi(a, b ; \boldsymbol{h} ; v)$. Just as in the proof of Theorem 1.11 a crucial step was to approximate the probability of a random lattice avoiding a given cut ball by the corresponding probability for a cut paraboloid (cf. Sec. 3.5), so it will be important in the proof of Theorem 1.7 to carry out the corresponding approximation for a union of two given cut balls. Given $\boldsymbol{h} \in \mathbb{R}^{d-1} \backslash\{\mathbf{0}\}$ and $\boldsymbol{w}, \boldsymbol{z} \in \mathcal{B}_{1}^{d-1}$ we define

$$
\mathfrak{C}_{\boldsymbol{h}}(\boldsymbol{w}):=\left(\boldsymbol{w}+\mathcal{B}_{1}^{d-1}\right) \cap \mathbb{R}_{h-}^{d-1}
$$

(this agrees with (3.54) if $\boldsymbol{w}=w \boldsymbol{e}_{1}$ ) and set

$$
\mathfrak{C}_{\boldsymbol{h}}(\boldsymbol{z}, \boldsymbol{w}):=\mathfrak{C}_{\boldsymbol{h}}(\boldsymbol{z}) \cup \mathfrak{C}_{\boldsymbol{h}}(\boldsymbol{w})
$$

Finally let $\Upsilon(\boldsymbol{z}, \boldsymbol{w}, \boldsymbol{h}, v)$ be the probability that a random lattice of covolume $v$ has empty intersection with $\mathfrak{C}_{\boldsymbol{h}}(\boldsymbol{z}, \boldsymbol{w})$, viz.

$$
\Upsilon(\boldsymbol{z}, \boldsymbol{w}, \boldsymbol{h}, v):=\mu\left(\left\{M \in X_{1}^{(d-1)}: \mathbb{Z}^{d-1} M \cap v^{-\frac{1}{d-1}} \mathfrak{C}_{\boldsymbol{h}}(\boldsymbol{z}, \boldsymbol{w})=\emptyset\right\}\right) .
$$

Our goal in the present section is to approximate the function $\Upsilon(\boldsymbol{z}, \boldsymbol{w}, \boldsymbol{h}, v)$ from above and below with the function $\Xi(a, b ; \boldsymbol{h} ; v)$.

When proving Theorem 1.7 we may without loss of generality assume $w \geq z$, since $\Phi_{\mathbf{0}}(\xi, w, z, \varphi)=$ $\Phi_{0}(\xi, z, w, \varphi)$ and also the right hand side of (1.29) is symmetric in $w, z$ (as will be seen from the definition (5.66), using (4.9) ). For given $0<z \leq w<1$ and $\varphi \in\left[0, \frac{\pi}{2}\right)$, we now make an explicit choice of corresponding vectors $\boldsymbol{w}=\left(w_{1}, \ldots, w_{d-1}\right), \boldsymbol{z}=\left(z_{1}, \ldots, z_{d-1}\right) \in \mathcal{B}_{1}^{d-1}$.

$$
\begin{aligned}
& \text { If } z>w \cos \varphi:\left\{\begin{array}{l}
\boldsymbol{z}=\frac{1}{\sqrt{z^{2}+w^{2}-2 z w \cos \varphi}}\left(z w(\sin \varphi) \boldsymbol{e}_{1}+z(z-w \cos \varphi) \boldsymbol{e}_{2}\right) \\
\boldsymbol{w}=\frac{1}{\sqrt{z^{2}+w^{2}-2 z w \cos \varphi}}\left(z w(\sin \varphi) \boldsymbol{e}_{1}-w(w-z \cos \varphi) \boldsymbol{e}_{2}\right) ;
\end{array}\right. \\
& \text { if } z \leq w \cos \varphi:\left\{\begin{array}{l}
\boldsymbol{z}=z \boldsymbol{e}_{1} \\
\boldsymbol{w}=w(\cos \varphi) \boldsymbol{e}_{1}-w(\sin \varphi) \boldsymbol{e}_{2} .
\end{array}\right.
\end{aligned}
$$

These $\boldsymbol{z}, \boldsymbol{w}$ are easily verified to satisfy $\|\boldsymbol{w}\|=w,\|\boldsymbol{z}\|=z$ and $\varphi(\boldsymbol{z}, \boldsymbol{w})=\varphi$. Note that $z>w \cos \varphi$ holds if and only if the triangle $\triangle \mathbf{0} \boldsymbol{z} \boldsymbol{w}$ is acute; the point of our choice of $\boldsymbol{z}, \boldsymbol{w}$ in this case is to make $z_{1}=w_{1}$ hold.

For $\boldsymbol{z}, \boldsymbol{w}$ given as above we now wish to approximate $\mathfrak{C}_{\boldsymbol{h}}(\boldsymbol{z}, \boldsymbol{w})$ by two cut paraboloids. In fact we will use two translates of the same paraboloid $P_{u, r}$ (cf. Sec. 3.5) , with $u=\sqrt{1-w_{1}^{2}}$ and appropriate $r$. This means that near the origin, $\boldsymbol{w}+P_{u, r}$ looks very much like $\boldsymbol{w}+\mathcal{B}_{1}^{d-1}$ (cf. Lemma 3.11 and note $w_{2} \leq 0$ ). Also $\boldsymbol{z}+P_{u, r}$ is in many cases a good approximation of $\boldsymbol{z}+\mathcal{B}_{1}^{d-1}$ near the origin; however if $z_{2}$ is near $\sqrt{1-z_{1}^{2}}$ then $\boldsymbol{z}+P_{u, r}$ may even fail to contain the origin, and a much better approximation of $\boldsymbol{z}+\mathcal{B}_{1}^{d-1}$ is given by $\boldsymbol{z}+\rho\left(P_{u, r}\right)$, where $\rho: \mathbb{R}^{d-1} \rightarrow \mathbb{R}^{d-1}$ denotes reflection in the hyperplane $\boldsymbol{e}_{2}^{\perp}$ (viz. $\rho\left(\left(x_{1}, \ldots, x_{d-1}\right)\right)=\left(x_{1},-x_{2}, x_{3}, \ldots, x_{d-1}\right)$ ). With $A=A(u, r), B=B(u, r)$ as in Section 3.5 we have

$$
\boldsymbol{z}+\rho\left(P_{u, r}\right)=\left(\boldsymbol{z}+\frac{B}{A} \boldsymbol{e}_{2}\right)+P_{u, r} .
$$

In fact we will use $\boldsymbol{z}+\rho\left(P_{u, r}\right)$ in place of $\boldsymbol{z}+P_{u, r}$ if and only if $\varphi^{2} \geq 3(1-z)$. In view of the above relation, it is convenient to introduce the point

$$
\boldsymbol{z}^{\prime}=\boldsymbol{z}_{z, \varphi, u, r}^{\prime}=\left(z_{1}^{\prime}, z_{2}^{\prime}, \ldots, z_{d-1}^{\prime}\right):= \begin{cases}\boldsymbol{z}+\frac{B}{A} \boldsymbol{e}_{2} & \text { if } \varphi^{2} \geq 3(1-z) \\ \boldsymbol{z} & \text { otherwise }\end{cases}
$$


Note that $\varphi^{2} \geq 3(1-z)$ can only happen in the first of the two cases in (4.37), since $z \leq w \cos \varphi$ implies $z \leq w \cos \varphi<\cos \varphi<1-\frac{1}{3} \varphi^{2}$. Note also that if $r=-u$ then $B=0$ and $\boldsymbol{z}^{\prime}=\boldsymbol{z}$, and by Lemma 3.12(i) we have

$$
\mathfrak{C}_{\boldsymbol{h}}(\boldsymbol{z}, \boldsymbol{w}) \subset\left(\left(\boldsymbol{z}^{\prime}+P_{u,-u}\right) \cup\left(\boldsymbol{w}+P_{u,-u}\right)\right) \cap \mathbb{R}_{\boldsymbol{h}-}^{d-1} .
$$

The following lemma gives conditions for the opposite inclusion to hold:

Lemma 4.10. There is an absolute constant $c_{9}>0$ such that whenever $\frac{9}{10} \leq z \leq w<1$, $0 \leq \varphi \leq \frac{1}{10}, \boldsymbol{h}=\left(h_{1}, \ldots, h_{d-1}\right) \in \mathbb{R}_{+}^{d-1}, 0 \leq u<r \leq \frac{1}{2}$, and

$$
r \geq c_{9}\left(\sqrt{1-z}+\varphi+\frac{\left\|\boldsymbol{h}^{\prime}\right\|}{\|\boldsymbol{h}\|}\right) \quad\left(\text { with } \boldsymbol{h}^{\prime}:=\left(h_{2}, \ldots, h_{d-1}\right)\right),
$$

then we have, for $\boldsymbol{w}, \boldsymbol{z}$ as in (4.37) and $\boldsymbol{z}^{\prime}$ as in (4.39),

$$
\left(\left(\boldsymbol{z}^{\prime}+P_{u, r}\right) \cup\left(\boldsymbol{w}+P_{u, r}\right)\right) \cap \mathbb{R}_{\boldsymbol{h}-}^{d-1} \subset \mathfrak{C}_{\boldsymbol{h}}(\boldsymbol{z}, \boldsymbol{w}) .
$$

Proof. Recalling the definition of $\mathfrak{C}_{\boldsymbol{h}}(\boldsymbol{z}, \boldsymbol{w})$ we see that it suffices to prove

$$
\left(\boldsymbol{z}^{\prime}+P_{u, r}\right) \cap \mathbb{R}_{\boldsymbol{h}-}^{d-1} \subset \boldsymbol{z}+\mathcal{B}_{1}^{d-1} \quad \text { and } \quad\left(\boldsymbol{w}+P_{u, r}\right) \cap \mathbb{R}_{\boldsymbol{h}-}^{d-1} \subset \boldsymbol{w}+\mathcal{B}_{1}^{d-1} .
$$

In view of (4.38) and Lemma 3.12(ii), in order to prove the first inclusion in (4.42) it suffices to prove

$$
\left(\boldsymbol{z}^{\prime}+P_{u, r}\right) \cap \mathbb{R}_{\boldsymbol{h}-}^{d-1} \subset\left\{x_{1}<z_{1}-\sqrt{1-r^{2}}\right\} .
$$

Without loss of generality we may rescale $\boldsymbol{h}$ so that $h_{1}=1$. But we have by (3.59),

$$
P_{u, r}=\left(C-\frac{B^{2}}{4 A}\right) \boldsymbol{e}_{1}-\frac{B}{2 A} \boldsymbol{e}_{2}+\left\{x_{1}>A\left(x_{2}^{2}+\ldots+x_{d-1}^{2}\right)\right\} .
$$

Also the image of the halfspace $\mathbb{R}_{\boldsymbol{h}-}^{d-1}$ under the translation $\boldsymbol{x} \mapsto \boldsymbol{x}-\boldsymbol{z}^{\prime}-\left(C-\frac{B^{2}}{4 A}\right) \boldsymbol{e}_{1}+\frac{B}{2 A} \boldsymbol{e}_{2}$ is computed to equal $\lambda e_{1}+\mathbb{R}_{h-}^{d-1}$, where

$$
\lambda:=-z_{1}-C+\frac{B^{2}}{4 A}+h_{2}\left(\frac{B}{2 A}-z_{2}^{\prime}\right) .
$$

Hence (4.43) is equivalent with

$$
\left\{x_{1}>A\left(x_{2}^{2}+\ldots+x_{d-1}^{2}\right)\right\} \cap\left(\lambda \boldsymbol{e}_{1}+\mathbb{R}_{\boldsymbol{h}-}^{d-1}\right) \subset\left\{x_{1}<-C+\frac{B^{2}}{4 A}-\sqrt{1-r^{2}}\right\} .
$$

Note that $0<A \asymp 1$ (cf. (3.59) and recall $0 \leq u<r \leq \frac{1}{2}$ ). Using the we compute that the supremum of $x_{1}$ taken over all points $\boldsymbol{x}$ lying in the set in the left hand side of (4.44) is

$$
=(4 A)^{-1}\left(\left\|\boldsymbol{h}^{\prime}\right\|+\left(\left\|\boldsymbol{h}^{\prime}\right\|^{2}+4 A \lambda\right)^{1 / 2}\right)^{2} \leq \lambda+O\left(\left\|\boldsymbol{h}^{\prime}\right\|^{2}+\left\|\boldsymbol{h}^{\prime}\right\| \sqrt{\max (0, \lambda)}\right) .
$$

(This presupposes $\left\|\boldsymbol{h}^{\prime}\right\|^{2}+4 A \lambda>0$; in the opposite case the set in the left hand side of (4.44) is empty, so that the desired inclusion holds trivially.)

Now note that if $z>w \cos \varphi$ then $0 \leq w-z<w(1-\cos \varphi) \leq \frac{1}{2} \varphi^{2}$, and hence

$$
z^{2}+w^{2}-2 z w \cos \varphi=(w-z)^{2}+2 z w(1-\cos \varphi)=\varphi^{2}\left(1+O\left(1-z+\varphi^{2}\right)\right) .
$$

The same computation also shows

$$
z^{2}+w^{2}-2 z w \cos \varphi \gg \varphi^{2}
$$

Using (4.46), (4.47) and (4.37) we obtain

$$
1-z_{1} \asymp 1-z_{1}^{2} \ll 1-z+\varphi^{2} .
$$

This bound is obviously also true when $z \leq w \cos \varphi$, cf. (4.37), i.e. it is true in general. We also get from (4.37) (using $z-w \cos \varphi \leq w(1-\cos \varphi)$ ),

$$
0 \leq z_{2} \ll \varphi .
$$

Also note that $D \ll r^{2}, A \asymp 1,|B| \ll r^{3}$ and $C=-1+O\left(r^{2}\right)$ (cf. (3.59) and recall $0 \leq u<$ $\left.r \leq \frac{1}{2}\right)$, and $\frac{B}{2 A}-z_{2}^{\prime}=\left( \pm \frac{B}{2 A}\right)-z_{2}$. 
It follows from the above observations that (4.45) is

$$
\leq-C+\frac{B^{2}}{4 A}-1+O\left(\left(\sqrt{1-z}+\varphi+\left\|\boldsymbol{h}^{\prime}\right\|\right)\left(\sqrt{1-z}+\varphi+\left\|\boldsymbol{h}^{\prime}\right\|+r\right)\right) .
$$

This has been proved under the assumptions $\frac{9}{10} \leq z \leq w<1,0 \leq \varphi \leq \frac{1}{10}, \boldsymbol{h} \in \mathbb{R}_{+}^{d-1}, h_{1}=1$ and $0 \leq u<r \leq \frac{1}{2}$. Now if we also assume $c_{9}\left(\sqrt{1-z}+\varphi+\frac{\left\|\boldsymbol{h}^{\prime}\right\|}{\|\boldsymbol{h}\|}\right) \leq r \leq \frac{1}{2}$ where $c_{9}$ is a sufficiently large constant (this in particular forces $\frac{\left\|\boldsymbol{h}^{\prime}\right\|}{\|\boldsymbol{h}\|}$ to be small; hence $\|\boldsymbol{h}\| \asymp h_{1}=1$ ), then it follows that the big- $O$-term in (4.45) is $<\frac{1}{2} r^{2}$, and since $\frac{1}{2} r^{2}<\frac{r^{2}}{1+\sqrt{1-r^{2}}}=1-\sqrt{1-r^{2}}$ this implies that (4.44) holds.

This completes the proof of the first inclusion in (4.42); the second inclusion is proved by a completely similar argument.

Next, in order to relate (4.40) and (4.41) to the function $\Xi(a, b ; \boldsymbol{h} ; v)$, we need to transform $\left(\left(\boldsymbol{z}^{\prime}+P_{u, r}\right) \cup\left(\boldsymbol{w}+P_{u, r}\right)\right) \cap \mathbb{R}_{h-}^{d-1}$ by a linear map into a union of the form $P_{\boldsymbol{h}}^{d-1}(\mathbf{0}) \cup P_{\boldsymbol{h}}^{d-1}\left(\mathbf{0} T_{a, b}\right)$, cf. (4.2) and (4.8). The following lemma gives a detailed description of the parameters occurring in this transformation.

Lemma 4.11. There is an absolute constant $c_{10} \in\left(0, \frac{1}{10}\right]$ such that for any fixed $z, w, \varphi$ with $1-c_{10} \leq z \leq w<1,0 \leq \varphi \leq c_{10}$, there exist a function $M:\left[-c_{10}, c_{10}\right] \rightarrow \operatorname{GL}(d-1, \mathbb{R})$ and $\mathrm{C}^{1}$ functions $a, \alpha:\left[-c_{10}, c_{10}\right] \rightarrow \mathbb{R}_{>0}, b:\left[-c_{10}, c_{10}\right] \rightarrow \mathbb{R}_{\geq 0}, \beta:\left[-c_{10}, c_{10}\right] \rightarrow \mathbb{R}$, such that for $\boldsymbol{z}, \boldsymbol{w}$ as in (4.37), $\boldsymbol{z}^{\prime}$ as in (4.39), $u=\sqrt{1-w_{1}^{2}}$ and arbitrary $r \in\left[-c_{10}, c_{10}\right]$ and $\boldsymbol{h} \in \mathbb{R}_{+}^{d-1}$, we have, writing $M=M(r), a=a(r), b=b(r), \alpha=\alpha(r), \beta=\beta(r)$ :

$$
\left(\left(\left(\boldsymbol{z}^{\prime}+P_{u, r}\right) \cup\left(\boldsymbol{w}+P_{u, r}\right)\right) \cap \mathbb{R}_{\boldsymbol{h}-}^{d-1}\right) M=P_{\boldsymbol{k}}^{d-1}(\mathbf{0}) \cup P_{\boldsymbol{k}}^{d-1}\left(\mathbf{0} T_{a, b}\right)
$$

where

$$
\boldsymbol{k}=\left(\alpha h_{1}, 2 \beta h_{1}+h_{2}, h_{3}, \ldots, h_{d-1}\right),
$$

and furthermore, with $R=(1-z)+\varphi^{2}+r^{2}$ and with absolute implied constants:

$$
\begin{array}{lll}
a=\sqrt{\frac{1-w}{1-z}}(1+O(R)) ; & \left|\frac{\partial a}{\partial r}\right| \ll \sqrt{\frac{1-w}{1-z}} \sqrt{R} ; \\
b=\frac{\varphi}{\sqrt{2(1-z)}}(1+O(R)) ; & \left|\frac{\partial b}{\partial r}\right| \ll \frac{\varphi}{\sqrt{1-z}} \sqrt{R} ; \\
\alpha=\sqrt{\frac{1}{2}(1-z)}(1+O(R)) ; & \left|\frac{\partial \alpha}{\partial r}\right| \ll \sqrt{1-z} \sqrt{R} ; \\
|\beta| \ll \sqrt{1-z+\varphi^{2}} ; & \left|\frac{\partial \beta}{\partial r}\right| \ll R ; \\
0<\operatorname{det} M=2^{1-\frac{d}{2}}(1-z)^{-\frac{d}{2}}(1+O(R)) . &
\end{array}
$$

As an auxiliary lemma, let us first note the following regarding the functions $A=A(u, r)$, $B=B(u, r), C=C(u, r), D=D(u, r)$ introduced in Section 3.5.

Lemma 4.12. The following bounds holds uniformly over all $u, r$ with $|u|,|r| \leq \frac{1}{2}$ :

$\left|\frac{\partial D}{\partial r}\right| \ll|u|+|r|, \quad\left|\frac{\partial A}{\partial r}\right| \ll|u|+|r|, \quad\left|\frac{\partial B}{\partial r}\right| \ll|u|(|u|+|r|), \quad\left|\frac{\partial C}{\partial r}\right| \ll u^{2}(|u|+|r|)$.

Proof. The bound $\left|\frac{\partial D}{\partial r}\right| \ll|u|+|r|$ is proved by a direct computation and the other three bounds follow trivially from this.

Proof of Lemma 4.11. Let $z, w, \varphi$ (and thus $\boldsymbol{w}, \boldsymbol{z}, \boldsymbol{z}^{\prime}$ ) be given as in the formulation of the lemma, and set $u=\sqrt{1-w_{1}^{2}}$. Also set $\epsilon:=-1$ if $\varphi^{2} \geq 3(1-z)$ and otherwise $\epsilon:=1$, so 
that $\boldsymbol{z}^{\prime}=\boldsymbol{z}+\frac{1}{2}(1-\epsilon) \frac{B}{A} \boldsymbol{e}_{2}$. Now for any $r \in\left[-c_{10}, c_{10}\right]$ we let $A=A(u, r), B=B(u, r)$, $C=C(u, r), D=D(u, r)$ be as in Section 3.5, define $T_{1} \in \operatorname{AGL}(d-1, \mathbb{R})$ by

$$
\boldsymbol{x} T_{1}=\left(x_{1}, \ldots, x_{d-1}\right) T_{1}:=\left(A x_{1}-1-A C+\frac{1}{4} B^{2}, A x_{2}+\frac{1}{2} B, A x_{3}, \ldots, A x_{d-1}\right),
$$

and set

$$
\begin{aligned}
& \boldsymbol{y}:=\left(-\boldsymbol{z}^{\prime}\right) T_{1}=\left(-A z_{1}-1-A C+\frac{1}{4} B^{2}\right) \boldsymbol{e}_{1}+\left(\epsilon \frac{1}{2} B-A z_{2}\right) \boldsymbol{e}_{2} \\
& \boldsymbol{y}^{\prime}:=(-\boldsymbol{w}) T_{1}=\left(-A w_{1}-1-A C+\frac{1}{4} B^{2}\right) \boldsymbol{e}_{1}+\left(\frac{1}{2} B-A w_{2}\right) \boldsymbol{e}_{2} .
\end{aligned}
$$

Finally define $\alpha=\alpha(r), \beta=\beta(r), a=a(r), b=b(r)$ as in Lemma 4.1, applied with $\boldsymbol{y}, \boldsymbol{y}^{\prime}$ as in (4.55) (we will see that $\boldsymbol{y}, \boldsymbol{y}^{\prime}$ lie in $P^{d-1}$ provided that $c_{10}$ is sufficiently small; cf. (4.63) and (4.66) below), and set $T=T_{1} T_{\alpha, \beta}^{-1}$ and

$$
M=M(r):=\operatorname{diag}[A, \ldots, A] M_{\alpha, \beta}^{-1} .
$$

Then by construction we have $T=(M, \boldsymbol{\xi})$ for some $\boldsymbol{\xi} \in \mathbb{R}^{d-1} ;\left(-\boldsymbol{z}^{\prime}\right) T=\mathbf{0} ;(-\boldsymbol{w}) T=\mathbf{0} T_{a, b}$, and $P_{u, r} T=P^{d-1}$. Also by a quick computation one checks that $\mathbb{R}_{\boldsymbol{h}-}^{d-1} M=\mathbb{R}_{\boldsymbol{k}-}^{d-1}$ for all $\boldsymbol{h} \in \mathbb{R}_{+}^{d-1}$, with $\boldsymbol{k}$ as in (4.52). Hence (4.51) holds.

It remains to verify that the functions $M, a, b, \alpha, \beta$ have the properties stated in (4.53). Writing $\boldsymbol{y}=y_{1} \boldsymbol{e}_{1}+y_{2} \boldsymbol{e}_{2}$ and $\boldsymbol{y}^{\prime}=y_{1}^{\prime} \boldsymbol{e}_{1}+y_{2}^{\prime} \boldsymbol{e}_{2}$, we compute

$$
\begin{aligned}
& 1+y_{1}-y_{2}^{2}=A\left(-z_{1}-A z_{2}^{2}+\epsilon B z_{2}-C\right)=\frac{A}{2 w_{1}}\left(1+w_{1}^{2}-2 w_{1} z_{1}-z_{2}^{2}-D\left(u+\epsilon z_{2}\right)^{2}\right) ; \\
& 1+y_{1}^{\prime}-y_{2}^{\prime 2}=A\left(-w_{1}-A w_{2}^{2}+B w_{2}-C\right)=\frac{A}{2 w_{1}}\left(1-w^{2}-D\left(u+w_{2}\right)^{2}\right) .
\end{aligned}
$$

Note that $w_{1} \geq z_{1}$ always holds, and recall (4.48) in the proof of Lemma 4.10. It follows that

$$
u^{2}=1-w_{1}^{2} \ll 1-z+\varphi^{2} .
$$

Hence assuming that $c_{10}$ has been taken sufficiently small we have $0 \leq u \leq \frac{1}{2}$, and since also $|r| \leq c_{10} \leq \frac{1}{10}$, (3.60) implies

$$
D \ll u^{2}+r^{2} .
$$

We also get (cf. (3.59))

$$
A=\frac{1}{2}+O(R) \quad \text { and } \quad \frac{A}{w_{1}}=\frac{1}{2}+O(R) .
$$

Now if $z>w \cos \varphi$ then we obtain from (4.57):

$$
1+y_{1}-y_{2}^{2}=\frac{A}{2 w_{1}}\left(1-z^{2}-D\left(u+\epsilon z_{2}\right)^{2}\right),
$$

and here if $\epsilon=-1$ then (since $0<z_{2}<u$ )

$$
u+\epsilon z_{2}=\frac{1-z^{2}}{\sqrt{1-z^{2}+z_{2}^{2}}+z_{2}} \leq \sqrt{1-z^{2}},
$$

while if $\epsilon=1$ (thus $\varphi^{2} \ll 1-z$ ) then we still have $u+\epsilon z_{2}<2 u \ll \sqrt{1-z}$. Hence always when $z>w \cos \varphi$ we get, using also (4.59) and (4.60),

$$
1+y_{1}-y_{2}^{2}=\frac{1}{2}(1-z)(1+O(R)) .
$$

On the other hand if $z \leq w \cos \varphi$ then we have $\varphi^{2}<3(1-z)$ as noted below (4.39), and also $z_{2}=0$ and

$$
1+y_{1}-y_{2}^{2}=\frac{A}{2 w_{1}}\left(\left(1-w_{1}\right)^{2}+2 w_{1}(1-z)-D u^{2}\right) .
$$


Hence using (4.58), (4.59) and (4.60) we see that (4.63) again holds; thus (4.63) is true in general. By a similar discussion, also using Lemma 4.12, we find that, both when $z>w \cos \varphi$ and when $z \leq w \cos \varphi$ :

$$
\left|\frac{\partial}{\partial r}\left(1+y_{1}-y_{2}^{2}\right)\right| \ll(1-z) \sqrt{R} .
$$

By similar computations (using $u+w_{2}=\frac{1-w^{2}}{\sqrt{1-w_{1}^{2}}+\left|w_{2}\right|} \leq \sqrt{1-w^{2}}$ ) we also have

$$
1+y_{1}^{\prime}-{y_{2}^{\prime}}^{2}=\frac{1}{2}(1-w)(1+O(R)) ; \quad\left|\frac{\partial}{\partial r}\left(1+y_{1}^{\prime}-y_{2}^{\prime 2}\right)\right| \ll(1-w) \sqrt{R} .
$$

We next study the difference $y_{2}^{\prime}-y_{2}$. By (4.55),

$$
y_{2}^{\prime}-y_{2}=\frac{1}{2}(1-\epsilon) B+A\left(z_{2}-w_{2}\right) .
$$

If $z>w \cos \varphi$, then assuming $c_{10}$ sufficiently small and using (4.46) and (4.60) we have

$$
A\left(z_{2}-w_{2}\right)=A \sqrt{z^{2}+w^{2}-2 z w \cos \varphi}=\frac{1}{2} \varphi(1+O(R)) .
$$

Note also that if $\epsilon=-1$ then $1-z \ll \varphi^{2}$ and thus by (3.59), (4.58) and (4.59), $|B| \ll D u \ll$ $R \varphi$. Hence always when $z>w \cos \varphi$ we have

$$
y_{2}^{\prime}-y_{2}=\frac{1}{2} \varphi(1+O(R)) .
$$

On the other hand if $z \leq w \cos \varphi$ then $\epsilon=1$ and $z_{2}-w_{2}=w \sin \varphi=\varphi(1+O(R))$; hence (4.69) again holds, i.e. (4.69) is true in general. By similar considerations, also using Lemma 4.12, we find that, both when $z>w \cos \varphi$ and when $z \leq w \cos \varphi$,

$$
\left|\frac{\partial}{\partial r}\left(y_{2}^{\prime}-y_{2}\right)\right| \ll \varphi \sqrt{R}
$$

Note that by assuming $c_{10}$ to be sufficiently small we can force the big- $O$ terms in (4.63) and (4.66) to be less than $\frac{1}{2}$ in absolute value. Hence the first three lines of (4.53) now follow from Lemma 4.1 combined with (4.63), (4.65), (4.66), (4.69), (4.70). The fourth line of (4.53) follows from $\beta=y_{2}=\epsilon \frac{1}{2} B-A z_{2},|B| \ll u, A \ll 1,0 \leq z_{2}<u \ll \sqrt{1-z+\varphi^{2}}$, and Lemma 4.12, Finally the last line of (4.53) follows by also using $\operatorname{det} M=A^{d-1} \alpha^{-d}=A^{d-1}\left(1+y_{1}-y_{2}^{2}\right)^{-\frac{d}{2}}$, cf. (4.7) and Lemma 4.1.

In the next two propositions we give the desired approximations of $\Upsilon(\boldsymbol{z}, \boldsymbol{w}, \boldsymbol{h}, v)$ in terms of the $\Xi$-function. We start with the approximation from above, which is in some respects more complicated than the one from below.

Proposition 4.13. There exist constants $c_{11} \in\left(0, \frac{1}{10}\right]$ and $c_{12}>1$ which only depend on $d$ such that for any fixed $z, w, \varphi$ with $1-c_{11} \leq z \leq w<1,0 \leq \varphi \leq c_{11}$, there exist $\mathrm{C}^{1}$ functions $\alpha:\left[0, c_{11}\right] \rightarrow \mathbb{R}_{>0}$ and $\beta:\left[0, c_{11}\right] \rightarrow \mathbb{R}$ which satisfy the bounds

$$
\begin{array}{ll}
\frac{1}{2} \sqrt{1-z}<\alpha(s)=\sqrt{\frac{1}{2}(1-z)}\left(1+O\left(1-z+\varphi^{2}+s^{2}\right)\right) ; & \left|\alpha^{\prime}(s)\right| \ll \sqrt{1-z} \sqrt{1-z+\varphi^{2}+s^{2}} ; \\
|\beta(s)| \ll \sqrt{1-z+\varphi^{2}} ; & \left|\beta^{\prime}(s)\right| \ll 1-z+\varphi^{2}+s^{2},
\end{array}
$$

for all $s \in\left[0, c_{11}\right]$, and which have the property that, for $\boldsymbol{z}, \boldsymbol{w}$ as in (4.37), and for all $v>0$ and all $\boldsymbol{h} \in \mathbb{R}_{+}^{d-1}$ with $\frac{\left\|\boldsymbol{h}^{\prime}\right\|}{\|\boldsymbol{h}\|} \leq c_{11}$,

$$
\begin{array}{r}
\Upsilon(\boldsymbol{z}, \boldsymbol{w}, \boldsymbol{h}, v) \leq \Xi\left(\sqrt{\frac{1-w}{1-z}}, \frac{\varphi}{\sqrt{2(1-z)}} ;\left(\alpha\left(\frac{\left\|\boldsymbol{h}^{\prime}\right\|}{\|\boldsymbol{h}\|}\right) h_{1}, 2 \beta\left(\frac{\left\|\boldsymbol{h}^{\prime}\right\|}{\|\boldsymbol{h}\|}\right) h_{1}+h_{2}, h_{3}, \ldots, h_{d-1}\right) ;\right. \\
\left.2^{1-\frac{d}{2}}(1-z)^{-\frac{d}{2}} v\left\{1+c_{12}\left(1-z+\varphi^{2}+\frac{\left\|\boldsymbol{h}^{\prime}\right\|^{2}}{\|\boldsymbol{h}\|^{2}}\right)\right\}\right) .
\end{array}
$$


Proof. We will use the constants $c_{10}$ from Lemma 4.11 and $c_{9}$ from Lemma4.10. After possibly enlargening $c_{9}$ we may assume that $\sqrt{1-w_{1}^{2}}<c_{9}(\sqrt{1-z}+\varphi)$ whenever $1-c_{10} \leq z \leq w<1$, $0 \leq \varphi \leq c_{10}(\mathrm{cf}$. (4.58) $)$. Take $c_{11} \in\left(0, c_{10}\right]$ so small that $c_{9}\left(\sqrt{c_{11}}+2 c_{11}\right) \leq c_{10}$.

Now let $z, w, \varphi$ (and thus $\boldsymbol{w}, \boldsymbol{z})$ be given subject to $1-c_{11} \leq z \leq w<1$ and $0 \leq \varphi \leq c_{11}$. Let us write $a, \alpha_{0}:\left[-c_{10}, c_{10}\right] \rightarrow \mathbb{R}_{>0}, b:\left[-c_{10}, c_{10}\right] \rightarrow \mathbb{R}_{\geq 0}, \beta_{0}:\left[-c_{10}, c_{10}\right] \rightarrow \mathbb{R}$ for the functions provided by Lemma 4.11 , Now for $s \in\left[0, c_{11}\right]$ we set

$$
r=r(s):=c_{9}(\sqrt{1-z}+\varphi+s) ; \quad \alpha_{1}(s)=\alpha_{0}(r(s)) ; \quad \beta_{1}(s)=\beta_{0}(r(s)) .
$$

(Note that $r \leq c_{10} \leq \frac{1}{10}$ for all $s \in\left[0, c_{11}\right]$ because of our choice of $c_{11}$; in particular $\alpha_{1}(s)$, $\beta_{1}(s)$ are well-defined for all $s \in\left[0, c_{11}\right]$.) We set $u=\sqrt{1-w_{1}^{2}}$ as in Lemma 4.11; note that $u<r(s)$ for all $s \in\left[0, c_{11}\right]$, because of our choice of $c_{9}$.

Now let $\boldsymbol{h} \in \mathbb{R}_{+}^{d-1}$ be given, subject to $\frac{\left\|\boldsymbol{h}^{\prime}\right\|}{\|\boldsymbol{h}\|} \leq c_{11}$. Set $s=\frac{\left\|\boldsymbol{h}^{\prime}\right\|}{\|\boldsymbol{h}\|}, r=r(s)$ and $M=M(r(s))$, where $M:\left[-c_{10}, c_{10}\right] \rightarrow \mathrm{GL}(d-1, \mathbb{R})$ is as in Lemma 4.11, then

$$
\left(\left(\left(\boldsymbol{z}^{\prime}+P_{u, r}\right) \cup\left(\boldsymbol{w}+P_{u, r}\right)\right) \cap \mathbb{R}_{\boldsymbol{h}-}^{d-1}\right) M=P_{\boldsymbol{k}}^{d-1}(\mathbf{0}) \cup P_{\boldsymbol{k}}^{d-1}\left(\mathbf{0} T_{a, b}\right)
$$

with $a=a(r), b=b(r)$ and

$$
\boldsymbol{k}:=\left(\alpha_{1}(s) h_{1}, 2 \beta_{1}(s) h_{1}+h_{2}, h_{3}, \ldots, h_{d-1}\right) .
$$

Also by Lemma 4.10 we have

$$
\left(\left(\boldsymbol{z}^{\prime}+P_{u, r}\right) \cup\left(\boldsymbol{w}+P_{u, r}\right)\right) \cap \mathbb{R}_{\boldsymbol{h}-}^{d-1} \subset \mathfrak{C}_{\boldsymbol{h}}(\boldsymbol{z}, \boldsymbol{w}) .
$$

Hence, recalling (4.2), (4.8), (4.36) and the fact that $\mu$ is $G$-invariant,

$$
\Upsilon(\boldsymbol{z}, \boldsymbol{w}, \boldsymbol{h}, v) \leq \Xi(a, b ; \boldsymbol{k} ;(\operatorname{det} M) v) .
$$

Using now also Lemma 4.9 it follows, assuming that $\tau_{a}:=a^{-1} \sqrt{\frac{1-w}{1-z}}$ and $\tau_{b}:=b^{-1} \frac{\varphi}{\sqrt{2(1-z)}}$ (if $\left.\varphi=0: \tau_{b}:=1\right)$ both lie in $\left[\frac{1}{2}, \frac{3}{2}\right]$, that

$$
\Xi(a, b ; \boldsymbol{k} ;(\operatorname{det} M) v) \leq \Xi\left(\sqrt{\frac{1-w}{1-z}}, \frac{\varphi}{\sqrt{2(1-z)}} ;\left(\tau_{b}^{-1} k_{1}, k_{2}, \ldots, k_{d-1}\right) ; v^{\prime}\right),
$$

where $v^{\prime}=(\operatorname{det} M) v\left(1+O\left(\left|\tau_{a}-1\right|+\left|\tau_{b}-1\right|\right)\right)$.

But from Lemma 4.11 we know that $\left|\tau_{a}-1\right|,\left|\tau_{b}-1\right| \ll 1-z+\varphi^{2}+r^{2}$; hence $\mid \tau_{a}-$ $1|,| \tau_{b}-1 \mid \ll 1-z+\varphi^{2}+s^{2}$, and thus after possibly shrinking $c_{11}$ we can ensure that $\left|\tau_{a}-1\right|,\left|\tau_{b}-1\right| \leq \frac{1}{2}$ always hold for our $\boldsymbol{w}, \boldsymbol{z}, \boldsymbol{h}$. Hence we see, using also the fact that $\operatorname{det} M=2^{1-\frac{d}{2}}(1-z)^{-\frac{d}{2}}\left(1+O\left(1-z+\varphi^{2}+s^{2}\right)\right)$ (cf. Lemma 4.11), that (4.72) holds, if we define

$$
\alpha(s)=\frac{\sqrt{2(1-z)} b(r(s))}{\varphi} \alpha_{1}(s) ; \quad \text { and } \quad \beta(s)=\beta_{1}(s), \quad \forall s \in\left[0, c_{11}\right] .
$$

(If $\varphi=0$ then $b \equiv 0$, and we set $\alpha(s)=\alpha_{1}(s)$.) Finally the properties in (4.71) follow directly from our definitions and Lemma 4.11 (taking $c_{11}$ sufficiently small).

Proposition 4.14. There exist constants $c_{13} \in\left(0, \frac{1}{10}\right]$ and $c_{14}>1$ which only depend on $d$ such that for any fixed $z, w, \varphi$ with $1-c_{13} \leq z \leq w<1,0 \leq \varphi \leq c_{13}$, there exist real numbers $\alpha, \beta$ which satisfy the bounds

$$
\frac{1}{2} \sqrt{1-z}<\alpha=\sqrt{\frac{1}{2}(1-z)}\left(1+O\left(1-z+\varphi^{2}\right)\right) ; \quad|\beta| \ll \sqrt{1-z+\varphi^{2}},
$$


and which have the property that, for $\boldsymbol{z}, \boldsymbol{w}$ as in (4.37), and for all $v>0$ and all $\boldsymbol{h} \in \mathbb{R}_{+}^{d-1}$,

$$
\begin{array}{r}
\Upsilon(\boldsymbol{z}, \boldsymbol{w}, \boldsymbol{h}, v) \geq \Xi\left(\sqrt{\frac{1-w}{1-z}}, \frac{\varphi}{\sqrt{2(1-z)}} ;\left(\alpha h_{1}, 2 \beta h_{1}+h_{2}, h_{3}, \ldots, h_{d-1}\right) ;\right. \\
\left.2^{1-\frac{d}{2}}(1-z)^{-\frac{d}{2}} v\left(1-c_{14}\left(1-z+\varphi^{2}\right)\right)^{+}\right),
\end{array}
$$

where $x^{+}:=\max (0, x)$.

Proof. This is very similar to the proof of Proposition 4.13, except that we apply Lemma 4.11 with $r=-u=-\sqrt{1-w_{1}^{2}}$ (independent of $\boldsymbol{h}$ ); in place of Lemma 4.10 we simply use (4.40) to obtain $\Upsilon(\boldsymbol{z}, \boldsymbol{w}, \boldsymbol{h}, v) \geq \Xi(a, b ; \boldsymbol{k} ;(\operatorname{det} M) v)$; and finally we apply Lemma 4.9 with $\tau_{a}:=a \sqrt{\frac{1-z}{1-w}}$ and $\tau_{b}:=b \frac{\sqrt{2(1-z)}}{\varphi}$, and $\sqrt{\frac{1-z}{1-w}}, \frac{\sqrt{2(1-z)}}{\varphi}$ in place of $a, b$.

\section{Asymptotics For $\Phi_{\mathbf{0}}(\xi, \boldsymbol{w}, \boldsymbol{z})$ AS $\xi \rightarrow \infty$}

We now start with the proof of Theorem 1.7. The proof involves approximating $\Phi_{\mathbf{0}}(\xi, \boldsymbol{w}, \boldsymbol{z})$ with an integral involving the $\Upsilon$-function (cf. (5.27) below, as well as (4.36)), which is then estimated from above and below in terms of the $\Xi$-function, using Propositions 4.13 and 4.14 , The resulting integral is then made cleaner in a series of steps, eventually resulting in the function $F_{\mathbf{0}, d}$ which we define in (5.66) below.

5.1. Initial reductions. Note that if $d=2$ then Theorem 1.7 (with $F_{\mathbf{0}, 2}$ as in (1.31)) follows directly from the explicit formula in [22]. Hence we will from now on assume $d \geq 3$.

As pointed out in Section 4.4 we may assume $w \geq z$ without loss of generality. Let us fix the constant $c_{3}$ so that $c_{3} \geq \sqrt{\sigma_{d}(1,0)}$ and $\Phi_{\mathbf{0}}(\xi, w, z, \varphi)=0$ whenever $1-z \geq c_{3} \xi^{-\frac{2}{d}}$ (cf. Proposition (1.10); these conditions are equivalent to the conditions imposed on $c_{3}$ at the start of Section 3.4. It will be clear from the definition of $F_{\mathbf{0}, d}$ in (5.66) that $F_{\mathbf{0}, d}\left(t_{1}, t_{2}, \alpha\right)=0$ holds whenever $t_{1} \geq \sqrt{\sigma_{d}(1,0)}$ (for recall (4.13), (4.14), and $\Xi(a, b ; \boldsymbol{h} ; v) \leq \Xi\left(\frac{\left\|\boldsymbol{h}^{\prime}\right\|}{h_{1}} ; v\right)$ ). Thus (1.29) is automatic when $1-z \geq c_{3} \xi^{-\frac{2}{d}}$. Hence from now on we will assume $1-z<c_{3} \xi^{-\frac{2}{d}}$.

Let $c_{15}$ be a positive constant which is smaller than both $c_{11}$ from Proposition 4.13 and $c_{13}$ from Proposition 4.14. (We will later impose some further conditions on $c_{15}$ being sufficiently small, but we will see that it can be fixed in a way which only depends on $d$.) If $c_{15}<\varphi \leq \frac{\pi}{2}$ then by Theorem 1.9 and (5.66) coupled with Lemma 5.3 below, both $\Phi_{0}(\xi, w, z, \varphi)$ and the main term in the right hand side of (1.29) are $\ll \xi^{-3+\frac{2}{d-1}}$, and thus (1.29) is automatically true. Hence from now on we will assume $0 \leq \varphi \leq c_{15}$.

By Lemma 3.1 there is a constant $0<c_{4}<\frac{1}{2}$ which only depends on $d$ such that for any $\xi>0$ and any translate $\mathfrak{Z}$ of the cylinder $\xi^{\frac{1}{d}} \mathfrak{Z}(0,1,1)$, we have that $a_{1}>A:=c_{4} \xi^{\frac{1}{d}}$ holds for all $M \in \mathcal{S}_{d}$ with $\mathbb{Z}^{d} M \cap \mathfrak{Z}=\emptyset$. We will assume from start that $\xi>\max \left(1,\left(c_{3} / c_{15}\right)^{d / 2}, c_{4}^{-d}\right)$; in particular we have $1-c_{15}<z<1$ and $A>1$. For later reference we recapitulate our main assumptions on $z, w, \varphi$ :

$$
1-z \geq c_{3} \xi^{-\frac{2}{d}} ; \quad 1-c_{15}<z \leq w<1 ; \quad 0 \leq \varphi \leq c_{15} .
$$

We fix $\boldsymbol{z}, \boldsymbol{w}$ as in (4.37), for our given $z, w, \varphi$.

Let $\mathcal{F}_{d} \subset \mathcal{S}_{d}^{\prime}$ be a fundamental region for $\Gamma \backslash G$ as in Lemma 3.4 (applied with our $A=c_{4} \xi^{\frac{1}{d}}$ ). By [29, (7.32)] we have

$$
\Phi_{\mathbf{0}}(\xi, \boldsymbol{w}, \boldsymbol{z})=\sum_{\boldsymbol{k} \in \widehat{\mathbb{Z}}^{d}} \nu_{\boldsymbol{y}}\left(\left\{M \in G_{\boldsymbol{k}, \boldsymbol{y}} \cap \mathcal{F}_{d}: \mathbb{Z}^{d} M \cap \mathfrak{Z}=\emptyset\right\}\right)
$$

where $\mathfrak{Z}=\xi^{\frac{1}{d}}(\mathfrak{Z}(0,1,1)+(0, \boldsymbol{z})), \boldsymbol{y}=\xi^{\frac{1}{d}}(1, \boldsymbol{z}+\boldsymbol{w}), \widehat{\mathbb{Z}}^{d}$ is the set of primitive vectors in $\mathbb{Z}^{d}$, and $G_{\boldsymbol{k}, \boldsymbol{y}}=\{M \in G: \boldsymbol{k} M=\boldsymbol{y}\}$. Using the bound [29, Prop. 7.3] on the contribution from 
all $\boldsymbol{k}$ with $k_{1} \neq 1$ in (5.2), we get

$$
\Phi_{\mathbf{0}}(\xi, \boldsymbol{w}, \boldsymbol{z})=\sum_{\boldsymbol{k}^{\prime} \in \mathbb{Z}^{d-1}} \nu_{\boldsymbol{y}}\left(\left\{M \in G_{\boldsymbol{k}, \boldsymbol{y}} \cap \mathcal{F}_{d}: \mathbb{Z}^{d} M \cap \mathfrak{Z}=\emptyset\right\}\right)+O\left(E_{1}\right),
$$

where we write $\boldsymbol{k}=\left(1, \boldsymbol{k}^{\prime}\right)$, and where

$$
E_{1}:= \begin{cases}\xi^{-2} \log \left(2+\min \left(\xi, \varphi^{-1}\right)\right) & \text { if } d=3 \\ \xi^{-2} \min \left(1,\left(\xi \varphi^{d-2}\right)^{-\frac{d-3}{d-1}}\right) & \text { if } d \geq 4\end{cases}
$$

Using now Lemma 3.4 and the fact that $a_{1}>A$ holds for all $M \in \mathcal{F}_{d}$ with $\mathbb{Z}^{d} M \cap \mathfrak{Z}=\emptyset$, we get:

$\Phi_{\mathbf{0}}(\xi, \boldsymbol{w}, \boldsymbol{z})=\sum_{\boldsymbol{k}^{\prime} \in \mathbb{Z}^{d-1}} \nu_{\boldsymbol{y}}\left(\left\{M \in G_{\boldsymbol{k}, \boldsymbol{y}} \cap \mathcal{G}_{A}: \mathbb{Z}^{d} M \cap \mathfrak{Z}=\emptyset\right\}\right)$

$$
+O\left(\sum_{\boldsymbol{k}^{\prime} \in \mathbb{Z}^{d-1}} \nu_{\boldsymbol{y}}\left(\left\{M \in G_{\boldsymbol{k}, \boldsymbol{y}} \cap\left(\mathcal{S}_{d}^{\prime} \cup \mathcal{G}_{A}\right): \mathbb{Z}^{d} M \cap \mathfrak{Z}=\emptyset, a_{2} \geq\left(c_{1}^{(d-1)}\right)^{-1} A\right\}\right)\right)+O\left(E_{1}\right) .
$$

Following [29], we parametrize $G_{\boldsymbol{k}, \boldsymbol{y}}$ (for $\left.\boldsymbol{k}=\left(1, \boldsymbol{k}^{\prime}\right)\right)$ as follows. For any $\underset{\sim}{M}=\mathrm{n}(\underset{\sim}{u}) \mathrm{a}(\underset{\sim}{a}) \underset{\sim}{\mathbf{k}} \in$ $G^{(d-1)}$ and $\boldsymbol{v} \in \mathrm{S}_{1}^{d-1}$ with $\boldsymbol{y} \cdot \boldsymbol{v}>0$ there is a unique choice of $a_{1}>0, \boldsymbol{u} \in \mathbb{R}^{d-1}$ such that $\left[a_{1}, \boldsymbol{v}, \boldsymbol{u}, \underset{\sim}{\sim}\right] \in G_{\boldsymbol{k}, \boldsymbol{y}}$, namely:

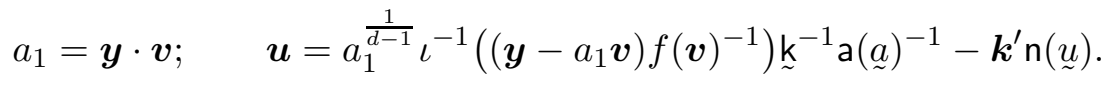

We write $[\boldsymbol{v}, \underset{\sim}{M}]_{\boldsymbol{k}, \boldsymbol{y}}$ for this element $\left[a_{1}, \boldsymbol{v}, \boldsymbol{u}, \underset{\sim}{M}\right] \in G_{\boldsymbol{k}, \boldsymbol{y}}$. This gives a bijective map

$$
\left\{\boldsymbol{v} \in \mathrm{S}_{1}^{d-1}: \boldsymbol{y} \cdot \boldsymbol{v}>0\right\} \times G^{(d-1)} \ni\langle\boldsymbol{v}, \underset{\sim}{M}\rangle \mapsto[\boldsymbol{v}, \underset{\sim}{M}]_{\boldsymbol{k}, \boldsymbol{y}} \in G_{\boldsymbol{k}, \boldsymbol{y}} .
$$

Let $L_{\boldsymbol{v}, M}$ be the lattice

$$
L_{\boldsymbol{v}, M}:=\mathbb{Z}^{d}[\boldsymbol{v}, \underset{\sim}{\sim}]_{\boldsymbol{k}, \boldsymbol{y}}
$$

For any given $\boldsymbol{v}, \underset{\sim}{M}$ as above, $L_{\boldsymbol{v}, M}$ is in fact independent of $\boldsymbol{k}^{\prime} \in \mathbb{Z}^{d-1}$ (for note that $a_{1}, \boldsymbol{v}, \underset{\sim}{\sim}$ are independent of $\boldsymbol{k}^{\prime}$, and so is the congruence class of $\boldsymbol{u} \bmod \mathbb{Z}^{d-1} \mathrm{n}(\underset{u}{u})$; hence the claim follows from $M=\mathrm{n}(u) \mathrm{a}(a) \mathrm{k}$ and (3.9)). Note also that for any $\boldsymbol{v}, M$ as above there is a unique choice of $\boldsymbol{k}^{\prime} \in \mathbb{Z}^{d-1}$ which yields $\boldsymbol{u} \in\left(-\frac{1}{2}, \frac{1}{2}\right]^{d-1}$. Using now the definition of $\mathcal{G}_{A}$ (cf. (3.15) $), a_{2}=a_{1}^{-\frac{1}{d-1}} a_{\sim}$, and the expression for the measure $\nu_{\boldsymbol{y}}$ in the parameters $\boldsymbol{v}, \underset{\sim}{\sim}([29$, Lemma 5.2]), we conclude

$$
\begin{aligned}
& \Phi_{\mathbf{0}}(\xi, \boldsymbol{w}, \boldsymbol{z})=\zeta(d)^{-1} \int_{S} \mu^{(d-1)}\left(\left\{\underset{\sim}{M} \in \mathcal{F}_{d-1}: L_{\boldsymbol{v}, M} \cap \mathfrak{Z}=\emptyset\right\}\right) \frac{d \boldsymbol{v}}{(\boldsymbol{y} \cdot \boldsymbol{v})^{d}} \\
& \quad+O\left(A^{-d} \int_{S} \mu^{(d-1)}\left(\left\{\underset{\sim}{M} \in \mathcal{S}_{d-1}: L_{\boldsymbol{v}, M} \cap \mathfrak{Z}=\emptyset, a_{\sim} \geq\left(c_{1}^{(d-1)}\right)^{-1} A^{\frac{d}{d-1}}\right\}\right) d \boldsymbol{v}\right)+O\left(E_{1}\right),
\end{aligned}
$$

where

$$
S=\left\{\boldsymbol{v} \in \mathrm{S}_{1}^{\prime d-1} \backslash\left\{\boldsymbol{e}_{1}\right\}: a_{1}=\boldsymbol{y} \cdot \boldsymbol{v}>A\right\} .
$$

Here the first error term is

$$
\ll A^{-d} \int_{S} \mu^{(d-1)}\left(\left\{\underset{\sim}{M} \in \mathcal{S}_{d-1}: \mathbb{Z}^{d-1} \underset{\sim}{M} a_{1}^{\frac{1}{d-1}} \mathfrak{Z} \boldsymbol{v}=\emptyset, a_{1} \geq\left(c_{1}^{(d-1)}\right)^{-1} A^{\frac{d}{d-1}}\right\}\right) d \boldsymbol{v},
$$

where $\mathfrak{Z}_{\boldsymbol{v}}=\iota^{-1}\left(\mathfrak{Z} f(\boldsymbol{v})^{-1}\right)$. (For note that $L_{\boldsymbol{v}, M} \cap \mathfrak{Z}=\emptyset$ implies $a_{1}^{-\frac{1}{d-1}} \iota\left(\mathbb{Z}^{d-1} \underset{\sim}{\mathcal{M}}\right) f(\boldsymbol{v}) \cap \mathfrak{Z}=\emptyset$, i.e. $\mathbb{Z}^{d-1} \underset{\sim}{\sim} \cap a_{1}^{\frac{1}{d-1}} \mathfrak{Z}_{\boldsymbol{v}}=\emptyset$; cf. (3.13) .) But for each $\boldsymbol{v} \in S$, the set $a_{1}^{\frac{1}{d-1}} \mathfrak{Z} \boldsymbol{v} \subset \mathbb{R}^{d-1}$ contains an open $(d-1)$-dimensional right cone with $\mathbf{0}$ in its base, of radius $\gg A^{\frac{1}{d-1}} \xi^{\frac{1}{d}} \sin \omega_{\boldsymbol{z}} \gg \xi^{\frac{1}{d-1}} \sin \omega_{\boldsymbol{z}}$ and height $\gg \xi^{\frac{1}{d-1}} \sin ^{2} \omega_{\boldsymbol{z}}$, where $\omega_{\boldsymbol{z}}$ is the angle between $\boldsymbol{v}^{\prime}=\left(v_{2}, \ldots, v_{d}\right)$ and $\boldsymbol{z}$ in $\mathbb{R}^{d-1}$, by 
[29, Lemma 7.1]. Hence using [29, Lemma 7.4] and a parametrization similar to (3.37) but rotated to have $\omega_{\boldsymbol{z}}$ in place of $\omega$, we see that (5.10) is

$$
\ll A^{-d} \int_{0}^{\pi / 2} A^{-d}\left(A^{\frac{d}{d-1}} \xi^{\frac{d-2}{d-1}} \omega_{\boldsymbol{z}}^{d-1}\right)^{\frac{2}{d-1}-1} \omega_{\boldsymbol{z}}^{d-3} d \omega_{\boldsymbol{z}} \ll \xi^{-3+\frac{2}{d-1}} \int_{0}^{\pi / 2} d \omega_{\boldsymbol{z}} \ll \xi^{-3+\frac{2}{d-1}} .
$$

Hence, since $\xi^{-3+\frac{2}{d-1}} \ll E_{1}$, we conclude

$$
\Phi_{\mathbf{0}}(\xi, \boldsymbol{w}, \boldsymbol{z})=\zeta(d)^{-1} \int_{S} \mu\left(\left\{\underset{\sim}{M} \in \mathcal{F}_{d-1}: L_{\boldsymbol{v}, M} \cap \mathfrak{Z}=\emptyset\right\}\right) \frac{d \boldsymbol{v}}{(\boldsymbol{y} \cdot \boldsymbol{v})^{d}}+O\left(E_{1}\right) .
$$

Next, by [29, Prop. 7.5], at the cost of an error which is $O\left(E_{1}\right)$ we may restrict the range of integration in (5.11) to the set

$$
S^{\prime}=\left\{\boldsymbol{v} \in \mathrm{S}_{1}^{\prime d-1} \backslash\left\{\boldsymbol{e}_{1}\right\}: a_{1}=\boldsymbol{y} \cdot \boldsymbol{v}>A, v_{1}>c_{15}^{-2}(\varphi+\omega)^{2}\right\},
$$

where $\omega=\varphi\left(\boldsymbol{v}^{\prime}, \boldsymbol{e}_{1}\right)$ as in (3.37). Recall that $c_{15}<\min \left(c_{11}, c_{13}\right) \leq \frac{1}{10}$ (cf. p. 46). As before we write $\boldsymbol{v}^{\prime}=\left(v_{2}, \ldots, v_{d}\right) \in \mathbb{R}^{d-1}$ and $\boldsymbol{v}^{\prime \prime}=\left(v_{3}, \ldots, v_{d}\right) \in \mathbb{R}^{d-2}$; then $\left\|\boldsymbol{v}^{\prime}\right\|=\sin \varpi$ and $\left\|\boldsymbol{v}^{\prime \prime}\right\|=$ $\sin \varpi \sin \omega$ (cf. (3.37)). Note that $\boldsymbol{v} \in S^{\prime}$ forces $\omega<c_{15} \leq \frac{1}{10}$ and thus $v_{2}=\sin \varpi \cos \omega>0$. We also have

$$
\frac{\left\|\boldsymbol{v}^{\prime \prime}\right\|}{\left\|\boldsymbol{v}^{\prime}\right\|}=\sin \omega<c_{15} \leq \frac{1}{10}, \quad \forall \boldsymbol{v} \in S^{\prime}
$$

and thus also

$$
\left\|\boldsymbol{v}^{\prime \prime}\right\| \leq \frac{1}{10} ; \quad \text { and } \quad v_{1}+2 v_{2} \geq \sqrt{v_{1}^{2}+v_{2}^{2}} \geq \sqrt{\frac{99}{100}}>\frac{9}{10}, \quad \forall \boldsymbol{v} \in S^{\prime} .
$$

We now impose the condition that $c_{15}$ should be so small that (5.1) forces

$$
\left\|\boldsymbol{z}-\boldsymbol{e}_{1}\right\|<\frac{1}{20} \quad \text { and } \quad\left\|\boldsymbol{w}-\boldsymbol{e}_{1}\right\|<\frac{1}{20}
$$

(this is clearly possible, since both $\varphi\left(\boldsymbol{z}, \boldsymbol{e}_{1}\right)$ and $\varphi\left(\boldsymbol{w}, \boldsymbol{e}_{1}\right)$ are always $\leq \varphi$ in (4.37)). Then also $\left\|\boldsymbol{z}+\boldsymbol{w}-2 \boldsymbol{e}_{1}\right\|<\frac{1}{10}$, and since $\boldsymbol{y}=\xi^{\frac{1}{d}}(1, \boldsymbol{z}+\boldsymbol{w})$ it follows that, for all $\boldsymbol{v} \in S^{\prime}$,

$$
\begin{aligned}
\xi^{-\frac{1}{d}} a_{1}=\xi^{-\frac{1}{d}}(\boldsymbol{y} \cdot \boldsymbol{v})=v_{1}+2 \boldsymbol{e}_{1} \cdot \boldsymbol{v}^{\prime}+\left(\boldsymbol{z}+\boldsymbol{w}-2 \boldsymbol{e}_{1}\right) \cdot \boldsymbol{v}^{\prime} \\
\geq v_{1}+2 v_{2}-\frac{1}{10}\left\|\boldsymbol{v}^{\prime}\right\|>\frac{9}{10}-\frac{1}{10}=\frac{4}{5} .
\end{aligned}
$$

Note that this was derived without ever using the condition $\boldsymbol{y} \cdot \boldsymbol{v}>A$ in (5.12); hence that condition is in fact redundant (since $A=c_{4} \xi^{\frac{1}{d}}<\frac{1}{2} \xi^{\frac{1}{d}}$ ), i.e. we have

$$
S^{\prime}=\left\{\boldsymbol{v} \in \mathrm{S}_{1}^{\prime{ }^{d-1}}: v_{1}>c_{15}^{-2}(\varphi+\omega)^{2}\right\} \text {. }
$$

Recall that we always have $L_{\boldsymbol{v}, M} \subset \cup_{n \in \mathbb{Z}}\left(n a_{1} \boldsymbol{v}+\boldsymbol{v}^{\perp}\right)$, cf. (3.14). The following lemma implies that $\left(n a_{1} \boldsymbol{v}+\boldsymbol{v}^{\perp}\right) \cap \mathfrak{Z}=\emptyset$ holds for all $\boldsymbol{v} \in S^{\prime}$ and all $n \in \mathbb{Z} \backslash\{0,1\}$.

Lemma 5.1. For any $\boldsymbol{v} \in \mathrm{S}_{1}^{d-1}$ with $0<v_{1}<1$ and $\varphi\left(\boldsymbol{v}^{\prime}, \boldsymbol{e}_{1}\right) \leq \frac{1}{10}$, and for any $\boldsymbol{z}, \boldsymbol{w} \in \mathcal{B}_{1}^{d-1}$ with $\left\|\boldsymbol{z}-\boldsymbol{e}_{1}\right\|<\frac{1}{10},\left\|\boldsymbol{w}-\boldsymbol{e}_{1}\right\|<\frac{1}{10}$,

$$
\left(n(1, \boldsymbol{z}+\boldsymbol{w})+\boldsymbol{v}^{\perp}\right) \cap(\mathfrak{Z}(0,1,1)+(0, \boldsymbol{z}))=\emptyset, \quad \forall n \in \mathbb{Z} \backslash\{0,1\} .
$$

Proof. Since $n(1, \boldsymbol{z}+\boldsymbol{w})+\boldsymbol{v}^{\perp}$ has nonempty intersection with $\mathfrak{Z}(0,1,1)+(0, \boldsymbol{z})$ for $n=0,1$, and $\mathfrak{Z}(0,1,1)+(0, \boldsymbol{z})$ is convex, it suffices to prove that (5.18) holds for $n=-1$ and for $n=2$. We have $\mathfrak{Z}(0,1,1)+(0, \boldsymbol{z})=\mathfrak{Z}^{\prime}+\left(\frac{1}{2}, \boldsymbol{z}\right)$ where $\mathfrak{Z}^{\prime}:=\mathfrak{Z}\left(-\frac{1}{2}, \frac{1}{2}, 1\right)$; hence $\mathfrak{Z}(0,1,1)+(0, \boldsymbol{z})$ has nonempty intersection with $n(1, \boldsymbol{z}+\boldsymbol{w})+\boldsymbol{v}^{\perp}$ if and only if $\mathfrak{Z}^{\prime}$ has nonempty intersection with

$$
n(1, \boldsymbol{z}+\boldsymbol{w})-\left(\frac{1}{2}, \boldsymbol{z}\right)+\boldsymbol{v}^{\perp}=\left(\left(n-\frac{1}{2}\right) v_{1}+(n-1) \boldsymbol{z} \cdot \boldsymbol{v}^{\prime}+n \boldsymbol{w} \cdot \boldsymbol{v}^{\prime}\right) \boldsymbol{v}+\boldsymbol{v}^{\perp} .
$$

Hence by Lemma 3.6 our task is to prove that $\left|\left(n-\frac{1}{2}\right) v_{1}+(n-1) \boldsymbol{z} \cdot \boldsymbol{v}^{\prime}+n \boldsymbol{w} \cdot \boldsymbol{v}^{\prime}\right| \geq \frac{1}{2} v_{1}+\left\|\boldsymbol{v}^{\prime}\right\|$ for $n=-1,2$, i.e. it suffices to prove that

$$
v_{1}+\boldsymbol{z} \cdot \boldsymbol{v}^{\prime}+2 \boldsymbol{w} \cdot \boldsymbol{v}^{\prime} \geq\left\|\boldsymbol{v}^{\prime}\right\| \quad \text { and } \quad v_{1}+2 \boldsymbol{z} \cdot \boldsymbol{v}^{\prime}+\boldsymbol{w} \cdot \boldsymbol{v}^{\prime} \geq\left\|\boldsymbol{v}^{\prime}\right\|
$$


However this is clear since the assumptions of the lemma imply $\varphi\left(\boldsymbol{z}, \boldsymbol{v}^{\prime}\right)<\frac{1}{3} \pi$ and thus $\boldsymbol{z} \cdot \boldsymbol{v}^{\prime}>\frac{1}{2}\|\boldsymbol{z}\|\left\|\boldsymbol{v}^{\prime}\right\|>\frac{9}{20}\left\|\boldsymbol{v}^{\prime}\right\|$, and similarly $\boldsymbol{w} \cdot \boldsymbol{v}^{\prime}>\frac{9}{20}\left\|\boldsymbol{v}^{\prime}\right\|$.

Let us also note that

$$
\boldsymbol{v}^{\perp} \cap L_{\boldsymbol{v}, M}=a_{1}^{-\frac{1}{d-1}} \iota\left(\mathbb{Z}^{d-1} \underset{\sim}{\sim}\right) f(\boldsymbol{v}) \quad \text { and } \quad\left(a_{1} \boldsymbol{v}+\boldsymbol{v}^{\perp}\right) \cap L_{\boldsymbol{v}, M}=\boldsymbol{y}+a_{1}^{-\frac{1}{d-1}} \iota\left(\mathbb{Z}^{d-1} \underset{\sim}{\sim}\right) f(\boldsymbol{v}) .
$$

Indeed these relations follow from (3.13) with $n=0,1$, if we also note that for any $\boldsymbol{k}=\left(1, \boldsymbol{k}^{\prime}\right)$ $\left(\boldsymbol{k}^{\prime} \in \mathbb{Z}^{d-1}\right)$, if $M=\left[\boldsymbol{v}, \underset{\sim}{M_{\boldsymbol{k}, \boldsymbol{y}}}\right.$ then $\left(\boldsymbol{e}_{1}+\iota\left(\mathbb{Z}^{d-1}\right)\right) M=\left(\boldsymbol{k}+\iota\left(\mathbb{Z}^{d-1}\right)\right) M=\boldsymbol{y}+\iota\left(\mathbb{Z}^{d-1}\right) M$, since $M \in G_{\boldsymbol{k}, \boldsymbol{y}}$. Taking also Lemma 5.1 into account we conclude that for any $\boldsymbol{v} \in S^{\prime}$ we have the equivalence:

$$
L_{\boldsymbol{v}, M} \cap \mathfrak{Z}=\emptyset \quad \Longleftrightarrow \quad a_{1}^{-\frac{1}{d-1}} \iota\left(\mathbb{Z}^{d-1} \underset{\sim}{\sim}\right) f(\boldsymbol{v}) \cap(\mathfrak{Z} \cup(\boldsymbol{y}-\mathfrak{Z}))=\emptyset .
$$

Note here that

$$
\boldsymbol{y}-\mathfrak{Z}=\xi^{\frac{1}{d}}(\mathfrak{Z}(0,1,1)+(0, \boldsymbol{w})) .
$$

Hence by the same argument as we used to get (3.56), we have for every $\boldsymbol{v} \in S^{\prime}$ :

$$
\begin{aligned}
& \mu\left(\left\{\underset{\sim}{\sim} \in \mathcal{F}_{d-1}: L_{\boldsymbol{v}, M} \cap \mathfrak{Z}=\emptyset\right\}\right) \\
& \quad \geq \mu\left(\left\{M \in X_{1}^{(d-1)}: \mathbb{Z}^{d-1} M \cap\left(\xi^{\frac{1}{d}} v_{1}^{-\frac{1}{d-1}} a_{1}^{\frac{1}{d-1}}\right) \mathfrak{C}_{\boldsymbol{v}^{\prime}}(\boldsymbol{z}, \boldsymbol{w})=\emptyset\right\}\right) \\
& \quad=\Upsilon\left(\boldsymbol{z}, \boldsymbol{w}, \boldsymbol{v}^{\prime}, \xi^{-1+\frac{1}{d}} v_{1} a_{1}^{-1}\right),
\end{aligned}
$$

cf. (4.35) and (4.36). Furthermore we have equality in (5.22) whenever both $\boldsymbol{v}^{\perp} \cap \mathfrak{Z} \subset\left\{x_{1}<\right.$ $\left.\frac{1}{2} \xi^{\frac{1}{d}}\right\}$ and $\boldsymbol{v}^{\perp} \cap(\boldsymbol{y}-\mathfrak{Z}) \subset\left\{x_{1}<\frac{1}{2} \xi^{\frac{1}{d}}\right\}$ hold.

Now by Lemma 3.10 (applied after appropriate rotations), if $\boldsymbol{v} \in S^{\prime}$ does not satisfy both $\boldsymbol{v}^{\perp} \cap \mathfrak{Z} \subset\left\{x_{1}<\frac{1}{2} \xi^{\frac{1}{d}}\right\}$ and $\boldsymbol{v}^{\perp} \cap(\boldsymbol{y}-\mathfrak{Z}) \subset\left\{x_{1}<\frac{1}{2} \xi^{\frac{1}{d}}\right\}$, then

$$
\frac{\pi}{2}-\varpi \ll 1-z+\omega_{\boldsymbol{z}}^{2} \quad \text { or } \quad \frac{\pi}{2}-\varpi \ll 1-w+\omega_{\boldsymbol{w}}^{2},
$$

where from now on we write $\omega_{\boldsymbol{w}}$ for the angle between $\boldsymbol{v}^{\prime}$ and $\boldsymbol{w}$, and (as before) $\omega_{\boldsymbol{z}}$ for the angle between $\boldsymbol{v}^{\prime}$ and $\boldsymbol{z}$. Since $w \geq z$, we see that (5.23) forces

$$
\frac{\pi}{2}-\varpi \ll \max \left(\omega_{\boldsymbol{z}}, \omega_{\boldsymbol{w}}\right)^{2} \quad \text { or } \quad \frac{\pi}{2}-\varpi \ll 1-z
$$

to hold (the implied constant depends only on $d$ ). But recall the definition of $S^{\prime}$, (5.12), and note that $\frac{\pi}{2}-\varpi \asymp v_{1}$ and $\max \left(\omega_{\boldsymbol{z}}, \omega_{\boldsymbol{w}}\right) \leq \omega+\varphi$, by the triangle inequality in $\mathrm{S}_{1}^{d-2}$. Hence by choosing $c_{15}$ sufficiently small we can ensure that every $\boldsymbol{v} \in S^{\prime}$ which does not satisfy both $\boldsymbol{v}^{\perp} \cap \mathfrak{Z} \subset\left\{x_{1}<\frac{1}{2} \xi^{\frac{1}{d}}\right\}$ and $\boldsymbol{v}^{\perp} \cap(\boldsymbol{y}-\mathfrak{Z}) \subset\left\{x_{1}<\frac{1}{2} \xi^{\frac{1}{d}}\right\}$ must in fact satisfy $\frac{\pi}{2}-\varpi \ll 1-z$. Now the total contribution from these $\boldsymbol{v} \in S^{\prime}$ to the integral in (5.11) can be bounded by following the proof of (1.38) in [29, Sec. 7.2] (the " $\Sigma_{1}$-part", for $\varphi \leq \frac{\pi}{2}$ ), but restricting the integration by $\frac{\pi}{2}-\varpi \ll 1-z$; cf. especially [29, (7.22)-(7.23)]; it follows that this contribution is:

$$
\ll(1-z) \xi^{-2+\frac{2}{d}} \min \left(1,\left(\xi \varphi^{d}\right)^{-1+\frac{2}{d(d-1)}}\right) .
$$

By Proposition 1.10 we know that $\Phi_{0}(\xi, w, z, \varphi)>0$ implies

$$
\max (1-z, 1-w) \ll \xi^{-\frac{2}{d}} \min \left(1,\left(\xi \varphi^{d}\right)^{-\frac{2}{d(d-1)}}\right) .
$$

Hence if $\Phi_{\mathbf{0}}(\xi, \boldsymbol{w}, \boldsymbol{z})>0$ then (5.24) is $\ll E_{2}$, where

$$
E_{2}:=\xi^{-2} \min \left(1,\left(\xi \varphi^{d}\right)^{-1}\right),
$$

and it follows that:

$$
\Phi_{\mathbf{0}}(\xi, \boldsymbol{w}, \boldsymbol{z})=\zeta(d)^{-1} \int_{\boldsymbol{v} \in S^{\prime}} \Upsilon\left(\boldsymbol{z}, \boldsymbol{w}, \boldsymbol{v}^{\prime}, \xi^{-\frac{d-1}{d}} v_{1} a_{1}^{-1}\right) \frac{d \boldsymbol{v}}{(\boldsymbol{y} \cdot \boldsymbol{v})^{d}}+O\left(E_{1}\right)+O\left(E_{2}\right) .
$$

This is in fact true in general, for in the remaining case when $\Phi_{\mathbf{0}}(\xi, \boldsymbol{w}, \boldsymbol{z})=0$, (5.27) is an obvious consequence of (5.11) and the inequality (5.22). (In (5.27) we may note that $E_{2} \ll E_{1}$ 
if $d=3$, but if $d \geq 4$ then $E_{2} \ll E_{1}$ holds if and only if $\varphi \ll \xi^{-\frac{1}{d-2}}$ or $\varphi \gg \xi^{-\frac{1}{2 d-3}}$. However in the end we will just use $E_{1}, E_{2} \ll E$, cf. (1.30).)

Next, using (4.48), (4.49) and their analogues for $\boldsymbol{w}$, we have for every $\boldsymbol{v} \in S^{\prime}$ :

$$
\begin{aligned}
& a_{1}=\boldsymbol{v} \cdot \boldsymbol{y}=\xi^{\frac{1}{d}}\left(v_{1}+2 v_{2}+\right.\left.\left(z_{1}+w_{1}-2\right) v_{2}+\left(z_{2}+w_{2}\right) v_{3}\right) \\
&=\xi^{\frac{1}{d}}\left(v_{1}+2 v_{2}+O\left(1-z+\varphi^{2}+\left\|\boldsymbol{v}^{\prime \prime}\right\| \varphi\right)\right) .
\end{aligned}
$$

Since $v_{1}+2 v_{2}>\frac{9}{10}$ and $a_{1}>\frac{4}{5} \xi^{\frac{1}{d}}$ for all $\boldsymbol{v} \in S^{\prime}$ (cf. (5.14) and (5.16) $)$, (5.28) implies

$$
a_{1}^{-1}=(\boldsymbol{v} \cdot \boldsymbol{y})^{-1}=\xi^{-\frac{1}{d}} \frac{1}{v_{1}+2 v_{2}}\left(1+O\left(1-z+\varphi^{2}+\left\|\boldsymbol{v}^{\prime \prime}\right\| \varphi\right)\right) .
$$

Now because of (5.1), (5.13) and $c_{15}<c_{11}$, Proposition 4.13 can be applied to bound the integrand in (5.27) from above, throughout the range of integration. It follows (using also (5.29) and the monotonicity property of $\Xi$ in its last argument, Lemma 4.7) that there is a constant $c_{16}$ which only depends on $d$ such that

$$
\begin{aligned}
\Phi_{\mathbf{0}}(\xi, \boldsymbol{w}, \boldsymbol{z}) \leq \frac{\xi^{-1}}{\zeta(d)} \int_{\boldsymbol{v} \in S^{\prime}} \Xi\left(\sqrt{\frac{1-w}{1-z}}, \frac{\varphi}{\sqrt{2(1-z)}} ; \boldsymbol{h} ;\right. \\
\left.2^{1-\frac{d}{2}}(1-z)^{-\frac{d}{2}} \xi^{-1} \frac{v_{1}}{v_{1}+2 v_{2}}\left\{1+c_{16}\left(1-z+\varphi^{2}+\frac{\left\|\boldsymbol{v}^{\prime \prime}\right\|^{2}}{\left\|\boldsymbol{v}^{\prime}\right\|^{2}}\right)\right\}\right) \\
\quad \times\left(1+O\left(1-z+\varphi^{2}+\left\|\boldsymbol{v}^{\prime \prime}\right\| \varphi\right)\right) \frac{d \boldsymbol{v}}{\left(v_{1}+2 v_{2}\right)^{d}}+O\left(E_{1}+E_{2}\right),
\end{aligned}
$$

where

$$
\boldsymbol{h}=\left(h_{1}, \ldots, h_{d-1}\right)=\left(\alpha\left(\frac{\left\|\boldsymbol{v}^{\prime \prime}\right\|}{\left\|\boldsymbol{v}^{\prime}\right\|}\right) v_{2}, 2 \beta\left(\frac{\left\|\boldsymbol{v}^{\prime \prime}\right\|}{\left\|\boldsymbol{v}^{\prime}\right\|}\right) v_{2}+v_{3}, v_{4}, \ldots, v_{d}\right),
$$

and where $\alpha, \beta$ are functions in $\mathrm{C}^{1}\left(\left[0, c_{11}\right]\right)$ satisfying (4.71) .

Similarly, by Proposition 4.14, after possibly increasing $c_{16}$ we also have

$$
\begin{aligned}
& \Phi_{\mathbf{0}}(\xi, \boldsymbol{w}, \boldsymbol{z}) \geq \frac{\xi^{-1}}{\zeta(d)} \int_{\boldsymbol{v} \in S^{\prime}} \Xi\left(\sqrt{\frac{1-w}{1-z}}, \frac{\varphi}{\sqrt{2(1-z)}} ; \boldsymbol{h} ;\right. \\
&\left.2^{1-\frac{d}{2}}(1-z)^{-\frac{d}{2}} \xi^{-1} \frac{v_{1}}{v_{1}+2 v_{2}}\left\{1-c_{16}\left(1-z+\varphi^{2}+\left\|\boldsymbol{v}^{\prime \prime}\right\| \varphi\right)\right\}^{+}\right) \\
& \quad \times\left(1-O\left(1-z+\varphi^{2}+\left\|\boldsymbol{v}^{\prime \prime}\right\| \varphi\right)\right) \frac{d \boldsymbol{v}}{\left(v_{1}+2 v_{2}\right)^{d}}-O\left(E_{1}+E_{2}\right),
\end{aligned}
$$

where

$$
\boldsymbol{h}=\left(h_{1}, \ldots, h_{d-1}\right)=\left(\alpha v_{2}, 2 \beta v_{2}+v_{3}, v_{4}, \ldots, v_{d}\right),
$$

where, this time, $\alpha$ and $\beta$ are real numbers satisfying (4.75).

Remark 5.1. In fact the error term " $-O\left(E_{1}+E_{2}\right)$ " in (5.32) may be improved to " $-O\left(\xi^{-3+\frac{2}{d-1}}\right)$ ". Indeed, note that the error term in (5.3) is non-negative; hence by going through the proof of (5.11) but only aiming for a lower bound we obtain

$$
\Phi_{\mathbf{0}}(\xi, \boldsymbol{w}, \boldsymbol{z}) \geq \zeta(d)^{-1} \int_{S} \mu\left(\left\{\underset{\sim}{M} \in \mathcal{F}_{d-1}: L_{\boldsymbol{v}, M} \cap \mathfrak{Z}=\emptyset\right\}\right) \frac{d \boldsymbol{v}}{(\boldsymbol{y} \cdot \boldsymbol{v})^{d}}-O\left(\xi^{-3+\frac{2}{d-1}}\right) .
$$

Hence, using (5.22) we get

$$
\Phi_{\mathbf{0}}(\xi, \boldsymbol{w}, \boldsymbol{z}) \geq \zeta(d)^{-1} \int_{\boldsymbol{v} \in S^{\prime}} \Upsilon\left(\boldsymbol{z}, \boldsymbol{w}, \boldsymbol{v}^{\prime}, \xi^{-\frac{d-1}{d}} v_{1} a_{1}^{-1}\right) \frac{d \boldsymbol{v}}{(\boldsymbol{y} \cdot \boldsymbol{v})^{d}}-O\left(\xi^{-3+\frac{2}{d-1}}\right),
$$

and now our claim follows by applying Proposition 4.14 . 
5.2. The main term. We wish to simplify the integrals in (5.30) and (5.32). We will first discuss (5.30); the treatment of (5.32) is similar but easier, as we explain at the end of this section. To start with, we introduce new variables of integration $\boldsymbol{\eta}=\left(\eta_{1}, \ldots, \eta_{d-1}\right)$ via

$$
\eta_{j}=\frac{2 v_{j+1}}{v_{1}+2 v_{2}} \quad(j=1, \ldots, d-1) .
$$

A quick computation shows that this formula defines a diffeomorphism

$$
\mathrm{S}_{1}^{d-1} \cap\left\{v_{1}, v_{2}>0\right\} \ni \boldsymbol{v} \mapsto \boldsymbol{\eta} \in(0,1) \times \mathbb{R}^{d-2},
$$

with inverse given by

$$
\boldsymbol{v}=\frac{1}{\sqrt{4\left(1-\eta_{1}\right)^{2}+\|\boldsymbol{\eta}\|^{2}}}\left(2\left(1-\eta_{1}\right), \eta_{1}, \eta_{2}, \eta_{3}, \ldots, \eta_{d-1}\right) .
$$

Now $\frac{\left\|\boldsymbol{v}^{\prime \prime}\right\|^{2}}{\left\|\boldsymbol{v}^{\prime}\right\|^{2}}=\frac{\left\|\boldsymbol{\eta}^{\prime}\right\|^{2}}{\|\boldsymbol{\eta}\|^{2}}$ for all $\boldsymbol{v} \in \mathrm{S}_{1}^{d-1} \cap\left\{v_{1}, v_{2}>0\right\}$, where we write $\boldsymbol{\eta}^{\prime}:=\left(\eta_{2}, \ldots, \eta_{d-1}\right)$. Also for all $\boldsymbol{v} \in S^{\prime}$ we have (cf. (5.12), and recall $\boldsymbol{v} \in S^{\prime} \Rightarrow v_{2}>0$ ):

$$
\frac{\left\|\boldsymbol{\eta}^{\prime}\right\|^{2}}{1+\left\|\boldsymbol{\eta}^{\prime}\right\|^{2}}<\frac{\left\|\boldsymbol{\eta}^{\prime}\right\|^{2}}{\|\boldsymbol{\eta}\|^{2}}=\frac{\left\|\boldsymbol{v}^{\prime \prime}\right\|^{2}}{\left\|\boldsymbol{v}^{\prime}\right\|^{2}}<c_{15}^{2} v_{1}<\frac{1-\eta_{1}}{\sqrt{4\left(1-\eta_{1}\right)^{2}+\|\boldsymbol{\eta}\|^{2}}}<\frac{1}{\sqrt{1+\left\|\boldsymbol{\eta}^{\prime}\right\|^{2}}}
$$

and this forces

$$
\left\|\boldsymbol{\eta}^{\prime}\right\|<2
$$

Hence $\sqrt{4\left(1-\eta_{1}\right)^{2}+\|\boldsymbol{\eta}\|^{2}} \asymp 1$ and $v_{1} \asymp 1-\eta_{1}$ and $\left\|\boldsymbol{v}^{\prime \prime}\right\| \asymp\left\|\boldsymbol{\eta}^{\prime}\right\|$ for all $\boldsymbol{v} \in S^{\prime}$.

Also note that for $\boldsymbol{v}, \boldsymbol{\eta}$ related by the above diffeomorphism, we have for any $j \geq 2, \ell \geq 1$ :

$$
\begin{aligned}
& \frac{\partial v_{j}}{\partial \eta_{\ell}}=\frac{\partial}{\partial \eta_{\ell}}\left(\frac{\eta_{j-1}}{\sqrt{4\left(1-\eta_{1}\right)^{2}+\|\boldsymbol{\eta}\|^{2}}}\right)=\frac{\delta_{\ell, j-1}}{\sqrt{4\left(1-\eta_{1}\right)^{2}+\|\boldsymbol{\eta}\|^{2}}}-\frac{\eta_{j-1}}{2\left(4\left(1-\eta_{1}\right)^{2}+\|\boldsymbol{\eta}\|^{2}\right)^{3 / 2}}\left\{\begin{array}{ll}
10 \eta_{1}-8 & \text { if } \ell=1 \\
2 \eta_{\ell} & \text { if } \ell \geq 2
\end{array}\right\} \\
& =\frac{1}{2}\left(v_{1}+2 v_{2}\right)\left(\delta_{\ell, j-1}-v_{j}\left\{\begin{array}{ll}
v_{2}-2 v_{1} & \text { if } \ell=1 \\
v_{\ell+1} & \text { if } \ell \geq 2
\end{array}\right\}\right) .
\end{aligned}
$$

Hence for $\boldsymbol{v} \in S^{\prime}$ we have, using also (5.14) and $\frac{\partial v_{2}}{\partial \eta_{1}}=\frac{1}{2}\left(v_{1}+2 v_{2}\right)^{2} v_{1}\left(1+\frac{\left\|\boldsymbol{v}^{\prime \prime}\right\|^{2}}{v_{1}\left(v_{1}+2 v_{2}\right)}\right)$ :

$$
\frac{\partial v_{j}}{\partial \eta_{\ell}}= \begin{cases}\frac{1}{2}\left(v_{1}+2 v_{2}\right)^{2} v_{1}\left(1+O\left(v_{1}^{-1}\left\|\boldsymbol{v}^{\prime \prime}\right\|^{2}\right)\right) & \text { if } j=2, \ell=1 \\ O\left(\left\|\boldsymbol{v}^{\prime \prime}\right\|\right) & \text { if }[j=2, \ell>1] \text { or }[j>2, \ell=1] \\ \frac{1}{2}\left(v_{1}+2 v_{2}\right)\left(\delta_{\ell, j-1}+O\left(\left\|\boldsymbol{v}^{\prime \prime}\right\|^{2}\right)\right) & \text { if } j>2, \ell>1 .\end{cases}
$$

Hence the Jacobian is:

$$
\frac{\partial\left(v_{2}, \ldots, v_{d}\right)}{\partial\left(\eta_{1}, \ldots, \eta_{d-1}\right)}=2^{1-d}\left(v_{1}+2 v_{2}\right)^{d} v_{1}\left(1+O\left(\frac{\left\|\boldsymbol{v}^{\prime \prime}\right\|^{2}}{v_{1}}\right)\right) .
$$

Finally recall that if we parametrize $\mathrm{S}_{1}^{\prime d-1}$ with $v_{2}, \ldots, v_{d}$ then $d \boldsymbol{v}=v_{1}^{-1} d v_{2} d v_{3} \cdots d v_{d}$.

In view of the above observations, (5.30) now becomes

$$
\begin{aligned}
\Phi_{\mathbf{0}}(\xi, \boldsymbol{w}, \boldsymbol{z}) \leq \frac{2^{1-d} \xi^{-1}}{\zeta(d)} \int_{S^{\prime \prime}} \Xi\left(a, b ; \boldsymbol{h} ; \kappa\left(1-\eta_{1}\right)\left\{1+c_{16}\left(1-z+\varphi^{2}+\frac{\left\|\boldsymbol{\eta}^{\prime}\right\|^{2}}{\|\boldsymbol{\eta}\|^{2}}\right)\right\}\right) \\
\times\left(1+O\left(1-z+\varphi^{2}+\frac{\left\|\boldsymbol{\eta}^{\prime}\right\|^{2}}{1-\eta_{1}}\right)\right) d \boldsymbol{\eta}+O\left(E_{1}+E_{2}\right),
\end{aligned}
$$

where from now on we write

$$
a=\sqrt{\frac{1-w}{1-z}}, \quad b=\frac{\varphi}{\sqrt{2(1-z)}}, \quad \kappa=2^{1-\frac{d}{2}} \xi^{-1}(1-z)^{-\frac{d}{2}},
$$

and where $d \boldsymbol{\eta}=d \eta_{1} \cdots d \eta_{d-1}$, and $S^{\prime \prime}$ is the set of all $\boldsymbol{\eta} \in(0,1) \times \mathbb{R}^{d-2}$ which correspond to $\boldsymbol{v} \in S^{\prime}$ under our diffeomorphism. Let us write $\alpha=\alpha\left(\frac{\left\|\boldsymbol{\eta}^{\prime}\right\|}{\|\boldsymbol{\eta}\|}\right)$ and $\beta=\beta\left(\frac{\left\|\boldsymbol{\eta}^{\prime}\right\|}{\|\boldsymbol{\eta}\|}\right)$ for short, 
and recall (5.31). Also recall that the dependence of $\Xi$ on its third argument is only up to proportionality. Hence in (5.36) we may take

$$
\boldsymbol{h}=\left(\eta_{1}, \alpha^{-1}\left(2 \beta \eta_{1}+\eta_{2}\right), \alpha^{-1} \eta_{3}, \ldots, \alpha^{-1} \eta_{d-1}\right) .
$$

We will next carry out one more substitution, taking $h_{1}, \ldots, h_{d-1}$ as new variables of integration. Note that (5.38) defines a $\mathrm{C}^{1}$ function $\boldsymbol{\eta} \mapsto \boldsymbol{h}$ for all $\boldsymbol{\eta}$ in the open cone

$$
\Omega=\left\{\boldsymbol{\eta} \in \mathbb{R}^{d-1}: 0<\eta_{1}<1,\left\|\boldsymbol{\eta}^{\prime}\right\|<\left(c_{15}^{-2}-1\right)^{-\frac{1}{2}} \eta_{1}\right\}
$$

(since $\left.\left\|\boldsymbol{\eta}^{\prime}\right\|<\left(c_{15}^{-2}-1\right)^{-\frac{1}{2}} \eta_{1} \Rightarrow \frac{\left\|\boldsymbol{\eta}^{\prime}\right\|}{\|\boldsymbol{\eta}\|}<c_{15}<c_{11}\right)$. It follows from (15.13) that $S^{\prime \prime} \subset \Omega$. We have

$$
\frac{\partial}{\partial \eta_{1}}\left(\frac{\left\|\boldsymbol{\eta}^{\prime}\right\|}{\|\boldsymbol{\eta}\|}\right)=-\frac{\eta_{1}\left\|\boldsymbol{\eta}^{\prime}\right\|}{\|\boldsymbol{\eta}\|^{3}} \quad \text { and } \quad \frac{\partial}{\partial \eta_{j}}\left(\frac{\left\|\boldsymbol{\eta}^{\prime}\right\|}{\|\boldsymbol{\eta}\|}\right)=\frac{\eta_{1}^{2} \eta_{j}}{\|\boldsymbol{\eta}\|^{3}\left\|\boldsymbol{\eta}^{\prime}\right\|} \quad(j \geq 2) .
$$

Hence for all $\boldsymbol{\eta} \in \Omega$ we have, using also (4.71) and $\|\boldsymbol{\eta}\| \ll \eta_{1}$ :

$$
\begin{array}{lll}
\left|\frac{\partial}{\partial \eta_{1}}\left(\alpha\left(\frac{\left\|\boldsymbol{\eta}^{\prime}\right\|}{\|\boldsymbol{\eta}\|}\right)^{-1}\right)\right| \ll(1-z)^{-\frac{1}{2}}\left(1-z+\varphi^{2}+\frac{\left\|\boldsymbol{\eta}^{\prime}\right\|^{2}}{\|\boldsymbol{\eta}\|^{2}}\right)^{\frac{1}{2}} \frac{\left\|\boldsymbol{\eta}^{\prime}\right\|}{\|\boldsymbol{\eta}\|^{2}} ; & \\
\left|\frac{\partial}{\partial \eta_{j}}\left(\alpha\left(\frac{\left\|\boldsymbol{\eta}^{\prime}\right\|}{\|\boldsymbol{\eta}\|}\right)^{-1}\right)\right| \ll(1-z)^{-\frac{1}{2}}\left(1-z+\varphi^{2}+\frac{\left\|\boldsymbol{\eta}^{\prime}\right\|^{2}}{\|\boldsymbol{\eta}\|^{2}}\right)^{\frac{1}{2}} \frac{1}{\|\boldsymbol{\eta}\|} & (\forall j \geq 2) ; \\
\left|\frac{\partial}{\partial \eta_{j}}\left(\beta\left(\frac{\left\|\boldsymbol{\eta}^{\prime}\right\|}{\|\boldsymbol{\eta}\|}\right)\right)\right| \ll\left(1-z+\varphi^{2}+\frac{\left\|\boldsymbol{\eta}^{\prime}\right\|^{2}}{\|\boldsymbol{\eta}\|^{2}}\right) \frac{1}{\|\boldsymbol{\eta}\|} & (\forall j \geq 2) .
\end{array}
$$

Using these bounds and (4.71) we obtain, for all $\boldsymbol{\eta} \in \Omega$ :

$$
\frac{\partial h_{k}}{\partial \eta_{j}}=\sqrt{\frac{2}{1-z}}\left(\delta_{k j}+O\left(1-z+\varphi^{2}+\frac{\left\|\boldsymbol{\eta}^{\prime}\right\|^{2}}{\|\boldsymbol{\eta}\|^{2}}\right)\right) \quad(\forall k, j \geq 2) .
$$

It follows that if $1-z+\varphi^{2}+\sup _{\boldsymbol{\eta} \in \Omega}\left(\frac{\left\|\boldsymbol{\eta}^{\prime}\right\|^{2}}{\|\boldsymbol{\eta}\|^{2}}\right)$ is sufficiently small then our map $\boldsymbol{\eta} \mapsto \boldsymbol{h}$ is injective on all of $\Omega$. Because of (5.1) and (5.39) we can ensure this by requiring the constant $c_{15}$ to be sufficiently small. In particular it now follows that the map $\boldsymbol{\eta} \mapsto \boldsymbol{h}$ restricts to a diffeomorphism from $S^{\prime \prime}$ onto some open set $S^{\prime \prime \prime} \subset(0,1) \times \mathbb{R}^{d-2}$.

Using (5.40) together with $\frac{\partial h_{1}}{\partial \eta_{j}}=\delta_{1 j}$ we get

$$
\frac{\partial\left(h_{1}, \ldots, h_{d-1}\right)}{\partial\left(\eta_{1}, \ldots, \eta_{d-1}\right)}=\left(\frac{2}{1-z}\right)^{\frac{d}{2}-1}\left(1+O\left(1-z+\varphi^{2}+\frac{\left\|\boldsymbol{\eta}^{\prime}\right\|^{2}}{\|\boldsymbol{\eta}\|^{2}}\right)\right) .
$$

By requiring $c_{15}$ to be sufficiently small we may assume that the big- $O$ term in (5.41) is $<\frac{1}{2}$, say. We now obtain from (5.36):

$$
\begin{array}{r}
\Phi_{\mathbf{0}}(\xi, \boldsymbol{w}, \boldsymbol{z}) \leq \frac{2^{2-\frac{3}{2} d} \xi^{-1}(1-z)^{\frac{d}{2}-1}}{\zeta(d)} \int_{S^{\prime \prime \prime}} \Xi\left(a, b ; \boldsymbol{h} ; \kappa\left(1-h_{1}\right)\left\{1+c_{16}\left(1-z+\varphi^{2}+\frac{\left\|\boldsymbol{\eta}^{\prime}\right\|^{2}}{\|\boldsymbol{\eta}\|^{2}}\right)\right\}\right) \\
\times\left(1+O\left(1-z+\varphi^{2}+\frac{\left\|\boldsymbol{\eta}^{\prime}\right\|^{2}}{1-\eta_{1}}+\frac{\left\|\boldsymbol{\eta}^{\prime}\right\|^{2}}{\|\boldsymbol{\eta}\|^{2}}\right)\right) d \boldsymbol{h}+O\left(E_{1}+E_{2}\right) .
\end{array}
$$

The treatment of (5.32) is quite similar: By exactly the same argument as that leading to (5.36) we obtain, after possibly increasing $c_{16}$,

$$
\begin{aligned}
\Phi_{\mathbf{0}}(\xi, \boldsymbol{w}, \boldsymbol{z}) \geq \frac{2^{1-d} \xi^{-1}}{\zeta(d)} \int_{S^{\prime \prime}} \Xi(a, b ; \boldsymbol{h} ; \kappa(1 & \left.\left.-\eta_{1}\right)\left(1-c_{16}\left(1-z+\varphi^{2}+\left\|\boldsymbol{\eta}^{\prime}\right\| \varphi\right)\right)^{+}\right) \\
& \times\left(1-O\left(1-z+\varphi^{2}+\frac{\left\|\boldsymbol{\eta}^{\prime}\right\|^{2}}{1-\eta_{1}}\right)\right) d \boldsymbol{\eta}-O\left(E_{1}+E_{2}\right),
\end{aligned}
$$

where $S^{\prime \prime}$ is the same as in (5.36), and $\boldsymbol{h}$ is as in (5.38), where this time $\alpha, \beta$ are real numbers satisfying (4.75). Just as before we now carry out one more substitution, taking $h_{1}, \ldots, h_{d-1}$ 
as new variables of integration. Since $\alpha, \beta$ are now constants independent of $\boldsymbol{\eta}$, this transformation is much less complicated than before; in fact it is just a (non-singular) linear map $\mathbb{R}^{d-1} \ni \boldsymbol{\eta} \mapsto \boldsymbol{h} \in \mathbb{R}^{d-1}$, with Jacobian

$$
\frac{\partial\left(h_{1}, \ldots, h_{d-1}\right)}{\partial\left(\eta_{1}, \ldots, \eta_{d-1}\right)}=\alpha^{2-d}=\left(\frac{2}{1-z}\right)^{\frac{d}{2}-1}\left(1+O\left(1-z+\varphi^{2}\right)\right)
$$

(cf. (4.75) ). Hence we get

$$
\begin{aligned}
& \Phi_{\mathbf{0}}(\xi, \boldsymbol{w}, \boldsymbol{z}) \geq \frac{2^{2-\frac{3}{2} d} \xi^{-1}(1-z)^{\frac{d}{2}-1}}{\zeta(d)} \int_{S^{\prime \prime \prime}} \Xi\left(a, b ; \boldsymbol{h} ; \kappa\left(1-h_{1}\right)\left(1-c_{16}\left(1-z+\varphi^{2}+\left\|\boldsymbol{\eta}^{\prime}\right\| \varphi\right)\right)^{+}\right) \\
& \times(5.44) \quad \times\left(1-O\left(1-z+\varphi^{2}+\frac{\left\|\boldsymbol{\eta}^{\prime}\right\|^{2}}{1-\eta_{1}}\right)\right) d \boldsymbol{h}-O\left(E_{1}+E_{2}\right),
\end{aligned}
$$

where (again) $S^{\prime \prime \prime} \subset(0,1) \times \mathbb{R}^{d-2}$ is the image of $S^{\prime \prime}$ under our (this time linear) map $\boldsymbol{\eta} \mapsto \boldsymbol{h}$.

5.3. Bounds on some integrals involving $\Xi$. In order to bound the contribution from the various error terms in (5.42) we first prove some auxiliary bounds on integrals involving the $\Xi$-function.

Lemma 5.2. We have, uniformly over all $a>0, b \geq 0, v>0$,

$$
\int_{\mathbb{R}^{d-2}} \Xi(a, b ;(1, \boldsymbol{u}) ; v) d \boldsymbol{u} \ll \min \left\{v^{1-\frac{2}{d}}, v^{2-\frac{2}{d-1}} b^{-d+\frac{2}{d-1}}\right\} .
$$

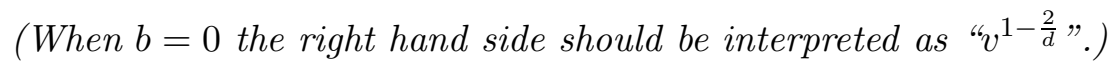

Remark 5.2. Note that $v^{1-\frac{2}{d}} \leq v^{2-\frac{2}{d-1}} b^{-d+\frac{2}{d-1}}$ if and only if $b \leq v^{\frac{1}{d}}$.

Proof. By Lemma 4.5 we have

$$
\Xi(a, b ;(1, \boldsymbol{u}) ; v) \ll \min \left\{1,\left(\left(\max \left(u_{1}^{2},\left(2 b+u_{1}\right)^{2}\right)+u_{2}^{2}+\ldots+u_{d-2}^{2}\right)^{-\frac{d}{2}} v\right)^{2-\frac{2}{d-1}}\right\} .
$$

Let us first assume $d \geq 4$. By noting the symmetry $u_{1} \leftrightarrow-2 b-u_{1}$, and using polar coordinates for the remaining variables $\left(u_{2}, \ldots, u_{d-2}\right)=r \boldsymbol{\omega}\left(\boldsymbol{\omega} \in \mathrm{S}_{1}^{d-4}\right)$, we get

$$
\begin{aligned}
& \int_{\mathbb{R}^{d-2}} \Xi(a, b ;(1, \boldsymbol{u}) ; v) d \boldsymbol{u} \ll \int_{b}^{\infty} \int_{0}^{\infty} \min \left\{1,\left(\left(u_{1}^{2}+r^{2}\right)^{-\frac{d}{2}} v\right)^{2-\frac{2}{d-1}}\right\} r^{d-4} d r d u_{1} \\
& \ll \int_{0}^{\infty} \int_{r+b}^{\infty} \min \left\{1,\left(x^{-d} v\right)^{2-\frac{2}{d-1}}\right\} d x r^{d-4} d r \\
& \ll \int_{0}^{\infty} v^{\frac{1}{d}} \min \left\{1,\left((r+b) v^{-\frac{1}{d}}\right)^{-\frac{2 d^{2}-5 d+1}{d-1}}\right\} r^{d-4} d r .
\end{aligned}
$$

This is always

$$
\begin{aligned}
& \ll v^{\frac{1}{d}} \int_{0}^{\infty}\left((r+b) v^{-\frac{1}{d}}\right)^{-\frac{2 d^{2}-5 d+1}{d-1}} r^{d-4} d r \\
& \ll v^{2-\frac{2}{d-1}}\left(\int_{0}^{b} b^{-\frac{2 d^{2}-5 d+1}{d-1}} r^{d-4} d r+\int_{b}^{\infty} r^{-\frac{2 d^{2}-5 d+1}{d-1}+d-4} d r\right) \ll v^{2-\frac{2}{d-1}} b^{-d+\frac{2}{d-1}},
\end{aligned}
$$

where we used $-\frac{2 d^{2}-5 d+1}{d-1}+d-3=-d+\frac{2}{d-1}<0$ in the last step. On the other hand if $b<v^{\frac{1}{d}}$ then we can do better as follows:

$$
\ll v^{\frac{1}{d}} \int_{0}^{v^{\frac{1}{d}}} r^{d-4} d r+v^{2-\frac{2}{d-1}} \int_{v^{\frac{1}{d}}}^{\infty} r^{-\frac{2 d^{2}-5 d+1}{d-1}+d-4} d r \ll v^{1-\frac{2}{d}}
$$

In the remaining case $d=3$ we get instead

$$
\int_{\mathbb{R}^{d-2}} \Xi(a, b ;(1, \boldsymbol{u}) ; v) d \boldsymbol{u} \ll \int_{b}^{\infty} \min \left\{1, u_{1}^{-3} v\right\} d u_{1} \ll \min \left(v^{\frac{1}{3}}, v b^{-2}\right),
$$

which again agrees with (5.45). 
Lemma 5.3. We have, uniformly over all $a>0, b \geq 0, v>0$,

$$
\int_{\boldsymbol{h} \in(0,1) \times \mathbb{R}^{d-2}} \Xi\left(a, b ; \boldsymbol{h} ;\left(1-h_{1}\right) v\right) d \boldsymbol{h} \ll \min \left\{v^{1-\frac{2}{d}}, v^{2-\frac{2}{d-1}} b^{-d+\frac{2}{d-1}}\right\} .
$$

Proof. Writing $\boldsymbol{h}=h_{1}(1, \boldsymbol{u})$ we get

$$
\int_{\boldsymbol{h} \in(0,1) \times \mathbb{R}^{d-2}} \Xi\left(a, b ; \boldsymbol{h} ;\left(1-h_{1}\right) v\right) d \boldsymbol{h}=\int_{0}^{1} h_{1}^{d-2} \int_{\mathbb{R}^{d-2}} \Xi\left(a, b ;(1, \boldsymbol{u}) ;\left(1-h_{1}\right) v\right) d \boldsymbol{u} d h_{1} .
$$

Hence the lemma follows from Lemma 5.2 ,

Lemma 5.4. We have, uniformly over all $a>0, b \geq 0, v \geq 1,0 \leq \rho \leq \min \left(b^{-2}, v^{-\frac{2}{d}}\right)$,

$$
\begin{aligned}
\int_{(0,1) \times \mathbb{R}^{d-2}} & \Xi\left(a, b ; \boldsymbol{h} ; v\left(1-h_{1}\right)\right) \min \left(1, \rho \frac{\left(b h_{1}\right)^{2}+\|\boldsymbol{h}\|^{2}}{h_{1}^{2}\left(1-h_{1}\right)}\right) d \boldsymbol{h} \\
& \ll \begin{cases}\rho v \log \left(2+\rho^{-1} \min \left(b^{-2}, v^{-2 / 3}\right)\right) & \text { if } d=3 \\
\rho v \min \left(1,\left(v b^{-d}\right)^{1-\frac{2}{d-1}}\right) & \text { if } d \geq 4 .\end{cases}
\end{aligned}
$$

Proof. We first assume $d \geq 4$. Substituting $\boldsymbol{h}=(1-t)\left(1, u_{1}, r \boldsymbol{\omega}\right)$ with $\boldsymbol{\omega} \in \mathrm{S}_{1}^{d-4}$, and using the bound on $\Xi$ from Lemma 4.5, similarly as in the proof of Lemma 5.2 , we see that the left hand side of $(5.46)$ is

$$
\begin{aligned}
& \ll \int_{0}^{\infty} \int_{-\infty}^{\infty} \int_{0}^{1} \min \left\{1,\left(\left(\max \left(u_{1}^{2},\left(2 b+u_{1}\right)^{2}\right)+r^{2}\right)^{-\frac{d}{2}} v t\right)^{2-\frac{2}{d-1}}\right\} \\
& \times \min \left\{1, \frac{\rho\left(1+b^{2}+u_{1}^{2}+r^{2}\right)}{t}\right\} d t d u_{1} r^{d-4} d r .
\end{aligned}
$$

We now prove an auxiliary result:

Lemma 5.5. For any fixed $\delta>0$ we have, uniformly over all $A>0, B \geq 0$ :

$$
\int_{0}^{1} \min \left\{1, A t^{\delta}\right\} \min \left\{1, \frac{B}{t}\right\} d t \asymp \min (1, A) \min \left\{1, B \log \left(2+\frac{1}{A^{-1}+B}\right)\right\} .
$$

Proof. First assume $A \leq 10^{\delta}$. Then the left hand side of (5.48) is

$$
\asymp A \int_{0}^{1} t^{\delta} \min \left\{1, \frac{B}{t}\right\} d t \asymp A \min (1, B),
$$

i.e. (5.48) holds. Next assume $A>10^{\delta}$. Then the left hand side of (5.48) is

$$
\asymp A \int_{0}^{A^{-1 / \delta}} t^{\delta} \min \left\{1, \frac{B}{t}\right\} d t+\int_{A^{-1 / \delta}}^{1} \min \left\{1, \frac{B}{t}\right\} d t .
$$

If $B \leq A^{-1 / \delta}$ then this is $\asymp B+B \log A \asymp B \log A$; if $A^{-1 / \delta} \leq B \leq \frac{1}{10}$ then it is $\asymp A^{-1 / \delta}+$ $B \log \left(B^{-1}\right) \asymp B \log \left(B^{-1}\right)$, and finally if $B \geq \frac{1}{10}$ then it is $\asymp A^{-1 / \delta}+1 \asymp 1$. Hence (5.48) holds in all cases.

We now continue onwards with the proof of Lemma 5.4. By Lemma 5.5 (used together with $\left.\log \left(2+\frac{1}{A^{-1}+B}\right) \leq \log (2+A)\right),(5.47)$ is

$$
\begin{aligned}
& \ll \int_{0}^{\infty} \int_{-\infty}^{\infty} \min \left\{1,\left(\left(b+\left|u_{1}\right|+r\right)^{-d} v\right)^{2-\frac{2}{d-1}}\right\} \\
& \times \min \left\{1, \rho\left(1+b^{2}+u_{1}^{2}+r^{2}\right) \log \left(2+\left(b+\left|u_{1}\right|+r\right)^{-d} v\right)\right\} d u_{1} r^{d-4} d r .
\end{aligned}
$$

Let us first assume $b \geq 1$. Then $1+b^{2}+u_{1}^{2}+r^{2} \ll\left(b+\left|u_{1}\right|+r\right)^{2}$ for all $u_{1} \in \mathbb{R}$, and we thus get, upon setting $s=b+\left|u_{1}\right|+r$,

$$
\ll \int_{b}^{\infty} \min \left\{1,\left(s^{-d} v\right)^{2-\frac{2}{d-1}}\right\} \min \left\{1, \rho s^{2} \log \left(2+s^{-d} v\right)\right\} s^{d-3} d s .
$$


By our assumptions we have $b \leq \rho^{-\frac{1}{2}}$; hence the above is

$$
\ll \int_{b}^{\rho^{-\frac{1}{2}}} \rho s^{2} \log \left(2+s^{-d} v\right) \min \left\{1,\left(s^{-d} v\right)^{2-\frac{2}{d-1}}\right\} s^{d-3} d s+\int_{\rho^{-\frac{1}{2}}}^{\infty}\left(s^{-d} v\right)^{2-\frac{2}{d-1}} s^{d-3} d s .
$$

Also by our assumptions we have $v^{\frac{1}{d}} \leq \rho^{-\frac{1}{2}}$. Using this and the fact that $d-1-d\left(2-\frac{2}{d-1}\right)<-1$ for $d \geq 4$, we find by a quick computation that the above is

$$
\ll \rho v \min \left(1,\left(v b^{-d}\right)^{1-\frac{2}{d-1}}\right)+v^{2-\frac{2}{d-1}} \rho^{\frac{d}{2}-\frac{1}{d-1}} \ll \rho v \min \left(1,\left(v b^{-d}\right)^{1-\frac{2}{d-1}}\right) .
$$

(The last step follows since $\rho v \geq v^{2-\frac{2}{d-1}} \rho^{\frac{d}{2}-\frac{1}{d-1}} \Leftrightarrow \rho \leq v^{-\frac{2}{d}}$ and $\rho v^{2-\frac{2}{d-1}} b^{-d\left(1-\frac{2}{d-1}\right)} \geq$ $v^{2-\frac{2}{d-1}} \rho^{\frac{d}{2}-\frac{1}{d-1}} \Leftrightarrow \rho \leq b^{-2}$, both of which are true by assumption.)

It now remains to treat the case $b<1$. In this case $1+b^{2}+u_{1}^{2}+r^{2} \ll\left(b+\left|u_{1}\right|+r\right)^{2}$ still holds whenever $\left|u_{1}\right| \geq 1$ or $r \geq 1$, and hence the contribution from all such $\left\langle u_{1}, r\right\rangle$ in (5.49) is still bounded by (5.50), and hence also bounded by (5.51). Hence it only remains to treat the contribution from $\left\langle u_{1}, r\right\rangle$ with $\left|u_{1}\right|<1$ and $r<1$. But for these $\left\langle u_{1}, r\right\rangle$ we have $1+b^{2}+u_{1}^{2}+r^{2} \ll 1$ and hence the contribution from these $\left\langle u_{1}, r\right\rangle$ in (5.49) is

$$
\begin{aligned}
& \ll \int_{0}^{1} \int_{0}^{1} \rho \log \left(2+\left(u_{1}+r\right)^{-d} v\right) d u_{1} r^{d-4} d r \ll \rho \int_{0}^{2} \log \left(2+s^{-d} v\right) s^{d-3} d s \\
& \ll \rho \log (2+v) \ll \rho v .
\end{aligned}
$$

Hence (5.46) holds also when $b<1$.

Finally we treat the case $d=3$. In this case the left hand side of (5.46) is

$$
\ll \int_{-\infty}^{\infty} \int_{0}^{1} \min \left\{1,\left(\max \left(u_{1}^{2},\left(2 b+u_{1}\right)^{2}\right)\right)^{-\frac{3}{2}} v t\right\} \min \left\{1, \frac{\rho\left(1+b^{2}+u_{1}^{2}\right)}{t}\right\} d t d u_{1} .
$$

If $b \geq 1$ then arguing as before we get

$$
\ll \int_{b}^{\infty} \min \left\{1, s^{-3} v\right\} \min \left\{1, \rho s^{2} \log \left(2+s^{-3} v\right)\right\} d s .
$$

This is the same as "(5.50) with $d=3$ ", and the analysis goes through as before except that there is an extra logarithm factor (since $d-1-d\left(2-\frac{2}{d-1}\right)=-1$ for $d=3$ ), and we obtain the bound in (5.46). The extension to the case $b<1$ works as before.

5.4. Proof of Theorem 1.7. We now bound the contribution from the error term in (5.42). First, it follows from Lemma 5.3 that the contribution from " $O\left(1-z+\varphi^{2}\right)$ " in (5.42) is

$$
\ll E_{3}:=\xi^{-2+\frac{2}{d}} \min \left\{1,\left(\xi \varphi^{d}\right)^{-1+\frac{2}{d(d-1)}}\right\}\left(1-z+\varphi^{2}\right) .
$$

We next consider the contribution from $O\left(\frac{\left\|\boldsymbol{\eta}^{\prime}\right\|^{2}}{1-\eta_{1}}+\frac{\left\|\boldsymbol{\eta}^{\prime}\right\|^{2}}{\|\boldsymbol{\eta}\|^{2}}\right)$. Note that $\frac{\left\|\boldsymbol{\eta}^{\prime}\right\|^{2}}{1-\eta_{1}}+\frac{\left\|\boldsymbol{\eta}^{\prime}\right\|^{2}}{\|\boldsymbol{\eta}\|^{2}} \ll \min \left(1, \frac{\left\|\boldsymbol{\eta}^{\prime}\right\|^{2}}{\eta_{1}^{2}\left(1-\eta_{1}\right)}\right)$ for all $\boldsymbol{h} \in S^{\prime \prime \prime}$. Furthermore

$$
\boldsymbol{\eta}=\left(h_{1}, \alpha h_{2}-2 \beta h_{1}, \alpha h_{3}, \ldots, \alpha h_{d-1}\right),
$$

where we recall that $\alpha=\alpha\left(\frac{\left\|\boldsymbol{\eta}^{\prime}\right\|}{\|\boldsymbol{\eta}\|}\right), \beta=\beta\left(\frac{\left\|\boldsymbol{\eta}^{\prime}\right\|}{\|\boldsymbol{\eta}\|}\right)$. Hence

$$
\frac{\left\|\boldsymbol{\eta}^{\prime}\right\|^{2}}{\eta_{1}^{2}\left(1-\eta_{1}\right)}=\frac{\left(\alpha h_{2}-2 \beta h_{1}\right)^{2}+\left(\alpha h_{3}\right)^{2}+\cdots+\left(\alpha h_{d-1}\right)^{2}}{h_{1}^{2}\left(1-h_{1}\right)} \ll \alpha^{2} \frac{\left(\frac{\beta}{\alpha}\right)^{2} h_{1}^{2}+\left\|\boldsymbol{h}^{\prime}\right\|^{2}}{h_{1}^{2}\left(1-h_{1}\right)} .
$$

Here $\alpha^{2} \ll 1-z$ and $\left|\frac{\beta}{\alpha}\right| \ll 1+\frac{\varphi}{\sqrt{1-z}} \ll 1+b$, by (4.71) (also recall (5.37)). Hence if apply Lemma 5.4 with $\rho=1-z$ and an appropriate choice of $v \asymp(1-z)^{-\frac{d}{2}} \xi^{-1}$, using Lemma 4.7. 
then we conclude (since $\left.\xi^{-1}(1-z)^{\frac{d}{2}-1} \rho v \asymp \xi^{-2}\right)$ that the contribution from $O\left(\frac{\left\|\boldsymbol{\eta}^{\prime}\right\|^{2}}{1-\eta_{1}}+\frac{\left\|\boldsymbol{\eta}^{\prime}\right\|^{2}}{\|\boldsymbol{\eta}\|^{2}}\right)$ in (5.42) is

$$
\ll E:= \begin{cases}\xi^{-2} \log \left(2+\min \left(\xi, \varphi^{-1}\right)\right) & \text { if } d=3 \\ \xi^{-2} \min \left(1,\left(\xi \varphi^{d}\right)^{-\frac{d-3}{d-1}}\right) & \text { if } d \geq 4 .\end{cases}
$$

(This is the same $E$ as in (1.30).) One checks by inspection that $E_{1}+E_{2} \ll E$, cf. (5.4), (5.26). Furthermore, using $1-z<c_{3} \xi^{-\frac{2}{d}}$ we see that (cf. (5.53))

$$
E_{3} \ll \xi^{-2} \min \left\{1,\left(\xi \varphi^{d}\right)^{-1+\frac{2}{d(d-1)}}\right\}+\xi^{-2} \min \left\{\left(\xi \varphi^{d}\right)^{\frac{2}{d}},\left(\xi \varphi^{d}\right)^{-\frac{d-3}{d-1}}\right\} \ll E .
$$

Hence we conclude

$$
\begin{aligned}
\Phi_{\mathbf{0}}(\xi, \boldsymbol{w}, \boldsymbol{z}) \leq \frac{2^{2-\frac{3}{2} d} \xi^{-1}(1-z)^{\frac{d}{2}-1}}{\zeta(d)} \int_{S^{\prime \prime \prime}} \Xi\left(a, b ; \boldsymbol{h} ; \kappa\left(1-h_{1}\right)\right. \\
\left.\quad \times\left\{1+c_{16}\left(1-z+\varphi^{2}+\frac{\left\|\boldsymbol{\eta}^{\prime}\right\|^{2}}{\|\boldsymbol{\eta}\|^{2}}\right)\right\}\right) d \boldsymbol{h}+O(E) .
\end{aligned}
$$

Here note that (cf. (5.54))

$$
\begin{aligned}
\frac{\left\|\boldsymbol{\eta}^{\prime}\right\|^{2}}{\|\boldsymbol{\eta}\|^{2}} \leq \alpha^{2} \frac{\left(h_{2}-2(\beta / \alpha) h_{1}\right)^{2}+h_{3}^{2}+\cdots+}{h_{1}^{2}} & \ll h^{2} \frac{(b+1)^{2} h_{1}^{2}+\left\|\boldsymbol{h}^{\prime}\right\|^{2}}{h_{1}^{2}} \\
& \ll 1-z+\varphi^{2}+(1-z) \frac{\left\|\boldsymbol{h}^{\prime}\right\|^{2}}{h_{1}^{2}} .
\end{aligned}
$$

Also recall (5.1) and $\frac{\left\|\boldsymbol{\eta}^{\prime}\right\|}{\|\boldsymbol{\eta}\|}<c_{15}$ (cf. (5.39) $)$. Hence by requiring $c_{15}$ to be sufficiently small we can force $c_{16}\left(1-z+\varphi^{2}+\frac{\left\|\boldsymbol{\eta}^{\prime}\right\|^{2}}{\|\boldsymbol{\eta}\|^{2}}\right) \leq \frac{1}{2}$ to hold for all $\boldsymbol{h} \in S^{\prime \prime \prime}$. Now from (5.58) we see that there is a constant $c_{17}>0$ which only depends on $d$ such that

$$
\Phi_{\mathbf{0}}(\xi, \boldsymbol{w}, \boldsymbol{z}) \leq \frac{2^{2-\frac{3}{2} d} \xi^{-1}(1-z)^{\frac{d}{2}-1}}{\zeta(d)} \int_{S^{\prime \prime \prime}} \Xi\left(a, b ; \boldsymbol{h} ; \kappa\left(1-h_{1}\right)\left\{1+M\left(h_{1}^{-1}\left\|\boldsymbol{h}^{\prime}\right\|\right)\right\}\right) d \boldsymbol{h}+O(E)
$$

where

$$
M(\ell):=\min \left\{\frac{1}{2}, c_{17}\left(\varphi^{2}+(1-z)\left(1+\ell^{2}\right)\right)\right\} .
$$

Recall that $S^{\prime \prime \prime} \subset(0,1) \times \mathbb{R}^{d-2}$; hence the above inequality remains true if we replace the range of integration by $(0,1) \times \mathbb{R}^{d-2}$. Writing $\boldsymbol{h}=h_{1}(1, \boldsymbol{u})$ we thus get

$$
\begin{array}{r}
\Phi_{\mathbf{0}}(\xi, \boldsymbol{w}, \boldsymbol{z}) \leq \frac{2^{2-\frac{3}{2} d} \xi^{-1}(1-z)^{\frac{d}{2}-1}}{\zeta(d)} \int_{\mathbb{R}^{d-2}} \int_{0}^{1} \Xi\left(a, b ;(1, \boldsymbol{u}) ; \kappa\left(1-h_{1}\right)\right. \\
(5.59) \\
\times h_{1}^{d-2} d h_{1} d \boldsymbol{u}+O(E) .
\end{array}
$$

Now in the inner integral in (5.59) we substitute $h_{1}=1-(1-t)(1+M(\|\boldsymbol{u}\|))^{-1}, t \in$ $[-M(\|\boldsymbol{u}\|), 1]$. Using $M(\|\boldsymbol{u}\|) \leq \frac{1}{2}$ and Lemma 4.7 we see that the contribution from $t<0$ is

$$
\begin{aligned}
& \ll \xi^{-1}(1-z)^{\frac{d}{2}-1} \int_{\mathbb{R}^{d-2}} \Xi(a, b ;(1, \boldsymbol{u}) ; 2 \kappa) M(\|\boldsymbol{u}\|) d \boldsymbol{u} \\
& \ll \xi^{-1}(1-z)^{\frac{d}{2}-1} \int_{\left(\frac{1}{4}, \frac{1}{2}\right) \times \mathbb{R}^{d-2}} \Xi\left(a, b ; \boldsymbol{h} ; 4 \kappa\left(1-h_{1}\right)\right) \min \left(1, \varphi^{2}+(1-z) \frac{\|\boldsymbol{h}\|^{2}}{h_{1}^{2}}\right) d \boldsymbol{h} \ll E,
\end{aligned}
$$

where the last bound follows from Lemma 5.4 and Lemma 5.3 . We also have, for all $t \in[0,1]$ :

$$
\left|h_{1}^{d-2}-t^{d-2}\right| \ll\left|h_{1}-t\right| \ll\left|1-(1+M(\|\boldsymbol{u}\|))^{-1}\right| \ll M(\|\boldsymbol{u}\|) .
$$


Hence we obtain

$$
\begin{aligned}
\Phi_{\mathbf{0}}(\xi, \boldsymbol{w}, \boldsymbol{z}) \leq \frac{2^{2-\frac{3}{2} d} \xi^{-1}(1-z)^{\frac{d}{2}-1}}{\zeta(d)} \int_{\mathbb{R}^{d-2}} \int_{0}^{1} \Xi(a, b ;(1, \boldsymbol{u}) ; \kappa(1-t)) \\
\times\left(t^{d-2}+O(M(\|\boldsymbol{u}\|))\right) d t d \boldsymbol{u}+O(E) .
\end{aligned}
$$

Here the contribution from the $M(\|\boldsymbol{u}\|)$-term is again $\ll E$, since it is bounded above by the first line in (5.60). Hence, setting $\boldsymbol{h}=t(1, \boldsymbol{u})$, we have finally proved:

$$
\Phi_{\mathbf{0}}(\xi, \boldsymbol{w}, \boldsymbol{z}) \leq \frac{2^{2-\frac{3}{2} d} \xi^{-1}(1-z)^{\frac{d}{2}-1}}{\zeta(d)} \int_{(0,1) \times \mathbb{R}^{d-2}} \Xi\left(a, b ; \boldsymbol{h} ; \kappa\left(1-h_{1}\right)\right) d \boldsymbol{h}+O(E) .
$$

In a very similar way we also obtain a similar lower bound: First, in almost exactly the same way as we got (5.58) from (5.42), (5.44) leads to

$\Phi_{\mathbf{0}}(\xi, \boldsymbol{w}, \boldsymbol{z}) \geq \frac{2^{2-\frac{3}{2} d} \xi^{-1}(1-z)^{\frac{d}{2}-1}}{\zeta(d)} \int_{S^{\prime \prime \prime}} \Xi\left(a, b ; \boldsymbol{h} ; \kappa\left(1-h_{1}\right)\left(1-c_{16}\left(1-z+\varphi^{2}+\left\|\boldsymbol{\eta}^{\prime}\right\| \varphi\right)\right)^{+}\right) d \boldsymbol{h}$

$$
-O(E) \text {. }
$$

We will prove that the range of integration in (5.62) may be replaced by $(0,1) \times \mathbb{R}^{d-2}$, at the cost of an error which is $\ll E$. Recall that we have a bijection $\boldsymbol{h} \leftrightarrow \boldsymbol{\eta} \leftrightarrow \boldsymbol{v}$ between $\boldsymbol{h} \in$ $(0,1) \times \mathbb{R}^{d-2}$ and $\boldsymbol{v} \in \mathrm{S}_{1}^{d-1} \cap\left\{v_{1}, v_{2}>0\right\}$, and by definition $S^{\prime \prime \prime}$ is the set of all $\boldsymbol{h} \in(0,1) \times \mathbb{R}^{d-2}$ which correspond to $\boldsymbol{v} \in S^{\prime}$. Hence for any $\boldsymbol{h} \in\left((0,1) \times \mathbb{R}^{d-2}\right) \backslash S^{\prime \prime \prime}$ the corresponding vector $\boldsymbol{v}$ has $v_{1} \leq c_{15}^{-2}(\varphi+\omega)^{2}$, cf. (5.17). Using also $v_{1} \asymp \frac{1-\eta_{1}}{\sqrt{1+\left\|\boldsymbol{\eta}^{\prime}\right\|^{2}}}$ and $\omega \ll \sin \omega=\frac{\left\|\boldsymbol{v}^{\prime \prime}\right\|}{\left\|\boldsymbol{v}^{\prime}\right\|}=\frac{\left\|\boldsymbol{\eta}^{\prime}\right\|}{\|\boldsymbol{\eta}\|}$ (cf. $(\underline{5.35})$ ), we see that there is a constant $c_{18}>0$ such that the error caused by replacing $S^{\prime \prime \prime}$ by $(0,1) \times \mathbb{R}^{d-2}$ in (5.62) is

$$
\leq \xi^{-1}(1-z)^{\frac{d}{2}-1} \int_{(0,1) \times \mathbb{R}^{d-2}} \Xi(a, b ; \boldsymbol{h} ; \kappa)\left\{I\left(\frac{\left\|\boldsymbol{\eta}^{\prime}\right\|^{2}}{\|\boldsymbol{\eta}\|^{2}} \frac{\sqrt{1+\left\|\boldsymbol{\eta}^{\prime}\right\|^{2}}}{1-\eta_{1}}>c_{18}\right)\right.
$$

Using $\sqrt{1+\left\|\boldsymbol{\eta}^{\prime}\right\|^{2}} \leq 1+\left\|\boldsymbol{\eta}^{\prime}\right\|^{2} \leq \eta_{1}^{-2}\|\boldsymbol{\eta}\|^{2}$ we get

$$
\ll \xi^{-1}(1-z)^{\frac{d}{2}-1} \int_{(0,1) \times \mathbb{R}^{d-2}} \Xi(a, b ; \boldsymbol{h} ; \kappa)\left\{\min \left(1, \frac{\left\|\boldsymbol{\eta}^{\prime}\right\|^{2}}{\eta_{1}^{2}\left(1-\eta_{1}\right)}\right)+I\left(\frac{\varphi^{2}}{1-\eta_{1}}>\frac{1}{2} c_{18}\right)\right\} d \boldsymbol{h},
$$

where to bound the second indicator function in (15.63) we used the fact that if $\left\|\boldsymbol{\eta}^{\prime}\right\|>1$ then $\frac{\left\|\boldsymbol{\eta}^{\prime}\right\|^{2}}{\eta_{1}^{2}\left(1-\eta_{1}\right)}>1$. The contribution from the "min"-term in (5.64) is seen to be $\ll E$ using Lemma 5.4 and (5.55) with our present constants $\alpha, \beta$, and the contribution from the " $I(\cdot)$ "term in (5.64) is, using $\eta_{1}=h_{1}$ and Lemma 5.2;

$$
\begin{array}{r}
\ll \varphi^{2} \xi^{-1}(1-z)^{\frac{d}{2}-1} \min \left\{\kappa^{1-\frac{2}{d}}, \kappa^{2-\frac{2}{d-1}} b^{-d+\frac{2}{d-1}}\right\} \ll \varphi^{2} \min \left\{\xi^{-2+\frac{2}{d}}, \xi^{-3+\frac{2}{d-1}} \varphi^{-d+\frac{2}{d-1}}\right\} \\
\ll E_{3} \ll E
\end{array}
$$

(cf. (5.53) and (5.57)).

Hence we have proved that the range of integration in (5.62) may indeed be replaced by $(0,1) \times \mathbb{R}^{d-2}$. Now by the same argument as when going from (5.58) to (5.61) we obtain

$$
\Phi_{\mathbf{0}}(\xi, \boldsymbol{w}, \boldsymbol{z}) \geq \frac{2^{2-\frac{3}{2} d} \xi^{-1}(1-z)^{\frac{d}{2}-1}}{\zeta(d)} \int_{(0,1) \times \mathbb{R}^{d-2}} \Xi\left(a, b ; \boldsymbol{h} ; \kappa\left(1-h_{1}\right)\right) d \boldsymbol{h}-O(E) .
$$

Together, (5.61) and (5.65) imply that the relation (1.29) in Theorem 1.7 holds, with

$$
F_{\mathbf{0}, d}\left(t_{1}, t_{2}, \alpha\right)=\frac{2^{2-\frac{3}{2} d} t_{1}^{\frac{d}{2}-1}}{\zeta(d)} \int_{\boldsymbol{h} \in(0,1) \times \mathbb{R}^{d-2}} \Xi\left(t_{1}^{-\frac{1}{2}} t_{2}^{\frac{1}{2}}, \frac{\alpha}{\sqrt{2 t_{1}}} ; \boldsymbol{h} ; 2^{1-\frac{d}{2}} t_{1}^{-\frac{d}{2}}\left(1-h_{1}\right)\right) d \boldsymbol{h} .
$$


The fact that $F_{\mathbf{0}, d}$ is uniformly bounded follows from Lemma 5.3. Furthermore for any $\delta>0$ there exists a bounded set $C \subset \mathbb{R}^{d-2}$ such that for all $\left\langle t_{1}, t_{2}, \alpha\right\rangle \in[\delta, \infty) \times \mathbb{R}_{>0} \times \mathbb{R}_{\geq 0}$ the support of the integrand in (5.66) is contained in $(0,1) \times C$ (this follows from Lemma 3.16, since $\left.\Xi(a, b ; \boldsymbol{h} ; v) \leq \Xi\left(\frac{\left\|\boldsymbol{h}^{\prime}\right\|}{h_{1}}, v\right)\right)$. Hence Lemma 4.8 implies that $F_{\mathbf{0}, d}$ is continuous. This completes the proof of Theorem 1.7 .

\section{ON THE SUPPORT OF $\Phi_{\mathbf{0}}(\xi, \boldsymbol{w}, \boldsymbol{z})$}

6.1. The functions $\sigma_{d}(r, \alpha)$ and $\xi_{0}(w, z, \varphi)$. We continue to keep $d \geq 3$. Recall that we have defined, for $a>0, b \in \mathbb{R}$ (cf. (4.12)):

$$
\rho(a, b)=\inf \left\{v>0: \exists \boldsymbol{h} \in \mathbb{R}_{+}^{d-1}: \Xi(a, b ; \boldsymbol{h} ; v)>0\right\} .
$$

Note that $\rho(a,-b)=\rho(a, b)$, immediately from (4.8) and (4.6). Also by (4.9) we have the symmetry relation

$$
\rho(a, b)=a^{d} \rho\left(a^{-1}, a^{-1} b\right)
$$

It follows from (5.66) that

$$
F_{\mathbf{0}, d}\left(t_{1}, t_{2}, \alpha\right)>0 \Longleftrightarrow \rho\left(t_{1}^{-\frac{1}{2}} t_{2}^{\frac{1}{2}}, \frac{\alpha}{\sqrt{2 t_{1}}}\right)<2^{1-\frac{d}{2}} t_{1}^{-\frac{d}{2}}
$$

In order to express this relation in a slightly cleaner way we introduce the function

$$
\sigma_{d}(r, \alpha):=2^{\frac{4}{d}-2} r \rho\left(r^{\frac{1}{2}}, 2^{-\frac{1}{2}}(r \alpha)^{\frac{1}{4}}\right)^{-\frac{4}{d}} \quad(r>0, \alpha \geq 0) .
$$

Then (6.1) translates into the symmetry relation

$$
\sigma_{d}(r, \alpha)=\sigma_{d}\left(r^{-1}, \alpha\right)
$$

and (6.2) translates into (1.32), i.e.

$$
F_{\mathbf{0}, d}\left(t_{2}, t_{1}, \alpha\right)>0 \Longleftrightarrow t_{1} t_{2}<\sigma_{d}\left(\frac{t_{2}}{t_{1}}, \frac{\alpha^{4}}{t_{1} t_{2}}\right) .
$$

We remark that we will prove below in Corollary 6.9 that $\rho(a, b) \asymp \max (1, b)$ holds uniformly over $0<a \leq 1, b \geq 0$. This translates into the relation

$$
\sigma_{d}(r, \alpha) \asymp r \min \left(1,(r \alpha)^{-\frac{1}{d}}\right), \quad \forall 0<r \leq 1, \alpha \geq 0 .
$$

We next prove the existence of the continuous function $\xi_{0}:[0,1) \times[0,1) \times[0, \pi] \rightarrow \mathbb{R}_{>0}$ as stated in Theorem 1.8, Let us fix any $\boldsymbol{w}, \boldsymbol{z} \in \mathcal{B}_{1}^{d-1}$. Now the function $\xi \mapsto \Phi_{\mathbf{0}}(\xi, \boldsymbol{w}, \boldsymbol{z})$ is continuous, decreasing (cf. [29, Lemma 7.11]), positive for $\xi$ small (e.g. by Theorem 1.1) and vanishing for all sufficiently large $\xi$ (e.g. by [29, Prop. 1.9]); hence there exists a unique number $\xi_{0}>0$ such that $\Phi_{\mathbf{0}}(\xi, \boldsymbol{w}, \boldsymbol{z})>0 \Leftrightarrow \xi<\xi_{0}$. This proves that there exists a unique function $\xi_{0}:[0,1) \times[0,1) \times[0, \pi] \rightarrow \mathbb{R}_{>0}$ such that $\Phi_{0}(\xi, w, z, \varphi)>0 \Leftrightarrow \xi<\xi_{0}(w, z, \varphi)$. Since $\Phi_{\mathbf{0}}(\xi, \boldsymbol{w}, \boldsymbol{z})$ is continuous (jointly in all three variables) it follows that $\xi_{0}(w, z, \varphi)$ is lower semicontinuous in $(w, z, \varphi) \in[0,1) \times[0,1) \times[0, \pi]$. Finally the fact that $\xi_{0}(w, z, \varphi)$ is upper semicontinuous (and hence continuous) follows from the following lemma, which is a slight generalization of [29, Lemma 7.11]:

Lemma 6.1. For any $\boldsymbol{w}, \boldsymbol{z}, \boldsymbol{w}^{\prime}, \boldsymbol{z}^{\prime} \in \mathcal{B}_{1}^{d-1}$ and $\xi, \xi^{\prime}>0, \Phi_{\mathbf{0}}(\xi, \boldsymbol{w}, \boldsymbol{z}) \geq \Phi_{\mathbf{0}}\left(\xi^{\prime}, \boldsymbol{w}^{\prime}, \boldsymbol{z}^{\prime}\right)$ holds whenever $\left\|\boldsymbol{z}^{\prime}-\boldsymbol{z}\right\|<1-\|\boldsymbol{z}\|,\left\|\boldsymbol{w}^{\prime}-\boldsymbol{w}\right\|<1-\|\boldsymbol{w}\|$ and

$$
\xi^{\prime} \geq \max \left(\left(1-\frac{\left\|\boldsymbol{z}^{\prime}-\boldsymbol{z}\right\|}{1-\|\boldsymbol{z}\|}\right)^{1-d},\left(1-\frac{\left\|\boldsymbol{w}^{\prime}-\boldsymbol{w}\right\|}{1-\|\boldsymbol{w}\|}\right)^{1-d}\right) \xi .
$$

Proof. Follow the proof of [29, Lemma 7.11], but replace the matrix $T$ therein by

$$
T=\left(\begin{array}{cc}
\alpha & \xi^{-1}\left(\boldsymbol{w}^{\prime}+\boldsymbol{z}^{\prime}-\alpha^{-\frac{1}{d-1}}(\boldsymbol{w}+\boldsymbol{z})\right) \\
\mathrm{t}_{0} & \alpha^{-\frac{1}{d-1}} 1_{d-1}
\end{array}\right) \in G \quad\left(\alpha=\xi^{\prime} / \xi\right) .
$$


6.2. Bound from below on the support of $\Phi_{\mathbf{0}}$. The following proposition gives one half of Theorem 1.8,

Proposition 6.2. We have

$$
\xi_{0}(w, z, \varphi) \geq 2^{1-\frac{d}{2}}(1-z)^{-\frac{d}{2}} \rho\left(\sqrt{\frac{1-w}{1-z}}, \frac{\varphi}{\sqrt{2(1-z)}}\right)^{-1}\left\{1-O\left(\max (1-z, 1-w)+\varphi^{2}\right)\right\},
$$

uniformly over all $z, w \in[0,1), \varphi \in\left[0, \frac{\pi}{2}\right]$.

The proof depends on the following lemma (with constants $c_{13}, c_{14}$ as in Proposition 4.14).

Lemma 6.3. Let $z, w, \varphi$ be given with $1-c_{13} \leq z \leq w<1$ and $0 \leq \varphi \leq c_{13}$. Then there exist numbers $\alpha, \beta$ satisfying (4.75) and which have the property that for any $\xi>0$ with $\Phi_{\mathbf{0}}(\xi, z, w, \varphi)=0$ and for $\boldsymbol{z}, \boldsymbol{w}$ as in (4.37), we have

$$
\begin{aligned}
\Xi\left(\sqrt{\frac{1-w}{1-z}}, \frac{\varphi}{\sqrt{2(1-z)}} ;\right. & \left(\alpha v_{2}, 2 \beta v_{2}+v_{3}, v_{4}, \ldots, v_{d}\right) ; \\
& \left.\frac{2^{1-\frac{d}{2}}(1-z)^{-\frac{d}{2}} \xi^{-1} v_{1}}{\boldsymbol{v} \cdot(1, \boldsymbol{z}+\boldsymbol{w})}\left\{1-c_{14}\left(1-z+\varphi^{2}\right)\right\}^{+}\right)=0
\end{aligned}
$$

for all $\boldsymbol{v}=\left(v_{1}, \ldots, v_{d}\right) \in \mathrm{S}_{1}^{d-1}$ with $v_{1}>\frac{199}{200}$ and $v_{2}>0$.

Proof. Let $\xi, z, w, \varphi$ be given with $\xi>0,1-c_{13} \leq z \leq w<1$ and $0 \leq \varphi \leq c_{13}$, and assume $\Phi_{\mathbf{0}}(\xi, z, w, \varphi)=0$. Take $\boldsymbol{z}, \boldsymbol{w}$ as in (4.37), and set $\mathfrak{Z}=\xi^{\frac{1}{d}}(\mathfrak{Z}(0,1,1)+(0, \boldsymbol{z}))$ and $\boldsymbol{y}=\xi^{\frac{1}{d}}(1, \boldsymbol{z}+\boldsymbol{w})$ as in the previous section. Then since $G$ is covered by a countable number of $\mathcal{F}_{d}$-translates we must have $\nu_{\boldsymbol{y}}\left(\left\{M \in G_{\boldsymbol{k}, \boldsymbol{y}}: \mathbb{Z}^{d} M \cap \mathfrak{Z}=\emptyset\right\}\right)=0$ for every $\boldsymbol{k} \in \widehat{\mathbb{Z}}^{d}$. In particular this holds for $\boldsymbol{k}=\boldsymbol{e}_{1}$, and recalling the definition of $L_{\boldsymbol{v}, M}$ in (5.7) it follows that $L_{\boldsymbol{v}, M} \cap \mathfrak{Z}=\emptyset$ for almost all $\langle\boldsymbol{v}, \underset{\sim}{M}\rangle \in\left(\mathrm{S}_{1}^{d-1} \cap \mathbb{R}_{\boldsymbol{y}_{+}}^{d}\right) \times G^{(d-1)}$. Let us write $S\left(\frac{199}{200}\right)$ for the set of all $\boldsymbol{v} \in \mathrm{S}_{1}^{d-1}$ with $v_{1}>\frac{199}{200}$ and $v_{2}>0$. Then for every $\boldsymbol{v} \in S\left(\frac{199}{200}\right)$ we have $\left\|\boldsymbol{v}^{\prime}\right\|=\sqrt{1-v_{1}^{2}}<\frac{1}{10}$ so that $\boldsymbol{v} \cdot \boldsymbol{y}>\xi^{\frac{1}{d}}\left(\frac{199}{200}-\frac{1}{5}\right)>0$, and thus we have $L_{\boldsymbol{v}, M} \cap \mathfrak{Z}=\emptyset$ for almost all $\langle\boldsymbol{v}, \underset{\sim}{M}\rangle \in S\left(\frac{199}{200}\right) \times G^{(d-1)}$.

We next note that for every $\boldsymbol{v} \in S\left(\frac{199}{200}\right)$ the conclusion of Lemma 5.1 holds, viz. for all $n \in \mathbb{Z} \backslash\{0,1\}$ we have $\left(n a_{1} \boldsymbol{v}+\boldsymbol{v}^{\perp}\right) \cap \mathfrak{Z}=\emptyset$, with $a_{1}=\boldsymbol{y} \cdot \boldsymbol{v}$. Indeed, as in the proof of that lemma it suffices to check that (5.19) holds, and this is clear since $\left\|\boldsymbol{v}^{\prime}\right\|<\frac{1}{10}$ for $\boldsymbol{v} \in S\left(\frac{199}{200}\right)$. It now follows as in Section 5.1 that (5.22) holds for all $\boldsymbol{v} \in S\left(\frac{199}{200}\right)$. But also, by Proposition 4.14, there exist numbers $\alpha, \beta$ which only depend on $z, w, \varphi$ (and $d$ ) and which satisfy (4.75) , such that $\Upsilon\left(\boldsymbol{z}, \boldsymbol{w}, \boldsymbol{v}^{\prime}, \xi^{-1+\frac{1}{d}} v_{1} a_{1}^{-1}\right)$ is larger than or equal to the left hand side of (6.7) for all $\boldsymbol{v} \in S\left(\frac{199}{200}\right)$.

These observations together imply that the left hand side of (6.7) must vanish for almost all $\boldsymbol{v} \in S\left(\frac{199}{200}\right)$, and thus since $\Xi$ is continuous (Lemma 4.8), it must vanish for all $\boldsymbol{v} \in S\left(\frac{199}{200}\right)$.

Proof of Proposition 6.2. Because of $\xi_{0}(w, z, \varphi)=\xi_{0}(z, w, \varphi)$ and (6.1), we may assume $z \leq w$ without loss of generality. Note that (6.6) is content-free unless both $\varphi$ and $1-z$ are small; we may thus assume $1-c_{13} \leq z \leq w<1$ and $0 \leq \varphi \leq c_{13}$. Given $z, w, \varphi$ we let $\boldsymbol{z}, \boldsymbol{w}$ be the corresponding points as in (4.37). Also let $\alpha, \beta$ be the corresponding numbers as in Lemma 6.3.

Set $a=\sqrt{\frac{1-w}{1-z}} \in(0,1]$ and $b=\frac{\varphi}{\sqrt{2(1-z)}} \in \mathbb{R}_{\geq 0}$. Fix any number $\rho^{\prime}>\rho(a, b)$. Then there is some $\boldsymbol{h}=\left(h_{1}, \ldots, h_{d-1}\right) \in \mathbb{R}_{+}^{d-1}$, which we fix from now on, such that $\Xi(a, b ; \boldsymbol{h} ; v)>0$ for $v=\rho^{\prime}$ and thus for all $v \geq \rho^{\prime}$. Now let $t$ be a small positive parameter which we will later take to tend to 0 , and set $\boldsymbol{v}:=\left(v_{1}, \boldsymbol{v}^{\prime}\right)$ where

$$
\boldsymbol{v}^{\prime}:=t\left(\alpha^{-1} h_{1}, h_{2}-2(\beta / \alpha) h_{1}, h_{3}, \ldots, h_{d-1}\right) ; \quad v_{1}:=\sqrt{1-\left\|\boldsymbol{v}^{\prime}\right\|^{2}} .
$$


Clearly for all sufficiently small $t$ the vector $\boldsymbol{v}$ is well-defined, lies in $\mathrm{S}_{1}^{d-1}$, and has $v_{1}>\frac{199}{200}$ and $v_{2}>0$. Also note that, for all $v \geq \rho^{\prime}$,

$$
\Xi\left(a, b ;\left(\alpha v_{2}, 2 \beta v_{2}+v_{3}, v_{4}, \ldots, v_{d}\right) ; v\right)=\Xi(a, b ; t \boldsymbol{h} ; v)=\Xi(a, b ; \boldsymbol{h} ; v)>0 .
$$

Hence Lemma 6.3 implies that $\Phi_{0}(\xi, z, w, \varphi)>0$ for all $\xi>0$ with

$$
\xi \leq \rho^{\prime-1} \frac{2^{1-\frac{d}{2}}(1-z)^{-\frac{d}{2}} v_{1}}{\boldsymbol{v} \cdot(1, \boldsymbol{z}+\boldsymbol{w})}\left\{1-c_{14}\left(1-z+\varphi^{2}\right)\right\}^{+} .
$$

Letting now $t \rightarrow 0$, and then using the fact that $\rho^{\prime}$ was arbitrary with $\rho^{\prime}>\rho(a, b)$, it follows that $\Phi_{0}(\xi, z, w, \varphi)>0$ for all $\xi>0$ with

$$
\xi<\rho(a, b)^{-1} 2^{1-\frac{d}{2}}(1-z)^{-\frac{d}{2}}\left\{1-c_{14}\left(1-z+\varphi^{2}\right)\right\}^{+} .
$$

This concludes the proof.

Corollary 6.4. We have $\rho(a, b) \gg 1+|b|$, uniformly over all $0<a \leq 1, b \in \mathbb{R}$.

Proof. We may assume $b \geq 0$, since $\rho(a,-b)=\rho(a, b)$. Given any $a \in(0,1]$ and $b \geq 0$ we may find $z \leq w<1$ and $\varphi \geq 0$ satisfying $a=\sqrt{\frac{1-w}{1-z}}, b=\frac{\varphi}{\sqrt{2(1-z)}}$, and with both $1-z$ and $\varphi$ arbitrarily small. Now Proposition 1.10 says that $\xi_{0}(w, z, \varphi) \asymp(1-z)^{-\frac{d}{2}}(1+b)^{-1}$, and the corollary follows from this combined with Proposition 6.2.

6.3. An exact formula for $\Phi_{\mathbf{0}}(\xi, \boldsymbol{w}, \boldsymbol{z})$ when $\xi(1-z)^{\frac{d-1}{2}}$ is large. We will now prove that if $\xi(1-z)^{\frac{d-1}{2}}$ is sufficiently large, then the formula (5.27), with range of integration $S$ in place of $S^{\prime}$, holds without error terms. We will use this result to complete the proof of Theorem 1.8, but it is clearly also of independent interest; for example we expect that when $d=3$ this result could be used to find completely explicit formulas for $\Phi_{0}$ in certain parameter regimes with $\xi$ large (we stress however that we anticipate any such explicit formula to be rather complicated). In view of this independent interest we allow a more general choice of $\boldsymbol{w}, \boldsymbol{z}$ than in (4.37) when stating the result; this does not cause any extra difficulties in the proof.

Proposition 6.5. There is a constant $c_{20}>0$ which only depends on $d$ such that for any $z, w, \varphi$ with $0 \leq z \leq w<1,0 \leq \varphi \leq \frac{\pi}{2}$, any $\xi \geq c_{20}(1-z)^{\frac{1-d}{2}}$, and any $\boldsymbol{w}, \boldsymbol{z} \in \mathcal{B}_{1}^{d-1}$ with $\|\boldsymbol{w}\|=w,\|\boldsymbol{z}\|=z, \varphi(\boldsymbol{w}, \boldsymbol{z})=\varphi$ and $\varphi\left(\boldsymbol{e}_{1}, \boldsymbol{z}\right) \leq \varphi, \varphi\left(\boldsymbol{e}_{1}, \boldsymbol{w}\right) \leq \varphi$, we have

$$
\Phi_{\mathbf{0}}(\xi, \boldsymbol{w}, \boldsymbol{z})=\zeta(d)^{-1} \int_{S} \Upsilon\left(\boldsymbol{z}, \boldsymbol{w}, \boldsymbol{v}^{\prime}, \xi^{-\frac{d-1}{d}} v_{1}(\boldsymbol{y} \cdot \boldsymbol{v})^{-1}\right) \frac{d \boldsymbol{v}}{(\boldsymbol{y} \cdot \boldsymbol{v})^{d}},
$$

where $\boldsymbol{y}=\xi^{\frac{1}{d}}(1, \boldsymbol{z}+\boldsymbol{w})$ and where $S$ is as in (5.9).

(To be more precise: $S=\left\{\boldsymbol{v} \in \mathrm{S}_{1}^{d-1}: 0<v_{1}<1, \boldsymbol{y} \cdot \boldsymbol{v}>c_{4} \xi^{\frac{1}{d}}\right\}$, where the constant $c_{4} \in\left(0, \frac{1}{2}\right)$ is as on p. 46,

The proof of Proposition 6.5 basically consists in going through the reductions carried out in Section 5.1, checking that at each step the error is in fact zero, provided that $\xi(1-z)^{\frac{d-1}{2}}$ is sufficiently large. We start by proving a couple of auxiliary lemmas.

Lemma 6.6. Assume $0<b_{1} \leq b_{2} \leq \ldots \leq b_{d}$, let $B$ be the ellipsoid

$$
B=\left\{\left(x_{1}, \ldots, x_{d}\right):\left(\frac{x_{1}}{b_{1}}\right)^{2}+\ldots+\left(\frac{x_{d}}{b_{d}}\right)^{2} \leq 1\right\}
$$

(i.e. ON half axes $b_{1}, \ldots, b_{d}$ ), and let $\Pi \subset \mathbb{R}^{d}$ be an arbitrary linear subspace of dimension $k$. Then $\operatorname{vol}_{k}(\Pi \cap B)$ is larger than or equal to the volume of a $k$-dimensional ellipsoid with (ON) half axes $b_{1}, b_{2}, \ldots, b_{k}$.

Proof. Indeed, a simple application of the min-max principle in linear algebra shows that $\Pi \cap B$ is an ellipsoid with ON half axes $0<b_{1}^{\prime} \leq \cdots \leq b_{k}^{\prime}$ satisfying $b_{j}^{\prime} \geq b_{j}, j=1, \ldots, k$. 
Lemma 6.7. For any $\boldsymbol{z} \in \mathcal{B}_{1}^{d-1}$ and $\xi>0$, if $M \in G$ satisfies $\mathbb{Z}^{d} M \cap \xi^{\frac{1}{d}}(\mathfrak{Z}(0,1,1)+(0, \boldsymbol{z}))=\emptyset$ and furthermore is generic in the sense that $\left(\mathbb{Z} \boldsymbol{e}_{3}+\cdots+\mathbb{Z} \boldsymbol{e}_{d}\right) M \cap \boldsymbol{e}_{1}^{\perp}=\{\mathbf{0}\}$, then we necessarily have $a_{1} a_{2} \ll \xi^{\frac{2-d}{d}}(1-z)^{\frac{1-d}{2}}$ in the Iwasawa decomposition of $M$ (cf. (3.3), (3.1)).

Proof. Let $\boldsymbol{z}, \xi, M$ satisfy the stated assumptions. After a rotation we may assume $\boldsymbol{z}=z \boldsymbol{e}_{1}$, $0 \leq z<1$. Set $\mathfrak{Z}=\xi^{\frac{1}{d}}(\mathfrak{Z}(0,1,1)+(0, \boldsymbol{z}))$. Note that $\mathbb{Z}^{d} M \cap \mathfrak{Z}=\emptyset$ implies $\mathbb{Z}^{d} M \cap(-\mathfrak{Z})=\emptyset$. Hence in view of the genericity assumption we have

$$
\left(\mathbb{Z} \boldsymbol{e}_{3}+\cdots+\mathbb{Z} \boldsymbol{e}_{d}\right) M \cap\left(\mathfrak{Z} \cup \boldsymbol{e}_{1}^{\perp} \cup(-\mathfrak{Z})\right)=\{\mathbf{0}\} .
$$

Now if $B$ denotes the ellipsoid

$$
B=\left\{\left(x_{1}, \ldots, x_{d}\right):\left(2 x_{1}\right)^{2}+\left(\frac{2 x_{2}}{1-z}\right)^{2}+\frac{4 d}{1-z}\left(x_{3}^{3}+\ldots+x_{d}^{2}\right) \leq 1\right\} .
$$

then

$$
\xi^{\frac{1}{d}} B \subset \mathfrak{Z} \cup e_{1}^{\perp} \cup(-\mathfrak{Z}) .
$$

Indeed, if $\left(x_{1}, \ldots, x_{d}\right) \in B$ then $\left|x_{1}\right| \leq \frac{1}{2},\left|x_{2}\right| \leq \frac{1}{2}(1-z)$ and $\left|x_{j}\right| \leq(4 d)^{-\frac{1}{2}}(1-z)^{\frac{1}{2}}$ for $j=3, \ldots, d$, and thus also

$$
\left\|\left(x_{2}, \ldots, x_{d}\right)-\boldsymbol{z}\right\|<\left(z+\frac{1}{2}(1-z)\right)^{2}+(d-2) \frac{1-z}{4 d}<\frac{1}{4}(1+z)^{2}+\frac{1}{4}(1-z)<1,
$$

which proves the claim.

It follows that

$$
\left(\mathbb{Z} \boldsymbol{e}_{3}+\cdots+\mathbb{Z} \boldsymbol{e}_{d}\right) M \cap \xi^{\frac{1}{d}} B=\{\mathbf{0}\} .
$$

However $\left(\mathbb{Z} \boldsymbol{e}_{3}+\cdots+\mathbb{Z} \boldsymbol{e}_{d}\right) M$ is a lattice of covolume $a_{3} \cdots a_{d}$ in the $(d-2)$-dimensional subspace $\left(\mathbb{R} \boldsymbol{e}_{3}+\cdots+\mathbb{R} \boldsymbol{e}_{d}\right) M \subset \mathbb{R}^{d}$ (cf. (3.3) $)$; furthermore $\left(\mathbb{R} \boldsymbol{e}_{3}+\cdots+\mathbb{R} \boldsymbol{e}_{d}\right) M \cap \xi^{\frac{1}{d}} B$ is an ellipsoid centered at $\mathbf{0}$ which by Lemma 6.6 has volume $\gg \xi^{\frac{d-2}{d}}(1-z)^{\frac{d-1}{2}}$. Hence by Minkowski's Theorem (cf., e.g., [26, Thm. 10]) we must have $a_{3} \cdots a_{d} \gg \xi^{\frac{d-2}{d}}(1-z)^{\frac{d-1}{2}}$. This proves the lemma, since $a_{1} a_{2}=\left(a_{3} \cdots a_{d}\right)^{-1}$.

Proof of Proposition 6.5. Recall equation (5.2) in Section 5.1, It was proved in [29, Prop. 7.3] that if $c_{20}$ is sufficiently large (which we assume from now on) then our assumption $\xi \geq$ $c_{20}(1-z)^{\frac{1-d}{2}}$ implies that all terms with $k_{1} \neq 1$ in (5.2) vanish. Hence

$$
\Phi_{\mathbf{0}}(\xi, \boldsymbol{w}, \boldsymbol{z})=\sum_{\boldsymbol{k}^{\prime} \in \mathbb{Z}^{d-1}} \nu_{\boldsymbol{y}}\left(\left\{M \in G_{\boldsymbol{k}, \boldsymbol{y}} \cap \mathcal{F}_{d}: \mathbb{Z}^{d} M \cap \mathfrak{Z}=\emptyset\right\}\right),
$$

where we write $\boldsymbol{k}=\left(1, \boldsymbol{k}^{\prime}\right)$.

We next use Lemma 3.4 with $A=c_{4} \xi^{\frac{1}{d}}$. (Note $\xi \geq c_{20}$, so that $A>1$ certainly holds provided $c_{20}$ is sufficiently large.) For any $\boldsymbol{k}=\left(1, \boldsymbol{k}^{\prime}\right)$, by Lemma 6.7 we have $a_{1} a_{2} \ll$ $\xi^{\frac{2-d}{d}}(1-z)^{\frac{1-d}{2}}$ for $\nu_{\boldsymbol{y}^{-a l m o s t}}$ all $M \in G_{\boldsymbol{k}, \boldsymbol{y}}$ with $\mathbb{Z}^{d} M \cap \mathfrak{Z}=\emptyset$; thus by taking $c_{20}$ sufficiently large we can force $a_{1} a_{2}<\left(c_{1}^{(d-1)}\right)^{-1} A^{2}$ to hold for these $M$, and it follows that the set $\mathcal{C}$ in (3.19) satisfies $\nu_{\boldsymbol{y}}\left(\left\{M \in G_{\boldsymbol{k}, \boldsymbol{y}} \cap \mathcal{C}: \mathbb{Z}^{d} M \cap \mathfrak{Z}=\emptyset\right\}\right)=0$. Hence, recalling (3.15) and the discussion between (5.6) and (5.8), we have

$$
\Phi_{\mathbf{0}}(\xi, \boldsymbol{w}, \boldsymbol{z})=\zeta(d)^{-1} \int_{S} \mu^{(d-1)}\left(\left\{\underset{\sim}{M} \in \mathcal{F}_{d-1}: L_{\boldsymbol{v}, M} \cap \mathfrak{Z}=\emptyset\right\}\right) \frac{d \boldsymbol{v}}{(\boldsymbol{y} \cdot \boldsymbol{v})^{d}} .
$$

Thus, in order to prove (6.8) it now suffices to prove that, with $a_{1}=\boldsymbol{y} \cdot \boldsymbol{v}$,

$$
\mu^{(d-1)}\left(\left\{\underset{\sim}{M} \in \mathcal{F}_{d-1}: L_{\boldsymbol{v}, M} \cap \mathfrak{Z}=\emptyset\right\}\right)=\Upsilon\left(\boldsymbol{z}, \boldsymbol{w}, \boldsymbol{v}^{\prime}, \xi^{-\frac{d-1}{d}} v_{1} a_{1}^{-1}\right)
$$

holds for all $\boldsymbol{v} \in S$.

Note that if $\boldsymbol{v}$ has the property that $a_{1}^{-\frac{1}{d-1}} \iota\left(\mathbb{Z}^{d-1} \underset{\sim}{\sim}\right) f(\boldsymbol{v}) \cap(\mathfrak{Z} \cup(\boldsymbol{y}-\mathfrak{Z})) \neq \emptyset$ for $\mu^{(d-1)}$-almost all $\underset{\sim}{M} \in \mathcal{F}_{d-1}$ then $\mu^{(d-1)}\left(\left\{\underset{\sim}{M} \in \mathcal{F}_{d-1}: L_{\boldsymbol{v}, M} \cap \mathfrak{Z}=\emptyset\right\}\right)=0$ and also $\Upsilon\left(\boldsymbol{z}, \boldsymbol{w}, \boldsymbol{v}^{\prime}, \xi^{-\frac{d-1}{d}} v_{1} a_{1}^{-1}\right)=$ 
0, cf. (5.20) and the argument we used to get (3.56). Hence from now on we may assume that $\boldsymbol{v}$ does not have the above property, i.e. we may assume that $\boldsymbol{v}$ satisfies

$$
\mu^{(d-1)}\left(\left\{\underset{\sim}{M} \in \mathcal{F}_{d-1}: \mathbb{Z}^{d-1} \underset{\sim}{\sim} \cap a_{1}^{\frac{1}{d-1}}\left(\mathfrak{Z}_{\boldsymbol{v}} \cup \mathfrak{Z}_{\boldsymbol{v}}^{\prime}\right)=\emptyset\right\}\right)>0,
$$

where $\mathfrak{Z} v=\iota^{-1}\left(\mathfrak{Z} f(\boldsymbol{v})^{-1}\right)$ and $\mathfrak{Z}_{\boldsymbol{v}}^{\prime}=\iota^{-1}\left((\boldsymbol{y}-\mathfrak{Z}) f(\boldsymbol{v})^{-1}\right)$.

We keep $\boldsymbol{v} \in S$, and as before we write $\omega_{\boldsymbol{w}}=\varphi\left(\boldsymbol{v}^{\prime}, \boldsymbol{w}\right)$ and $\omega_{\boldsymbol{z}}=\varphi\left(\boldsymbol{v}^{\prime}, \boldsymbol{z}\right)$. By [29, Lemma 7.1], $\mathfrak{Z} \boldsymbol{v}$ contains an open right $(d-1)$-dimensional cone $\mathfrak{C}_{\boldsymbol{z}}$ with $\mathbf{0}$ in its base, which has radius $r_{\boldsymbol{z}}$, height $h_{\boldsymbol{z}}$ and edge ratio $e_{\boldsymbol{z}}$, where

$$
r_{\boldsymbol{z}} \asymp \xi^{\frac{1}{d}}\left(1-z+\sin ^{2} \omega_{\boldsymbol{z}}\right)^{\frac{1}{2}}, \quad h_{\boldsymbol{z}} \asymp \xi^{\frac{1}{d}} \min \left(1, \frac{1-z+\omega_{\boldsymbol{z}}^{2}}{v_{1}}\right), \quad e_{\boldsymbol{z}} \asymp \min \left(1, \frac{1-z}{\sin ^{2} \omega_{\boldsymbol{z}}}\right),
$$

and, since $\boldsymbol{y}-\mathfrak{Z}=\xi^{\frac{1}{d}}(\mathfrak{Z}(0,1,1)+(0, \boldsymbol{w})), \mathfrak{Z}_{\boldsymbol{v}}^{\prime}$ contains an open right $(d-1)$-dimensional cone $\mathfrak{C}_{\boldsymbol{w}}$ with $\mathbf{0}$ in its base, which has radius $r_{\boldsymbol{w}}$ and height $h_{\boldsymbol{w}}$, where

$$
r_{\boldsymbol{w}} \asymp \xi^{\frac{1}{d}}\left(1-w+\sin ^{2} \omega_{\boldsymbol{w}}\right)^{\frac{1}{2}}, \quad h_{\boldsymbol{w}} \asymp \xi^{\frac{1}{d}} \min \left(1, \frac{1-w+\omega_{\boldsymbol{w}}^{2}}{v_{1}}\right) .
$$

Now by [29, Cor. 1.4] applied with $\mathfrak{C}_{\boldsymbol{z}}$, and using $a_{1} \gg \xi^{\frac{1}{d}}$, we see that (6.13) forces $e_{\boldsymbol{z}^{2}}^{\frac{d-1}{2}} \xi^{\frac{1}{d}} h_{\boldsymbol{z}} r_{\boldsymbol{z}}^{d-2} \ll 1$. If $v_{1}<1-z+\omega_{\boldsymbol{z}}^{2}$ then (6.14) would give

$$
1 \gg e^{\frac{d-1}{2}} \xi^{\frac{1}{d}} h_{\boldsymbol{z}} r_{\boldsymbol{z}}^{d-2} \gg \xi(1-z)^{\frac{d-1}{2}}\left(1-z+\sin ^{2} \omega_{\boldsymbol{z}}\right)^{-\frac{1}{2}} \gg \xi(1-z)^{\frac{d-1}{2}},
$$

which is impossible if $c_{20}$ is sufficiently large. Hence we must have $v_{1} \geq 1-z+\omega_{\boldsymbol{z}}^{2}$ (in particular $\left.\omega_{\boldsymbol{z}}<1\right)$. We now obtain

$$
1 \gg e^{\frac{d-1}{2}} \xi^{\frac{1}{d}} h_{\boldsymbol{z}} r_{\boldsymbol{z}}^{d-2} \gg \xi(1-z)^{\frac{d-1}{2}} \frac{\sqrt{1-z}+\omega_{\boldsymbol{z}}}{v_{1}} .
$$

Hence we conclude that, for any $\boldsymbol{v} \in S$ satisfying our assumption (6.13),

$$
\omega_{\boldsymbol{z}} \ll \xi^{-1}(1-z)^{\frac{1-d}{2}} v_{1} \quad \text { and } \quad(1-z)^{\frac{1}{2}} \ll \xi^{-1}(1-z)^{\frac{1-d}{2}} v_{1} .
$$

In particular by taking $c_{20}$ large we can force $\omega_{\boldsymbol{z}}$ to be less than any fixed small constant of our choice. Also since $\omega_{\boldsymbol{w}} \leq \varphi+\omega_{\boldsymbol{z}} \leq \frac{\pi}{2}+\omega_{\boldsymbol{z}}$ we may from now on assume $\omega_{\boldsymbol{w}}<\frac{3}{4} \pi$.

Note from the proof of [29, Lemma 7.1] that the heights of the cones $\mathfrak{C}_{\boldsymbol{z}}$ and $\mathfrak{C}_{\boldsymbol{w}}$ are both parallel to the line

$$
L=\iota^{-1}\left(\left(\boldsymbol{v}^{\perp} \cap \operatorname{Span}\left\{\boldsymbol{e}_{1}, \boldsymbol{v}\right\}\right) f(\boldsymbol{v})^{-1}\right)=\mathbb{R} \iota^{-1}\left(\left(\left\|\boldsymbol{v}^{\prime}\right\|^{2},-v_{1} \boldsymbol{v}^{\prime}\right) f(\boldsymbol{v})^{-1}\right) \subset \mathbb{R}^{d-1} .
$$

Thus if we let $T \in G^{(d-1)}$ be the linear map which acts by scalar multiplication by $\left(h_{\boldsymbol{w}} / r_{\boldsymbol{w}}\right)^{\frac{1}{d-1}}$ on every vector in $L^{\perp} \subset \mathbb{R}^{d-1}$ and multiplication by $\left(h_{\boldsymbol{w}} / r_{\boldsymbol{w}}\right)^{\frac{2-d}{d-1}}$ on every vector in $L$, then $a_{1}^{\frac{1}{d-1}} \mathfrak{C}_{\boldsymbol{w}} T$ is a cone which has both height and radius $=a_{1}^{\frac{1}{d-1}} h_{\boldsymbol{w}}^{\frac{1}{d-1}} r_{\boldsymbol{w}}^{\frac{d-2}{d-1}}$, and hence by Lemma3.1 any $\underset{\sim}{M} \in \mathcal{F}_{d-1} \subset \mathcal{S}_{d-1}$ with $\mathbb{Z}^{d-1} \underset{\sim}{\sim} \cap a_{1}^{\frac{1}{d-1}} C_{\boldsymbol{w}} T=\emptyset$ must have ${\underset{\sim}{a}}_{1} \gg a_{1}^{\frac{1}{d-1}} h_{\boldsymbol{w}}^{\frac{1}{d-1}} r_{\boldsymbol{w}}^{\frac{d-2}{d-1}}$. Also $a_{1}^{\frac{1}{d-1}} \mathfrak{C}_{\boldsymbol{z}} T$ is a cone with radius $r_{\boldsymbol{z}}^{\prime}$ and height $h_{\boldsymbol{z}}^{\prime}$, where

$$
r_{\boldsymbol{z}}^{\prime}=a_{1}^{\frac{1}{d-1}}\left(\frac{h_{\boldsymbol{w}}}{r_{\boldsymbol{w}}}\right)^{\frac{1}{d-1}} r_{\boldsymbol{z}}, \quad h_{\boldsymbol{z}}^{\prime}=a_{1}^{\frac{1}{d-1}}\left(\frac{h_{\boldsymbol{w}}}{r_{\boldsymbol{w}}}\right)^{\frac{2-d}{d-1}} h_{\boldsymbol{z}}
$$

and edge ratio $e_{\boldsymbol{z}}$ as before. Let us temporarily assume $\omega_{\boldsymbol{w}}^{2}>1-z+\omega_{\boldsymbol{z}}^{2}$. We then claim that $h_{\boldsymbol{z}}^{\prime} \ll r_{\boldsymbol{z}}^{\prime}$. Indeed, using (6.14) and (6.15), and recalling that $v_{1} \geq 1-z+\omega_{\boldsymbol{z}}^{2}$ and $\omega_{\boldsymbol{w}}<\frac{3}{4} \pi$, this claim is seen to be equivalent with

$$
\frac{\left(1-z+\omega_{\boldsymbol{z}}^{2}\right)^{\frac{1}{2}}\left(1-w+\omega_{\boldsymbol{w}}^{2}\right)^{\frac{1}{2}}}{v_{1}} \ll \min \left(1, \frac{1-w+\omega_{\boldsymbol{w}}^{2}}{v_{1}}\right),
$$


which is true because of $1-z+\omega_{\boldsymbol{z}}^{2}<1-w+\omega_{\boldsymbol{w}}^{2} \ll 1$ and (6.16). Now since $h_{\boldsymbol{z}}^{\prime} \ll r_{\boldsymbol{z}}^{\prime}$ we may just as well assume $h_{\boldsymbol{z}}^{\prime} \leq r_{\boldsymbol{z}}^{\prime}$, for if $h_{\boldsymbol{z}}^{\prime}>r_{\boldsymbol{z}}^{\prime}$ then we may shrink the cone $C_{\boldsymbol{z}}$ by keeping the base fixed while decreasing $h_{\boldsymbol{z}}$ until $h_{\boldsymbol{z}}^{\prime}=r_{\boldsymbol{z}}^{\prime}$, and (6.14) remains true. Now (6.13) implies

$$
\mu^{(d-1)}\left(\left\{\underset{\sim}{M} \in \mathcal{F}_{d-1}: \mathbb{Z}^{d-1} \underset{\sim}{\sim} \cap a_{1}^{\frac{1}{d-1}}\left(\mathfrak{C}_{\boldsymbol{z}} \cup \mathfrak{C}_{\boldsymbol{w}}\right) T=\emptyset\right\}\right)>0,
$$

and thus [29, Lemma 7.4] applies to give $e_{\boldsymbol{z}} \ll\left(\left(a_{1}^{\frac{1}{d-1}} h_{\boldsymbol{w}}^{\frac{1}{d-1}} r_{\boldsymbol{w}}^{\frac{d-2}{d-1}}\right) h_{\boldsymbol{z}^{\prime}}^{\prime} r^{\prime}{ }^{d-3}\right)^{-\frac{2}{d-1}}$. Hence using (6.17), 6.14), 6.15) we conclude

$$
\omega_{\boldsymbol{w}} \ll \xi^{-1}(1-z)^{\frac{1-d}{2}} v_{1} .
$$

This is of course true also when $\omega_{\boldsymbol{w}}^{2} \leq 1-z+\omega_{\boldsymbol{z}}^{2}$, by (6.16); hence (6.18) holds for all $\boldsymbol{v} \in S$ satisfying our assumption (6.13).

By (6.16) and (6.18) we can force both $\omega_{\boldsymbol{z}}$ and $\omega_{\boldsymbol{w}}$ to be less than any fixed small constant of our choice, by taking $c_{20}$ large. Hence also $\varphi$ and $\varphi\left(\boldsymbol{v}^{\prime}, \boldsymbol{e}_{1}\right)$ are forced to be small, since $\varphi \leq \omega_{\boldsymbol{z}}+\omega_{\boldsymbol{w}}$ and $\varphi\left(\boldsymbol{v}^{\prime}, \boldsymbol{e}_{1}\right) \leq \omega_{\boldsymbol{z}}+\varphi\left(\boldsymbol{z}, \boldsymbol{e}_{1}\right) \leq \omega_{\boldsymbol{z}}+\varphi$. Furthermore both $z$ and $w$ must be near 1 , by (6.16) and using $z \leq w<1$. Hence by Lemma 5.1, if $c_{20}$ is sufficiently large then $\left(n a_{1} \boldsymbol{v}+\boldsymbol{v}^{\perp}\right) \cap \mathfrak{Z}=\emptyset$ holds for all $\boldsymbol{v} \in S$ satisfying (6.13) and all $n \in \mathbb{Z} \backslash\{0,1\}$; and hence

$$
\begin{aligned}
\mu^{(d-1)}(\{\underset{\sim}{\mathcal{M}} & \left.\left.\in \mathcal{F}_{d-1}: L_{\boldsymbol{v}, M} \cap \mathfrak{Z}=\emptyset\right\}\right) \\
& =\mu^{(d-1)}\left(\left\{\underset{\sim}{\sim} \in \mathcal{F}_{d-1}: \mathbb{Z}^{d-1} \underset{\sim}{\sim} \cap a_{1}^{\frac{1}{d-1}}\left(\mathfrak{Z} \boldsymbol{v} \cup \mathfrak{Z}_{\boldsymbol{v}}^{\prime}\right)=\emptyset\right\}\right) .
\end{aligned}
$$

Furthermore by (6.16) we have $\frac{1-z+\omega_{z}^{2}}{v_{1}} \ll \frac{\sqrt{1-z}+\omega_{z}}{v_{1}} \ll \xi^{-1}(1-z)^{\frac{1-d}{2}}$, and hence by taking $c_{20}$ large we can force the ratio $\frac{1-z+\omega_{z}^{2}}{v_{1}}$ to be smaller than any fixed constant of our choice; similarly by (6.18) we can also force $\frac{1-w+\omega_{w}^{2}}{v_{1}}$ to be small. Hence by Lemma 3.10 (applied after appropriate rotations), if $c_{20}$ is sufficiently large then both $\boldsymbol{v}^{\perp} \cap \mathfrak{Z} \subset\left\{x_{1}<\frac{1}{2} \xi^{\frac{1}{d}}\right\}$ and $\boldsymbol{v}^{\perp} \cap(\boldsymbol{y}-\mathfrak{Z}) \subset\left\{x_{1}<\frac{1}{2} \xi^{\frac{1}{d}}\right\}$ must hold, for all $\boldsymbol{v} \in S$ satisfying (6.13). Hence by (6.19) and the same argument as we used to get (3.56), it follows that (6.12) holds for all such $\boldsymbol{v}$, and we are done.

6.4. Bound from above on the support of $\Phi_{\mathbf{0}}$. We will now prove an upper bound on $\xi_{0}(w, z, \varphi)$ which together with Proposition 6.2 will complete the proof of Theorem 1.8. We first prove a weak form of the desired statement.

Proposition 6.8. There is a constant $c_{21}>1$ which only depends on $d$ such that for any $0 \leq z \leq w<1,0 \leq \varphi \leq \pi$ and any

$$
\xi \geq \max \left\{c_{21}(1-z)^{\frac{1-d}{2}}, 2^{1-\frac{d}{2}}(1-z)^{-\frac{d}{2}} \rho\left(\sqrt{\frac{1-w}{1-z}}, \frac{\varphi}{\sqrt{2(1-z)}}\right)^{-1}\left(1+c_{21} \xi^{-2}(1-z)^{1-d}\right)\right\},
$$

we have $\Phi_{\mathbf{0}}(\xi, w, z, \varphi)=0$.

Proof. Assume $0 \leq z \leq w<1,0 \leq \varphi \leq \pi, \xi \geq c_{21}(1-z)^{\frac{1-d}{2}}$ (where we will successively impose conditions on $c_{21}$ being sufficiently large), and $\Phi_{0}(\xi, w, z, \varphi)>0$. Then by Proposition 1.10 we have $\left(1-z+\varphi^{2}\right)^{\frac{1}{2}} \ll \xi^{-1}(1-z)^{\frac{1-d}{2}}$. Hence if $c_{21}$ is sufficiently large then $1-c_{11} \leq z \leq w<1$ and $0 \leq \varphi \leq c_{11}$, where $c_{11}$ is the constant in Proposition 4.13. We also require $c_{21} \geq c_{20}$; then by Proposition 6.5 we have

$$
\Phi_{\mathbf{0}}(\xi, \boldsymbol{w}, \boldsymbol{z})=\zeta(d)^{-1} \int_{S} \Upsilon\left(\boldsymbol{z}, \boldsymbol{w}, \boldsymbol{v}^{\prime}, \xi^{-\frac{d-1}{d}} v_{1}(\boldsymbol{y} \cdot \boldsymbol{v})^{-1}\right) \frac{d \boldsymbol{v}}{(\boldsymbol{y} \cdot \boldsymbol{v})^{d}}
$$

where we now take $\boldsymbol{w}, \boldsymbol{z} \in \mathcal{B}_{1}^{d-1}$ as in (4.37).

We saw in the proof of Proposition 6.5 that $\Upsilon\left(\boldsymbol{z}, \boldsymbol{w}, \boldsymbol{v}^{\prime}, \xi^{-\frac{d-1}{d}} v_{1}(\boldsymbol{y} \cdot \boldsymbol{v})^{-1}\right)>0$ can only hold for $\boldsymbol{v} \in S$ with $\omega_{\boldsymbol{z}}, \omega_{\boldsymbol{w}} \ll \xi^{-1}(1-z)^{\frac{1-d}{2}}$, and then we must also have $\varphi<c_{22} \xi^{-1}(1-z)^{\frac{1-d}{2}}$ and $\varphi\left(\boldsymbol{v}^{\prime}, \boldsymbol{e}_{1}\right)<c_{22} \xi^{-1}(1-z)^{\frac{1-d}{2}}$ (where $c_{22}>0$ is some constant which only depends on $d$ ), 
since $\varphi \leq \omega_{\boldsymbol{z}}+\omega_{\boldsymbol{w}}$ and $\varphi\left(\boldsymbol{v}^{\prime}, \boldsymbol{e}_{1}\right) \leq \omega_{\boldsymbol{z}}+\varphi\left(\boldsymbol{z}, \boldsymbol{e}_{1}\right) \leq \omega_{\boldsymbol{z}}+\varphi$. Thus by requiring $c_{21}>c_{22} / c_{11}$ we force $\varphi\left(\boldsymbol{v}^{\prime}, \boldsymbol{e}_{1}\right)<c_{11}$ (and hence afortiori $\frac{\left\|\boldsymbol{v}^{\prime \prime}\right\|}{\left\|\boldsymbol{v}^{\prime}\right\|}=\sin \varphi\left(\boldsymbol{v}^{\prime}, \boldsymbol{e}_{1}\right)<c_{11}$ ) to hold for all $\boldsymbol{v} \in S$ with $\Upsilon\left(\boldsymbol{z}, \boldsymbol{w}, \boldsymbol{v}^{\prime}, \xi^{-\frac{d-1}{d}} v_{1}(\boldsymbol{y} \cdot \boldsymbol{v})^{-1}\right)>0$. It now follows from our assumption $\Phi_{\mathbf{0}}(\xi, \boldsymbol{w}, \boldsymbol{z})>0$ together with (6.20) and Proposition 4.13 that there exists some $\boldsymbol{v} \in S$ satisfying $\varphi\left(\boldsymbol{v}^{\prime}, \boldsymbol{e}_{1}\right)<$ $c_{22} \xi^{-1}(1-z)^{\frac{1-d}{2}}$ and

$$
2^{1-\frac{d}{2}}(1-z)^{-\frac{d}{2}} \xi^{-\frac{d-1}{d}} \frac{v_{1}}{\boldsymbol{y} \cdot \boldsymbol{v}}\left\{1+c_{12}\left(1-z+\varphi^{2}+\frac{\left\|\boldsymbol{v}^{\prime \prime}\right\|^{2}}{\left\|\boldsymbol{v}^{\prime}\right\|^{2}}\right)\right\}>\rho\left(\sqrt{\frac{1-w}{1-z}}, \frac{\varphi}{\sqrt{2(1-z)}}\right)
$$

Note that the computation in (5.28) applies, and since both $\left(1-z+\varphi^{2}\right)^{\frac{1}{2}}$ and $\left\|\boldsymbol{v}^{\prime \prime}\right\|$ are $\ll \xi^{-1}(1-z)^{\frac{1-d}{2}}$, and $v_{1}+2 v_{2} \gg 1$ (as follows from $v_{1}>0$ and $\varphi\left(\boldsymbol{v}^{\prime}, \boldsymbol{e}_{1}\right)<c_{11}$ ), we get

$$
\boldsymbol{y} \cdot \boldsymbol{v}=\xi^{\frac{1}{d}}\left(v_{1}+2 v_{2}\right)\left(1+O\left(\xi^{-2}(1-z)^{1-d}\right)\right) .
$$

We require that $c_{21}$ is so large that the big $O$-term in the last expression has absolute value $\leq \frac{1}{2}$; it then follows that the left hand side of (6.21) is $<2^{1-\frac{d}{2}}(1-z)^{-\frac{d}{2}} \xi^{-1}\left(1+O\left(\xi^{-2}(1-z)^{1-d}\right)\right)$, and thus

$$
\xi<2^{1-\frac{d}{2}}(1-z)^{-\frac{d}{2}} \rho\left(\sqrt{\frac{1-w}{1-z}}, \frac{\varphi}{\sqrt{2(1-z)}}\right)^{-1}\left(1+O\left(\xi^{-2}(1-z)^{1-d}\right)\right) .
$$

Hence the statement of the proposition follows, after increasing $c_{21}$ if necessary so as to be larger than or equal to the implied constant in the last big $O$-term.

Corollary 6.9. We have $\rho(a, b) \asymp 1+|b|$, uniformly over all $0<a \leq 1, b \in \mathbb{R}$.

Proof. Because of Corollary 6.4 and $\rho(a,-b)=\rho(a, b)$, it suffices to prove $\rho(a, b) \ll 1+b$ for all $0<a \leq 1, b \geq 0$. Given any $a \in(0,1]$ and $b \geq 0$ we may find $z \leq w<1$ and $\varphi \geq 0$ satisfying $a=\sqrt{\frac{1-w}{1-z}}, b=\frac{\varphi}{\sqrt{2(1-z)}}$, and with both $1-z$ and $\varphi$ arbitrarily small. Now Proposition 1.10 says that $\xi_{0}(w, z, \varphi) \asymp(1-z)^{-\frac{d}{2}}(1+b)^{-1}$; thus if we take $1-z$ sufficiently small (for our fixed $a, b)$ we have $\xi_{0}(w, z, \varphi)>c_{21}(1-z)^{\frac{1-d}{2}}$. But $\Phi_{0}(\xi, w, z, \varphi)>0$ for all $\xi<\xi_{0}(w, z, \varphi)$, and hence Proposition 6.8 implies that $\xi_{0}(w, z, \varphi) \leq 2^{1-\frac{d}{2}}(1-z)^{-\frac{d}{2}} \rho(a, b)^{-1}\left(1+c_{21}^{-1}\right)$. Using here $\xi_{0}(w, z, \varphi) \asymp(1-z)^{-\frac{d}{2}}(1+b)^{-1}$ we obtain $\rho(a, b) \ll 1+b$, as desired.

Using Corollary 6.9 we are now able to make Proposition 6.8 a bit more precise, as follows:

Proposition 6.10. We have

$$
\xi_{0}(w, z, \varphi) \leq 2^{1-\frac{d}{2}}(1-z)^{-\frac{d}{2}} \rho\left(\sqrt{\frac{1-w}{1-z}}, \frac{\varphi}{\sqrt{2(1-z)}}\right)^{-1}\left\{1+O\left(\max (1-z, 1-w)+\varphi^{2}\right)\right\},
$$

uniformly over all $z, w \in[0,1), \varphi \in[0, \pi]$.

Proof. Because of $\xi_{0}(w, z, \varphi)=\xi_{0}(z, w, \varphi)$ and (6.1), we may assume $z \leq w$ without loss of generality. Let us write $a=\sqrt{\frac{1-w}{1-z}}$ and $b=\frac{\varphi}{\sqrt{2(1-z)}}$, as usual. It follows from Corollary 6.9 that there is a constant $0<c<1$ which only depends on $d$ such that $2^{1-\frac{d}{2}}(1-z)^{-\frac{d}{2}} \rho(a, b)^{-1}>$ $c_{21}(1-z)^{\frac{1-d}{2}}$ holds whenever $1-z \leq c$ and $0 \leq \varphi \leq c$. Hence for any such $z, w, \varphi$, if we let $\xi_{1}$ be the unique real positive solution to the equation

$$
\xi_{1}=2^{1-\frac{d}{2}}(1-z)^{-\frac{d}{2}} \rho(a, b)^{-1}\left(1+c_{21} \xi_{1}^{-2}(1-z)^{1-d}\right),
$$

then $\xi_{1}>c_{21}(1-z)^{\frac{1-d}{2}}$ and hence by Proposition 6.8 we have $\xi_{0}(w, z, \varphi) \leq \xi_{1}$. Also $\xi_{1} \asymp$ $(1-z)^{-\frac{d}{2}}\left(1+\frac{\varphi}{\sqrt{1-z}}\right)^{-1}$; thus $\xi_{1}^{-2}(1-z)^{1-d} \asymp 1-z+\varphi^{2}$ and

$$
\xi_{1}=2^{1-\frac{d}{2}}(1-z)^{-\frac{d}{2}} \rho(a, b)^{-1}\left(1+O\left(1-z+\varphi^{2}\right)\right),
$$


which implies the desired bound. In the remaining case when $1-z>c$ or $\varphi>c$, the desired bound follows automatically from Corollary 6.9 and Proposition 1.10 .

Note that Theorem 1.8 follows from Proposition 6.10 and Proposition 6.2, together with the discussion at the end of Section 6.1.

We may now also give the simple proof of Corollary 1.12:

Proof of Corollary 1.12. The existence and continuity of $\xi_{0}(w)$ is proved by a similar argument as for $\xi_{0}(w, z, \varphi)$ (cf. the end of Section 6.1), working directly from the definition of $\Phi(\xi, \boldsymbol{w})$, (2.2). In particular, the upper semicontinuity of $\xi_{0}(w)$ follows from the fact that $\Phi(\xi, w) \geq$ $\Phi\left(\xi^{\prime}, w^{\prime}\right)$ holds whenever $\xi^{\prime} \geq k^{d-1} \xi$, where $k=\frac{1+\varepsilon w}{1+\varepsilon w^{\prime}}$ with $\varepsilon=\operatorname{sgn}\left(w-w^{\prime}\right)$. This in turn follows from the inclusion $w \boldsymbol{e}_{2}+\mathfrak{Z}(0, \xi, 1) \subset\left(w^{\prime} \boldsymbol{e}_{2}+\mathfrak{Z}(0, \xi, 1)\right) D$, where $D=\operatorname{diag}(1, k, k, \ldots, k)$.

Next, in order to prove the asymptotic formula (1.42) we note that, by (1.14),

$$
\xi_{0}(w)=\sup \left\{\xi_{0}(w, z, \varphi): z \in[0,1), \varphi \in[0, \pi]\right\}, \quad \forall w \in[0,1) .
$$

It follows from Proposition 1.10 that there exists a constant $c>1$ which only depends on $d$ such that, for any $w \in[0,1)$, the supremum in (6.22) remains unchanged if we restrict to the set

$$
S_{w}:=\{(z, \varphi) \in[0,1) \times[0, \pi]: 1-z \leq c(1-w), \varphi \leq c \sqrt{1-w}\} .
$$

Hence by Theorem 1.8, as $w \rightarrow 1^{-}$we have

$$
\xi_{0}(w)=\left(\sup _{(z, \varphi) \in S_{w}} 2^{1-\frac{d}{2}}(1-w)^{-\frac{d}{2}} \rho\left(\sqrt{\frac{1-z}{1-w}}, \frac{\varphi}{\sqrt{2(1-w)}}\right)^{-1}\right)\{1+O(1-w)\} .
$$

However it is immediate from the definition (4.2) that $\Xi(\mathbf{0}, \boldsymbol{y} ; \boldsymbol{h} ; v) \leq \Xi(\mathbf{0}, \mathbf{0} ; \boldsymbol{h} ; v)$; hence $\rho(a, b) \geq \rho(1,0)$ for all $a>0, b \in \mathbb{R}$ (cf. (4.8) and (4.12) ), so that the supremum in (6.23) is attained at $z=w, \varphi=0$. Hence (1.42) holds.

6.5. Proof of Proposition 1.15. Changing to a slightly different topic, we now give the quick proof of Proposition 1.15 .

It follows e.g. from (1.22) and [29, Lemma 7.11] that $\Phi_{\mathbf{0}}(\xi)$ is a continuous and decreasing function of $\xi$; furthermore (1.14) and (1.22) imply $\Phi(\xi, \mathbf{0})=\int_{\xi}^{\infty} \Phi_{\mathbf{0}}(\eta) d \eta$. Hence we have $\Phi_{0}(\xi)>0$ if and only if $0<\xi<\xi_{0}(0)$, and by (2.2) this holds if and only if

$$
\mu\left(\left\{M \in X_{1}: \mathbb{Z}^{d} M \cap \mathfrak{Z}(0, \xi, 1)=\emptyset\right\}\right)>0 .
$$

Using $-\mathbb{Z}^{d} M=\mathbb{Z}^{d} M$ and the fact that $\mathbb{Z}^{d} M \cap \boldsymbol{e}_{1}^{\perp}=\{\mathbf{0}\}$ holds for $\mu$-almost all $M \in X_{1}$, we see that (6.24) holds if and only if

$$
\mu\left(\left\{M \in X_{1}: \mathbb{Z}^{d} M \cap \mathfrak{Z}(-\xi, \xi, 1)=\{\mathbf{0}\}\right\}\right)>0 .
$$

But note that $M \in X_{1}$ satisfies $\mathbb{Z}^{d} M \cap \mathfrak{Z}(-\xi, \xi, 1)=\{\mathbf{0}\}$ if and only if $\mathbb{Z}^{d} M$ is a packing lattice of $\frac{1}{2} \mathfrak{Z}(-\xi, \xi, 1)$ (cf., e.g., [17, Sec. 20, Thm 1]). Hence (6.25) holds if and only if $\operatorname{vol}\left(\frac{1}{2} \mathfrak{Z}(-\xi, \xi, 1)\right)<\delta_{d}^{*}(\mathfrak{Z})$, i.e. if and only if $2^{1-d} v_{d-1} \xi<\delta_{d}^{*}(\mathfrak{Z})$. Hence $\xi_{0}(0)=2^{d-1} v_{d-1}^{-1} \delta_{d}^{*}(\mathfrak{Z})$.

\section{Asymptotics For $\frac{\partial}{\partial \xi} \Phi(\xi, \boldsymbol{w})$ DeRIVEd From TheOrem 1.7}

In this section we use Theorem 1.7 and (1.14) to derive an asymptotic formula for $\frac{\partial}{\partial \xi} \Phi(\xi, \boldsymbol{w})$ as $\xi \rightarrow \infty$. In particular this results in a new proof of Theorem 1.11 (except for a slightly worse log-factor), and it also gives an internal check of consistency of our asymptotic formulas. 
Theorem 7.1. For any $d \geq 3$ we have

$$
\begin{aligned}
-\frac{\partial}{\partial \xi} \Phi(\xi, w)=\xi^{-3+\frac{2}{d}} G_{d}\left(\xi^{\frac{2}{d}}(1-w)\right) & \\
& +O\left(\xi^{-3} \log \left(2+\min \left(\xi, \xi^{-\frac{2}{d}}(1-w)^{-1}\right)\right)\left\{\begin{array}{ll}
\log \xi & \text { if } d=3 \\
1 & \text { if } d \geq 4
\end{array}\right\}\right),
\end{aligned}
$$

as $\xi \rightarrow \infty$, uniformly over all $0 \leq w<1$, where

$$
\begin{aligned}
& G_{d}(t)=\left(2-\frac{2}{d}\right) F_{d}(t)-\frac{2}{d} F_{d}^{\prime}(t) t \\
& =\frac{2^{3\left(1-\frac{d}{2}\right)} \pi^{\frac{d}{2}-1} t^{\frac{d}{2}-1}}{\Gamma\left(\frac{d}{2}-1\right) \zeta(d)} \int_{0}^{1} \int_{0}^{\infty} \Xi\left(\sigma, 2^{1-\frac{d}{2}} t^{-\frac{d}{2}} y\right) \sigma^{d-3} y(1-y)^{d-2} d \sigma d y .
\end{aligned}
$$

The function $G_{d}(t)$ is a bounded continuous function from $\mathbb{R}_{>0}$ to $\mathbb{R}_{\geq 0}$.

Note that the second equality in (7.2) follows immediately from the definition of $F_{d}(t)$ (cf. (3.75) ) and the fact that

$$
\begin{aligned}
\frac{d}{d t} \int_{0}^{1} \int_{0}^{\infty} \Xi\left(\sigma, 2^{1-\frac{d}{2}}\right. & \left.t^{-\frac{d}{2}} y\right) \sigma^{d-3}(1-y)^{d-1} d \sigma d y \\
& =\frac{d}{2} t^{-1} \int_{0}^{1} \int_{0}^{\infty} \Xi\left(\sigma, 2^{1-\frac{d}{2}} t^{-\frac{d}{2}} y\right) \sigma^{d-3}(1-d y)(1-y)^{d-2} d \sigma d y
\end{aligned}
$$

for all $t>0$. This last identity is proved by substituting $y=t^{\frac{d}{2}} x$ in the outer integral in the left hand side, then carrying out the differentiation with respect to $t$, and finally substituting back $x=t^{-\frac{d}{2}} y$. The fact that $G_{d}(t)$ is bounded and continuous is proved by the same argument as below (3.75), and similar considerations also justify the differentiation in (7.3). Note in particular that it follows that $F_{d}(t)$ is $\mathrm{C}^{1}$ on all $\mathbb{R}_{>0}$.

Note also that the main term in (7.1) is what is obtained by differentiation of the main term in (1.40). Hence Theorem 7.1 indeed implies Theorem 1.11 (except for a slightly worse $\log$-factor) upon integrating over $\xi \in\left[\xi_{0}, \infty\right)$, and using the fact that for any fixed $w$ we have $\Phi(\xi, w)=0$ for all sufficiently large $\xi$.

The key fact needed for the deduction of Theorem 7.1 from Theorem 1.7 is the following:

Lemma 7.2. For any $\boldsymbol{h} \in \mathbb{R}_{+}^{d-1}$ and $v>0$ we have (writing $\boldsymbol{h}^{\prime}=\left(h_{2}, \ldots, h_{d-1}\right)$ as usual)

$$
\int_{P^{d-1}} \Xi(\mathbf{0}, \boldsymbol{y} ; \boldsymbol{h} ; v) d \boldsymbol{y}=v \Xi\left(\frac{\left\|\boldsymbol{h}^{\prime}\right\|}{h_{1}}, v\right) .
$$

Proof. By definition we have

$\Xi(\mathbf{0}, \boldsymbol{y} ; \boldsymbol{h} ; v)=\int_{X_{1}^{(d-1)}} I\left(\left(v^{\frac{1}{d-1}} \mathbb{Z}^{d-1} M\right) \cap P_{\boldsymbol{h}}^{d-1}(\mathbf{0})=\emptyset\right) I\left(\left(v^{\frac{1}{d-1}} \mathbb{Z}^{d-1} M\right) \cap P_{\boldsymbol{h}}^{d-1}(\boldsymbol{y})=\emptyset\right) d \mu(M)$.

Hence by Fubini's Theorem the left hand side of (7.4) equals

$\int_{X_{1}} I\left(\left(v^{\frac{1}{d-1}} \mathbb{Z}^{d-1} M\right) \cap P_{\boldsymbol{h}}^{d-1}(\mathbf{0})=\emptyset\right) \operatorname{vol}_{\mathbb{R}^{d-1}}\left(\left\{\boldsymbol{y} \in P^{d-1}:\left(v^{\frac{1}{d-1}} \mathbb{Z}^{d-1} M\right) \cap P_{\boldsymbol{h}}^{d-1}(\boldsymbol{y})=\emptyset\right\}\right) d \mu(M)$.

But for $\mu$-almost every $M \in X_{1}$ we have $\mathbb{Z}^{d-1} M \cap \boldsymbol{h}^{\perp}=\{\mathbf{0}\}$, and for each such $M$ the set

$$
\left\{\boldsymbol{y} \in P^{d-1}:\left(v^{\frac{1}{d-1}} \mathbb{Z}^{d-1} M\right) \cap P_{\boldsymbol{h}}^{d-1}(\boldsymbol{y})=\emptyset\right\}
$$

is in fact a fundamental domain for $\mathbb{R}^{d-1} /\left(v^{\frac{1}{d-1}} \mathbb{Z}^{d-1} M\right)$. Indeed, for every $\boldsymbol{x} \in \mathbb{R}^{d-1}$ the set $\Omega_{\boldsymbol{x}}=P^{d-1} \cap\left(\boldsymbol{x}+v^{\frac{1}{d-1}} \mathbb{Z}^{d-1} M\right)$ is infinite, and there is exactly one $\boldsymbol{y} \in \Omega_{\boldsymbol{x}}$ for which $\left(v^{\frac{1}{d-1}} \mathbb{Z}^{d-1} M\right) \cap P_{\boldsymbol{h}}^{d-1}(\boldsymbol{y})=\emptyset$, namely that $\boldsymbol{y} \in \Omega_{\boldsymbol{x}}$ for which $\boldsymbol{y} \cdot \boldsymbol{h}$ is minimal (the uniqueness is guaranteed since $\mathbb{Z}^{d-1} M \cap \boldsymbol{h}^{\perp}=\{\mathbf{0}\}$ ). Hence the volume of the set in (7.5) equals $v$, and the lemma follows. 
Proof of Theorem 7.1. As in section 3.4 we fix the constant $c_{3}$ so that $c_{3} \geq \sqrt{\sigma_{d}(1,0)}$ (cf. (4.14)) and $\Phi(\xi, w)=0$ whenever $1-w \geq c_{3} \xi^{-\frac{2}{d}}$. Then $G_{d}(t)=0$ for all $t \geq c_{3}$, and hence (7.1) is automatic whenever $1-w \geq c_{3} \xi^{-\frac{2}{d}}$. Hence from now on we will assume $1-w<c_{3} \xi^{-\frac{2}{d}}$.

By (1.14) we have:

$$
-\frac{\partial}{\partial \xi} \Phi(\xi, w)=\int_{\mathcal{B}_{1}^{d-1}} \Phi_{\mathbf{0}}\left(\xi, w \boldsymbol{e}_{1}, \boldsymbol{z}\right) d \boldsymbol{z} .
$$

Let us write $\varphi:=\varphi\left(\boldsymbol{z}, \boldsymbol{e}_{1}\right)$ and $z=\|\boldsymbol{z}\|$. By Proposition 1.10 there is a constant $c>0$ which only depends on $d$ such that $\Phi_{\mathbf{0}}\left(\xi, w \boldsymbol{e}_{1}, \boldsymbol{z}\right)=0$ holds for all $\boldsymbol{z} \in \mathcal{B}_{1}^{d-1} \backslash\left(U_{1} \cup U_{2}\right)$, where

$$
\begin{aligned}
& U_{1}:=\left\{z \in \mathcal{B}_{1}^{d-1}: \varphi \leq \frac{\pi}{2} ; \varphi<c \xi^{-1}(1-\min (w, z))^{\frac{1-d}{2}} ; 1-\min (z, w)<c \xi^{-\frac{2}{d}}\right\} ; \\
& U_{2}:=\left\{\boldsymbol{z} \in \mathcal{B}_{1}^{d-1}: \varphi>\frac{\pi}{2} ; 1-\min (z, w)<c\left(\xi^{-\frac{2}{d-2}}+(\xi /(\pi-\varphi))^{-\frac{2}{d-1}}\right)\right\} .
\end{aligned}
$$

Now for $\boldsymbol{z} \in U_{1}$ we apply Theorem 1.7 to $\Phi_{\mathbf{0}}\left(\xi, w \boldsymbol{e}_{1}, \boldsymbol{z}\right)=\Phi_{\mathbf{0}}(\xi, w, z, \varphi)=\Phi_{\mathbf{0}}(\xi, z, w, \varphi)$, while for $\boldsymbol{z} \in U_{2}$ we apply the bound from Theorem [1.9. This gives

$$
\begin{aligned}
&-\frac{\partial}{\partial \xi} \Phi(\xi, w)=\int_{U_{1}}\left(\frac { 2 ^ { 2 - \frac { 3 } { 2 } d } ( 1 - w ) ^ { \frac { d } { 2 } - 1 } \xi ^ { - 1 } } { \zeta ( d ) } \int _ { ( 0 , 1 ) \times \mathbb { R } ^ { d - 2 } } \Xi \left(\sqrt{\frac{1-z}{1-w}}, \frac{\varphi}{\sqrt{2(1-w)}} ; \boldsymbol{h} ;\right.\right. \\
&\left.\left.2^{1-\frac{d}{2}}(1-w)^{-\frac{d}{2}} \xi^{-1}\left(1-h_{1}\right)\right) d \boldsymbol{h}+O(E)\right) d \boldsymbol{z} \\
&+O\left(\int_{U_{2}} \xi^{-2} \min \left\{1,\left(\xi(\pi-\varphi)^{d-2}\right)^{-1+\frac{2}{d-1}}\right\} d \boldsymbol{z}\right),
\end{aligned}
$$

where $E$ is as in (1.30).

We parametrize $\boldsymbol{z} \in \mathcal{B}_{1}^{d-1}$ as

$$
(0,1) \times(0, \pi) \times \mathrm{S}_{1}^{d-3} \ni\langle z, \varphi, \boldsymbol{\omega}\rangle \mapsto \boldsymbol{z}=(z \cos \varphi, z(\sin \varphi) \boldsymbol{\omega}) \in \mathcal{B}_{1}^{d-1} .
$$

Then

$$
d \boldsymbol{z}=z^{d-2}(\sin \varphi)^{d-3} d z d \varphi d \boldsymbol{\omega} .
$$

Let us first consider the contribution from the error term $O(E)$ in (7.7). For $z \in U_{1}$ we have $0 \leq \varphi<\min \left(\frac{\pi}{2}, c \xi^{-1}(1-w)^{\frac{1-d}{2}}\right)$ and $0<1-z<\min \left(c \xi^{-\frac{2}{d}},\left(c^{-1} \xi \varphi\right)^{-\frac{2}{d-1}}\right) \ll$ $\xi^{-\frac{2}{d}} \min \left(1,\left(\xi \varphi^{d}\right)^{-\frac{2}{d(d-1)}}\right)$. Hence the contribution from the $O(E)$-term in (17.7) is:

$$
\ll \int_{0}^{\min \left(\frac{\pi}{2}, c \xi^{-1}(1-w)^{\frac{1-d}{2}}\right)} \xi^{-\frac{2}{d}} \min \left(1,\left(\xi \varphi^{d}\right)^{-\frac{2}{d(d-1)}}\right) E \varphi^{d-3} d \varphi
$$

We may assume that $c$ is so large that $c^{\frac{2}{d-1}}>c_{3}$. Then $\xi^{-\frac{1}{d}}<c \xi^{-1}(1-w)^{\frac{1-d}{2}}$, since $1-w<c_{3} \xi^{-\frac{2}{d}}$, and hence if $d \geq 4$ then we get (cf. (5.56))

$$
=\int_{0}^{\xi^{-1 / d}} \xi^{-2-\frac{2}{d}} \varphi^{d-3} d \varphi+\int_{\xi^{-1 / d}}^{\min \left(\frac{\pi}{2}, c \xi^{-1}(1-w)^{\frac{1-d}{2}}\right)} \xi^{-3} \varphi^{-1} d \varphi \ll \xi^{-3} \log \left(2+\min \left(\xi, \xi^{-\frac{2}{d}}(1-w)^{-1}\right)\right) .
$$

When $d=3$ we get the same bound except for an extra factor $\log \xi$. The integral over $U_{2}$ in (7.7) is easily seen to be $\ll \xi^{-3}$. (This bound was also pointed out in [29, Cor. 1.10].) Hence we have

$$
\begin{aligned}
& -\frac{\partial}{\partial \xi} \Phi(\xi, w)=\frac{2^{2-\frac{3}{2} d}}{\zeta(d)}(1-w)^{\frac{d}{2}-1} \xi^{-1} \int_{U_{1}} \int_{(0,1) \times \mathbb{R}^{d-2}} \Xi\left(\sqrt{\frac{1-z}{1-w}}, \frac{\varphi}{\sqrt{2(1-w)}} ; \boldsymbol{h} ;\right. \\
& \left.2^{1-\frac{d}{2}}(1-w)^{-\frac{d}{2}} \xi^{-1}\left(1-h_{1}\right)\right) d \boldsymbol{h} d \boldsymbol{z}+O\left(E^{\prime}\right),
\end{aligned}
$$

where we write $E^{\prime}$ for the error majorant in the second line of (17.1). 
Next note that in (7.8) we have

$$
\left|z^{d-2}(\sin \varphi)^{d-3}-\varphi^{d-3}\right| \ll\left(1-z+\varphi^{2}\right) \varphi^{d-3} \ll\left(\xi^{-\frac{2}{d}}+\varphi^{2}\right) \varphi^{d-3},
$$

uniformly over all $\boldsymbol{z} \in U_{1}$. Using this fact together with Lemma 5.3 we see that the error caused by replacing $d \boldsymbol{z}$ by $\varphi^{d-3} d z d \varphi d \boldsymbol{\omega}$ in (7.9) is

$$
\ll \int_{U_{1}} \min \left(\xi^{-2+\frac{2}{d}}, \xi^{-3+\frac{2}{d-1}} \varphi^{-d+\frac{2}{d-1}}\right)\left(\xi^{-\frac{2}{d}}+\varphi^{2}\right) \varphi^{d-3} d z d \varphi d \boldsymbol{\omega},
$$

and this is seen to be $\ll E^{\prime}$ by a computation which is very similar to the computation above bounding the contribution from $O(E)$ in (7.7). Hence

$$
\begin{array}{r}
-\frac{\partial}{\partial \xi} \Phi(\xi, w)=\frac{2^{2-\frac{3}{2} d}}{\zeta(d)}(1-w)^{\frac{d}{2}-1} \xi^{-1} \int_{U_{1}} \int_{(0,1) \times \mathbb{R}^{d-2}} \Xi\left(\sqrt{\frac{1-z}{1-w}}, \frac{\varphi}{\sqrt{2(1-w)}} ; \boldsymbol{h} ;\right. \\
\left.2^{1-\frac{d}{2}}(1-w)^{-\frac{d}{2}} \xi^{-1}\left(1-h_{1}\right)\right) d \boldsymbol{h} \varphi^{d-3} d z d \varphi d \boldsymbol{\omega}+O\left(E^{\prime}\right) .
\end{array}
$$

Now by Corollary 6.4 and (6.1) we see that by requiring that the constant $c$ has been chosen sufficiently large (in a way that only depends on $d$ ) we have that any tuple $\left\langle z^{\prime}, w^{\prime}, \varphi, \xi\right\rangle \in\left(\mathbb{R}_{>0}\right)^{4}$ for which

$$
\int_{(0,1) \times \mathbb{R}^{d-2}} \Xi\left(\sqrt{\frac{z^{\prime}}{w^{\prime}}}, \frac{\varphi}{\sqrt{2 w^{\prime}}} ; \boldsymbol{h} ; 2^{1-\frac{d}{2}} w^{\prime-\frac{d}{2}} \xi^{-1}\left(1-h_{1}\right)\right) d \boldsymbol{h}>0
$$

must satisfy both $\varphi<c \xi^{-1} \max \left(z^{\prime}, w^{\prime}\right)^{\frac{1-d}{2}}$ and $\max \left(z^{\prime}, w^{\prime}\right)<c \xi^{-\frac{2}{d}}$. From now on we assume that $\xi$ is so large that $c \xi^{-\frac{2}{d}}<1$. It then follows that in the outer integral in (17.10) we may extend the range $U_{1}$ to the larger set of all

$$
\langle z, \varphi, \boldsymbol{\omega}\rangle \in(-\infty, 1) \times\left(0, \min \left(\frac{\pi}{2}, c \xi^{-1}(1-w)^{\frac{1-d}{2}}\right)\right) \times \mathrm{S}_{1}^{d-3}
$$

without changing the value of the integral. In fact if $c \xi^{-1}(1-w)^{\frac{1-d}{2}} \leq \frac{\pi}{2}$ then we may extend the range all the way to $(-\infty, 1) \times \mathbb{R}_{>0} \times \mathrm{S}_{1}^{d-3}$ without changing the value of the integral; on the other hand if $c \xi^{-1}(1-w)^{\frac{1-d}{2}}>\frac{\pi}{2}$ then we may extend the range to $(-\infty, 1) \times \mathbb{R}_{>0} \times \mathrm{S}_{1}^{d-3}$ at the cost of an error which is

$$
\begin{aligned}
\ll \int_{\frac{\pi}{2}}^{\infty} \min \left(\xi^{-2+\frac{2}{d}}, \xi^{-3+\frac{2}{d-1}} \varphi^{-d+\frac{2}{d-1}}\right) \cdot \xi^{-\frac{2}{d}} \min \left(1,\left(\xi \varphi^{d}\right)^{-\frac{2}{d(d-1)}}\right) \varphi^{d-3} d \varphi \ll \xi^{-3} & \int_{\frac{\pi}{2}}^{\infty} \varphi^{-3} d \varphi \\
& \ll \xi^{-3} \ll E^{\prime} .
\end{aligned}
$$

Hence we obtain, after substituting $\varphi=\sqrt{2(1-w)} r$ and using (4.8) (note that the following is correct also for $d=3$, with the convention that $\operatorname{vol}_{\mathrm{S}_{1}^{0}}\left(\mathrm{~S}_{1}^{0}\right)=2$ ):

$$
\begin{array}{r}
-\frac{\partial}{\partial \xi} \Phi(\xi, w)=\frac{2^{1-d}{ }^{{ }^{2} \mathrm{~S}_{1}^{d-3}\left(\mathrm{~S}_{1}^{d-3}\right)}}{\zeta(d)}(1-w)^{d-2} \xi^{-1} \int_{-\infty}^{1} \int_{0}^{\infty} \int_{(0,1) \times \mathbb{R}^{d-2}} \Xi(\mathbf{0}, \\
\left.\left(\frac{1-z}{1-w}+r^{2}-1\right) \boldsymbol{e}_{1}+r \boldsymbol{e}_{2} ; \boldsymbol{h} ; 2^{1-\frac{d}{2}}(1-w)^{-\frac{d}{2}} \xi^{-1}\left(1-h_{1}\right)\right) d \boldsymbol{h} r^{d-3} d r d z \\
+O\left(E^{\prime}\right) .
\end{array}
$$

Next, using (4.6) we see that $\int_{(0,1) \times \mathbb{R}^{d-2}} \Xi\left(\mathbf{0}, x_{1} \boldsymbol{e}_{1}+x_{2} \boldsymbol{e}_{2} ; \boldsymbol{h} ; v\right) d \boldsymbol{h}=\int_{(0,1) \times \mathbb{R}^{d-2}} \Xi\left(\mathbf{0}, x_{1} \boldsymbol{e}_{1}+\right.$ $\left.x_{2} \boldsymbol{\omega} ; \boldsymbol{h} ; v\right) d \boldsymbol{h}$ for any $\boldsymbol{\omega} \in \mathrm{S}_{1}^{d-3}$ and any $x_{1}, x_{2} \in \mathbb{R}$ with $x_{1}>x_{2}^{2}-1$. Integrating over all $\boldsymbol{\omega} \in \mathrm{S}_{1}^{d-3}$ we get that the main term in (7.12) equals

$$
\begin{array}{r}
\frac{2^{1-d}}{\zeta(d)}(1-w)^{d-2} \xi^{-1} \int_{-\infty}^{1} \int_{\boldsymbol{e}_{1}^{\perp}} \int_{(0,1) \times \mathbb{R}^{d-2}} \Xi\left(\mathbf{0},\left(\frac{1-z}{1-w}+\|\boldsymbol{u}\|^{2}-1\right) \boldsymbol{e}_{1}+\boldsymbol{u} ; \boldsymbol{h} ;\right. \\
\left.2^{1-\frac{d}{2}}(1-w)^{-\frac{d}{2}} \xi^{-1}\left(1-h_{1}\right)\right) d \boldsymbol{h} d \boldsymbol{u} d z
\end{array}
$$


where $\boldsymbol{e}_{1}^{\perp}$ is the orthogonal complement of $\boldsymbol{e}_{1}$ in $\mathbb{R}^{d-1}$. Substituting now $z=1-(1-w) y_{1}$ and then letting $\boldsymbol{y}:=y_{1} \boldsymbol{e}_{1}+\boldsymbol{u}$, we get

$$
=\frac{2^{1-d}}{\zeta(d)}(1-w)^{d-1} \xi^{-1} \int_{P^{d-1}} \int_{(0,1) \times \mathbb{R}^{d-2}} \Xi\left(\mathbf{0}, \boldsymbol{y} ; \boldsymbol{h} ; 2^{1-\frac{d}{2}}(1-w)^{-\frac{d}{2}} \xi^{-1}\left(1-h_{1}\right)\right) d \boldsymbol{h} d \boldsymbol{y} .
$$

Applying now Lemma 7.2 and Fubini's Theorem we get

$$
\begin{aligned}
& =\frac{2^{2-\frac{3}{2} d}}{\zeta(d)}(1-w)^{\frac{d}{2}-1} \xi^{-2} \int_{(0,1) \times \mathbb{R}^{d-2}}\left(1-h_{1}\right) \Xi\left(\frac{\left\|\boldsymbol{h}^{\prime}\right\|}{h_{1}}, 2^{1-\frac{d}{2}}(1-w)^{-\frac{d}{2}} \xi^{-1}\left(1-h_{1}\right)\right) d \boldsymbol{h} \\
& =\frac{2^{3\left(1-\frac{d}{2}\right)} \pi^{\frac{d}{2}-1}}{\Gamma\left(\frac{d}{2}-1\right) \zeta(d)}(1-w)^{\frac{d}{2}-1} \xi^{-2} \int_{0}^{1} \int_{0}^{\infty} \Xi\left(\sigma, 2^{1-\frac{d}{2}}(1-w)^{-\frac{d}{2}} \xi^{-1} y\right) \sigma^{d-3} y(1-y)^{d-2} d \sigma d y
\end{aligned}
$$

where we substituted $\boldsymbol{h}=(1-y)(1, \sigma \boldsymbol{\omega})\left(0<y<1, \sigma>0, \boldsymbol{\omega} \in \mathrm{S}_{1}^{d-3}\right)$ and used the fact that $\operatorname{vol}\left(\mathrm{S}_{1}^{d-3}\right)=\frac{2 \pi^{\frac{d}{2}-1}}{\Gamma\left(\frac{d}{2}-1\right)}$. Hence (7.1) is proved.

\section{INDEX OF NOTATIONS}

$\left[a_{1}, \boldsymbol{v}, \boldsymbol{u}, M\right]$ the $\mathrm{SL}(d, \mathbb{R})$-matrix defined by (3.9), (3.10)

$[\boldsymbol{p}, \boldsymbol{x}]_{\boldsymbol{y}} \sim$ the $3 \times 3$ matrix defined by (2.14), (2.15)

a $(a)$ the diagonal matrix in (3.1) 19

$\mathcal{B}_{r}^{d} \quad$ open ball in $\mathbb{R}^{d}$ of radius $r$, centered at the origin 2

$\mathfrak{C}_{\boldsymbol{h}}(w) \quad$ the cut ball in (3.54) 28

$\mathfrak{C}_{\boldsymbol{h}}(\boldsymbol{w})$ the cut ball in 44.34)

$\mathfrak{C}_{h}(\boldsymbol{z}, \boldsymbol{w}) \quad=\mathfrak{C}_{h}(\boldsymbol{w}) \cup \mathfrak{C}_{h}(\boldsymbol{z})$

$\boldsymbol{e}_{1} \quad=(1,0, \ldots, 0)$

$\boldsymbol{e}_{2} \quad=(0,1,0, \ldots, 0)$

$\mathcal{F}_{d} \quad$ a fundamental domain for $\Gamma^{(d)} \backslash G^{(d)}$

$F(t) \quad=\pi-\arccos (t)+t \sqrt{1-t^{2}}$

$F_{d}(t) \quad$ the function in (3.75) (for $d \geq 3$ ) 32

$F_{\mathbf{0}, d}\left(t_{1}, t_{2}, \alpha\right)$ the function in (5.66) (for $d \geq 3$ )

$G, G^{(d)} \quad=\operatorname{SL}(d, \mathbb{R})$

$\mathcal{G}_{A} \quad$ the subset of $\mathrm{SL}(d, \mathbb{R})$ in 3.15

$H \quad=\left\{g \in \mathrm{SL}(d, \mathbb{R}): \boldsymbol{e}_{1} g=\boldsymbol{e}_{1}\right\}$

$M_{\alpha, \beta} \quad$ the linear map in (4.7) 34

$\mathrm{n}(u)$ the upper triangular matrix in (3.2)

$P^{d-1} \quad=\left\{\left(x_{1}, \ldots, x_{d-1}\right) \in \mathbb{R}^{d-1}: x_{1}>x_{2}^{2}+\ldots+x_{d-1}^{2}-1\right\}$

$P_{h}^{d-1} \quad$ the cut paraboloid $P^{d-1} \cap \mathbb{R}_{h-}^{d-1}$

$P_{\boldsymbol{h}}^{d-1}(\boldsymbol{y}) \quad$ the cut paraboloid in (4.1)

$P_{u, r} \quad$ the paraboloid in (3.59) 29

$\boldsymbol{q}_{\boldsymbol{y}_{\boldsymbol{d}} \boldsymbol{p}, \boldsymbol{x}}(\boldsymbol{x}) \quad$ the $\mathbb{R}^{3}$-vector in 2.15)

$\mathbb{R}_{+}^{d-1} \quad=\left\{\left(h_{1}, \ldots, h_{d-1}\right) \in \mathbb{R}^{d-1}: h_{1}>0\right\}$

$\mathbb{R}_{\boldsymbol{h - 1}}^{d-1} \quad\left\{\boldsymbol{x} \in \mathbb{R}^{d-1}: \boldsymbol{x} \cdot \boldsymbol{h}<0\right\}$

$\mathcal{S}_{d} \quad$ the Siegel set in (3.6)

$\mathcal{S}_{d}^{\prime} \quad$ the subset of $\mathcal{S}_{d}$ defined in (3.18)

$\mathrm{S}_{1}^{d-1} \quad$ unit sphere in $\mathbb{R}^{d}$

$\mathrm{S}_{1}^{\prime d-1} \quad$ the hemisphere $\left\{\left(v_{1}, \ldots, v_{d}\right) \in \mathrm{S}_{1}^{d-1}: v_{1}>0\right\}$

$S \quad$ the set defined in (5.9) 47

$S^{\prime} \quad$ the set defined in (3.42) (in section 3) or (5.12) (in section 5) 26, 48

$S^{\prime \prime} \quad$ the subset of $(0,1) \times \mathbb{R}^{d-2}$ defined just below (5.37)

$S^{\prime \prime \prime} \quad$ the subset of $(0,1) \times \mathbb{R}^{d-2}$ defined just below (5.40)

$T_{\alpha, \beta} \quad$ the affine linear map in (4.7) 34

$v_{d-1} \quad$ volume of the unit ball in $\mathbb{R}^{d-1}$

$X_{1}, X_{1}^{(d)}=\mathrm{SL}(d, \mathbb{Z}) \backslash \mathrm{SL}(d, \mathbb{R})$, space of lattices

$X_{1}(\boldsymbol{y}) \quad=\left\{M \in X_{1}: \boldsymbol{y} \in \mathbb{Z}^{d} M\right\}$, a submanifold of $X_{1}$

$X_{1}(\boldsymbol{k}, \boldsymbol{y}) \quad=\left\{\Gamma M \in X_{1}: M \in G, \boldsymbol{k} M=\boldsymbol{y}\right\}$

$\mathfrak{Z}\left(c_{1}, c_{2}, r\right)$ the cylinder in (2.1)

$\mathfrak{Z} v \quad=\iota^{-1}\left(\mathfrak{Z} f(\boldsymbol{v})^{-1}\right) \subset \mathbb{R}^{d-1}$ (for various sets $\mathfrak{Z} \subset \mathbb{R}^{d}$ )

$\delta_{d}^{*}(\mathfrak{Z}) \quad$ the maximal lattice packing density of a cylinder in $\mathbb{R}^{d}$ 


\begin{tabular}{|c|c|}
\hline$\Gamma, \Gamma^{(d)}$ & $=\mathrm{SL}(d, \mathbb{Z})$ \\
\hline$\iota$ & the embedding $\mathbb{R}^{d-1} \ni\left(x_{1}, \ldots, x_{d-1}\right) \mapsto\left(0, x_{1}, \ldots, x_{d-1}\right) \in \mathbb{R}^{d}$ \\
\hline$\mu, \mu^{(d)}$ & Haar measure on $G$, probability measure on $X_{1}$ \\
\hline$\nu_{y}$ & a probability measure on $X_{1}(\boldsymbol{y})$ (cf. [20, Sec. 7], [29, Sec. 5]) \\
\hline$\xi_{0}(w)$ & the function defined in Corollary 1.12 \\
\hline$\xi_{0}(w, z, \varphi)$ & the function defined in Theorem 1.8 \\
\hline$\xi_{1}(\boldsymbol{w}, \boldsymbol{z})$ & the function $\mathcal{B}_{1}^{2} \times \mathcal{B}_{1}^{2} \rightarrow \mathbb{R}$ defined just before Theorem 1.4 \\
\hline$\Xi(\sigma, v)$ & the lattice probability in 3.65 \\
\hline$\Xi\left(\boldsymbol{y}, \boldsymbol{y}^{\prime} ; \boldsymbol{h} ; v\right)$ & the lattice probability in (4.2) \\
\hline$\Xi(\boldsymbol{y} ; \boldsymbol{h} ; v)$ & $=\Xi(\boldsymbol{y}, \boldsymbol{y} ; \boldsymbol{h} ; v)$ \\
\hline$\Xi(a, b ; \boldsymbol{h} ; v)$ & $=\Xi\left(\mathbf{0},\left(a^{2}+b^{2}-1\right) \boldsymbol{e}_{1}+b \boldsymbol{e}_{2} ; \boldsymbol{h} ; v\right)$ \\
\hline$\rho(a, b)$ & $=\inf \left\{v>0: \exists \boldsymbol{h} \in \mathbb{R}_{+}^{d-1}: \Xi(a, b ; \boldsymbol{h} ; v)>0\right\}$ \\
\hline$\sigma_{d}(r, \alpha)$ & the function in (6.3) \\
\hline$\Upsilon(\boldsymbol{z}, \boldsymbol{w}, \boldsymbol{h}, v)$ & $=\mu\left(\left\{M \in X_{1}: \mathbb{Z}^{d-1} M \cap v^{-\frac{1}{d-1}} \mathfrak{C}_{\boldsymbol{h}}(\boldsymbol{z}, \boldsymbol{w})=\emptyset\right\}\right)$ \\
\hline$\Phi(\xi)$ & limiting distribution for the free path length \\
\hline $\bar{\Phi}_{\mathbf{0}}(\xi)$ & limiting distribution for the free path length \\
\hline$\Phi_{0}(\xi)$ & limiting distribution for the free path length \\
\hline$\Phi(\xi, \boldsymbol{w})$ & the collision kernel function defined in (2.2) \\
\hline$\Phi(\xi, w)$ & $=\Phi(\xi, \boldsymbol{w})$ with $w=\|\boldsymbol{w}\|$ \\
\hline$\Phi_{\mathbf{0}}(\xi, \boldsymbol{w}, \boldsymbol{z})$ & the collision kernel function defined in (2.4) \\
\hline$\Phi_{0}(\xi, w, z, \varphi)$ & $=\Phi_{\mathbf{0}}(\xi, \boldsymbol{w}, \boldsymbol{z})$ with $w=\|\boldsymbol{w}\|, z=\|\boldsymbol{z}\|$ and $\varphi=\varphi(\boldsymbol{w}, \boldsymbol{z})$ \\
\hline
\end{tabular}

\section{REFERENCES}

[1] J. S. Athreya and G. A. Margulis, Logarithm laws for unipotent flows, I, J. Mod. Dyn. 3 (2009), 359-378.

[2] F.P. Boca, R.N. Gologan and A. Zaharescu, The statistics of the trajectory of a certain billiard in a flat two-torus. Comm. Math. Phys. 240 (2003), 53-73.

[3] F.P. Boca and A. Zaharescu, The distribution of the free path lengths in the periodic two-dimensional Lorentz gas in the small-scatterer limit, Commun. Math. Phys. 269 (2007), 425-471.

[4] C. Boldrighini, L.A. Bunimovich and Y.G. Sinai, On the Boltzmann equation for the Lorentz gas. J. Statist. Phys. 32 (1983), 477-501.

[5] A. Borel, Introduction aux groupes arithmétiques, Hermann, Paris, 1969.

[6] J. Bourgain, F. Golse and B. Wennberg, On the distribution of free path lengths for the periodic Lorentz gas. Comm. Math. Phys. 190 (1998), 491-508.

[7] V.A. Bykovskii and A.V. Ustinov, Trajectory statistics in inhomogeneous Sinai problem for 2-dimensional lattice, Izv. Ran. Ser. Mat. 73 (2009), 17-36

[8] E. Caglioti and F. Golse, The Boltzmann-Grad limit of the periodic Lorentz gas in two space dimensions, C. R. Math. Acad. Sci. Paris 346 (2008) 477-482.

[9] P. Chiu, Covering with Hecke points, J. Number Theory 53 (1995) 25-44.

[10] L. Clozel, H. Oh, E. Ullmo, Hecke operators and equidistribution of Hecke points, Invent. Math. 144 (2001), 327-351.

[11] J. H. Conway and N. J. A. Sloane, Sphere Packings, Lattices and Groups, third edition, Springer-Verlag, New York, 1999.

[12] P. Dahlqvist, The Lyapunov exponent in the Sinai billiard in the small scatterer limit. Nonlinearity 10 (1997), 159-173.

[13] W. Duke, Z. Rudnick, P. Sarnak, Density of Integer Points on Affine Homogeneous Varieties, Duke Math. J. 71 (1993), 143-179.

[14] A. Friedman, Foundations of Modern Analysis, Dover Publications, Inc., New York, 1982.

[15] G. Gallavotti, Divergences and approach to equilibrium in the Lorentz and the Wind-tree-models, Physical Review 185 (1969), 308-322.

[16] F. Golse and B. Wennberg, On the distribution of free path lengths for the periodic Lorentz gas. II. M2AN Math. Model. Numer. Anal. 34 (2000), no. 6, 1151-1163.

[17] P. M. Gruber and C. G. Lekkerkerker, Geometry of numbers, North-Holland Publishing Co., Amsterdam, 1987.

[18] H. Lorentz, Le mouvement des électrons dans les métaux, Arch. Néerl. 10 (1905), 336-371.

[19] J. Marklof, Kinetic transport in crystals, Proceedings of the ICMP, Prague 2009, 162-179, World Scientic, 2010.

[20] J. Marklof and A. Strömbergsson, The distribution of free path lengths in the periodic Lorentz gas and related lattice point problems, arXiv:0706.4395 to appear in the Annals of Mathematics. 
[21] J. Marklof and A. Strömbergsson, The Boltzmann-Grad limit of the periodic Lorentz gas, arXiv:0801.0612 to appear in the Annals of Mathematics.

[22] J. Marklof and A. Strömbergsson, Kinetic transport in the two-dimensional periodic Lorentz gas, Nonlinearity 21 (2008) 1413-1422.

[23] C. A. Rogers, Packing and covering, Cambridge University Press, New York, 1964.

[24] P. Sarnak, Diophantine problems and linear groups, Proceedings of the International Congress of Mathematics 1 (1991), Springer Tokyo, pp. 459-471.

[25] W. M. Schmidt, Masstheorie in der Geometrie der Zahlen, Acta Math. 102 (1959), 159-224.

[26] C. L. Siegel, Lectures on the Geometry of Numbers, Springer-Verlag, Berlin-Heidelberg-New York, 1989.

[27] H. Spohn, The Lorentz process converges to a random flight process, Comm. Math. Phys. 60 (1978), $277-290$.

[28] A. Strömbergsson, A. Venkatesh, Small solutions to linear congruences and Hecke equidistribution, Acta Arith., 118 (2005), 41-78.

[29] A. Strömbergsson, On the probability of a random lattice avoiding a large convex set, arXiv:1008.3805.

[30] C. Zong, On the packing densities and the covering densities of the Cartesian products of convex bodies, Monatsh. Math., 145 (2005), 73-81.

[31] A. C. Woods, The critical determinant of a spherical cylinder, J. London Math. Soc., 33 (1958), 357-368

School of Mathematics, University of Bristol, Bristol BS8 1TW, U.K.

j.marklof@bristol.ac.uk

Department of Mathematics, Box 480, Uppsala University, SE-75106 Uppsala, Sweden

astrombe@math.uu.se 